\title{
Functional analysis of STRIPAK complex components in the filamentous ascomycete Sordaria macrospora
}

\author{
Dissertation \\ for the award of the degree \\ "Doctor rerum naturalium" \\ of the Georg-August-Universität Göttingen
}

within the doctoral program Microbiology and Biochemistry

of the Georg-August University School of Science (GAUSS)

submitted by

Eva Johanna Reschka

from Tarnowitz (Poland)

Göttingen, 2017 

Members of the Thesis Committee

Prof. Stefanie Pöggeler

Department of Genetics of Eukaryotic Microorganisms, Institute of Microbiology and Genetics

Prof. Gerhard Braus

Department of Molecular Microbiology and Genetics, Institute of Microbiology and Genetics

Prof. Henning Urlaub

Bioanalytical Mass Spectrometry Group, Max Planck Institute for Biophysical Chemistry

Members of the Examination Board

$1^{\text {st }}$ Referee Prof. Stefanie Pöggeler

Department of Genetics of Eukaryotic Microorganisms, Institute of Microbiology and Genetics

$2^{\text {nd }}$ Referee Prof. Kai Heimel

Department of Molecular Microbiology and Genetics, Institute of Microbiology and Genetics

\section{Further members of the Examination Board}

Prof. Gerhard Braus

Department of Molecular Microbiology and Genetics, Institute of Microbiology and Genetics

\section{Prof. Henning Urlaub}

Bioanalytical Mass Spectrometry Group, Max Planck Institute for Biophysical Chemistry

\section{Prof. Rolf Daniel}

Department of Genomic and Applied Microbiology, Institute of Microbiology and Genetics

\section{Prof. Heike Krebber}

Department of Molecular Genetics, Institute of Microbiology and Genetics

Date of oral examination: 18.10.2017 
Affirmation

I hereby declare that this thesis was written independently and with no other sources and aids than quoted.

Göttingen, 25.08.2017

Eva Johanna Reschka 
This doctoral study was performed in the group of Prof. Stefanie Pöggeler in the Department of Genetics of Eukaryotic Microorganisms at the Institute of Microbiology and Genetics, GeorgAugust University Göttingen. 



\section{Table of Contents}

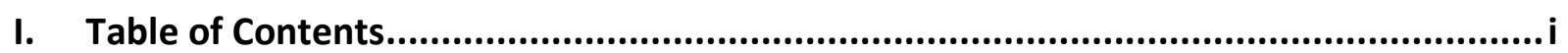

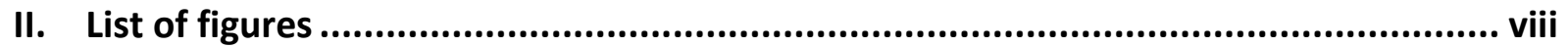

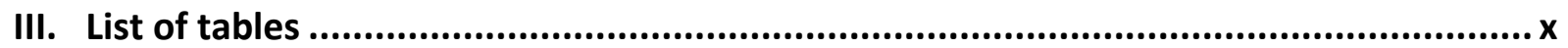

IV. List of figures in the Supplement ...................................................................

V. List of videos in the Supplement/DVD ............................................................ xii

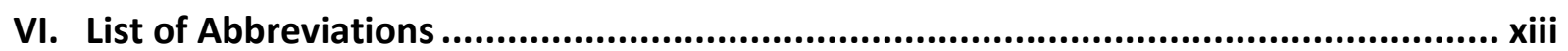

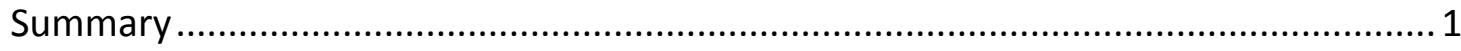

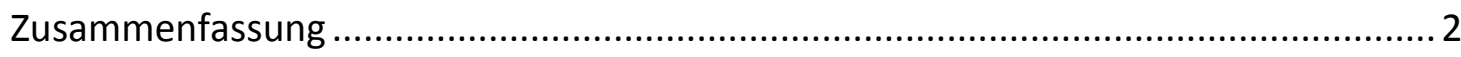

1. Introduction ..................................................................................................... 4

1.1 Components of mammalian STRIPAK complex ................................................6

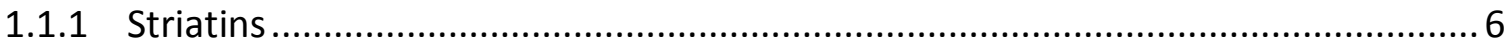

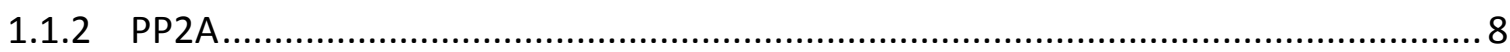

1.1 .3 STRIP1/2

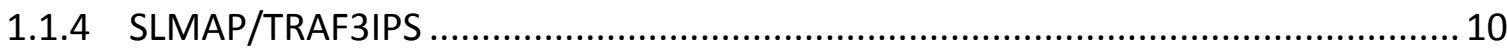

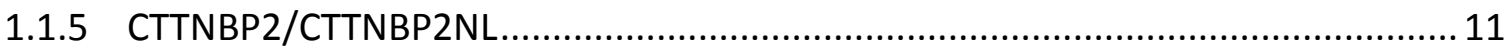

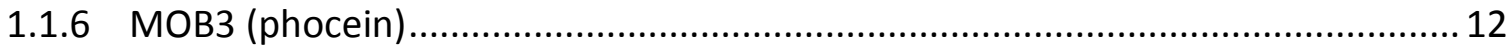

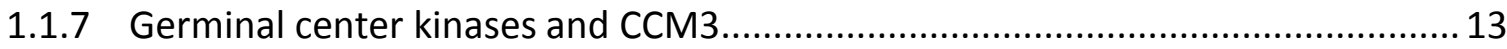

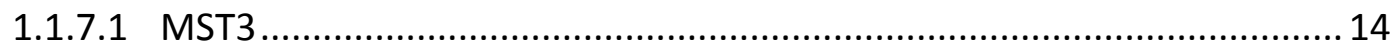

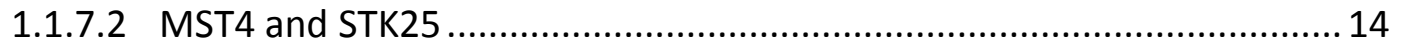

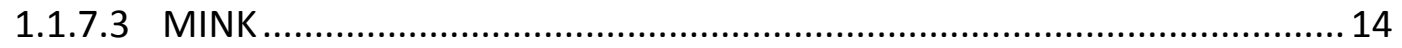

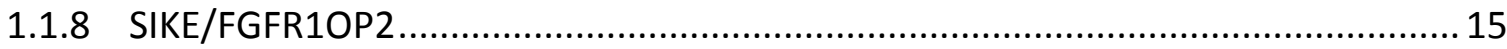




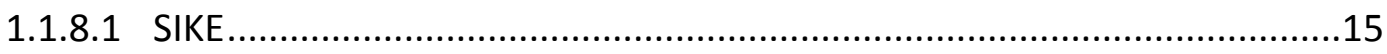

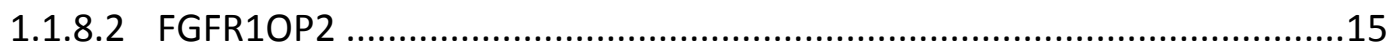

1.2 STRIPAK-like complexes in yeasts and in Drosophila melanogaster ......................... 16

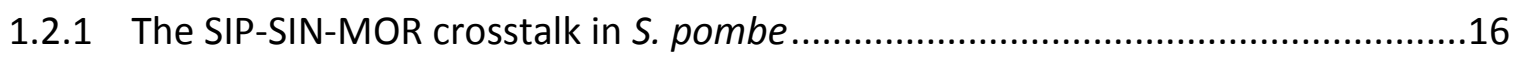

1.2.2 The Far complex in Saccharomyces cerevisiae ...............................................18

1.2.2.1 The Far complex in S. cerevisiae is involved in pheromone-mediated cell cycle arrest

1.2.2.2 The Far complex regulates cell growth, autophagy and actin polarization

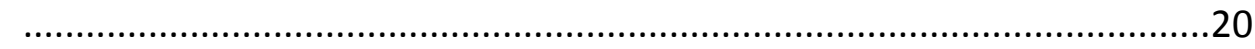

1.2.2.3 MEN regulates mitotic exit and cytokinesis in S. cerevisiae ..................21

1.2.3 STRIPAK complex in Drosophila melanogaster ...............................................22

1.2.3.1 Hippo signaling in D. melanogaster ...............................................22

1.2.3.2 Cka in the Jun-N-terminal kinase pathway in D. melanogaster ..............24

1.2.3.3 The dSTRIPAK activates the Ras-Raf-MAPK/ERK pathway ......................25

1.3 Sordaria macrospora is a model organism to study fruiting-body development......... 26

1.3.1 The STRIPAK complex in S. macrospora regulates sexual development and hyphal fusion

1.3.2 Striatin orthologs in filamentous fungi control hyphal fusion, sexual development and vegetative growth

1.3.3 Fungal MOB3 proteins are involved in hyphal fusion, development and vesicular traffic .29

1.3.4 The fungal STRIP1/2 ortholog and the catalytic PP2ac1 subunit link the fungal STRIPAK to septation and the CWI pathway. 30

1.3.5 The SLMAP ortholog PRO45 is part of the fungal STRIPAK complex in S. macrospora

1.3.6 Fungal STRIPAK-associated proteins SmGPI1 and GCKIII kinases SmKIN3 and SmKIN24

1.4 Aims of this thesis. 


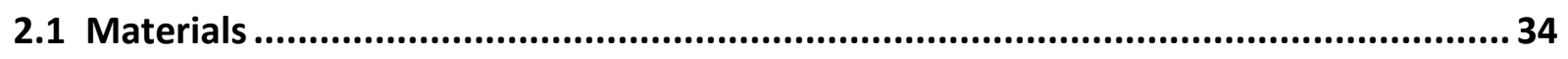

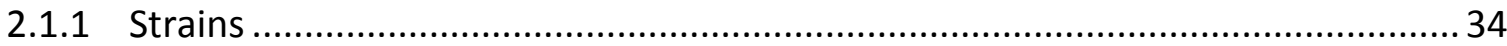

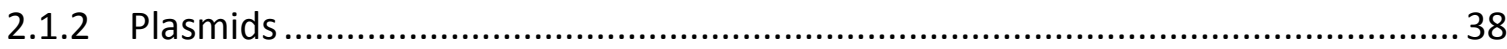

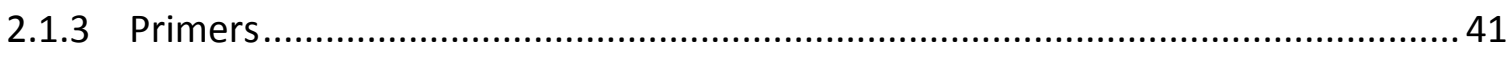

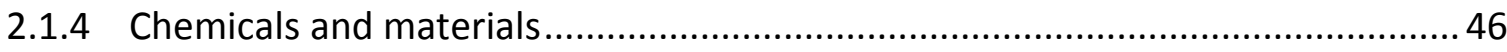

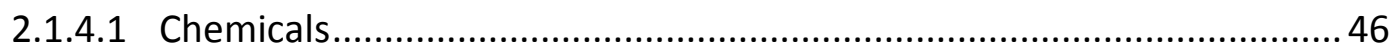

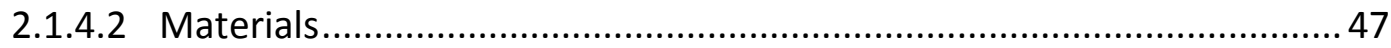

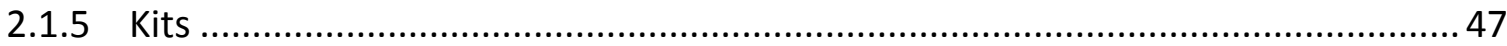

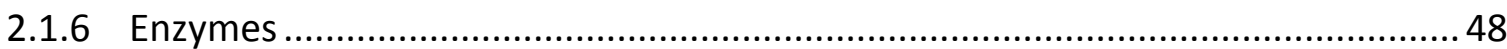

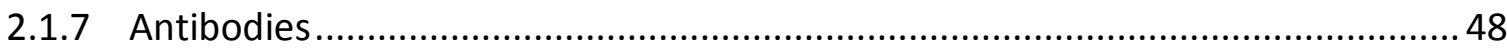

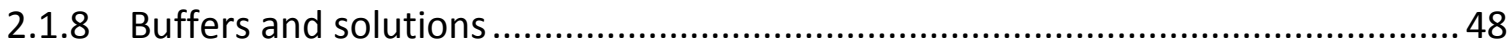

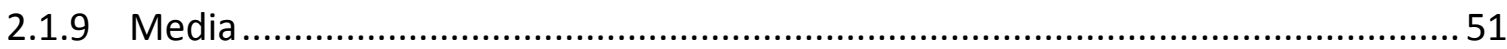

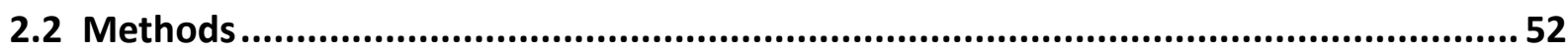

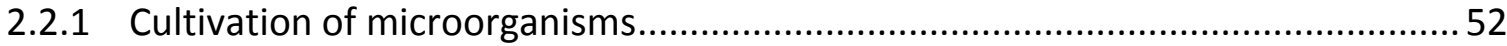

2.2.2 Preparation of competent microorganisms and transformation procedure ........53

2.2.3 Generation of S. macrospora deletion strains............................................... 54

2.2.3.1 Generation of S. macrospora single deletion strains ........................... 54

2.2.3.2 Generation of S. macrospora double deletion strains .......................... 55

2.2.4 Generation of sci1 expression plasmids for transformation into S. macrospora .56

2.2.4.1 Generation of sci1 full length constructs for transformation into $S$.

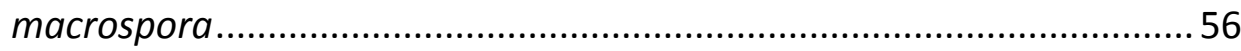

2.2.4.2 Construction of plasmids containing fragments of sci1 and transformation into S. macrospora ............................................................................ 56

2.2.5 Transformation of wt S. macrospora strains with pHA_nat and pFLAG_nat........57

2.2.6 Generation of expression plasmids for complementation attempts of $S$. macrospora with the mammalian FGFR1OP2 and yeast Far3 and Far7 .............. 58

2.2.7 Generation of S. macrospora strains expressing fluorescent fusion proteins ...... 58 
2.2.7.1 Complementation of the $\Delta \operatorname{sci}_{\text {hyg }} \Delta$ pro11 strain ..................................58

2.2.7.2 Generation of S. macrospora strains for analysis of nuclei, mitochondria, $E R$, actin, nuclear envelope as well as early and late endosomes with fluorescence microscopy

2.2.7.3 Generation of SmPOM152 expression plasmid for transformation into S. macrospora 59

2.2.7.4 Generation of $\Delta$ pro11::hapro11egfp strain .....................................59

2.2.8 Molecular biology methods: nucleic acids 59

2.2.8.1 PCR 59

2.2.8.2 Separation of nucleic acids with agarose-gel electrophoresis .60

2.2.8.3 Purification of nucleic acids .60

2.2.8.4 Hydrolysis and ligation of nucleic acids .61

2.2.8.5 Isolation of plasmid DNA from E. coli 61

2.2.8.6 Isolation of plasmid DNA from $S$. cerevisiae. 61

2.2.8.7 Isolation of RNA and genomic DNA from S. macrospora .61

2.2.8.8 Southern blotting and hybridization ..............................................62

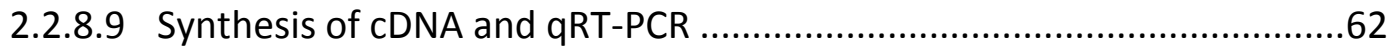

2.2.8.10 Synthesis of oligonucleotides and sequencing ................................63

2.2.9 Molecular biology methods: proteins .......................................................63

2.2.9.1 Isolation of proteins from S. macrospora .........................................63

2.2.9.2 Determination of protein concentration ..........................................63

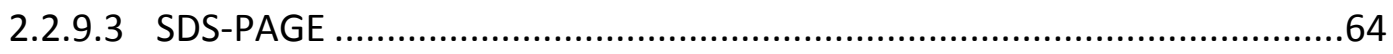

2.2.9.4 Western blotting ......................................................................64

2.2.9.5 Coomassie staining of polyacrylamide gels ...................................64

2.2.9.6 Trypsin digest, stage tipping and sample preparation ........................65

2.2.9.7 Liquid chromatography-mass spectrometry analyses .......................67

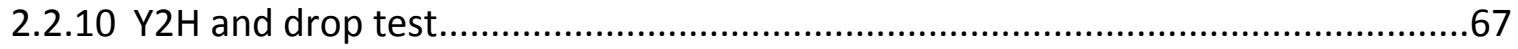

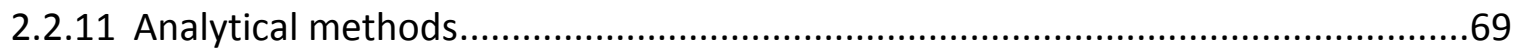


2.2.11.1 Light and fluorescence microscopy ...............................................69

2.2.11.2 DNA and protein sequence analysis............................................. 70

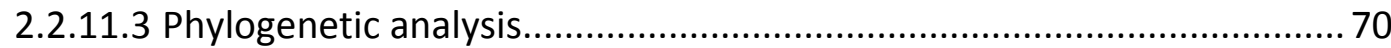

2.2.11.4 LC-MS data analysis: MaxQuant/Perseus and Proteome Discoverer ${ }^{\mathrm{TM}}$.. 70

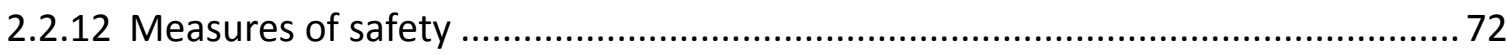

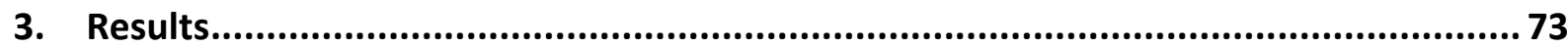

3.1 Pull-down experiments and LC/MS with the STRIPAK proteins PRO11 and SmMOB3 73

3.1.1 LC/MS data analysis with MaxQuant/Perseus identified a putative interaction partner of PRO11 with high confidence ..................................................... 73

3.1.2 LC/MS Data analysis with the Proteome Discoverer ${ }^{\mathrm{TM}}$ identified several putative interaction partners of PRO11 and SmMOB3 .......................................... 76

3.2 Small coiled-coil proteins that associate with the STRIPAK complex in fungi and animals are conserved through their domain structure

3.3 Small CC proteins associated with the STRIPAK complex are conserved within phyla belonging to distant clades ....................................................................... 78

$3.4 \mathrm{SCl} 1$ proteins are conserved in filamentous ascomycetes ...................................... 80

3.5 The sci1 gene is upregulated during sexual development..................................... 81

3.6 Successful generation of a sci1 deletion strain .................................................... 82

3.7 Macroscopic and microscopic analysis of the $\Delta$ scil $_{\text {hyg }}$ strain................................... 84

3.7.1 The scil deletion strain is sterile, produces less protoperithecia than the wt and

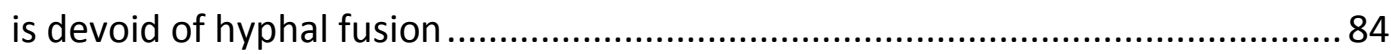

3.7.2 The vegetative growth rate of the $\Delta s c i 1_{\text {hyg }}$ and the $\Delta s c i 1_{\text {hyg }} \Delta$ pro11 strain is

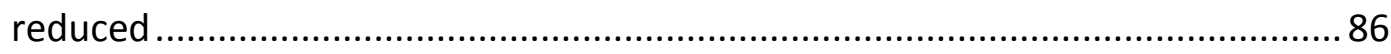

3.7.3 Growth of the $\Delta$ sci $1_{\text {hyg }}$ strain is slightly increased in presence of $\mathrm{H}_{2} \mathrm{O}_{2}$ and $\mathrm{CaCl}_{2}$

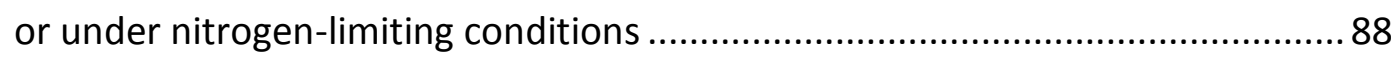

3.8 SCl1 oligomerizes and interacts with PRO11 and PRO45 in yeast two-hybrid

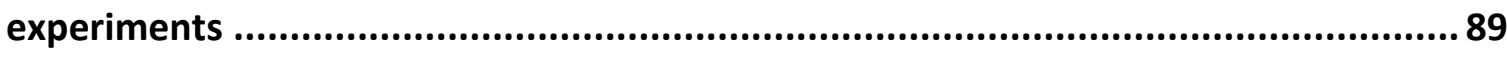

3.8.1 The central region of SCl1 complements the sterile phenotype of $\Delta$ sci $1_{\text {hyg }} \ldots . . . . .91$

3.9 PRO11 was enriched in SCI1eGFP pull-downs .................................................. 92

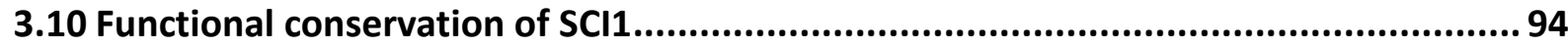


3.10.1 Mammalian FGFR1OP2 and yeast Far3/7 do not complement the sterile phenotype of $\Delta$ sci $_{\text {hyg }}$

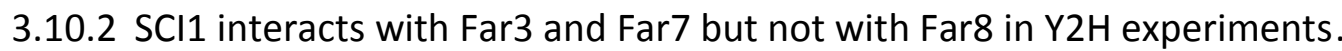

3.11 SCI1 and PRO11 colocalize at the nuclear envelope ............................................ 96

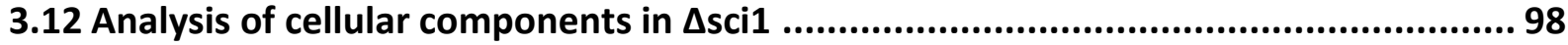

3.12.1 sci1 deletion does not interfere with nuclear transport.................................98

3.12.2 Mitochondria appeared similar in the wt and in sci1 deletion strains .................99

3.12.3 Endoplasmic reticulum in the $\Delta$ sci $_{1 \text { hyg }}$ background appears normal...................99

3.12.4 Actin polymerization and septum formation is not impaired in the sci1 background. 100

3.12.5 Visualization of early and late endosomal marker proteins in the wt and the sci1 deletion background 101

3.12.5.1 The early endosome marker eGFPZtRab5 accumulates in hyphae of the $\Delta$ sci $1_{\text {nat }}$ background 102

3.12.5.2 The late endosome marker eGFPZtRab7 in the $\Delta s c i 1_{\text {nat }}$ background visualizes abnormal vacuolar morphology and behavior 104

4. Discussion 107

4.1 SCI1, a new PRO11 interaction partner was identified in S. macrospora 108

4.2 $\mathrm{SCl} 1$ is a small protein containing coiled-coil domains 109

4.3 Deletion of sci1 causes sterility and prevents hyphal fusion in S. macrospora 110

4.4 sci1 is up-regulated during sexual development 112

4.5 The central region of SCI1 oligomerizes and interacts with PRO11 114

4.6 Functional conservation of STRIPAK complex-associated proteins containing coiled-coil domains 115

4.7 SCl1 is part of the core SmSTRIPAK complex in S. macrospora 116

4.8 SCI1 and PRO11 colocalize at the nuclear envelope 117

4.9 SCl1 might be involved in vesicular transport 119

4.9.1 The STRIPAK complex has a role in ER to Golgi transport 120

4.9.2 The STRIPAK complex is connected to microtubules and Golgi inheritance 122 


\section{Table of Contents}

4.9.3 The SmSTRIPAK complex mediates septation in ascogonia .............................. 124

4.9.4 The STRIPAK complex might be involved in autophagy ................................. 126

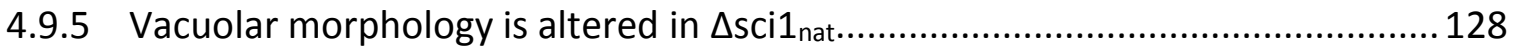

4.9.6 SCI1 and PRO11 connect the SmSTRIPAK complex to vesicular transport and

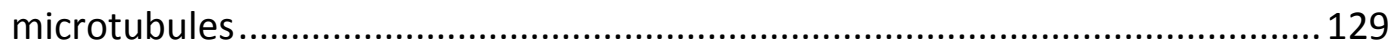

4.10 The STRIPAK complex in S. macrospora is dynamic ....................................... 131

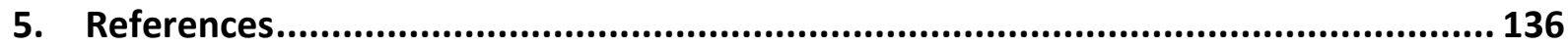

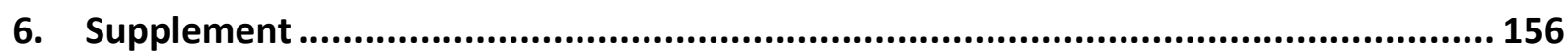

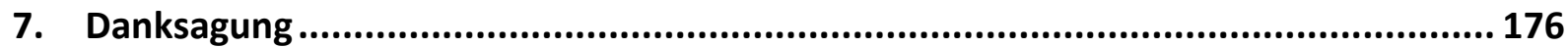

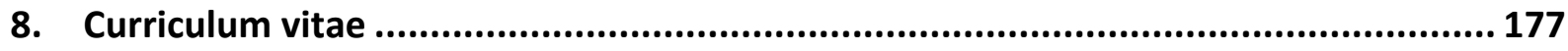




\section{List of figures}

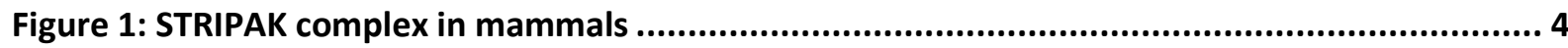

Figure 2: Domains in the mammalian striatin family .................................................................. 7

Figure 3: SIP-SIN-MOR crosstalk regulates cell growth and cell duplication in S. pombe ................ 17

Figure 4: Signaling pathways TORC1/2, Far and MEN in S. cerevisiae ....................................... 19

Figure 5: The dSTRIPAK inhibits Hippo signaling in D. melanogaster......................................... 23

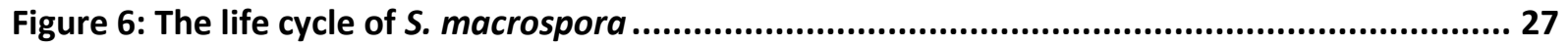

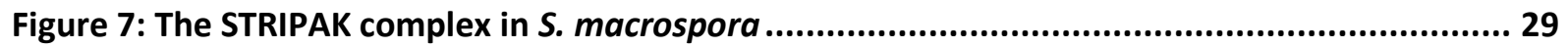

Figure 8: LC/MS data from HA-Trap with HAPRO11 as bait represented in a heat map and a volcano plot 74

Figure 9: Domain structure of small coiled-coil proteins associated with the STRIPAK in fungi and animals. 78

Figure 10: Phylogenetic tree of small CC proteins associated with the STRIPAK ........................... 79

Figure 11: Multiple-sequence alignment of SCI1 proteins in filamentous ascomycetes................. 80

Figure 12: Quantitative reverse-transcription PCR analysis of sci1 ........................................... 82

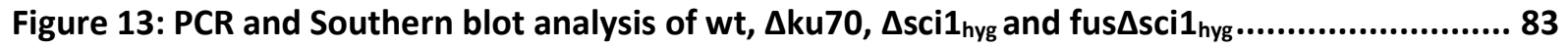

Figure 14: Sexual structures in the wt, $\Delta$ sci1 $_{\text {hyg }}$ and $\Delta$ sci $_{\text {hyg }}:: 5$ 'scilegfp...................................... 85

Figure 15: Vegetative growth rates of the wt, $\Delta$ sci $1_{\text {hyg, }} \Delta s c i 1_{\text {hyg }}:: 5$ 'scilegfp, $\Delta$ sci $1_{\text {hyg }} \Delta$ pro11 and

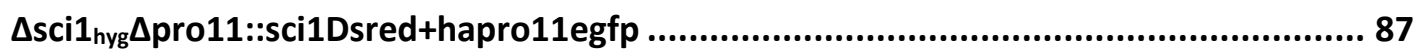

Figure 16: SCI1, PRO11 and PRO45 interact in a yeast two-hybrid analyses.................................90 90

Figure 17: Complementation studies with SCI1 fragments transformed into $\Delta$ sci1 $1_{\text {hyg }} \ldots . . . . . . . . . . . . . . .992$

Figure 18: LC/MS data from SCI1eGFP pull-downs coupled to LC/MS and represented in a heat map

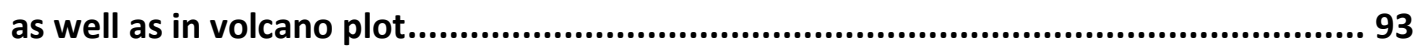

Figure 19: Yeast two-hybrid drop test of SCl1, Far3, Far7 and Far8 .......................................... 95

Figure 20: Localization of SCI1 and PRO11 proteins fused to fluorescent tags visualized in vegetative hyphae of S. macrospora 97

Figure 21: Septation of ascogonia, F-actin polymerization and localization in S. macrospora pro11 and sci1 deletion and wt backgrounds .101

Figure 22: Localization of eGFPZtRab5 in S. macrospora wt::egfpZtrab5 and $\Delta$ sci1 $_{\text {nat }}:$ egfpZtrab5 strain .103

Figure 23: Localization of eGFPZtRab7 in S. macrospora wt::eGFPZtRab7 and $\Delta$ sci1 $_{\text {nat::eGFPZtRab7 }}$ strain 
Figure 24: Speculative representation of SmSTRIPAK proteins required during the life cycle of

S. macrospora

Figure 25: Interactions sites required for SCI1-PRO11, SCI1-SCI1 and SCI1-PRO45 interaction.......115

Figure 26: Schematic representation of the STRIPAK complex in S. macrospora .........................117

Figure 27: The Golgi apparatus, vesicle transport and the Golgi checkpoint in mammals ............120

Figure 28: The SmSTRIPAK complex might be involved in dynein-mediate transport....................124

Figure 29: The STRIPAK complex in S. macrospora might be involved in long and short distance transport of vesicles. .131

Figure 30: Hypothetical model of a dynamic STRIPAK complex in S. macrospora. .134 


\section{List of tables}

Table 1: STRIPAK complex in human, yeasts, fruit fly and filamentous fungi ...............................5

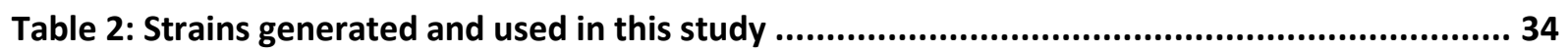

Table 3: List of plasmids used and generated in this study ...................................................... 38

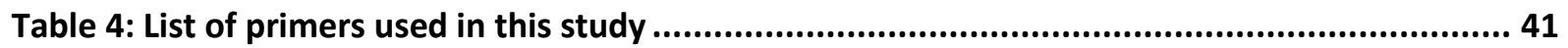

Table 5: Antibodies used for detection of eGFP, DsRED, HA or FLAG-tagged fusion proteins ......... 48

Table 6: Overview of primer pairs used to amplify fragments of sci1 and pRSnat plasmids

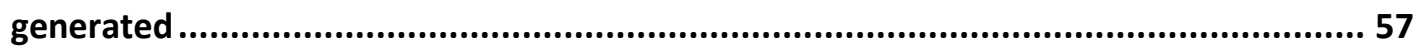

Table 7: Recipes for stacking and resolving polyacrylamide gels .............................................6 64

Table 8: Data from PRO11, SmMOB3 and SCI1 pull-down experiments coupled to LC/MS............ 66 Table 9: Primer pairs used to amplify sci1 fragments for generation of pGBKT7 plasmids encoding

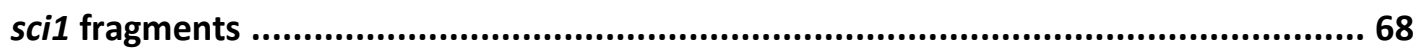

Table 10: Example of a workflow for LC/MS data analysis with MaxQuant/Perseus.................... 71 Table 11: Sum of proteins identified with MaxQuant/Perseus from PRO11 and SmMOB3 pull- downs 75

Table 12: Number of proteins identified with the Proteome Discoverer ${ }^{\mathrm{TM}}$ from PRO11 and SmMOB3

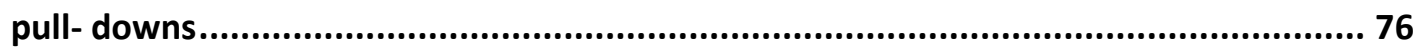




\section{List of figures in the Supplement}

Figure S 1: LC/MS results of HAPRO11eGFP, FLAGSmMOB3 and SmMOB3eGFP pull-downs

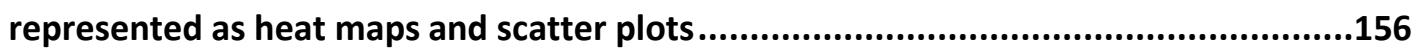

Figure S 2: Heat maps of all proteins identified in LC/MS in 4 different pull-downs.....................157

Figure S 3: Alignment of small coiled-coil orthologs associated with the STRIPAK found in animals, fungi and yeasts. .159

Figure S 4: Neighbor-joining tree and pair-wise comparison of SCl1 amino-acid sequences in filamentous ascomycetes .160

Figure S 5: Genome sequence of sci1 and translated protein sequence. .161

Figure S 6: sci1 fragment after quantitative reverse-transcription PCR. .161

Figure S 7: Verification of the $\Delta s c i 1_{\text {hyg }} \Delta$ pro11 strain by PCR .162

Figure S 8: Growth of wt, pro11, $\Delta$ pro11, $\Delta$ sci $_{\text {hyg }}$ and $\Delta$ sci $1_{\text {hyg }}:: 5$ 'scilegfp under stress conditions 163 Figure S 9: Y2H growth control of full length and fragments of PRO11, SCl1 and full length Far3, Far7 and Far8 .164

Figure S 10: Heat maps of all proteins identified in LC/MS in three SCI1eGFP pull-downs .165

Figure S 11: Western blot using anti-GFP and anti-DsRED for detection of fusion proteins expressed in S. macrospora .166

Figure S 12: Colocalization of SCI1DsRED and HAPRO11eGFP and visualization of nuclei .167

Figure S 13: Nuclei, mitochondria and the ER in wt and $\Delta$ sci1 .167

Figure S 14: PCR and Southern blot analysis of wt, $\Delta s c i 1_{\text {hyg }}$ and, $\Delta s c i 1_{\text {nat }}$ .168

Figure S 15: Fluorescence microscopy of the wt::egfp control strain and strains stained with FM464 . .169

Figure S 16: Detection of eGFPZtRab5 and eGFPZtRab7 using anti-GFP in Western blot analysis ..169 Figure S 17: Localization of the endosomal reporter eGFPZtRab7 and of SmMOB3DsRED, SCI1DsRED and HAPRO11eGFP fusion proteins in vegetative hyphae of $S$. macrospora. 


\section{List of videos in the Supplement/DVD}

Video 1: Nuclei in young hyphae of wt::h2btdTomato

Video 2: Nuclei in young hyphae of $\Delta$ sci $_{\text {hyg }: \text { :h2btdTomato }}$

Video 3: Mitochondria in young hyphae of wt::mitoDsred

Video 4: Mitochondria in hyphae of $\Delta s c i 1_{\text {hyg:: }}$ mitoDsred

Video 5: Actin polymerization in young hyphae of wt::lifeact-tagrfp 174

Video 6: Actin polymerization in hyphae of $\Delta$ sci $_{\text {hyg }}:$ lifeact-tagrfp

Video 7: Structures visualized by early endosome marker eGFPZtRab5 in young hyphae of wt::egfpZtrab5

Video 8: Structures visualized by EE marker eGFPZtRab5 in young hyphae of $\Delta$ sci1 $_{\text {nat }}:$ egfpZtrab5175

Video 9: The EE marker eGFPZtRab5 in young hyphae of $\Delta$ sci1 nat::egfpZtrab5 visualized "cloudy" structures .175

Video 10: Structures visualized by late endosome marker eGFPZtRab7 in young hyphae of wt::egfpZtrab7 .175

Video 11: Structures visualized by the LE marker eGFPZtRab7 in young hyphae of $\Delta$ sci $_{\text {nat }}:$ egfpZtrab7 


\section{List of Abbreviations}

\begin{tabular}{|c|c|}
\hline AA & amino acids \\
\hline$A D$ & activation domain \\
\hline BLAST & basic local alignment search tool \\
\hline BMM & biomalt maize medium \\
\hline $\mathrm{BD}$ & binding domain \\
\hline bp & base pair \\
\hline $\mathrm{CC}$ & coiled-coil \\
\hline CCM3 & cerebral cavernous malformation 3 \\
\hline cDNA & complementary DNA \\
\hline CMS & complex rich medium with $10.8 \%$ sucrose \\
\hline $\mathrm{Ct}$ & threshold cycle \\
\hline CTTNBP2 & cortactin-binding protein 2 \\
\hline CWI & cell wall integrity \\
\hline DIC & differential interference contrast \\
\hline DsRED & encodes red fluorescence protein of Discosoma sp \\
\hline eGFP & enhanced green fluorescent protein \\
\hline ER & endoplasmic reticulum \\
\hline FAR & factor arrest \\
\hline FGFR1 & fibroblast growth factor receptor 1 \\
\hline FGFR1OP2 & fibroblast growth factor receptor oncogene partner 2 \\
\hline gDNA & genomic DNA \\
\hline HAM & hyphal anastomosis \\
\hline IFN & interferon \\
\hline kDa & kilo Dalton \\
\hline LC/MS & liquid chromatography/ mass spectrometry \\
\hline MAP & mitogen-activated protein \\
\hline MAPK & mitogen-activated protein kinase \\
\hline MMS & minimal medium containing soluble starch \\
\hline NDPK & nucleoside diphosphate kinase \\
\hline NE & nuclear envelope \\
\hline NF-KB & nuclear factor kappa-light-chain-enhancer of activated B-cells \\
\hline ORF & open-reading frame \\
\hline PAGE & polyacrylamide gel electrophoresis \\
\hline PCR & polymerase chain reaction \\
\hline PR011 & protoperithecium mutant 11 \\
\hline PRO22 & protoperithecium mutant 22 \\
\hline PRO45 & protoperithecium mutant 45 \\
\hline pT & primary transformant \\
\hline
\end{tabular}




$\begin{array}{ll}\text { qRT-PCR } & \text { quantitative reverse-transcription PCR } \\ \text { REST } & \text { relative gene expression approach } \\ \text { RNA } & \text { ribonucleic acid } \\ \text { SCI1 } & \text { STRIPAK complex interactor 1 } \\ \text { SDS } & \text { sodium dodecyl sulfate } \\ \text { SIKE } & \text { suppressor of IKKE } \\ \text { SLMAP } & \text { sarcolemmal membrane-associated protein } \\ \text { SSDNA } & \text { salmon sperm DNA } \\ \text { SSi } & \text { single spore isolate } \\ \text { SMART } & \text { simple modular architecture research tool } \\ \text { SmGPI } & \text { Sordaria macrospora glycosylphosphatidylinositol } \\ \text { SmKIN3 } & \text { Sordaria macrospora germinal center kinase 3 } \\ \text { SmKIN24 } & \text { Sordaria macrospora germinal center kinase 24 } \\ \text { SmMOB3 } & \text { Sordaria macrospora monopolar spindle one binder 3 } \\ \text { SmPP2AA } & \text { Sordaria macrospora protein phosphatase 2A } \\ \text { SmPP2Ac1 } & \text { Sordaria macrospora catalytic subunit 1 of protein phosphatase 2A } \\ \text { STRIP } & \text { Striatin-interacting protein } \\ \text { STRIPAK } & \text { Striatin-interacting phosphatase and kinase complex } \\ \text { SWG } & \text { Sordaria Westergaards medium } \\ \text { TAP-MS } & \text { tandem affinity purification-mass spectrometry } \\ \text { TBK1 } & \text { TANK-binding kinase 1 } \\ \text { TLR3 } & \text { toll-like receptor 3 } \\ \text { TRIF } & \text { TIR domain-containing adaptor protein } \\ \text { YPD } & \text { yeast extract, peptone, dextrose } \\ \text { wt } & \text { wild type } \\ & \end{array}$




\section{Summary}

The striatin-interacting phosphatase and kinase (STRIPAK) complex is conserved in fungi and animals. The STRIPAK in the filamentous ascomycete Sordaria macrospora, was previously found to be essential for hyphal fusion and fruiting-body development. STRIPAK orthologs in S. macrospora are PRO11 (striatin), PRO22 (STRIP1/2), PRO45 (SLMAP), the serine-threonine phosphatase scaffolding subunit PP2AA and the catalytic subunit SmPP2Ac1 as well as the kinase activator SmMOB3. In this study, PRO11 and SmMOB3 pull-down coupled to liquid chromatography and mass spectrometry (LC/MS) was performed and the LC/MS data was analyzed for putative interaction partners. Among, others an uncharacterized protein was identified as putative PRO11 interaction partner with very high confidence. The uncharacterized protein was named STRIPAK complex interactor 1 (SCI1). SCI1 is an ortholog of small coiled-coil (CC) domain(s) containing proteins found in STRIPAK complexes of yeasts, the fruit fly and mammals. Small CC proteins were discovered in the STRIPAK of Homo sapiens, Drosophila melanogaster, Saccharomyces cerevisiae and Schizosaccharomyces pombe, but their function in the individual STRIPAK complexes is hardly investigated.

This study gives insights into the function of the small CC protein SCI1. The STRIPAK complex was also described in other filamentous fungi and this work shows that $\mathrm{SCl}$ proteins are conserved in filamentous ascomycetes. The sci1 gene is expressed during early developmental stages. Like other STRIPAK genes, sci1 is required for cell to cell fusion and sexual development in S. macrospora. Using yeast two-hybrid experiments oligomerization of SCl1, and direct interaction of SCI1 with PRO11 and PRO45 was shown. In fluorescence microcopy, fluorophoretagged SCI1 and PRO11 fusion proteins showed that SCI1 colocalizes with PRO11 to the nuclear envelope. Additionally, proteins enriched in SCI1eGFP pull-downs were identified with LC/MS and PRO11 was highly enriched. Morphology of nuclei, the ER and mitochondria appeared normal in the S. macrospora sci1 deletion mutant in comparison to the wild type (wt). Also, actin polymerization in vegetative hyphae was similar. However, studies with eGFP-tagged reporter proteins of early and late endosomes of Zymoseptoria tritici, Rab5 and Rab7 respectively, in the S. macrospora sci1 deletion background strongly indicate a defect in vesicle and vacuolar transport. In this study SCI1 was also connected to the Golgi and might be important for vesicle or vacuole transport along microtubules. This is the first study, showing that SCI1 represents a component of the STRIPAK complex in S. macrospora. The results indicate that SCI1 and the 


\section{Summary/Zusammenfassung}

STRIPAK complex are involved in fundamental processes in eukaryotic cells such as transport of vesicles and long distance transport. These processes are important for sexual development, cell fusion and give insights into normal cell function in fungi and animals.

\section{Zusammenfassung}

Der STRIPAK (striatin-interacting phosphatase and kinase)-Komplex ist in Pilzen und Tieren konserviert. In dem filamentösen Askomyceten Sordaria macrospora ist der STRIPAK Komplex an der Hyphenfusion und Entwicklung von Fruchtkörpern beteiligt. Orthologe STRIPAK Proteine in S. macrospora umfassen PRO11 (striatin), PRO22 (STRIP1/2), die Gerüstuntereinheit der SerinThreonin-Phosphatase SmPP2AA, die katalytische Phosphatase Untereinheit SmPP2Ac1 und den Kinase Aktivator SmMOB3. PRO11 und SmMOB3 sowie putative Interaktionspartner wurden affinitäts-chromatographisch angereichert, und massenspektrometrisch untersucht. Von mehreren spezifisch angereicherten Proteinen konnte ein Protein mit sehr hoher Wahrscheinlichkeit als möglicher Interaktionspartner von PRO11 identifiziert werden. Dieses Protein wurde „STRIPAK omplex interactor 1“ (SCI1) genannt. Für SCI1 wurden kleine „çoiledcoil“ (CC) Domänen vorhergesagt. Auch in anderen Organismen assoziieren kleine "CC" Proteine mit STRIPAK Komponenten. Obwohl diese kleinen „CC“ Proteine in STRIPAK Komplexen von Homo sapiens, Drosophila melanogaster, Saccharomyces cerevisiae und Schizosaccharomyces pombe entdeckt wurden, ist ihre Funktion weitgehend unerforscht.

In dieser Studie wurde das kleine „CC" Proteine SCI1 funktionell untersucht. SCI Proteine sind in filamentösen Askomyceten konserviert. In S. macrospora ist das Transkriptionslevel des sci1 Gens in der frühen sexuellen Entwicklung erhöht. Wie auch die anderen STRIPAK Gene, ist sci1 wichtig für die Hyphenfusion und die Entwicklung von Fruchtkörpern. Hefe-Zwei-Hybrid-Analysen zeigten, dass SCI1 oligomerisiert und auch mit PRO11 und PRO45 interagiert. In mikroskopischen Untersuchungen mit SCl1 oder PRO11 Fluoreszenzmarker-Fusionsproteinen, lokalisierten und kolokalisierten SCI1 und PRO11 an der Kernhülle. SCI1 wurde affinitäts-chromatographisch angereichert und die Proben mittels Massenspektrometrie auf potentielle Interaktionspartner untersucht. PRO11 wurde sehr stark angereichert. In mikroskopischen Untersuchungen von Fluoreszenzmarker-Fusionsproteinen, die Kerne, das Endoplasmatische Retikulum, Mitochondrien oder Aktin Polymerisation visualisierten, konnten keine Unterschiede zwischen 
dem Wildtyp und der sci1 S. macrospora Deletionsmutante festgestellt werden. Fluoreszenzmarker-Fusionsproteine eGFPZtRab5 und eGFPZtRab7 aus Zymoseptoria tritici, für frühe und späte Endosomen wurden in den Wildtyp und in die sci1 S. macrospora Deletionsmutante eingebracht um den Vesikeltransport zu untersuchen. Die Ergebnisse weisen drauf hin, dass der Vesikeltransport, welcher auch den Golgi Apparat einschließt in der sci1 Deletionsmutante gestört sind. Weiterhin wird eine Beteiligung von Mikrotubuli vermutet, entweder bei der Ladung von Fracht-Vesikeln auf Mikrotubuli oder direkt beim Tansport.

Diese Studie zeigt zum ersten Mal, dass SCI1 ein Teil des STRIPAK Komplexes in S. macrospora ist. Weiterhin lassen die Ergebnisse dieser Studie darauf schließen, dass SCI1 und somit auch der STRIPAK Komplex in fundamentale Prozesse in eukaryotischen Zellen wie Kommunikation über längere Distanzen und Transport von Vesikeln beteiligt sind. Diese Prozesse stehen im engen Zusammenhang mit sexueller Entwicklung und Zellfusion und sind maßgeblich für das Verständnis normaler Zellfunktion in Pilzen und Tieren. 


\section{Introduction}

Cell development and differentiation in eukaryotes requires tightly regulated signaling pathways, involving corresponding scaffold proteins. Striatins are scaffold proteins in mammals detected in the central and peripheral nervous systems (Benoist et al., 2006, Castets et al., 1996, Castets et al., 2000). They have specialized functions but the presence of striatins in other tissues, for example in fibroblasts, liver cells, skeletal-muscle and cardiac-muscle cells indicates at least one common function (Moreno et al., 2000, Muro et al., 1995, Castets et al., 2000). Striatins are part of the

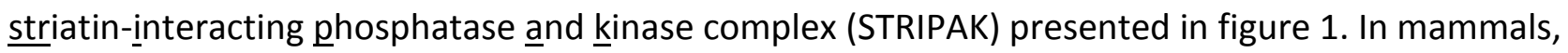
STRIPAK components are the protein phosphatase $2 \underline{\mathrm{A}}$ catalytic subunit (PP2Ac), the scaffolding protein phosphatase $2 \underline{A}$ alpha isoform (PP2AA), the regulatory $B^{\prime \prime \prime}$ subunits (striatins), the monopolar spindle one binder 3 (MOB3), cerebral cavernous malformation 3 (CCM3), striatininteracting proteins 1 and 2 (STRIP1/2) and members of the germinal center kinase III (GCKIII) family of Ste20 kinases. Mutually exclusive interactions allow binding of either the sarcolemmal membrane-asssociated protein (SLMAP) in the sub-complex I or the cortactin- $\underline{b}$ inding protein 2 (CTTNBP2/ CTTNBP2NL) in the sub-complex II (Goudreault et al., 2009).
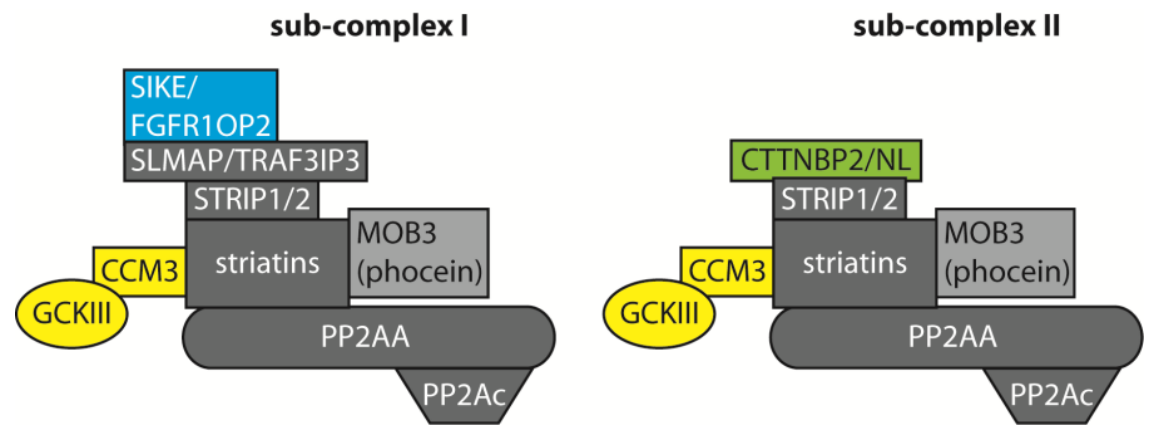

Figure 1: STRIPAK complex in mammals

Schematic representation of both mammalian STRIPAK sub-complexes. PP2AA: scaffolding subunit of phosphatase 2 A, PP2Ac: catalytic subunit of phosphatase $2 \mathrm{~A}$, MOB3 (phocein): monopolar spindle one binder 3, CCM3: cerebral cavernous malformation 3, STRIP1/2: striatin-interacting proteins 1 and 2, GCKIII: germinal center kinase III, CTTNBP2/ CTTNBP2NL: cortactin-binding protein 2, SLMAP: sarcolemmal membrane-associated protein, SIKE: suppressor of IKKE, FGFR1OP2: fibroblast growth factor receptor 1 oncogene partner 2. Modified according to Goudreault et al., 2009.

Furthermore, alternative human STRIPAK components are two related small coiled-coil (CC) proteins, either suppressor of $\underline{\text { IKK }} \underline{\varepsilon}$ (SIKE) or the fibroblast growth factor receptor 1 oncogene 
partner $\underline{2}$ (FGFR1OP2) that associate with the protein SLMAP (Goudreault et al., 2009). However, the function of small CC proteins in the STRIPAK complex is largely unclear.

Table 1: STRIPAK complex in human, yeasts, fruit fly and filamentous fungi

\begin{tabular}{|c|c|c|c|c|c|c|}
\hline Component & 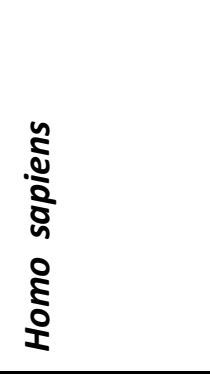 & 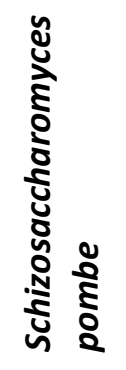 & 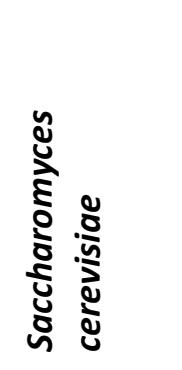 & 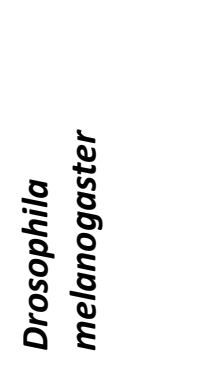 & 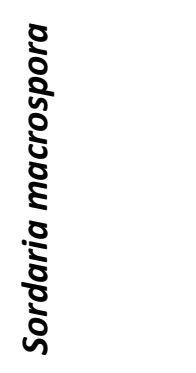 & 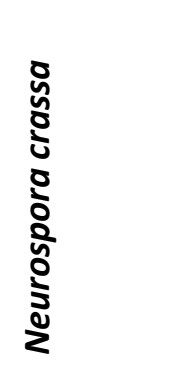 \\
\hline $\begin{array}{l}\text { Phosphatase } \\
\text { scaffolding subunit }\end{array}$ & PP2AA & Paa1 & Tpd3 & Рp2A-29B & SmPP2AA & PP2AA \\
\hline $\begin{array}{l}\text { Phosphatase } \\
\text { catalytic subunit }\end{array}$ & PP2AC & Рpa3 & Ppg1 & Mts & SmPP2Ac1 & PPG-1 \\
\hline $\begin{array}{l}\text { Phosphatase B"' } \\
\text { regulatory subunit } \\
\text { striatins }\end{array}$ & $\begin{array}{l}\text { striatin/ } \\
\text { SG2NA/ } \\
\text { zinedin }\end{array}$ & Csc3 & Far8 & Cka & PRO11 & HAM-3 \\
\hline $\begin{array}{l}\text { Developmental } \\
\text { protein }\end{array}$ & STRIP1/2 & Csc2 & Far11 & CG11526 & PRO22 & HAM-2 \\
\hline $\begin{array}{l}\text { Tail-anchored } \\
\text { protein }\end{array}$ & $\begin{array}{l}\text { SLMAP/ } \\
\text { TRAF3IP3 or } \\
\text { CTTNBP2 }\end{array}$ & Csc1 & Far9/10 & $\begin{array}{l}\text { CG17494 or } \\
\text { CG10915 }\end{array}$ & PRO45 & HAM-4 \\
\hline Kinase & $\begin{array}{l}\text { MST4, } \\
\text { STK24/25 }\end{array}$ & $\begin{array}{l}\text { Ppk11, } \\
\text { Sid1, } \\
\text { Nak1 }\end{array}$ & Kic1, Sps1 & GCKIII & SmKIN3/24 & STK-3/-24 \\
\hline $\begin{array}{l}\text { Small coiled-coil } \\
\text { protein }\end{array}$ & $\begin{array}{l}\text { SIKE/ } \\
\text { FGFR1OP2 }\end{array}$ & Csc4 & Far3/7 & FGOP2 & $\mathrm{SCl} 1$ & NCU04324 \\
\hline Kinase activator & $\begin{array}{l}\text { MOB3 } \\
\text { (phocein) }\end{array}$ & - & - & Mob4 & SmMOB3 & MOB-3 \\
\hline $\begin{array}{l}\text { Dual-targeted } \\
\text { protein }\end{array}$ & - & - & - & - & SmGPI1 & NCU09375 \\
\hline $\begin{array}{l}\text { Neuronal } \\
\text { migration }\end{array}$ & $\mathrm{CCM} 3$ & - & - & CG5073 & - & - \\
\hline
\end{tabular}

Dark blue: Core STRIPAK components found in animals and fungi, Light blue: Small coiled-coil proteins belonging to the core STRIPAK, White: STRIPAK-associated proteins

STRIPAK orthologs are also found in fruit fly, yeasts, and in filamentous fungi, which are wellestablished model organisms for molecular investigations (Table 1). Many studies show that STRIPAK components interact with kinase cascades, regulate development and apoptosis (AshtonBeaucage et al., 2014, Chen et al., 2002, Deng et al., 2016, Dettmann et al., 2013, Lu et al., 2004, Ribeiro et al., 2010). Thus, the STRIPAK complex is involved in various signaling pathways, which 
Introduction

also connects STRIPAK components to human diseases and cancer (Hwang \& Pallas, 2014, Shi et al., 2016). Understanding the molecular interaction of STRIPAK components and their action in signaling pathways will help to understand disease progression and development of adequate treatment for cancer patients and patients suffering from neuronal diseases.

\subsection{Components of mammalian STRIPAK complex}

\subsubsection{Striatins}

Striatins are highly conserved in eukaryotes like metazoa and fungi but not in prokaryotes and plants. In general, striatins are involved in cellular signaling, motor function, and vesicular trafficking (Benoist et al., 2006). In mammals, striatin, $\underline{S} / \underline{G}_{2}$ nuclear autoantigen (SG2NA), and zinedin belong to the striatin family (Castets et al., 1996, Castets et al., 2000, Muro et al., 1995). The first striatin, consisting of 780 amino acids (AA) was identified in the striatum and in motor neurons in the rat brain (Castets et al., 1996). SG2NA was identified as a tumor antigen and at least two SG2NA isoforms are known, SG2Na $\alpha$ containing 713 AAs and SG2NA $\beta$ consisting of 797 AAs (Castets et al., 2000, Muro et al., 1995). However, 6 SG2NA splice variants were described, which are expressed in different developmental stages and are tissue specific (Jain et al., 2015, Sanghamitra et al., 2008). Zinedin was identified by TBLASTN using the full-length striatin sequence (Castets et al., 2000) and localizes in the somato-dendric compartment of hippocampal neurons and dendritic spines (Benoist et al., 2008).

The three mammalian striatins are characterized by a caveolin-binding site, a CC motif, a $\mathrm{Ca}^{2+}$ calmodulin-binding site, and 7 WD40 repeats (Baillat et al., 2001, Gaillard et al., 2006, Gaillard et

al., 2001) (Figure 2). The consensus sequence of caveolin-binding motif is $\Phi x x x x \Phi x x \Phi$, where $x$ represents a random amino acid and $\Phi$ represents an aromatic amino acid (Couet et al., 1997). Caveolins associate with caveolae, which are cell surface invaginations (Parton \& Simons, 2007, Simons \& Toomre, 2000, Boscher \& Nabi, 2012). As a lipid raft protein, caveolin-1 (Cav-1) is enriched in the post-synaptic density (PSD) of post-synaptic compartments of hippocampal neurons and associates with signaling proteins, for example with Ras, G $\alpha$, eNOS and tyrosin kinases (García-Cardeña et al., 1997, Li et al., 1996, Li et al., 1995, Petralia et al., 2003, Song et al., 1996). 
Striatin, SG2NA and zinedin bind to caveolin and thus can associate with membrane microdomains (Gaillard et al., 2001).

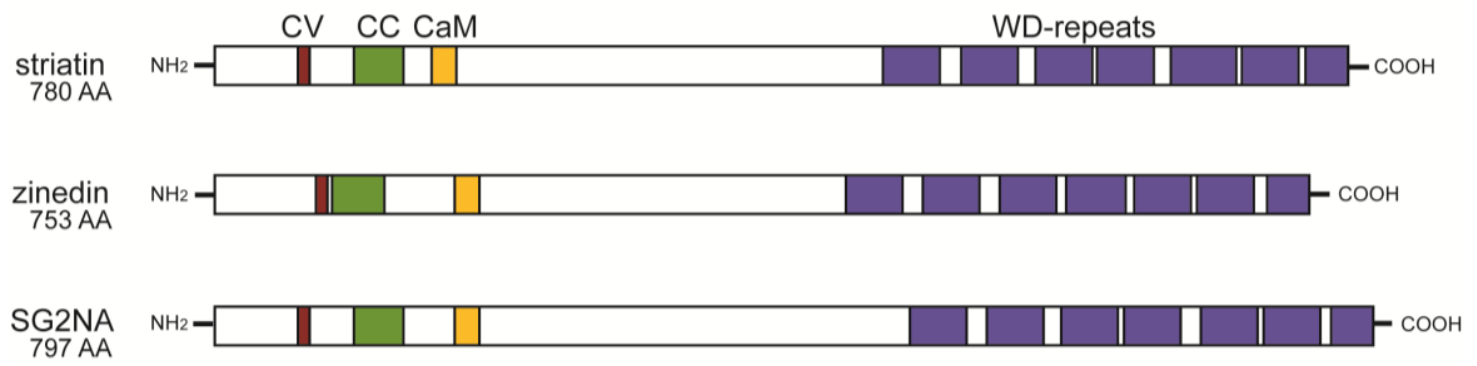

Figure 2: Domains in the mammalian striatin family

Structure of striatin, zinedin and for the isoform $\mathrm{SG} 2 \mathrm{Na} \beta$ is presented. The length of the corresponding proteins is given in the number of amino acids (AA). CV: caveolin-binding domain, CC: coiled-coil domain, CaM: calmodulin-binding domain, WD: tryptophan-aspartic acid repeats. The domains are indicated as predicted but not experimentally verified. Accession numbers: 055106 (Mus musculus, striatin), Q9NRL3 (Homo sapiens, zinedin), Q13033 (H. sapiens, SG2Naß); Modified according to Pöggeler \& Kück, 2004.

Striatin family members are able to oligomerize or heteromerize via their CC domain (Gaillard et al., 2006). CC domains are characterized by a heptad pattern abcdefg, where $a$ and $d$ represent hydrophobic amino acids and $b, c$ and $e-g$ represent polar amino acids (Hodges et al., 1973, McLachlan \& Stewart, 1975, Cohen \& Parry, 1990). In scaffolding proteins, CC domains, which are ampiphilic alpha-helices form a supercoil (Gonzalez et al., 1996, Kammerer, 1997, Lupas, 1996, Yu, 2002).

The CC domain of striatins is contiguous with a basic amphiphilic calmodulin (CaM) -binding site. CaM is a $17 \mathrm{kDa}$ protein, which binds $\mathrm{Ca}^{2+}$ through its EF-hand type helix-loop-helix motifs. The Cterminal calcium-binding lobe is connected via a flexible helix with the $\mathrm{N}$-terminal lobe (Hoeflich \& Ikura, 2002). Striatins interact with CaM in a $\mathrm{Ca}^{2+}$ dependent manner (Castets et al., 1996, Castets et al., 2000, Moreno et al., 2000, Bartoli et al., 1998). CaM-binding might change striatin conformation, which is influenced by $\mathrm{Ca}^{2+}$ concentration and thus regulates the subcellular localization of striatins (Gaillard et al., 2001). Deletion of the CaM-binding domain in human striatin results in increased binding of the GCKIII kinases mammalian STE20-like protein kinase 3 and 4 (MST3 and MST4) to striatin (Gordon et al., 2011). Taken together, it is possible that striatins act as calcium sensors in response to changes in $\mathrm{Ca}^{2+}$ concentration (Benoist et al., 2006). 
Introduction

The C-terminal region of striatins is composed of a WD repeat, whereas $\mathrm{W}$ represents tryptophan and $\mathrm{D}$ represents aspartic acid (Figure 2). The $\mathrm{N}$-terminal region of the WD repeat is characterized by a variable region with 6-96 amino acids followed by a conserved repeat region. The conserved repeats, which are about 40 amino acids long, contain glycine $(\mathrm{G})$ and histidine $(\mathrm{H})$ at the $\mathrm{N}$-terminal region and WD at the C-terminal region (Neer et al., 1994). WD repeats were originally identified in the $\beta$-subunit of heterotrimeric GTP-binding proteins, which are involved in signal transduction across the plasma membrane (Fong et al., 1986). The propeller-like structure of WD repeats brings proteins together and serves as a basis for protein-protein interactions (Hwang \& Pallas, 2014, Lambright et al., 1996, Sondek et al., 1996). Deletion of striatin WD repeats reduces MOB3 binding to striatin (Gordon et al., 2011). Also, WD repeats of mammalian striatin are important for binding to the armadillo repeat domain (ARM) of the adenomatous polyposis coli (APC) protein. APC is expressed in epithelial and fetal cells and functions as multifunctional tumor suppressor that negatively regulates Wnt signaling (Breitman et al., 2008, Midgley et al., 1997). Wnt signaling acts on cell survival, proliferation and differentiation during development (Coudreuse \& Korswagen, 2007, Logan, 2004, Moon et al., 2004, Nelson \& Nusse, 2004).

Striatins are thought to act as scaffolds to bring other proteins together in the STRIPAK complex.

\subsubsection{PP2A}

Phosphorylation is required to inhibit or to activate certain signaling pathways influencing processes like sexual development and proliferation (Farrell, 2014, Grallert et al., 2015). Protein phosphatase $2 \mathrm{~A}$ is a ubiquitously expressed serine/threonine phosphatase, which is connected to cancer, regulates the cell cycle and microtubule dynamics, and plays a role in apoptosis (Baysal et al., 1998, Wang et al., 1998, Wera et al., 1995, Ruvolo et al., 1999, Santoro et al., 1998, NunbhakdiCraig et al., 2007). According to Ruediger et al. (1991) up to 0.3-1\% of the total protein content of a mammalian cell is PP2A.

In mammals, PP2A is a heterotrimeric protein composed of the scaffolding subunit PP2AA (65 kDa), a catalytic subunit PP2Ac (36 kDa) and regulatory subunits PP2AB. The PP2AA subunit consists of two isoforms PP2A $\alpha$ and PP2A $\beta$ that share $85 \%$ sequence identity (da Cruz e Silva \& Cohen, 1987, Hemmings et al., 1990). Furthermore, PP2AA is characterized by 15 huntingtin-elongation- $\underline{A}$ 
Introduction

subunit-TOR (HEAT) repeats, which form a horseshoe-like scaffold for protein-protein interactions (Cho \& Xu, 2007). PP2AA and PP2Ac form a core complex, which associates with regulatory B subunits (Janssens \& Goris, 2001, Janssens et al., 2008). For the catalytic subunit PP2Ac two isoforms are known, PP2Ac $\alpha$ and PP2Ac $\beta$, with highest mRNA abundance in brain and heart tissue (Khew-Goodall \& Hemmings, 1988). PP2Ac $\alpha$ and PP2Ac $\beta$ are encoded by two different genes (Jones et al., 1993). PP2Aca has a 7-10 fold stronger promoter than PP2Ac $\beta$ and is 10 -fold more abundant than PP2Acß (Khew-Goodall et al., 1991).

Of the regulatory $B$ subunits four types exist $\left(B, B^{\prime}, B^{\prime \prime}\right.$ and $\left.B^{\prime \prime \prime}\right)$, which are required for the recruitment of substrates and determine the subcellular localization of proteins associated with PP2A (Goudreault et al., 2009). It was shown that striatin and SG2NA are regulatory B"' subunits interacting with PP2AA and PP2Ac without the presence of other B subunits (Kean et al., 2011, Moreno et al., 2000). Also, striatin and SG2NA seem to be targets of PP2A because inhibition of PP2A with okadaic acid resulted in increased phosphorylation of striatin and SG2NA (Moreno et al., 2000).

The protein phosphatase PP2A is regulated by B subunits and is involved in cell cycle, microtubule dynamics and apoptosis.

\subsubsection{STRIP1/2}

Striatin-interacting proteins 1 and 2 (STRIP1/2), also called FAM40A/FAM40B interact with striatin and mutually exclusively with SLMAP or CTTNBP2/NL (Goudreault et al., 2009) (Figure 1). STRIP1 localizes to the Golgi and depletion of STRIP1 in HeLa cells results in increased DNA content and abnormally shaped or fragmented nuclei (Frost et al., 2012). Also, SLMAP, which was localized to the nuclear envelope and to membranous material around the mitotic spindle interacts with STRIP1 (Frost et al., 2012). Frost et al. (2012) suggested that STRIP1 connects the Golgi and centrosomes and is required for centrosome duplication or spindle assembly.

In prostate carcinoma cells depletion of STRIP1 negatively affects cell spreading and results in increased F-actin in the cell periphery (Bai et al., 2011). Whereas, loss of STRIP2 leads to reduced cell-cell adhesion in HeLa cells (Bai et al., 2011). Taken together, STRIP1/2 might be important for 
Introduction

cytoskeleton organization and function (Hwang \& Pallas, 2014). However, STRIP1/2 are not further characterized in mammals.

STRIP1/2 together with SLMAP bridge the Golgi, the nuclear envelope, and the centrosome and have a role in mitosis.

\subsubsection{SLMAP/TRAF3IPS}

Sarcolemmal membrane-asssociated protein (SLMAP) was found to be a tail-anchored membrane protein in cardiac muscle cells (Wigle et al., 1997). It was postulated that 12 possible splice variants of SLMAP might exist and that they are tissue and development specifically expressed (Wielowieyski et al., 2000, Guzzo et al., 2005, Wigle et al., 1997).

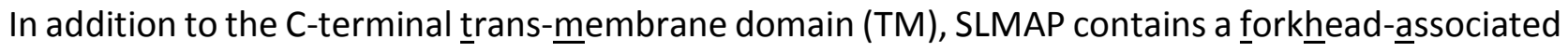
(FHA) domain in the $\mathrm{N}$-terminal region. The FHA domain was shown to be important for interaction with the hippo kinases mammalian STE20-like protein kinase 1 (MST1) and 2 (MST2). SLMAP and MST1/2 interact in a phosphorylation-dependent manner. Moreover, the SLMAP-MST1/2 interaction connects the STRIPAK to hippo signaling (Couzens et al., 2013). SLMAP contains a central coiled-coil region, which is required for SLMAP homo-oligomerization (Guzzo et al., 2005, Guzzo et al., 2004b, Wigle et al., 1997).

Alternative splicing generates SLMAPs with one TM domain, which can be either TM1 or TM2. Thus, alternative splicing makes SLMAPs more versatile and defines subcellular targeting (Byers et al., 2009, Guzzo et al., 2005, Wielowieyski et al., 2000).

SLMAP localizes to centrosomes, is important for cell growth, embryonic myoblast fusion and controls the cell cycle (Guzzo et al., 2004a, Guzzo et al., 2004b). It was also reported to be localized at the ER, the nuclear envelope and mitochondria (Byers et al., 2009, Frost et al., 2012, Guzzo et al., 2005, Guzzo et al., 2004b, Wigle et al., 1997).

Like striatin, SLMAP is a component of the STRIPAK complex (Table 1) and defects in the corresponding genes result in cardiac failure because it is involved in excitation-contraction coupling in cardiomyocytes (Guzzo et al., 2005, Ishikawa et al., 2012, Meurs et al., 2010, Nader et al., 2012).

A protein related to SLMAP is tumor necrosis factor receptor-associated factor 3 interacting protein 3 (TRAF3IP3) (Goudreault et al., 2009) (Figure 1, Table 1). Although, SLMAP and TRAF3IP3 share 
only $22 \%$ sequence identity Goudreault et al. (2009) showed that TRAF3IP3 interacts with striatins, MOB3, STRIP1/2, SLMAP, FGFR1OP2 and SIKE. Neither a FHA nor a TM domain were predicted for TRAF3IP3 but CC regions were predicted for both proteins, SLMAP and TRAF3IP3 (Dadgostar et al., 2003, Goudreault et al., 2009). It was assumed that TRAF3IP3 is either posttranslationally modified or alternatively spliced (Dadgostar et al., 2003).

In mammals, the tumor necrosis factor receptor-associated factor 3 (TRAF3) and its associated protein TRAF3IP3 activate c-Jun N-terminal kinase (JNK) signaling (Dadgostar et al., 2003). TRAF3 also activates nuclear factor-KB (NF-KB), an inducible transcription factor, which allows rapid and transient gene expression (Bista et al., 2010, Hayden \& Ghosh, 2012). NF-KB in mammals induces genes involved in immune and stress response, development, proliferation, differentiation and apoptosis (Baeuerle P. A. \& Henkel, 1994, Hayden et al., 2006).

SLMAP/TRAF3IP3 are STRIPAK complex proteins, which are involved in cell growth, embryonic myoblast fusion, regulation of the cell cycle and development, proliferation, differentiation and apoptosis, respectively.

\subsubsection{CTTNBP2/CTTNBP2NL}

The cortactin- $\underline{\text { b }}$ inding protein 2 and the CCTNBP2 N-terminal like (CTTNBP2/CTTNBP2NL) proteins are part of the mammalian STRIPAK sub complex II (Figure 1, Table 1) (Goudreault et al., 2009). CTTNBP2 is predicted to be $181 \mathrm{kDa}$ in size but also a homolog CTTNBP2NL with a predicted molecular weight of $70 \mathrm{kDa}$ exists in humans. For both proteins an $\mathrm{N}$-terminal CC region was predicted but an ankyrin-repeat motif only for CTTNBP2 (Li et al., 2006, Ohoka \& Takai, 1998). CTTNBP2 was shown to be specifically expressed in brain neurons and very likely involved in neuronal development because defects in CTTNBP2 cause autism spectrum disorders (Ohoka \& Takai, 1998, Cheung et al., 2001, lossifov et al., 2012). CTTNBP2 contains a C-terminal proline-rich region required for interaction with the $\mathrm{SH} 3$ domain of cortactin (cortical actin-binding protein) (Ohoka \& Takai, 1998). In mammals, the STRIPAK was connected to mitogen-activated protein kinase (MAPK)/extracellular signal-regulated kinase (ERK)- signaling and ERK can phosphorylate cortactin (Campbell et al., 1999, Huang et al., 1998, Huang et al., 1997, Shi et al., 2016). 
Introduction

Cortactin is a regulator of the actin cytoskeleton and interacts with F-actin, which regulates the morphology of dendric spines in the central nervous system (Penzes \& Cahill, 2012, Wu \& Parsons, 1993). In sum, CTTNBP2 might affect cortactin phosphorylation and be required for F-actin polymerization (Chen \& Hsueh, 2012, Goudreault et al., 2009). However, while CTTNBP2 was identified as STRIPAK component no cortactin was pulled down with STRIPAK complex components (Goudreault et al., 2009).

\section{CTTNBP2/CTTNBP2NL are part of the mammalian STRIPAK sub complex II and are connected to phosphorylation as well as to regulation of the actin cytoskeleton in neuronal cells.}

\subsubsection{MOB3 (phocein)}

The monopolar spindle one binder 3 (MOB3) protein is a component of the STRIPAK complex and contains a conserved MOB domain (Goudreault et al., 2009, Stavridi et al., 2003, Vitulo et al., 2007). Humans, encode 7 MOB proteins, which are characterized by the 180-200 AA long MOB domain (Chow et al., 2010, Goudreault et al., 2009, Luca \& Winey, 1998, Ponchon et al., 2004, Stavridi et al., 2003, Vitulo et al., 2007). Stavridi et al. (2003) assumed that the negatively charged surface potential of MOB1 might be required to interact with kinases containing conserved basic regions and thus activates kinases electrostatically.

Structural parts of the MOB domain are partially conserved in MOB3. However, MOB3 is the most divergent clade within the MOB family and it was assumed that MOB3 has another function than the other MOB proteins (Stavridi et al., 2003, Vitulo et al., 2007). The STRIPAK complex component MOB3 is a 225 AA protein and localizes to the cytosol, to membranes and the Golgi. It was localized to somato-dendritic compartments and was shown to interact with striatin, SG2NA and zinedin (Baillat et al., 2001).

MOB3 contains a region homologous to the small chain signature of $\sigma$ subunits of clathrin adaptor protein (AP) complexes (Baillat et al., 2001). The AP complexes consist of two large subunits, one medium subunit and one small subunit, where the small $\sigma$ subunit stabilizes the AP complex mediating the formation of vesicles as well as selective packing of the cargoes (Collins et al., 2002, Kirchhausen, 1999, Park \& Guo, 2014, Schmid, 1997) The adaptor complexes AP-1, AP-2 and AP-3 are involved in clathrin-mediated vesicular traffic and endocytosis. Clathrin-mediated endocytosis 
Introduction

is not only required for nutrient uptake but also required to remove receptors, integral proteins or lipids from the plasma membrane. The AP-1 complex is recruited to trans-Golgi coated vesicles whereas the AP-2 complex associates with plasma membrane coated vesicles (Keen, 1990, Robinson, 1992, Park \& Guo, 2014). While, the AP-3 complex is involved in transport from transGolgi to lysosomes (Gu et al., 2001, Le Borgne et al., 1998).

MOB3 was also shown to interact with epidermal growth factor receptor substrate 15 (EPS15) and nucleoside-diphosphate kinase NDPK (Baillat et al., 2002). EPS15 interacts with AP-2 and is involved in clathrin-mediated endocytosis (Benmerah et al., 1996). NDPK interacts with dynamin I mediating the abscission of vesicles from plasma membranes (Antonny et al., 2016, Baillat et al., 2002, Ferguson \& De Camilli, 2012).

The secretion blocking agent $\underline{b}$ refeldin $\underline{A}$ (BFA) inhibits small G proteins of the $\underline{A} D P$-ribosylation factor (Arf) family, which organize vesicular trafficking (Donaldson et al., 1992). Localization of

MOB3 and SG2NA in rat neurons was altered upon exposure to BFA. Thus, interaction of MOB3 with Eps15 or NDPK and altered localization of MOB3 upon BFA treatment suggests that MOB3 is involved in vesicular traffic (Baillat et al., 2002).

MOB3 interacts with striatins and is thought to be involved in vesicular transport.

\subsubsection{Germinal center kinases and CCM3}

Germinal çenter kinases (GCK) are regulators of cell growth, apoptosis, neuronal differentiation, polarization, migration, cell growth and stress response (Delpire, 2009, Sugden et al., 2013). The STRIPAK complex in mammals contains four possible serine/threonine kinases, MST4, MST3 (STK24), serine/threonine kinase 25 (STK25 also called SOK1/YSK1), which belong to the germinal center kinase III (GCKIII) subfamily and misshapen/Nck-interacting kinase (NIK)-related kinase 1 (MINK1), which belongs to the GCKIV subfamily (Gordon et al., 2011, Goudreault et al., 2009, Hyodo et al., 2012, Kean et al., 2011) (Table 1). All four kinases contain an N-terminal catalytic domain but MINK1 additionally contains a citron homology domain (Delpire, 2009).

MST4, MST3 and STK25 interact with cerebral cavernous malformation 3 (CCM3), which also associates with the STRIPAK complex (Fidalgo et al., 2010, Goudreault et al., 2009) (Figure 1). 
Introduction

\subsubsection{MST3}

MST3 contains a nuclear export and localization signal. Cleavage of MST3 by caspase activates the kinase, which translocates to the nucleus and results in induction of apoptosis. Four striatin point mutants were unable to bind PP2A, which resulted in hyperphosphorylated and active MST3. Hence, PP2A was shown to be a negative regulator of MST3 (Gordon et al., 2011). Also, SG2NA was suggested to recruit PP2A and to inhibit MST3 activation suppressing apoptosis (Chen et al., 2014).

\subsubsection{MST4 and STK25}

The programmed cell death $10(P D C D 10)$ gene product CCM3 is connected to apoptosis and tumor progression. Mutations in the PDCD10 gene result in cerebral cavernous malformations (Ma et al., 2007). It was shown that CCM3 increases MST4 activity inducing the MAPK/ERK pathway stimulating cell growth (Ma et al., 2007). Further, MST4 was found in a trimeric complex with SG2NA and CCM3, which recruits MST4 to the STRIPAK (Kean et al., 2011).

STK25 has a role in neuronal migration, is activated by oxidative stress and can induce apoptosis (Matsuki et al., 2013, Pombo et al., 1996, Pombo et al., 1997, Zhang et al., 2014). CCM3 interacts with STK25 to regulate MAP kinase/ERK activity under oxidative stress (Zhang et al., 2012). STK25, like MST3, can enter the nucleus to induce cell death (Nogueira et al., 2008).

\subsubsection{MINK}

MINK1 is abundant in the brain and is upregulated during postnatal cerebral development. It is an activator of the JNK pathway but not of the MAP kinase /ERK pathway (Dan et al., 2000). Zinedin together with PP2A was shown to deactivate MINK1 by dephosphorylation (Hyodo et al., 2012). Phosphorylated and catalytic active MINK1 is found in mitosis whereas PP2A is inhibited during mitosis (Wurzenberger \& Gerlich, 2011). Thus, inactivation of MINK1 through interaction with zinedin and PP2A might regulate abscission in the last step of cytokinesis (Hyodo et al., 2012).

The GCKs MST3, MST4, STK25 and MINK, which control cellular processes like cell differentiation, cytokinesis, and apoptosis associate with the STRIPAK complex in mammals. 
Introduction

\subsubsection{SIKE/FGFR1OP2}

Two small coiled-coil proteins, suppressor of $\underline{I K K} \underline{\varepsilon}$ (SIKE) and fibroblast growth factor receptor 1 oncogene partner $\underline{2}$ (FGFR1OP2), were identified in the mammalian STRIPAK sub-complex I (Table 1). SIKE and FGFR1OP2 are predicted to contain 3 and 4 CC domains, respectively (Marion et al., 2013, Lin et al., 2010, Grand et al., 2004). The sequences of SIKE and FGFR1OP2 share 56\% identity but SIKE contains additionally 6 TANK-binding kinase 1 phosphorylation sites in its C-terminal region (Marion et al., 2013).

\subsubsection{SIKE}

Human SIKE is a physiological suppressor of the serine/threonine-protein kinases IKKE and TANKbinding kinase 1 (TBK1) that play important roles in regulating immune response via transcription factors belonging to the interferon regulatory factor family and the NF-kB family, which is involved in cell survival, development and proliferation (Hayden \& Ghosh, 2012, Huang et al., 2005a). SIKE is also a negative regulator of pathological cardiac hypertrophy because SIKE interacts with TBK1 and inhibits the TBK1-RAC-alpha serine/threonine-protein kinase AKT (AK strain transforming) pathway (Deng et al., 2016). However, it is not clear if SIKE connects immune response or the AKT pathway with the STRIPAK complex or if it acts independently of the STRIPAK (Hwang \& Pallas, 2014).

\subsubsection{FGFR1OP2}

The wound inducible transcript-3.0 (wit-3.0) encodes the SIKE-related protein FGFR1OP2 and was identified in cDNA from an oral wound library (Lin et al., 2010). In a 75-year-old male, the wit-3.0 $\mathrm{N}$-terminal domain encoding CC sequence inserted into the fibroblast growth factor receptor 1 (FGFR1) gene caused ligand independent dimerization of FGFR1. The dimerization led to T-cell lymphoblastic lymphoma and myelogenous leukemia. Thus, the gene was named FGFR1 oncogene partner 2 (FGFR1OP2). FGFR1OP2 influences cell migration and is selectively expressed in fibroblasts, which are in contact with cells in the active margin approximation. Overexpression of FGFR1OP2 in oral wound fibroblasts had increased collagen gel contraction ability and thus significantly accelerated skin wound closure with minimum scaring of oral wounds (Lin et al., 2010).

\section{Despite that SIKE and FGFR1OP2 were identified to be STRIPAK components, their function within the STRIPAK complex is not investigated in mammals.}


Introduction

\subsection{STRIPAK-like complexes in yeasts and in Drosophila melanogaster}

STRIPAK orthologs were also identified in the fission yeast Schizosaccharomyces pombe and in the baker's yeast Saccharomyces cerevisiae. In S. pombe, the STRIPAK complex is called SIN-inhibitory phosphatase complex (SIP) whereas in S. cerevisiae the STRIPAK complex is called factor arrest (Far) complex (Table 1). The SIP complex in fission yeast and interaction with other signaling pathways will be explained first. Subsequently, the Far complex in the baker's yeast and crosstalk to other signaling pathways will be elucidated followed by the STRIPAK complex and its signaling interactions in Drosophila melanogaster.

\subsubsection{The SIP-SIN-MOR crosstalk in S. pombe}

Cytokinesis in the fission yeast is regulated by the septation initiation network (SIN) (Guertin et al., 2002, Krapp \& Simanis, 2008, McCollum \& Gould, 2001). The polo kinase 1 (Plo1) is one protein that regulates SIN (Tanaka et al., 2001). Loss of PLO1 function leads to cell arrest in mitosis and cells without septa (Ohkura et al., 1995). To ensure correct cytokinesis, components of the SIN localize asymmetrically to the spindle-pole bodies (SPBs).

The SIN-inhibitory phosphatase (SIP) complex was found to be a weak inhibitor of the SIN, which promotes SIN asymmetry at SPBs and regulates cytokinesis (Singh et al., 2011). In S. pombe, the SIP complex indicated in gray in figure 3 , is an ortholog of the mammalian STRIPAK complex. S. pombe expresses the STRIPAK orthologs, component of SIP complex 3 (Csc3) (striatin), Csc2 (STRIP1/2), protein phosphatase $\underline{P} P 2 \underline{A}$ regulatory subunit $\underline{A}$ (Paa1), Ppa3 (PP2Ac1) and Csc1 (SLMAP). Furthermore, the small CC protein Csc4 was identified in the STRIPAK complex of S. pombe (Singh et al., 2011) (Table 1).

The SIN complex, indicated in blue in figure 3, contains three kinases: the cyclin-dependent kinase cell division cycle 7 (Cdc7), the GC kinase septum initiation defective 1 (Sid1) and the nuclear $\underline{\text { dbf2- }}$ related (NDR) kinase Sid2. The SIN complex also contains the small GTPase septum promoting GTPase 1 (Spg1), two scaffold proteins Cdc11 and Sid4, which bind via pombe pole component 89 (Ppc89) to SPBs and the adaptor proteins Cdc14 and Mob1. The scaffold protein Sid4 interacts with Ppc89 and connects the SIN to SPBs (Rosenberg et al., 2006). During interphase GTPase Spg1 and the GAP proteins Cdc16 and Byr4 are located on the SPBs to keep Spg1 inactive but are removed during metaphase and allow binding of Cdc7 to SPBs (Fankhauser \& Simanis, 1994). 


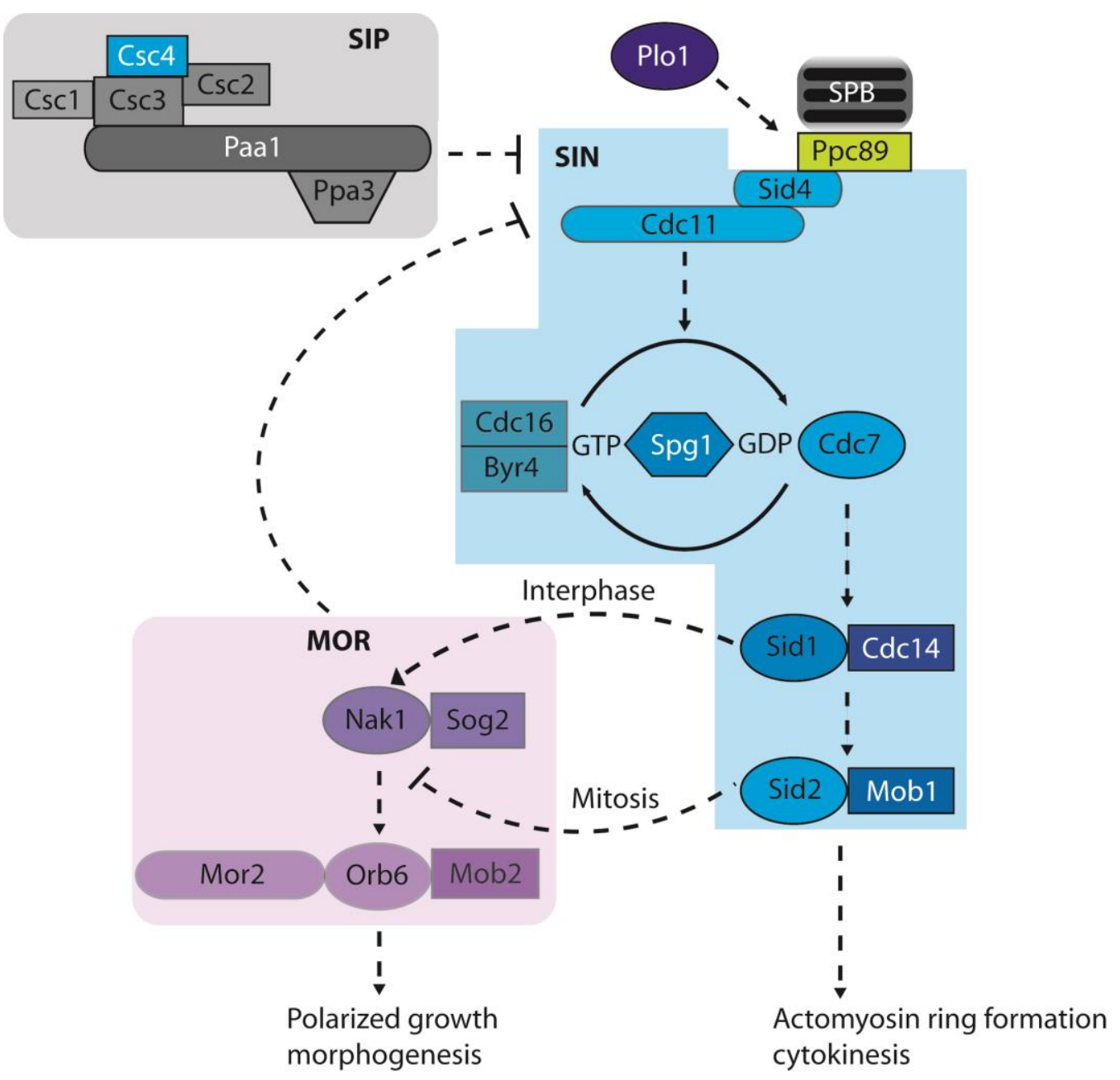

Figure 3: SIP-SIN-MOR crosstalk regulates cell growth and cell duplication in S. pombe

The SIN-inhibitory phosphatase (SIP) complex is suggested to negatively regulate the septation initiation network (SIN) because mutants with nonfunctional SIP genes show a hyperactive SIN phenotype with premature septum formation and cells without nuclei. In general, the polo kinase Plo1 is an activator of the SIN, which regulates actomyosin ring formation and cytokinesis in S. pombe. The morphogenesis Orb6 network (MOR) pathway is negatively regulated by the SIN during mitosis. Sid2 blocks the interaction of Nak1 and the scaffolding protein Mor2 to prevent polarized growth during mitosis. However, SIN can also induce MOR during interphase. Cdc7 via Sid1 activates Nak1 to promote polarized growth. MOR also negatively regulates SIN via Nak1, which persists on SPBs to prevent premature SIN activation. Dash lines: not completely described interaction/pathway or pathway simplified. Continuous lines: direct interactions. Modified from Krapp \& Simanis (2008) and Gupta \& McCollum (2011). Detailed explanation and abbreviations are given in the text.

SIN signaling is transmitted via Cdc7, Sid1-Cdc14 and Sid2-Mob1 to promote actomyosin ring maturation, constriction and septation (Chang \& Gould, 2000, Fankhauser \& Simanis, 1994, Guertin et al., 2000, Hou et al., 2000, Krapp et al., 2001, Salimova et al., 2000, Sparks et al., 1999) (Figure 3). Loss of SIN genes reduces or prevents septation and leads to multinucleated and elongated cells 
whereas a hyperactive SIN leads to premature septum formation and compartments without nuclei (Fankhauser et al., 1993, Fankhauser \& Simanis, 1993, Li et al., 2000, Minet et al., 1979, Schmidt et al., 1997). Localization of SIP components Csc1, Csc2, Csc3 and the PP2A regulatory subunit A (Paa1) showed that all four proteins localize to SPBs and Csc2 and Csc3 were additionally localized to the nuclear envelope. $\mathrm{Bi}$ - and uni-nucleated cells with defects in septation (two or more septae) were reported when SIP function was abolished. Thus, SIP was shown to be a negative regulator of SIN (Singh et al., 2011). Furthermore, SIN is negatively regulated by the morphogenesis Orb6 network (MOR) indicated in purple in figure 3. The MOR pathway includes the germinal center II (GC) kinase Nak1 with its binding partner the leucine-rich repeat protein Sog2 (Huang et al., 2003). Nak1 activates the NDR kinase Orb6, which interacts with the scaffold protein morphological round 2 (Mor2) and with its coactivator the Mob1-related protein Mob2 (Figure 3). During interphase MOR is required for polarized growth and controls cell morphology. Via Cdc7 and Sid1, Nak1 activity is upregulated (Kanai et al., 2005). It was suggested that MOR is inhibited by the SIN during mitosis to prevent actin competition (Gupta \& McCollum, 2011). The NDR/LATS family kinase Sid2 of the SIN pathway can phosphorylate Nak1, blocking the Nak1-Mor2 interaction and removing Nak1 from SPBs, which in turn activates SIN. It was concluded that Nak1 might reside on SPBs to prevent premature SIN activation (Gupta et al., 2013).

Taken together, SIN inhibits MOR during mitosis to ensure cell division but also enhances MOR during interphase when polarized cell growth takes place. Moreover, SIP seems to be involved in tight regulation between cell duplication and cell growth.

\subsubsection{The Far complex in Saccharomyces cerevisiae}

\subsubsection{The Far complex in S. cerevisiae is involved in pheromone-mediated cell cycle arrest}

Haploid S. cerevisiae cells sense their mating partner via mating pheromones and by a $\mathrm{G}$ proteincoupled serpentine receptor leading to the activation of the mitogen-activated protein kinase cascade and cell cycle arrest. The pheromone response is transmitted to facilitate mating and promotes expression of mating-specific genes (Elion, 2001, Leberer et al., 1992, Nomoto et al., 1990, Whiteway et al., 1989). 


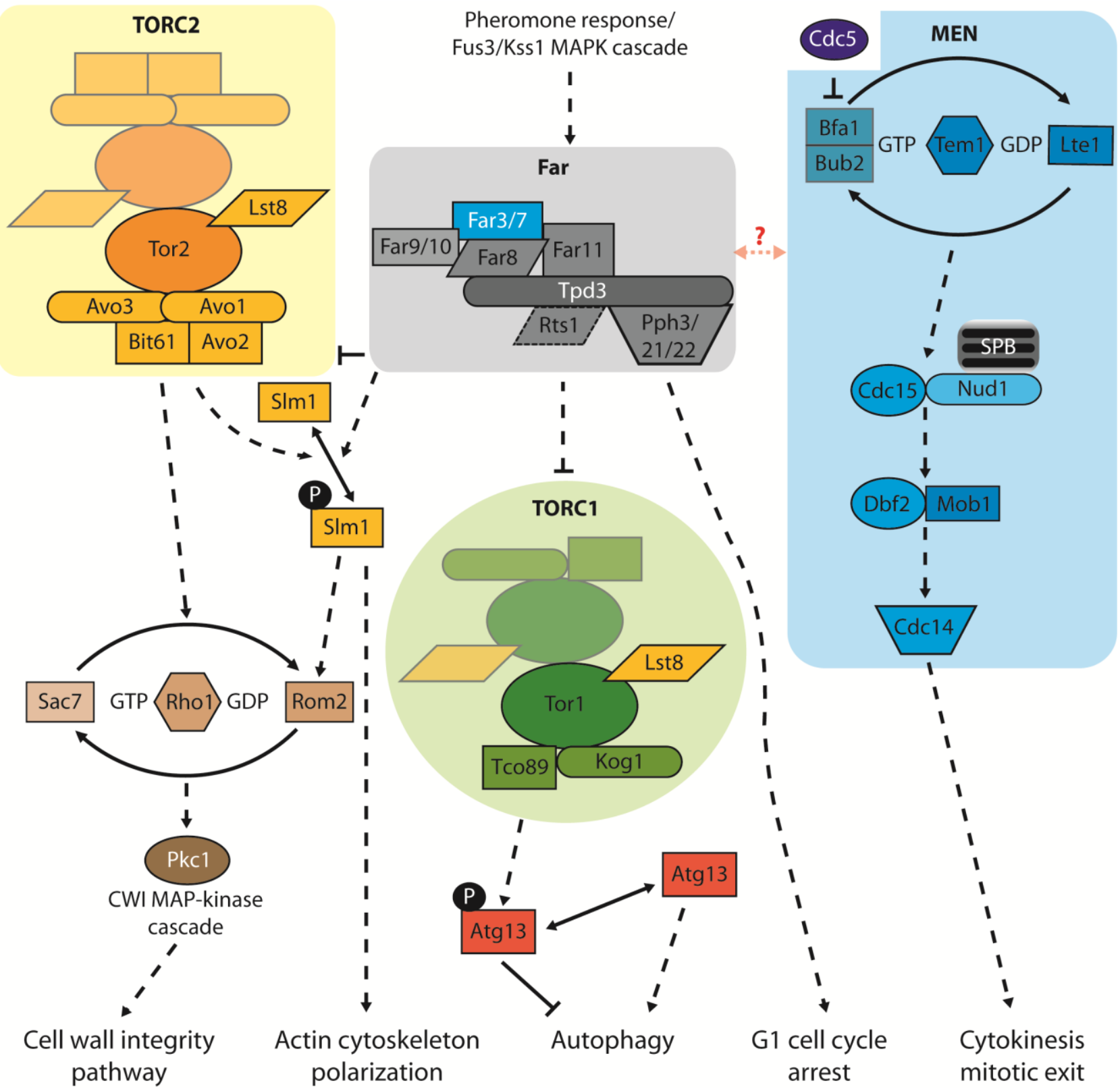

Figure 4: Signaling pathways TORC1/2, Far and MEN in S. cerevisiae

Schematic representation of the Far complex interacting with signaling pathways target of rapamycin complex 1 (TORC1), TORC2, cell wall integrity (CWI) pathway and mitotic exit network (MEN) in S. cerevisiae. Pheromones activate the Fus3/Kss1 mitogen-activated protein kinase cascade. The Far complex is thought to prevent premature recovery from pheromone induced G1 arrest until cells fuse and then enter meiosis. TORC2 signaling acts on the Rho signaling/CWI pathway and actin cytoskeleton polarization via phosphorylation of SIm1 but the Far complex can mediate dephosphorylation of SIm1 and antagonizes actin cytoskeleton polarization. Via SIm1 Far complex also regulates the cell wall integrity pathway. The Far complex negatively regulates TORC1 signaling leading to dephosphorylation of Atg13 which induces autophagy. The Far complex is suggested to interact with the MEN regulating cytokinesis and mitotic exit but the mode of action is unclear. Dash lines: not completely described interaction/pathway or pathway simplified. Continuous lines: direct interactions. Modified from Bardin and Amon (2001), Hergovich et al. (2008) Kemp \& Sprague (2003), Pracheil et al. (2012), Pracheil \& Liu (2013). Detailed explanation and abbreviations are given in the text. 
In S. cerevisiae, the factor arrest (Far) complex was shown to be involved in pheromone-induced G1 cell cycle arrest and shares the orthologs Far8 (striatin), Far9/Far10 (SLMAP) and Far11 (STRIP1/2) with the STRIPAK complex (Goudreault et al., 2009, Kemp \& Sprague, 2003, Lai et al., 2011) (Table 1). The phosphatase scaffolding subunit in S. cerevisiae is called Tpd3 and interacts with Far11 as well as with the catalytic protein phosphatase subunits Pph3, Pph21, Pph22 (LisaSantamaría et al., 2012) (Figure 4, Table 1). Interestingly, the two small cc proteins Far3 and Far7 are present in the Far complex (Kemp \& Sprague, 2003, Lai et al., 2011). Far3 interacts with Far7 and Far8 to form a sub complex recruiting Far9 and Far10 to the endoplasmic reticulum (ER) (Pracheil \& Liu, 2013).

Yeasts with mutations in FAR genes are resistant to pheromone-induced G1 arrest, thus it was suggested that the Far complex prevents premature recovery from G1 arrest to ensure that mating cells have enough time to fuse before they enter meiosis.

\subsubsection{The Far complex regulates cell growth, autophagy and actin polarization}

The Far complex in S. cerevisiae was connected to target of rapamycin complex (TORC) signaling (Lisa) (Figure 4). The TORC1 is composed of the Tor1 kinase, the modulator lethal with sec thirteen 8 (Lst8) protein, the 89-kDa subunit of Ior complex one (Tco98) and the scaffolding protein kontroller of growth 1 (KOG1) and is involved in cell growth and autophagy. On the other hand, the second rapamycin insensitive complex of S. cerevisiae, TORC2, is involved in cell cycle-dependent polarization of the actin cytoskeleton (Cybulski \& Hall, 2009, Schmelzle \& Hall, 2000, Wullschleger et al., 2005) (Figure 4).

Rapamycin treatment leads to TORC1 inhibition, dephosphorylation of Atg13 and, finally to initiation of autophagy. However, dephosphorylation of Atg13 did not take place in FAR11 deletion mutants, not even when they were treated with rapamycin (Lisa-Santamaría et al., 2012). Far11 might inhibit TORC1 directly or by an alternative way inhibiting the serine/threonine kinase target of rapamycin (Tor1) leading to autophagy (Lisa-Santamaría et al., 2012, Wullschleger et al., 2005). TORC2-deficient mutants have been shown to be lethal but Far deletion mutants suppress the lethal phenotype partially (Pracheil et al., 2012). The TORC2 contains the kinase Tor2, the scaffolding proteins a adheres-voraciously-to-target-of-rapamycin-2 protein 1 (Avo1) and 3 (Avo3), 
the modulator Lst8 and the adaptor proteins Avo2 and 61-kDa-binding partner of Tor2 (Bit61) (Wullschleger et al., 2005). Depletion of the STRIP1/2 ortholog Far11 in Avo3 mutants suppressed actin polarization defects (Baryshnikova et al., 2010). Thus, the phosphatidylinositol-4-phosphate 5-kinase-binding protein synthetic lethal with $\underline{M} s s 4$ (multicopy suppressor of $\underline{\text { Stt}} 4$ mutation) 1 (SIm1) is a TORC2 substrate, which is involved in actin cytoskeleton organization and might be dephosphorylated by the Far11-PP2A complex associated with the B'-regulatory subunit rox three suppressor 1 (Rts1) (Pracheil et al., 2012). Thus, it was suggested that the Far complex acts downstream of the TORC2 pathway, negatively regulates TORC2 signaling, and is involved in actin polarization (Baryshnikova et al., 2010, Pracheil et al., 2012) (Figure 4).

TORC2 signaling also activates the Ras homolog 1 (Rho1) GTPase switch. hㅡo1 multicopy suppressor 2 (Rom2) is the GDP-GTP exchange factor and suppressor of actin 7 (Sac7) the activating protein of Rho1. The Rho1-GTP activated protein kinase $\underline{C} 1$ (Pkc1) pathway, which includes also the Pkc1-activated cell wall integrity (CWI) MAP-kinase cascade pathway controls actin distribution and cell wall extension (Levin, 2011) (Figure 4).

The deletion of ROM2 in TORC2-deficiency mutants leads to loss of actin polarization in theses mutants, whereas, the deletion of FAR11 and SAC7 results in polarization of the actin cytoskeleton. Thus, it was suggested that Far11 acts downstream of TORC2 but upstream of Rho GTPase signaling mediating phosphorylation of SIm1 (Pracheil et al., 2012) (Figure 4). Nevertheless, it remains unclear if two regulatory B subunits, B' Rts1 and B"' Far8, can associate with PP2A at the same time. It can be speculated that the Far complex exists in parallel to a Far11-PP2A-Rts1 complex and a crosstalk between theses complexes might influence TORC1/2 signaling.

The Far complex regulates TORC1/2 signaling as well as autophagy, actin polymerization and the cell wall integrity pathway.

\subsubsection{MEN regulates mitotic exit and cytokinesis in S. cerevisiae}

The SIN in S. cerevisiae is called mitotic exit network (MEN). The MEN is a GTPase signaling cascade and is regulated by the spindle-pole body (SPB) localized small GTPase termination of $\underline{M}$ phase 1

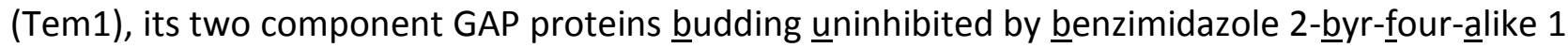
(Bub2-Bfa1) and its putative exchange factor low temperature essential 1 (Lte1). The polo kinase 
Cdc5 phosphorylates and inactivates the Bub2-Bfa1 MEN inhibiting complex rendering Tem1 active, thereby initiating MEN signaling. MEN signaling is transmitted through the hippo-related kinase Cdc15 and the serine/threonine kinase dumbbell former 2 (Dbf2), which associates with its adaptor protein Mob1. Cdc15 interacts with the scaffolding protein Nud1 connecting MEN complex proteins with SPBs (Gruneberg et al., 2000) (Figure 4). Therefore, the SIN in S. pombe as well as the MEN in S. cerevisiae are both connected to SPBs (Figure 3 and Figure 4).

The Dbf2-Mob1 complex phosphorylates the tyrosine protein phosphatase Cdc14, which inactivates mitotic cyclins by dephosphorylation to exit mitosis (Bardin \& Amon, 2001, Bosl \& Li, Meitinger et al., 2012, Mohl et al., 2009). Both, Cdc15 and Dbf2-Mob1 are involved in actomyosin ring contraction but only Dbf2-Mob1 regulates cytokinesis (Meitinger et al., 2012, Meitinger et al., 2010, Menssen et al., 2001). Crosstalk between the Far complex and the MEN signaling in S. cerevisiae is suggested but the mode of action is unclear.

In S. pombe, the SIN is negatively regulated by the STRIPAK ortholog SIP, thus it is possible that the Far complex also regulates mitotic exit and cytokinesis through MEN signaling in S. cerevisiae.

\subsubsection{STRIPAK complex in Drosophila melanogaster}

The STRIPAK in Drosophila melanogaster (dSTRIPAK) is involved in development, more precisely, in embryonic dorsal closure and apoptosis of cells in wing imaginal disks (Chen et al., 2002). The dSTRIPAK contains the striatin ortholog Cka, the phosphatase scaffolding subunit Pp2A-29B and the phosphatase catalytic subunit Mts as well as the STRIP1/2 ortholog CG11526, the SLMAP ortholog CG17494, the CTTNBP2 ortholog CG10915, the kinase activator Mob4, the kinase GCKIII and the CCM3 ortholog CG5073 (Ashton-Beaucage et al., 2014, Ribeiro et al., 2010) (Figure 5, Table 1).

\subsubsection{Hippo signaling in D. melanogaster}

In D. melanogaster the dSTRIPAK complex inhibits Hippo signaling (Ribeiro et al., 2010). The Drosophila ortholog of FGFR1OP2, named FGOP2, associates with the striatin ortholog Cka negatively regulating Hippo signaling to prevent restriction of tissue growth and apoptosis during development (Ribeiro et al., 2010). Moreover, Hippo signaling regulates F-actin polymerization and migration (Lucas et al., 2013). 


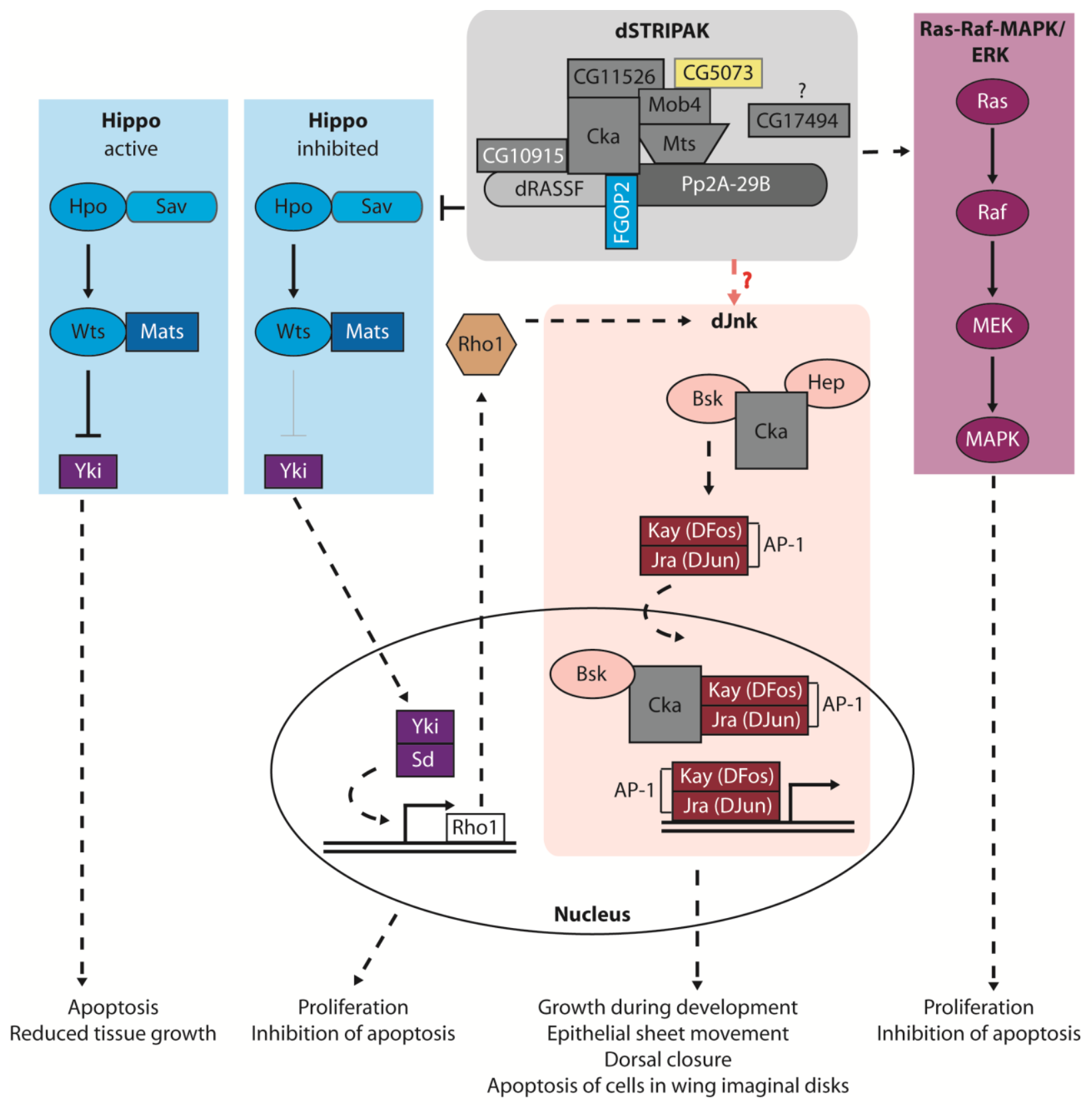

Figure 5: The dSTRIPAK inhibits Hippo signaling in D. melanogaster

Graphical representation of dSTRIPAK interaction with Hippo, dJnk and Ras-Raf-MAPK/ERK signaling. In the hippo pathway active Hpo kinase inhibits the transcription activator Yorkie (Yki). Cka and FGOP2 components of the dSTRIPAK negatively regulate hippo signaling during development. Yki translocates to the nucleus and together with Sd promotes rho1 transcription. Rho1 activates dJnk signaling. Cka associates with the kinases Hep and Bsk and brings the transcription factor AP1, composed of Kay (D-Fos) and Jra (DJun), into the nucleus. AP1 promotes transcription of target genes to ensure dorsal closure and regulates apoptosis in wing imaginal disks. It is unclear whether $\mathrm{Cka}$ acts together or independently of the dSTRIPAK complex to regulate dJnk signaling positively. Cka was also shown to activate Ras-Raf-MAPK/ERK signaling. Ras-Raf-MAPK/ERK signaling regulates cell growth, differentiation, proliferation, inhibition apoptosis and cell migration. Furthermore, Cka, the SLMAP ortholog CG17494, FGOP2, the STRIP ortholog CG11526 and Mob4 were identified to act upstream of Raf. Some parts of the figure are modified according to Zhao et al. (2011), Frey et al. (2015b), Ríos-Barrera and Riesgo-Escovar (2013) and Ashton-Beaucage et al. (2014). 
The Hippo pathway is a kinase cascade very similar to the yeast SIN/MEN pathways and controls organ size by suppressing cell proliferation (Hergovich \& Hemmings, 2012). The serine/threonine

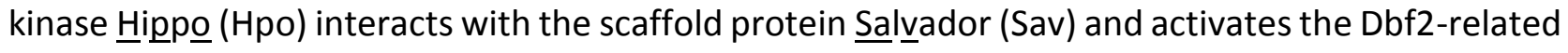

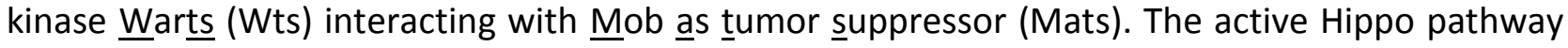
results in phosphorylation and inhibition of the transcriptional activator Yorkie (Yki). In contrast, Yki is dephosphorylated and active when the Hpo pathway is inhibited. Dephosphorylated Yki enters the nucleus, interacts with the transcription factor Scalloped (Sd), activates target genes to promote cell growth, and inhibits apoptosis (Huang et al., 2005b, Kango-Singh \& Singh, 2009, Lai et al., 2005, Tapon et al., 2002) (Figure 5).

Unfortunately, is not clear if the Hippo pathway in D. melanogaster controls mitotic exit and cytokinesis.

Taken together, the dSTRIPAK prevents Hpo activation promoting growth during development. However, the role of the dSTRIPAK in actin polymerization, mitotic exit and cytokinesis in D. melanogaster remains to be examined.

\subsubsection{Cka in the Jun-N-terminal kinase pathway in D. melanogaster}

The striatin ortholog Cka was identified as positive regulator of the Jun-N-terminal kinase (dJnk) pathway. Cka is required in epithelial sheet movement during embryonic dorsal closure and at later developmental stages to regulate apoptosis in wing imaginal wings (Chen et al., 2002).

Loss of function of the Ste20-related kinase, Misshapen (Msn) leads to a dorsal open phenotype in D. melanogaster and is similar to embryos lacking dJnk components. Msn, the ortholog of mammalian MINK1 kinase, was shown to function upstream of Hemipterous kinase (Hep), which is part of the dJnk signaling pathway (Su et al., 1998). Cka associates with the serine/threonine kinases Hep and Basket (Bsk). Jun-related antigen (Jra (D-Jun)) and Kayak (Kay (D-Fos)) dimerize to form the transcription factor AP1. Finally, Cka together with Bsk and AP1 translocate to the nucleus (Figure 5). Mutations in kay, jra and cka genes cause a dorsal open phenotype. Likewise, Jra and Cka control epithelial sheet movement during dorsal closure trough AP-1 mediated transcription (Chen et al., 2002). 
However, no other dSTRIPAK proteins than Cka were shown to interact with kinases of the dJnk pathway, thus, it is possible that the dSTRIPAK complex negatively regulates Hippo signaling but Cka acts in a dSTRIPAK-independent way with the dJnk pathway. It is also worth to think about the dSTRIPAK complex both as hippo signaling regulator and as courier to deliver Cka for interaction with the kinases Bsk and Hep.

In D. melanogaster, rho1 was identified as Yki/Sd target. Again, the dSTRIPAK was described to inhibit Hippo signaling (Ribeiro et al., 2010) and the small GTPase dRho1 activates dJnk when Hippo signaling is lost (Ma et al., 2015). Interestingly, the Far complex in S. cerevisiae was also connected to Rho-signaling. Far is suggested to regulate Rho-CWI signaling in, which Rho1 activates Pkc1 in the cell wall integrity pathway (Levin, 2005). Similar, dSTRIPAK might modulate CWI pathway in D. melanogaster via Yki-Sd-dRho1. Consistent with the results of Ma et. al., (2015) deletion of dSTRIPAK components renders Hippo signaling active, which suppresses dJnK activity via Yki1, Sd and dRho1 and results in suppression of the hpo overgrowth phenotype (Ribeiro et al., 2010) (Figure 5).

In sum, Cka is a component of the dSTRIPAK and associates with the protein phosphatase Pp2A29B and the SLMAP ortholog CG17494. D. melanogaster STRIPAK regulates growth during development, dorsal closure and apoptosis in wing imaginal disks and was connected to the Hippo and dJnk signaling pathways.

\subsubsection{The dSTRIPAK activates the Ras-Raf-MAPK/ERK pathway}

In addition to its involvement in Hippo signaling and the dJnk pathway, the dSTRIPAK acts as positive regulator of Ras-Raf-MAPK/ERK signaling. The Ras-Raf-MAPK/ERK signaling regulates cell growth, differentiation, migration and proliferation as well as inhibition of apoptosis (McCubrey et al., 2007, Santarpia et al., 2012) (Figure 5). Cka was shown to activate Ras-Raf-MAPK/ERK signaling and the dSTRIPAK components Cka, GCKIII and Mts were shown to associate with Raf (Friedman \& Perrimon, 2006, Horn et al., 2011). The five dSTRIPAK components, Cka, the SLMAP ortholog CG17494, FGOP2, the STRIP ortholog CG11526 and Mob4 act upstream of Raf positively regulating Raf (Ashton-Beaucage et al., 2014). In the same study, the authors supposed, that the dSTRIPAK 
Introduction

might facilitate PP2A binding to Raf. Unfortunately, the authors did not further investigate proteinprotein interactions between dSTRIPAK components and Ras-Raf-MAPK/ERK members.

Taken together, dSTRIPAK in D. melanogaster might positively regulate cell growth, differentiation, migration and proliferation as well as inhibition of apoptosis. However, it is not known if like in mammals mutually exclusive dSTRIPAK complexes exist in the fruit fly, which might regulate different signaling pathways.

\subsection{Sordaria macrospora is a model organism to study fruiting-body development}

This work concentrates on the STRIPAK complex in the eukaryote and multicellular filamentous fungus S. macrospora. Next, the model organism S. macrospora will be presented followed by the current knowledge about the STRIPAK complex in S. macrospora and other filamentous fungi.

Eukaryotic cell biology is studied in various lower eukaryotic model organisms for example yeasts or filamentous fungi. The letter are closer related to mammals than to plants making them interesting models to study molecular mechanisms also found in human cells (Pace, 2009, Van der Klei \& Veenhuis, 2006). S. macrospora is a filamentous fungus of the phylum Ascomycota, which is naturally found in the dung of herbivores (Esser, 1982). Transformation of mycelium to pear shaped fruiting bodies with various cell types requires complex and tight spatial-temporal regulation of the involved genes (Pöggeler et al., 2006, Teichert et al., 2014). During sexual development 13 different cell types are generated, which are not present in the vegetative mycelium (Lord \& Read, 2011). S. macrospora is a haploid and self-fertile filamentous fungus and therefore does not need a mating partner to propagate sexually by meiotic ascospores (Lord \& Read, 2011, Pöggeler et al., 1997). Thus, recessive mutations of genes that are involved in fruiting-body formation and differentiation can be directly detected. Hence, S. macrospora is an excellent model for genetic and microscopic analysis (Lord \& Read, 2011, Teichert et al., 2014). Many molecular and genetic methods are well established and the relatively small genome of 40 MB is fully sequenced (Nowrousian et al., 2012). Moreover, S. macrospora is not harmful, easy to manipulate and easy to grow under laboratory conditions (Kück et al., 2009).

The life cycle of S. macrospora in the laboratory takes 7 days. Sexual development starts at day 2 when the first sexual structures, the ascogonia, are formed. Ascogonia develop into 
protoperithecia at day 3-4 and into mature perithecia filled with ascospores at day 6-7 (Teichert et al., 2014) (Figure 6). In the tip of ascogonous hyphae, which emerge from ascogonia two nuclei pair up. These hyphae with two nuclei from a hook-like structure called the crozier. Subsequently, synchronous mitotic division and formation of two septa takes place. The lateral and the basal cell contain one nucleus each whereas the apical cell contains two nuclei, which fuse (karyogamy) followed by meiosis and a post-meiotic mitosis. Finally, the perithecia contain 50-300 asci each containing 8 linearly ordered ascospores, which are discharged trough the ostiolum, an apical pore in the perithecium. The ascospores germinate and the life cycle starts again (Engh et al., 2010, Kück et al., 2009, Esser, 1982) (Figure 6).

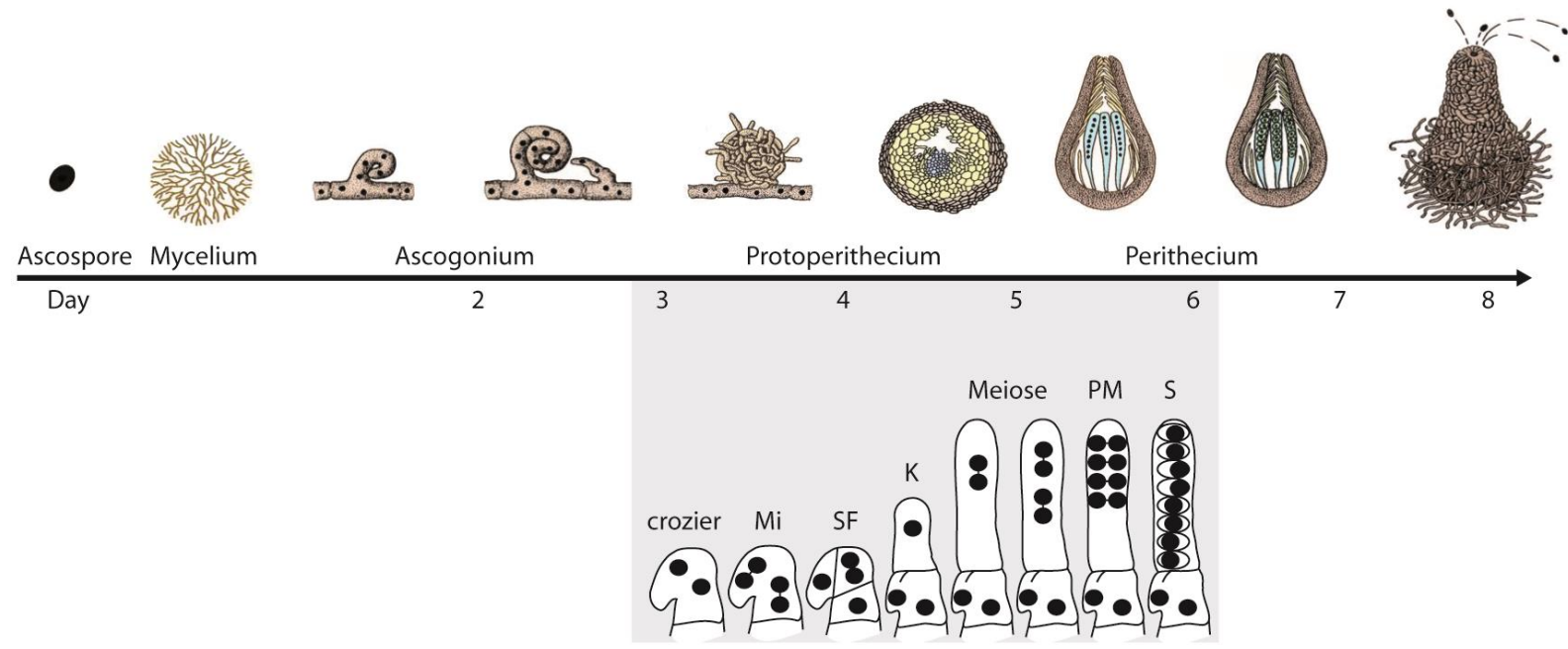

Figure 6: The life cycle of $S$. macrospora

Schematic representation of the $S$. macrospora life cycle in a time line. The life cycle starts with a germinating ascospore generating mycelium. From the mycelium, first sexual structures, the ascogonia, are formed at day 2, which develop into pre-fruiting bodies the protoperithecia at day 3-4. In ascogonous hyphae nuclei pair up followed by mitotic (Mi) and synchronous division followed by septum formation (SF), karyogamy (K) meiosis and postmeiotic mitosis (PM). Mature fruiting bodies (perithecia) contain 50-300 asci each with 8 linearly ordered ascospores $(\mathrm{S})$, which are discharged trough the ostiolum at day 7-8. Modified according to Pöggeler (2000), drawing modified according to H. J. Rathke.

\subsubsection{The STRIPAK complex in S. macrospora regulates sexual development and hyphal fusion}

The filamentous ascomycete S. macrospora propagates only via sexually formed fruiting bodies.

The process from a two-dimensional mycelium to three-dimensional fruiting bodies with various cell types requires complex and tight regulation. In the past, many factors needed for the development of fruiting bodies were identified (Kück et al., 2009, Teichert et al., 2014). In 
S. macrospora, one multi protein complex involved in this process is the striatin-interacting phosphatase and kinase complex (SmSTRIPAK) (Kück et al., 2016). This complex is of general interest beyond fungal development, since its components are conserved in fungi and animals (Bloemendal et al., 2012, Bloemendal et al., 2010, Engh et al., 2010, Goudreault et al., 2009, Nordzieke et al., 2015, Pöggeler \& Kück, 2004, Kück et al., 2016). In S. macrospora, the scaffolding subunit SmPP2AA of the serine/threonine phosphatase PP2A, interacted in yeast two-hybrid experiments with the striatin ortholog/B"' regulatory subunit protoperithecium mutant 11 (PRO11). Further, SmSTRIPAK core components are the catalytic PP2A subunit, SmPP2Ac1, the STRIP1/2 ortholog PRO22, the SLMAP ortholog PRO45, and the kinase activator SmMOB3 (Bernhards \& Pöggeler, 2011, Bloemendal et al., 2012, Bloemendal et al., 2010, Nordzieke et al., 2015, Beier et al., 2016). Additionally, the dual-targeted protein glycosylphosphatidylinositolanchored protein 1 (SmGPI1) and the GCKIII kinases SmKIN3 and SmKIN24 were identified to interact with STRIPAK components of S. macrospora (Frey et al., 2015a, Frey et al., 2015b).

\subsubsection{Striatin orthologs in filamentous fungi control hyphal fusion, sexual development and vegetative growth}

PRO11 is a protein with a predicted molecular mass of $90 \mathrm{kDa}$. PRO11 is suggested to provide a scaffold for the association of STRIPAK complex proteins (Bloemendal et al., 2012). Like all striatins, PRO11 is characterized by a $\mathrm{Ca}^{2+}$-calmodulin-binding site, a CC domain, a putative caveolin-binding site and 7 WD40 repeats (Pöggeler \& Kück, 2004). However, caveolin orthologs are not encoded by S. macrospora or other filamentous fungi (Nowrousian, 2010, Pöggeler \& Kück, 2004).

A nonsense mutation in the pro11 gene with a STOP codon at the amino-acid position 546 results in a truncated PRO11 protein lacking several WD40 repeats (Pöggeler \& Kück, 2004). The corresponding pro11 strain develops protoperithecia, but is not able to form mature fruiting bodies (perithecia). Likewise, mutation or deletion of pro11 results in a sterile phenotype, which is devoid of hyphal fusion. Interestingly, striatins are functionally conserved because mouse striatin cDNA complemented the sterile phenotype of pro11 (Pöggeler \& Kück, 2004).

The hyphal anastomosis protein 3 (HAM-3) is part of the STRIPAK complex in the closely related fungus Neurospora crassa controlling hyphal fusion and sexual development (Simonin et al., 2010). The Aspergillus nidulans striatin ortholog StrA was reported to be important for colony growth, 
cleistothecium development and ascosporogenesis (Wang et al., 2010). A striatin ortholog, which is involved in virulence and sexual mating is also found in Fusarium verticillioides and in Fusarium graminearum (Shim et al., 2006). The deletion of the striatin orthologous gene str1 in Colletotrichum graminicola results in a defect in hyphal fusion and virulence (Wang et al., 2016).

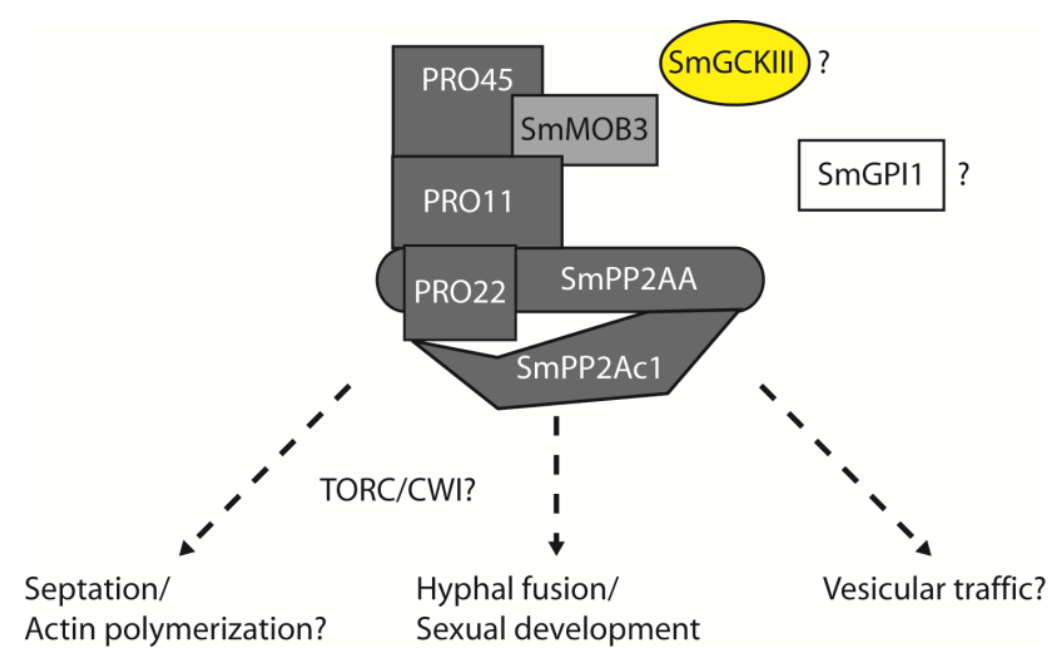

Figure 7: The STRIPAK complex in S. macrospora

The core STRIPAK complex in S. macrospora includes the striatin ortholog PRO11, the STRIP $1 / 2$ ortholog PRO22, the MOB3 (phocein) ortholog SMMOB3, the SLMAP ortholog PRO45, and the protein phosphatase 2 A scaffolding and catalytic subunit SmPP2AA and SmPP2Ac1, respectively. STRIPAK-associated proteins in S. macrospora are the dual-targeted protein SmGPI1 and the GCKIII kinases SmKIN3 and SmKIN24. The STRIPAK is involved in septation, was connected to TORC signaling and to the CWI pathway, through SmPP2Ac1 and PRO22 but underlying molecular processes are unknown. Involvement of SmMOB3 in vesicle transport is suggested but was not experimentally verified. Modified according to Bloemendal et al. (2012), Beier et al. (2016) and Nordzieke et al. (2015).

\subsubsection{Fungal MOB3 proteins are involved in hyphal fusion, development and vesicular traffic}

In S. macrospora, the MOB3 (phocein) ortholog SmMOB3 has N-terminal and C-terminal stretches and thus is three times longer compared to MOB3 (phocein) proteins in animals. SmMOB3 contains a conserved $\mathrm{MOB}$ domain and has a predicted molecular mass of $72 \mathrm{kDa}$. Fungal MOB3 proteins also contain a region, which is homologous to the small chain signature of the $\sigma$ subunits of clathrin adaptor (AP) complexes and a region homologous to a PxxDY type SH-binding domain (Baillat et al., 2001, Bernhards \& Pöggeler, 2011). In general, proteins with these domains are associated with endocytosis (Musacchio et al., 1992, Popova et al., 2013, Verschueren et al., 2015). In mammals, 
MOB3 (phocein) associates with NDPK, Eps15 and dynamin and was suggested to be involved in vesicular traffic and clathrin-mediated endocytosis (Baillat et al., 2002, Baillat et al., 2001).

In S. macrospora, SmMOB3 interacts with the striatin ortholog PRO11 and is involved in hyphal fusion and sexual development (Bernhards \& Pöggeler, 2011). The in silico analysis of the SmMOB3 protein indicated that the SMSTRIPAK might be linked to vesicular traffic and endocytosis (Bernhards \& Pöggeler, 2011, Bloemendal et al., 2012). The MOB-3 protein in N. crassa was also reported to be involved in hyphal fusion and sexual development (Maerz et al., 2009). In Epichloë festucae, the MOB3 ortholog MobC is required to maintain the mutualistic symbiotic interaction between the fungus and the cool-season grass Lolium perenne and regulates cell-cell fusion and expressoria formation (Green et al., 2016).

\subsubsection{The fungal STRIP1/2 ortholog and the catalytic PP2ac1 subunit link the fungal STRIPAK to septation and the CWI pathway}

Further STRIPAK components were identified in S. macrospora. The PRO11 interaction partner PRO22 (STRIP1/2) is involved in septum formation in ascogonia as well as associates with tubular vacuoles (Bloemendal et al., 2010). PRO22 is a protein with a calculated size of $124 \mathrm{kDa}$ containing three predicted transmembrane domains (Bloemendal et al., 2010). In N. crassa the STRIP 1/2 ortholog hyphal anastomosis-2 (HAM-2) was found to be a transmembrane protein required for hyphal fusion and sexual development. Deletion of the ham-2 gene leads to female sterility, slow growth rate, lack of hyphal fusion, and shortened areal hyphae (Xiang et al., 2002). Roca et al. (2005) showed that the ham-2 gene is required for fusion of conidial anastomosis tubes (CATs) and thus for fusion of conidial germlings. In fluorescence microscopy HAM-2 was localized to the nuclear envelope in N. crassa (Dettmann et al., 2013).

Recently, in S. macrospora the catalytic PP2A subunit SmPP2Ac1 was identified in a tandem affinity purification coupled with mass spectrometry approach (TAP-MS) using PRO22 as bait (Bloemendal et al., 2012). SmPP2Ac1 is required for phosphatase activity, sexual development and septum formation in ascogonia. Septation was missing in early reproductive structures in Smpp2ac1 and also in pro22 deletion mutants (Beier et al., 2016, Bloemendal et al., 2010). In the same study STRIPAK components were connected to TORC signaling and to the cell wall integrity MAP kinase pathway (Beier et al., 2016). Consistently, the studies of Dettmann et al. (2013) and Fu et al. (2011) 
Introduction

suggested interactions between the STRIPAK and the cell wall integrity MAP kinase pathway in N. crassa.

\subsubsection{The SLMAP ortholog PRO45 is part of the fungal STRIPAK complex in S. macrospora}

The SLMAP ortholog PRO45 was demonstrated to interact with PRO11 and SmMOB3. PRO45 has a predicted size of $86 \mathrm{kDa}$ and contains a C-terminal transmembrane (TM) domain, a forkheadassociated (FHA) domain in the N-terminal part and two CC regions between the FHA and the TM domain. PRO45 associates with the endoplasmic reticulum (ER), the nuclear envelope and mitochondria (Nordzieke et al., 2015). Fluorescence microscopy showed that the SLMAP ortholog HAM-4 localized to the nuclear envelope in a patchy manner and to the endomembrane system. In cell fractionation assays HAM-4 was present in the nuclear fraction and a minor amount was present in the cytosol/endomembrane fraction (Dettmann et al., 2013). In N. crassa, the striatin ortholog HAM-3 and the tail-anchored protein HAM-4 were shown to function together in the NcSTRIPAK complex and influence hyphal fusion and sexual development (Simonin et al., 2010). Simonin et al. (2010) showed that ham-2, ham-3 and ham-4 mutants are reduced in growth rate, have short areal hyphae and an aberrant ascospore development.

\subsubsection{Fungal STRIPAK-associated proteins SmGPI1 and GCKIII kinases SmKIN3 and SmKIN24}

Interaction between the kinase activator SmMOB3 and the glycosylphosphatidylinositol-anchored protein 1 (SmGPI1) was shown in S. macrospora. SmGPI1 is a protein with a calculated molecular mass of $26 \mathrm{kDa}$ containing a secretion signal sequence in the $\mathrm{N}$-terminal part of the protein and a region for GPI-anchor attachment in the C-terminal part of the protein. SmGPI1 is suggested to be a negative regulator of sexual development and localizes to the plasma membrane, cell wall and to mitochondria (Frey et al., 2015a).

The GCKIII kinases SmKIN3 and SmKIN24 were shown to physically interact with PRO11 and might link the SmSTRIPAK complex to a kinase-dependent signaling pathway. SmKIN3 and SmKIN24 have a predicted molecular mass of 74 and $98 \mathrm{kDa}$, respectively. The catalytic domain of SmKIN3 and SmKIN24 are in the N-terminal regions of the proteins and shows the highest homology to Ste20like kinase3 serine/threonine-protein kinases. Deletion of SmKIN3 and SmKIN24 results in a septation phenotype in young vegetative hyphae (Frey et al., 2015b). Interestingly, the 
Introduction

developmental protein PRO22 and the catalytic PP2A subunit SmPP2Ac1 are also connected to septation in S. macrospora (Beier et al., 2016, Bloemendal et al., 2010). However, the connection between subcellular location and the mode of action of STRIPAK signaling in S. macrospora is unclear.

\section{All in all, orthologs of the STRIPAK that are found in higher eukaryotes are also present in the fungal STRIPAK complex controlling fungal sexual development, hyphal fusion and vegetative growth.}

\subsection{Aims of this thesis}

Filamentous fungi undergo multicellular differentiation during their life cycle and are excellent models to study developmental processes like fruiting-body development (Pöggeler et al., 2006, Teichert et al., 2014).

S. macrospora is a non-pathogenic fungus generating three-dimensional fruiting bodies from two dimensional mycelium within 7 days in the laboratory (Pöggeler et al., 2006, Teichert et al., 2014). The fact that S. macrospora is homothallic, and self-fertile facilitates detection of recessive mutations (Lord \& Read, 2011, Pöggeler et al., 1997). Mutants defective in sexual development were generated by UV light and ethyl methanesulfonate mutagenesis (Masloff et al., 1999, Pöggeler \& Kück, 2004). Among them, three mutants pro11, pro22 and pro45 defective in STRIPAK function were detected (Bloemendal et al., 2010, Nordzieke et al., 2015, Pöggeler \& Kück, 2004). The STRIPAK complex is conserved in animals and fungi and fundamental developmental processes are governed by the STRIPAK complex. In yeasts its function is required for cytokinesis, septum formation, actin polymerization, autophagy and mitotic exit (Baryshnikova et al., 2010, Gupta \& McCollum, 2011, Pracheil \& Liu, 2013, Singh et al., 2011). In mammals, STRIPAK complex is required for normal function of neuronal cells and neuronal development, cell to cell adhesion, cytoskeleton organization and vesicular transport (Bai et al., 2011, Baillat et al., 2001, Bartoli et al., 1999, Breitman et al., 2008, Castets et al., 1996). STRIPAK dysfunction was connected to neuronal and cardiac disease and to cancer (Bartoli et al., 1999, Chen \& Hsueh, 2012, Grand et al., 2004, Hwang \& Pallas, 2014, Ishikawa et al., 2012, Lin et al., 2010, Meurs et al., 2010). 
The STRIPAK complex in filamentous fungi is required during development and for virulence but STRIPAK signaling in filamentous fungi is poorly investigated (Beier et al., 2016, Bernhards \& Pöggeler, 2011, Bloemendal et al., 2012, Bloemendal et al., 2010, Fu et al., 2011, Green et al., 2016, Islam et al., 2017, Nordzieke et al., 2015, Pöggeler \& Kück, 2004, Shim et al., 2006, Simonin et al., 2010, Wang et al., 2010, Wang et al., 2016, Zhang et al., 2017).

In this study, pull-downs of the central SmSTRIPAK component PRO11 (striatin) and its strong interaction partner SmMOB3 coupled to LC/MS were used to search for possible interacting partners. The aim was to identify and functionally analyze potential interaction partners of PRO11 and SmMOB3 in S. macrospora. An unstudied potential interaction partner, named SCl1 was identified with very high confidence. It's conservation among yeasts, filamentous fungi, the fruit fly and mammals should be investigated by phylogenetic analysis. As the SmSTRIPAK is involved in development, qRT-PCR should be performed to access the regulation of the unstudied gene during sexual development. A sci1 deletion mutant should be generated to investigate the role of the unstudied gene in vegetative growth, hyphal fusion and sexual development. To investigate direct interaction of the SCI1 protein with other known SmSTRIPAK components $\mathrm{Y} 2 \mathrm{H}$ analysis should be performed. Also, pull-downs coupled to LC/MS with C-terminally eGFP-tagged SCI1 should assess, which proteins and STRIPAK proteins can be enriched. Mammalian and yeast orthologs of the identified protein should be transformed into the newly generated deletion mutant to investigate a possibly conserved function among phyla. Fluorescence microscopy studies will visualize the localization of the eGFP-tagged PRO11 and eGFP or DsRED-tagged SCI1. The STRIPAK in fungi, was connected to ER, mitochondria function, actin polymerization and vesicular traffic (Baryshnikova et al., 2010, Beier et al., 2016, Bernhards \& Pöggeler, 2011, Bloemendal et al., 2010, Frey et al., 2015a, Nordzieke et al., 2015, Pracheil et al., 2012). Therefore, morphology of nuclei, mitochondria and ER investigated by fluorescence microscopy as well as actin polymerization and endosome transport will help to shed light on the cellular function of SCl1. 


\section{Materials and Methods}

\section{$1.5 \quad$ Materials}

\subsubsection{Strains}

Table 2: Strains generated and used in this study

\begin{tabular}{|c|c|c|}
\hline Strain & Genotype & Source \\
\hline \multicolumn{3}{|l|}{ Escherichia coli } \\
\hline Mach1 $1^{\mathrm{TM}}$ & $\begin{array}{l}\begin{array}{l}\Delta r e c A 1398 \\
\left.m K^{+}\right) \mathrm{F}^{-}\end{array}\end{array}$ & Invitrogen, USA \\
\hline $\begin{array}{l}\text { StrataClone } \\
\text { SoloPack } \\
\text { competent cells }\end{array}$ & recA endA1 $\Delta$ tonA $F^{-}$lacZ $\Delta M 15 r_{K}^{-} S t^{R}$ & $\begin{array}{l}\text { Stratagen, USA and } \\
\text { Kanada }\end{array}$ \\
\hline $\mathrm{DH} 5 \alpha^{\mathrm{TM}}$ & 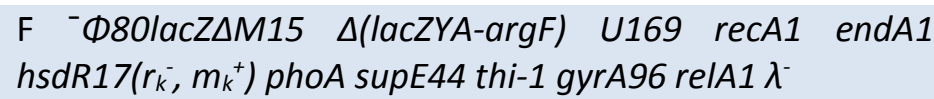 & Invitrogen, USA \\
\hline
\end{tabular}

\section{Saccharomyces cerevisiae}

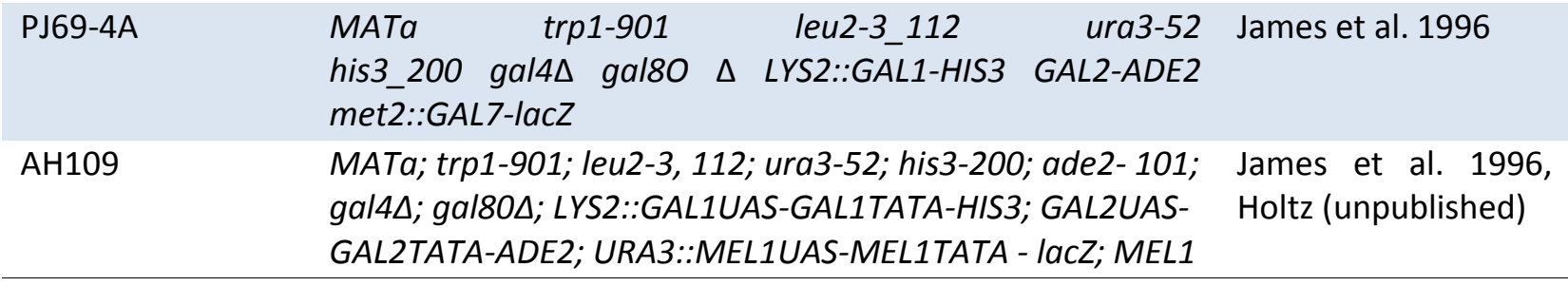

\section{Sordaria macrospora}

\begin{tabular}{|c|c|c|}
\hline wt (S48977) & wild type, black ascospores & Culture collection $^{a}$ \\
\hline fus1-1 (84595) & mutation in fus1-1 gene, brown ascospores & $\begin{array}{l}\text { Nowrousian et al. } \\
\text { (2012) }\end{array}$ \\
\hline$\Delta \mathrm{ku} 70$ (S66001) & $\begin{array}{l}\text { nat }^{R}, \text { ssi, fertile } \\
\Delta k u 70:: n a t\end{array}$ & $\begin{array}{l}\text { Pöggeler and Kück } \\
\text { (2006) }\end{array}$ \\
\hline$\Delta$ ku80::hph & $\begin{array}{l}h y g^{R}, \text { ssi, fertile } \\
\Delta k u 80:: h p h\end{array}$ & $\begin{array}{l}\text { Herzog and Pöggeler } \\
\text { (unpublished) }\end{array}$ \\
\hline$\Delta$ pro11 (S5.7) & $\begin{array}{l}h_{y g^{R}}, \text { ssi, sterile } \\
\Delta p r o 11:: h p h^{R}\end{array}$ & $\begin{array}{l}\text { Bloemendal et al. } \\
\text { (2012) }\end{array}$ \\
\hline
\end{tabular}




\begin{tabular}{|c|c|c|}
\hline$\Delta$ pro11/fus1 & $\begin{array}{l}h y g^{R}, \text { brown ssi, sterile } \\
\Delta p r o 11:: h p h^{R}\end{array}$ & Bernhards (2010) \\
\hline$\Delta$ Smmob3 & $\begin{array}{l}h y g^{R}, \text { ssi, sterile } \\
\Delta S m m o b 3:: h p h^{R}\end{array}$ & $\begin{array}{ll}\text { Bernhards } & \text { and } \\
\text { Pöggeler (2011) }\end{array}$ \\
\hline$\Delta$ Smmob3/fus1 & $\begin{array}{l}h y g^{R}, \text { brown ssi, sterile } \\
\Delta S m m o b 3:: h p h^{R}\end{array}$ & $\begin{array}{ll}\text { Bernhards } & \text { and } \\
\text { Pöggeler (2011) } & \end{array}$ \\
\hline$\Delta$ sci $_{\text {hyg }}$ & $\begin{array}{l}h y g^{R}, \text { ssi, sterile } \\
\Delta s c i 1:: h p h^{R}\end{array}$ & This study \\
\hline fus $\Delta$ sci $1_{\text {hyg }}$ & $\begin{array}{l}h y g^{R}, \text { brown ssi, sterile } \\
\Delta s c i 1:: h p h^{R}\end{array}$ & This study \\
\hline$\Delta$ sci $_{\text {nat }}$ & $\begin{array}{l}\text { nat }^{R} \text {, ssi, sterile } \\
\Delta \text { sci1::nat }\end{array}$ & This study \\
\hline$\Delta$ sci1 ${ }_{\text {hyg }} \Delta$ pro11 & $\begin{array}{l}h y g^{R}, \text { ssi, sterile } \\
\Delta s c i 1:: h p h^{R} \text { and } \Delta p r o 11:: h p h^{R}\end{array}$ & This study \\
\hline
\end{tabular}

\section{Pull-down and Localization}

\begin{tabular}{|c|c|c|}
\hline $\begin{array}{l}\text { Apro11::hapro11 } \\
\text { (TGS11) }\end{array}$ & $\begin{array}{l}\text { ectopic integration of pHA11 into } \Delta \text { pro11; } n a t^{R}, h y g^{R}, p T \text {, } \\
\text { fertile, Pccg1::ha::pro11::TtrpC }\end{array}$ & $\begin{array}{l}\text { Bloemendal et al. } \\
\text { (2012) }\end{array}$ \\
\hline $\begin{array}{l}\text { pro11:: } \\
\text { hapro11egfp }\end{array}$ & $\begin{array}{l}\text { ectopic integration of pHAPRO11GFP into pro11; } \text { at }^{\mathrm{R}}, \mathrm{pT} \text {, } \\
\text { fertile, Pccg1::ha::pro11::egfp::TtrpC }\end{array}$ & $\begin{array}{l}\text { Herzog and Pöggeler } \\
\text { (unpublished) }\end{array}$ \\
\hline $\begin{array}{l}\Delta \text { Smmob3_gpd- } \\
\text { MOB3:GFP }\end{array}$ & $\begin{array}{l}\text { ectopic integration of pMob3GFP into } \Delta \text { SmMOB3; } \text { nat }^{R} \text {, } \\
h_{y g^{R}}, \mathrm{pT} \text {, fertile, Pgpd::Smmob3::egfp::TtrpC }\end{array}$ & $\begin{array}{l}\text { Bernhards and } \\
\text { Pöggeler (2011) }\end{array}$ \\
\hline $\begin{array}{l}\text { fus::flagSmmob3 } \\
\text { (TGSM3) }\end{array}$ & $\begin{array}{l}\text { ectopic integration of pFLAGMob3 into S84595; } h y g^{R}, \mathrm{pT} \text {, } \\
\text { fertile, Pccg1::flag::Smmob3::TtrpC }\end{array}$ & $\begin{array}{l}\text { Bloemendal et al. } \\
\text { (2012) }\end{array}$ \\
\hline$\Delta$ sci1 hyg::scilegfp & $\begin{array}{l}\text { ectopic integration of psci1gfp into } \Delta s c i 1 ; n a t^{R}, h y g^{R}, \text { pT, } \\
\text { fertile, Pccg1::sci1::egfp::TtrpC }\end{array}$ & This study \\
\hline wt::ha & $\begin{array}{l}\text { ectopic integration of pHA_nat into } \$ 48977 \text {; } n a t^{R} \text {, ssi, fertile } \\
\text { Pccg1::ha::TtrpC }\end{array}$ & This study \\
\hline wt::flag & $\begin{array}{l}\text { ectopic integration of pFLAG_nat into S48977; nat }{ }^{R} \text {, ssi, } \\
\text { fertile } \\
\text { Pccg1::flag::TtrpC }\end{array}$ & This study \\
\hline wt::egfp & $\begin{array}{l}\text { ectopic integration of p1783-1 into } 548977 ; h g^{R} \text {, ssi, fertile } \\
\text { Pgpd::egfp::TtrpC }\end{array}$ & $\begin{array}{l}\text { Voigt and Pöggeler } \\
\text { (2013) }\end{array}$ \\
\hline
\end{tabular}




\section{Complementation}

\begin{tabular}{|c|c|c|}
\hline $\begin{array}{l}\Delta \text { sci1 hyg:: } \\
5^{\prime} \text { sci1egfp }\end{array}$ & $\begin{array}{l}\text { ectopic integration of } \mathrm{p} 5^{\prime} \text { sci1gfp with } 5^{\prime} \text { flanking region of } \\
\text { sci1 into } \Delta \text { sci }_{\text {hyg; }} \text { nat }{ }^{R}, \quad \text { hyg }^{R}, \quad \text { pT, fertile, } \\
\text { 5'sci1::sci1::egfp::TtrpC }\end{array}$ & This study \\
\hline $\begin{array}{l}\text { fus } \Delta \text { sci } 1_{\text {hyg }}: \\
5^{\prime} \text { sci1egfp }\end{array}$ & $\begin{array}{l}\text { ectopic integration of } 5^{\prime} \text { sci1gfp with } 5^{\prime} \text { flanking region of } \\
\text { sci1 into fus } \Delta \text { sci1 } 1_{\text {hyg }} ; \quad \text { nat }{ }^{R}, \quad h y g^{R}, \quad \text { pT, fertile, } \\
5^{\prime} \text { sci1::sci1::egfp::TtrpC }\end{array}$ & This study \\
\hline$\Delta$ sci $_{\text {hyg }}::$ sci1 $1_{63-207}$ & $\begin{array}{l}\text { ectopic integration of psci1 } 1_{63-207} \text { nat with } 5^{\prime} \text { flanking region } \\
\text { of sci1 into } \Delta \text { sci1 }_{\text {hyg; }} \text { nat }{ }^{R}, \text { hyg }^{R}, \mathrm{pT} \text {, reduced fertility, } \\
\text { 5'sci1::sci1(AA63-207)::egfp::TtrpC }\end{array}$ & This study \\
\hline$\Delta$ sci1 $_{\text {hyg }}::$ sci $1_{121-207}$ & $\begin{array}{l}\text { ectopic integration of psci1 } 1_{121-207} \text { nat with } 5^{\prime} \text { flanking } \\
\text { region of sci1 into } \Delta \text { sci } 1_{\text {hyg; }} \text { nat }{ }^{R}, h_{y g^{R}}, \mathrm{pT} \text {, sterile, } \\
5^{\prime} \text { sci1::sci1(AA121-207)::egfp::TtrpC }\end{array}$ & This study \\
\hline$\Delta$ sci $_{\text {hyg }}:: s c i 1_{121-298}$ & 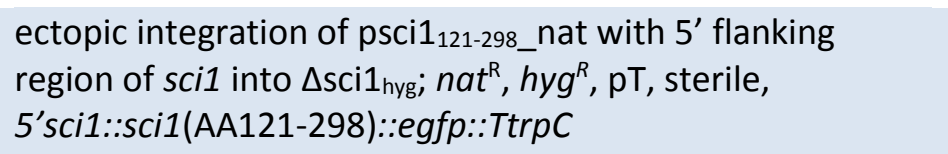 & This study \\
\hline$\Delta s c i 1_{\text {hyg }}:: s c i 1_{208-298}$ & $\begin{array}{l}\text { ectopic integration of psci1 } 1_{208-298} \text { nat with } 5^{\prime} \text { flanking } \\
\text { region of sci1 into } \Delta \text { sci1 } 1_{\text {hyg; }} \text { nat }{ }^{R}, h_{y g^{R}}, \mathrm{pT} \text {, sterile, } \\
\text { 5'sci1::sci1(AA208-298)::egfp::TtrpC }\end{array}$ & This study \\
\hline $\begin{array}{l}\Delta \text { sci1 }_{\text {hyg }:} \\
\text { 5'sci1Dsred }^{\prime}\end{array}$ & $\begin{array}{l}\text { ectopic integration of } \mathrm{p} 5 \text { 'sci1Dsred with } 5^{\prime} \text { flanking region } \\
\text { of sci1 into } \Delta \text { sci }_{\text {hyg; }} \text { nat }{ }^{R}, \quad h y g^{R}, \quad \text { pT, fertile, } \\
5^{\prime} \text { sci1::sci1::Dsred::TtrpC }\end{array}$ & This study \\
\hline $\begin{array}{l}\Delta \text { sci1 }_{\text {hyg }::} \\
\text { Rnfgfr1op2 }\end{array}$ & $\begin{array}{l}\text { ectopic integration of pPcFGwtRN into } \Delta s c i 1_{\text {hyg }} n a t^{R}, h y g^{R} \text {, } \\
\text { pT, sterile, Pccg1::Rnfgfr1op2::ha::TtrpC }\end{array}$ & This study \\
\hline $\begin{array}{l}\Delta s c i 1_{\text {hyg }}: \\
\text { Hsfgfr1op2 }\end{array}$ & $\begin{array}{l}\text { ectopic integration of pPcFGwtHS into } \Delta \text { sci }_{\text {hyg }} \text { nat }{ }^{R}, h y g^{R} \text {, } \\
\text { pT, sterile, Pccg1::HsFGFR1OP2::ha::TtrpC }\end{array}$ & This study \\
\hline $\begin{array}{l}\Delta s c i 1_{\text {hyg }::} \\
\text { Hsfgfr1op2SNP1 }\end{array}$ & $\begin{array}{l}\text { ectopic integration of pPcFGSNP1 into } \Delta \text { sci }_{\text {hyg }} \text { nat } \\
\text { pT, }{ }^{R} \text {, sterile, } \text { Pccg1 }{ }^{R} \text {,: HsFGFR1OP2SNP1::ha::TtrpC }\end{array}$ & This study \\
\hline $\begin{array}{l}\Delta \text { sci1 }_{\text {hyg }}: \\
\text { ScFAR3 }\end{array}$ & $\begin{array}{l}\text { ectopic integration of pFar3HA into } \Delta \mathrm{sci}_{\mathrm{hyg}} ; \text { nat }^{\mathrm{R}}, \text { hyg }^{R}, \mathrm{pT} \text {, } \\
\text { sterile, Pccg1::ScFAR3::ha::TtrpC }\end{array}$ & This study \\
\hline $\begin{array}{l}\Delta \text { sci } 1_{\text {hyg }}:: \\
\text { ScFAR7 }\end{array}$ & $\begin{array}{l}\text { ectopic integration of pFar7FLAG into } \Delta \text { sci1 }_{\text {hyg }} ; n a t^{R}, h y g^{R} \text {, } \\
\text { pT, sterile, Pccg1::ScFAR7::flag::TtrpC }\end{array}$ & This study \\
\hline
\end{tabular}

\section{Localization}

$\Delta$ sci1 $1_{\text {hyg }}:$ sci1Dsred ectopic integration of psci1Dsred into $\Delta s c i 1_{\text {hyg }} n a t^{R}, h y g^{R}$, This study pT, fertile, Pccg1::sci1::Dsred::TtrpC

$\Delta$ pro11::

hapro11egfp ectopic integration of pHAPRO11GFP into $\Delta$ pro11; $n a t^{R}$, This study $h y g^{R}$, pT, fertile, Pccg1::ha::pro11::egfp::TtrpC 


\begin{tabular}{|c|c|c|}
\hline $\begin{array}{l}\Delta \text { sci }_{\text {hyg }} \Delta \text { pro11:: } \\
\text { sci1Dsred+ } \\
\text { hapro11egfp }\end{array}$ & 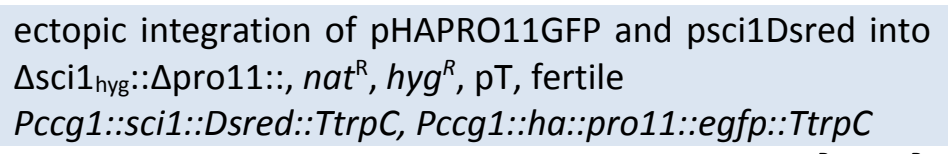 & This study \\
\hline $\begin{array}{l}\Delta s c i 1_{\text {hyg }::} \\
\text { h2btdTomato }\end{array}$ & $\begin{array}{l}\text { ectopic integration of pRH2B_nat into } \Delta \text { sci1 }_{\text {hyg }} ; n a t^{R}, h y g^{R} \text {, } \\
\text { pT, sterile }\end{array}$ & This study \\
\hline $\begin{array}{l}\text { wt:: } \\
\text { h2btdTomato }\end{array}$ & $\begin{array}{l}\text { ectopic integration of } \mathrm{pRH} 2 \mathrm{~B} \text { _nat into S48977; } n a t^{\mathrm{R}} \text {, pT, } \\
\text { fertile }\end{array}$ & This study \\
\hline $\begin{array}{l}\Delta s c i 1_{\text {hyg }}: \\
\text { mitoDsred }\end{array}$ & $\begin{array}{l}\text { ectopic integration of pMito-DsRed into } \Delta s c i 1_{\text {hyg }} n a t^{R}, h y g^{R} \text {, } \\
\text { pT, sterile }\end{array}$ & This study \\
\hline $\begin{array}{l}\text { wt:: } \\
\text { mitoDsred }\end{array}$ & $\begin{array}{l}\text { ectopic integration of pMito-DsRed into S48977; } \\
n a t^{R}, p T \text {, fertile }\end{array}$ & This study \\
\hline $\begin{array}{l}\Delta \text { sci1 }_{\text {hyg }::} \\
\text { Lifeact-tagrfp }\end{array}$ & $\begin{array}{l}\text { ectopic integration of pAL4-Lifeact into } \Delta \text { sci1 } 1_{\text {hyg }} \\
n a t^{R}, h y g^{R}, p T \text {, sterile }\end{array}$ & This study \\
\hline $\begin{array}{l}\text { wt:: } \\
\text { lifeact-tagrfp }\end{array}$ & $\begin{array}{l}\text { ectopic integration of pAL4-Lifeact into S48977; } \\
n a t^{R}, p T \text {, fertile }\end{array}$ & This study \\
\hline $\begin{array}{l}\Delta s c i 1_{\text {hyg }}: \\
\text { DsredKDEL }\end{array}$ & $\begin{array}{l}\text { ectopic integration of pDsREDKDEL into } \Delta \text { sci } 1_{\text {hyg; }} \\
n a t^{R}, h y g^{R}, p T \text {, sterile }\end{array}$ & This study \\
\hline $\begin{array}{l}\text { wt:: } \\
\text { DsredKDEL }\end{array}$ & $\begin{array}{l}\text { ectopic integration of pDsREDKDEL into S48977; } \\
n a t^{\mathrm{R}}, \mathrm{pT} \text {, fertile }\end{array}$ & This study \\
\hline $\begin{array}{l}\Delta \text { sci1 }_{\text {hyg }::} \\
\text { Smpom152egfp }\end{array}$ & $\begin{array}{l}\text { ectopic integration of pSmPOM152GFP into } \Delta \text { sci1 }{ }_{\text {hyg; }} \\
n a t^{R}, h y g^{R}, p T \text {, sterile }\end{array}$ & This study \\
\hline $\begin{array}{l}\text { wt:: } \\
\text { Smpom152egfp }\end{array}$ & $\begin{array}{l}\text { ectopic integration of of pSmPOM152GFP into S48977; } \\
n a t^{R}, p T \text {, fertile }\end{array}$ & This study \\
\hline $\begin{array}{l}\Delta \text { sci } 1_{\text {nat }}: \\
\text { egfpZtrab5 }\end{array}$ & $\begin{array}{l}\text { ectopic integration of pHEgfpRab5 into } \Delta \text { sci1 }_{\text {nat; }} \\
\Delta \text { sci1::nat }{ }^{R}, h y g^{R}, \text { pT, sterile }\end{array}$ & This study \\
\hline $\begin{array}{l}\text { wt:: } \\
\text { egfpZtrab5 }\end{array}$ & $\begin{array}{l}\text { ectopic integration of pHEgfpRab5 into S48977; } \\
h y g^{R}, \mathrm{pT} \text {, fertile }\end{array}$ & This study \\
\hline $\begin{array}{l}\Delta \text { sci } 1_{\text {nat }}: \\
\text { egfpZtrab7 }\end{array}$ & $\begin{array}{l}\text { ectopic integration of pHEgfpRab7 into } \Delta \text { sci1 }_{\text {nat; }} \\
\Delta \text { sci1::nat }{ }^{R}, h y g^{R}, \text { pT, sterile }\end{array}$ & This study \\
\hline $\begin{array}{l}\text { wt:: } \\
\text { egfpZtrab7 }\end{array}$ & $\begin{array}{l}\text { ectopic integration of pHEgfpRab7 into S48977; } \\
h y g^{R}, \mathrm{pT} \text {, fertile }\end{array}$ & This study \\
\hline $\begin{array}{l}\triangle \text { Smmob3_gpd- } \\
\text { MOB3:DsRED }\end{array}$ & $\begin{array}{l}\text { ectopic integration of pMob3DsRed into } \triangle \text { SmMOB3; } n a t^{R} \text {, } \\
h y g^{R}, \mathrm{pT} \text {, fertile, Pgpd::Smmob3::Dsred::TtrpC }\end{array}$ & Bernhards (2010) \\
\hline wt::Dsred & $\begin{array}{l}\text { ectopic integration of pRHN1 into } 548977 ; h g^{R} \text {, ssi, fertile } \\
\text { Pgpd::Dsred::TtrpC }\end{array}$ & Werner (2017) \\
\hline
\end{tabular}


The native promoter and terminator of sci1 are named 5'sci1 and 3'sci1 respectively. Other promoters and terminators are marked with a P or T respectively; ssi: single spore isolate, $\mathrm{pT}$ : primary transformant ${ }^{\mathrm{a}}$ Department for General and Molecular Botany, Ruhr-University Bochum

\subsubsection{Plasmids}

Table 3: List of plasmids used and generated in this study

\begin{tabular}{lll}
\hline Plasmid & Characteristics & Source \\
\hline Plasmid Backbones & & \\
pRS426 & $a m p^{R}$, URA3 & Christianson and \\
pRSnat & $a m p^{R}$, URA3, nat & Fierke (1996) \\
pRShyg & $a m p^{R}$, URA3, hph & Klix et al. (2010) \\
pFLAGN1 & $a m p^{R}$, his-3, & Bloemendal et al. \\
& Pccg-1::flag & (2012) \\
pHAN1 & $a m p^{R}$, his-3, & Kawabata and Inoue \\
& Pccg-1::ha & (2007) \\
pCB1003 & $a m p^{R}$, hph & Kawabata and Inoue \\
& & (2007) \\
\hline
\end{tabular}

\section{Deletion constructs}

\begin{tabular}{ll} 
pKOsci1hyg & $\begin{array}{l}a m p^{R}, U R A 3,899 \mathrm{bp} \text { of the } 5^{\prime} \text { flanking region and } 637 \mathrm{bp} \\
\text { of the } 3^{\prime} \text { flanking region of scil interrupted by } h p h \text { in } \\
\text { pRS426 }\end{array}$ \\
\hline $\begin{array}{l}a m p^{R}, U R A 3,899 \mathrm{bp} \text { of the } 5^{\prime} \text { flanking region and } 637 \mathrm{bp} \\
\text { of the } 3^{\prime} \text { flanking region of scil interrupted by nat in } \\
\text { pRS426 }\end{array}$
\end{tabular}

Yeast two-hybrid plasmids

\begin{tabular}{|c|c|c|}
\hline pGADT7 & $\begin{array}{l}\text { LEU2, GAL4-AD, amp }{ }^{\mathrm{R}} \\
\text { PADH1::GAL4AD::TADH1 }\end{array}$ & $\begin{array}{l}\text { Takara Bio USA, Inc. } \\
\text { (Mountain View, USA) }\end{array}$ \\
\hline pA_rud3 & $\begin{array}{l}\text { LEU2, GAL4-AD, amp }{ }^{\mathrm{R}} \text {, sci1 amplified from cDNA } \\
\text { PADH1::GAL4AD::sci1::TADH1 }\end{array}$ & $\begin{array}{l}\text { Nordzieke and Kück } \\
\text { (unpublished) }\end{array}$ \\
\hline pAD-pro11 & $\begin{array}{l}\text { LEU2, GAL4-AD, amp }{ }^{\mathrm{R}} \text {, pro11 amplified from cDNA } \\
\text { PADH1::GAL4AD::pro11::TADH1 }\end{array}$ & $\begin{array}{l}\text { Bloemendal et al. } \\
\text { (2012) }\end{array}$ \\
\hline pAD-pro11(111-281) & $\begin{array}{l}\angle E U 2, \text { GAL4-AD, amp }{ }^{\mathrm{R}} \text {, pro11 (AA 111-281) amplified } \\
\text { from cDNA PADH1::GAL4AD:: pro11 111-281::TADH1 }\end{array}$ & $\begin{array}{l}\text { Bloemendal et al. } \\
\text { (2012) }\end{array}$ \\
\hline pADpro45 $\Delta T M$ & $\begin{array}{l}\angle E U 2, G A L 4-A D, \text { amp }^{R} \text {, pro } 45 \text { amplified from } c D N A \text {, } \\
\text { without TM encoding sequence } \\
\text { PADH1::GAL4AD::pro45 } \triangle T M:: T A D H 1\end{array}$ & $\begin{array}{l}\text { Teichert and Kück } \\
\text { (unpublished) }\end{array}$ \\
\hline pADRanBPM & $\begin{array}{l}\text { LEU2, GAL4-AD, amp }{ }^{\mathrm{R}} \\
\text { PADH1::GAL4AD::RanBPM::TADH1 }\end{array}$ & Tucker et al. (2009) \\
\hline
\end{tabular}




\begin{tabular}{|c|c|c|}
\hline pADFar3 & $\begin{array}{l}\text { LEU2, GAL4-AD, amp }{ }^{R}, F A R 3 \text { amplified from gDNA } \\
\text { PADH1::GAL4AD::FAR3::TADH1 }\end{array}$ & This study \\
\hline pADFar7 & $\begin{array}{l}\text { LEU2, GAL4-AD, amp }{ }^{\mathrm{R}}, F A R 7 \text { amplified from gDNA } \\
\text { PADH1::GAL4AD::FAR7::TADH1 }\end{array}$ & This study \\
\hline pADFar8 & $\begin{array}{l}\text { LEU2, GAL4-AD, amp }{ }^{\mathrm{R}}, \text { FAR8 amplified from gDNA } \\
\text { PADH1::GAL4AD::FAR8::TADH1 }\end{array}$ & This study \\
\hline pGBKT7 & $\begin{array}{l}\text { TRP1, GAL4-BD, kan } \\
\text { PADH1::GAL4BD::TADH1 }\end{array}$ & $\begin{array}{l}\text { Takara Bio USA, Inc. } \\
\text { (Mountain View, USA) }\end{array}$ \\
\hline pB_rud3 & $\begin{array}{l}\text { TRP1, GAL4-BD, kan }{ }^{\mathrm{R}} \text {, sci1 amplified from cDNA } \\
\text { PADH1::GAL4BD::sci1::TADH1 }\end{array}$ & $\begin{array}{l}\text { Nordzieke and Kück } \\
\text { (unpublished) }\end{array}$ \\
\hline pBDsci1 $1_{1-62}$ & $\begin{array}{l}\text { TRP1, GAL4-BD, } \operatorname{kan}^{\mathrm{R}} \text {, sci1 (AA 1-62) amplified from } \\
\text { cDNA PADH1::GAL4BD:: sci1 1-62::TADH1 }\end{array}$ & This study \\
\hline pBDsci1 $1-120$ & $\begin{array}{l}\text { TRP1, GAL4-BD, kan }{ }^{\mathrm{R}} \text {, sci1 (AA 1-120) amplified from } \\
\text { cDNA PADH1::GAL4BD:: sci1 1-120::TADH1 }\end{array}$ & This study \\
\hline pBDsci1 $100-155$ & $\begin{array}{l}\text { TRP1, GAL4-BD, kan }{ }^{\mathrm{R}} \text {, sci1 (AA 100-155) amplified from } \\
\text { cDNA PADH1::GAL4BD:: sci1 100-155::TADH1 }\end{array}$ & This study \\
\hline pBDsci1 $1_{63-207}$ & $\begin{array}{l}\text { TRP1, GAL4-BD, kan }{ }^{R} \text { sci1 (AA 63-207) amplified from } \\
\text { cDNA PADH1::GAL4BD:: sci163-207::TADH1 }\end{array}$ & This study \\
\hline pBDsci1 $121-207$ & $\begin{array}{l}\text { TRP1, GAL4-BD, kan }{ }^{R} \text { sci1 (AA 121-207) amplified from } \\
\text { cDNA PADH1::GAL4BD:: sci1 121-207::TADH1 }\end{array}$ & This study \\
\hline pBDsci1 $121-298$ & $\begin{array}{l}\text { TRP1, GAL4-BD, kan }{ }^{R} \text {, sci1 (AA 121-298) amplified from } \\
\text { cDNA PADH1::GAL4BD:: sci1 121-298::TADH1 }\end{array}$ & This study \\
\hline pBDsci1 $208-298$ & $\begin{array}{l}\text { TRP1, GAL4-BD, kan }{ }^{\mathrm{R}} \text { sci1 (AA 208-298) amplified from } \\
\text { cDNA PADH1::GAL4BD:: sci1 } 208-298:: T A D H 1\end{array}$ & This study \\
\hline pBDpro11 1 1-110 & $\begin{array}{l}\text { TRP1, GAL4-BD, kan }{ }^{\mathrm{R}} \text {, pro11 (AA 1-110) amplified from } \\
\text { CDNA PADH1::GAL4BD:: pro11 1-110::TADH1 }\end{array}$ & This study \\
\hline pBD-pro11(1-110) & $\begin{array}{l}\text { amp }{ }^{R}, U R A 2, \text { pro11 } c D N A \text { fragment coding for fusion } \\
\text { protein BD-PRO11(1-110) in Sall site in } p G B D U-C 3\end{array}$ & $\begin{array}{l}\text { Bloemendal et al. } \\
\text { (2012) }\end{array}$ \\
\hline pBDPRO45-FL & $\begin{array}{l}\text { TRP1, GAL4-BD, kan }{ }^{\mathrm{R}} \text {, pro45 amplified from cDNA } \\
\text { PADH1::GAL4BD::pro45::TADH1 }\end{array}$ & $\begin{array}{l}\text { Teichert and Kück } \\
\text { (unpublished) }\end{array}$ \\
\hline pBDpro45 $\Delta \mathrm{TM}$ & $\begin{array}{l}\text { TRP1, GAL4-BD, } \text { kan }^{\mathrm{R}}, \text { pro } 45 \text { amplified from cDNA, } \\
\text { without TM encoding sequence } \\
\text { PADH1::GAL4BD::pro45 } T \text { TM::TADH1 }\end{array}$ & $\begin{array}{l}\text { Teichert and Kück } \\
\text { (unpublished) }\end{array}$ \\
\hline \multicolumn{3}{|c|}{ Expression plasmids } \\
\hline p1783-1 & $\begin{array}{l}\text { amp }^{R}, \text { URA3, } \text { hyg }^{R}, \\
\text { Pgpd::egfp::TtrpC }\end{array}$ & Pöggeler et al. (2003) \\
\hline pRHN1 & $\begin{array}{l}\text { amp }^{R}, \text { URA3, } \text { nat }^{R}, \\
\text { Pgpd::Dsred::TtrpC }\end{array}$ & Janus et al. (2007) \\
\hline pHA11 & $\begin{array}{l}\operatorname{amp}^{R}, \text { URA3, } \text { nat }^{R} \\
\text { Pccg1::ha::pro11::TtrpC }\end{array}$ & $\begin{array}{l}\text { Bloemendal et al. } \\
\text { (2012) }\end{array}$ \\
\hline pHAPRO11GFP & $\begin{array}{l}\text { amp }^{R}, \text { URA3, nat } \\
\text { Pccg1::ha::pro11::egfp::TtrpC }\end{array}$ & $\begin{array}{l}\text { Herzog and Pöggeler } \\
\text { (unpublished) }\end{array}$ \\
\hline p5'scilgfp & $\begin{array}{l}a m p^{R}, U R A 3, n a t^{R}, \\
\text { 5'sci1::sci1::egfp::TtrpC }\end{array}$ & This study \\
\hline p5'sci1Dsred & $\begin{array}{l}a m p^{R}, \text { URA3, } \text { nat }^{R}, \\
\text { 5'sci1::sci1::Dsred::TtrpC }\end{array}$ & This study \\
\hline
\end{tabular}




\begin{tabular}{|c|c|c|}
\hline pscilgfp & $\begin{array}{l}\operatorname{amp}^{R}, \text { URA3, nat } \\
\text { Pccg1::sci1::egfp::TtrpC }\end{array}$ & This study \\
\hline psci1Dsred & $\begin{array}{l}\text { amp }{ }^{R}, \text { URA3, } \text { nat }^{R} \\
\text { Pccg1::sci1::Dsred::TtrpC }\end{array}$ & This study \\
\hline psci163-207_nat & $\begin{array}{l}\text { amp }{ }^{R}, \text { URA3, } \text { nat }^{R}, \\
\text { 5'sci1::sci1::sci1 } 63-207:: T t r p C\end{array}$ & This study \\
\hline psci1121-207_nat & $\begin{array}{l}a m p^{R}, \text { URA3, } \text { nat }^{R}, \\
\text { 5'sci1::sci1::sci1 121-207::TtrpC }\end{array}$ & This study \\
\hline psci1121-298_nat & $\begin{array}{l}a m p^{R}, \text { URA3, nat }{ }^{R}, \\
\text { 5'sci1::sci1::sci1 121-298::TtrpC }\end{array}$ & This study \\
\hline psci1208-298_nat & $\begin{array}{l}a m p^{R}, \text { URA3, } \text { nat }^{R} \\
\text { 5'sci1::sci1::sci1 } 208-298:: T \operatorname{trpC}\end{array}$ & This study \\
\hline $\mathrm{pRH} 2 \mathrm{~B}$ & $\begin{array}{l}a^{\prime} p^{R}, U R A 3, h y g^{R}, \\
\text { Pgpd::hh2b::tdTomato::TtrpC }\end{array}$ & Nordzieke et al. (2015) \\
\hline pRH2B_nat & $\begin{array}{l}a m p^{R}, \text { URA3, } \text { nat }^{R} \\
\text { Pgpd::hh2b::tdTomato::TtrpC }\end{array}$ & This study \\
\hline pSmPOM152GFP & $\begin{array}{l}\text { amp }{ }^{R}, \text { URA3, nat } \\
\text { Pccg1::Smpom152::egfp::TtrpC }\end{array}$ & This study \\
\hline pHEGFPRAB5 & $\begin{array}{l}\operatorname{kan}^{\mathrm{R}}, \text { URA3, hyg } \\
\text { PZttub2::egfp::Ztrab5::T Zttub2 }\end{array}$ & Kilaru et al. (2015) \\
\hline pHEGFPRAB7 & $\begin{array}{l}\text { kan }^{R}, \text { URA3, hyg } \\
\text { PZttub2::egfp::Ztrab7::T Zttub2 }\end{array}$ & Kilaru et al. (2015) \\
\hline pMito-DsRed & $\begin{array}{l}\text { amp }{ }^{R}, \text { nat }^{R}, \\
\text { Pgpd::cas2 (AA1-60)::Dsred::TtrpC }\end{array}$ & $\begin{array}{l}\text { Elleuche and Pöggeler } \\
\text { (2009) }\end{array}$ \\
\hline pAL4-Lifeact & $\begin{array}{l}a m p^{R}, \text { nat }^{R}, \\
\text { Pccg1::Lifeact::TagRFP::TtrpC }\end{array}$ & Berepiki et al. (2010) \\
\hline pDsREDKDEL & $\begin{array}{l}\text { amp }{ }^{R}, \text { URA3, nat }{ }^{R}, \text { PRO41 secretion signal (KDEL) } \\
\text { Pgpd::Dsred::pro41 secretion signal::TrpC }\end{array}$ & $\begin{array}{l}\text { Nowrousian et al. } \\
\text { (2007) }\end{array}$ \\
\hline pHA_nat & $\begin{array}{l}\text { amp }{ }^{R}, \text { URA3, } \text { nat }^{R} \\
\text { Pccg1::ha::TtrpC }\end{array}$ & This study \\
\hline pFLAG_nat & $\begin{array}{l}\text { amp }{ }^{R}, \text { URA3, } \text { nat }^{R}, \\
\text { Pccg1::flag::TtrpC }\end{array}$ & This study \\
\hline \multicolumn{3}{|l|}{ Complementation } \\
\hline pPcFGwtRN & $\begin{array}{l}a m p^{R}, \text { URA3, nat } \\
\text { Pccg1::RnFGFR1OP2::ha::TtrpC }\end{array}$ & This study \\
\hline pPcFGSNP1 & $\begin{array}{l}a m p^{R}, \text { URA3, nat } \\
\text { Pccg1::HsFGFR1OP2SNP1::ha::TtrpC }\end{array}$ & This study \\
\hline pPcFGwtHS & $\begin{array}{l}a m p^{R}, \text { URA3, nat } \\
\text { Pccg1::HsFGFR1OP2::ha::TtrpC }\end{array}$ & This study \\
\hline pFar3HA & $\begin{array}{l}\text { amp }{ }^{R}, \text { URA3, } \text { nat }^{R}, \\
\text { Pccg1::FAR3::ha::TtrpC }\end{array}$ & This study \\
\hline pFar7FLAG & $\begin{array}{l}a m p^{R}, \text { URA3, } \text { nat }^{R}, \\
\text { Pccg1::FAR7::flag::TtrpC }\end{array}$ & This study \\
\hline Others & & \\
\hline $\begin{array}{l}\text { pRRLsinhCMV-IG- } \\
\text { FGFR1OP2_RN }\end{array}$ & $\begin{array}{l}\text { Deletion in the } 3^{\prime} S I N-L T R \text { promoter, WPRE } \\
\text { posttranscriptional regulatory element, internal } \\
\text { ribosome entry site fused to GFP IRES-GFP, wt Rattus }\end{array}$ & Lin et al. (2010) \\
\hline
\end{tabular}


norvegicus FGFR1OP2, hCMV promoter, central

polypurine tract/central termination sequence

CPPT/CTS, chimeric 5' LTR 5'ChimLTR

\begin{tabular}{|c|c|c|}
\hline $\begin{array}{l}\text { pRRLsinhCMV-IG- } \\
\text { FGFR1OP2_HS }\end{array}$ & $\begin{array}{l}\text { Deletion in the 3'SIN-LTR promoter, WPRE } \\
\text { posttranscriptional regulatory element, internal } \\
\text { ribosome entry site fused to GFP IRES-GFP, wt Homo } \\
\text { sapiens FGFR1OP2/wit3.OB, hCMV promoter, central } \\
\text { polypurine tract/central termination sequence } \\
\text { CPPT/CTS, chimeric 5' LTR 5'ChimLTR }\end{array}$ & Lin et al. (2010) \\
\hline $\begin{array}{l}\text { pRRLsinhCMV-IG- } \\
\text { FGFR1OP2-SNP1_HS }\end{array}$ & $\begin{array}{l}\text { Deletion in the } 3^{\prime} S I N-L T R \text { promoter, WPRE } \\
\text { posttranscriptional regulatory element, internal } \\
\text { ribosome entry site fused to GFP IRES-GFP, wt Homo } \\
\text { sapiens FGFR1OP2/wit3.OB-SNP1, hCMV promoter, } \\
\text { central polypurine tract/central termination sequence } \\
\text { CPPT/CTS, chimeric } 5^{\prime} \text { LTR } 5^{\prime} C h i m L T R\end{array}$ & Lin et al. (2010) \\
\hline
\end{tabular}

The native promoter and terminator of sci1 are named 5'sci1 and 3'sci1, respectively. Other promoters and terminators are marked with a $P$ or $T$, respectively; Pgpd: promoter of the glycerin aldehyde 3-phosphate dehydrogenase (Aspergillus nidulans), TtrpC: Promoter of the trpC gene of the anthranilate synthase pathway (A. nidulans); Pccg1: promoter of the clock controlled gene 1 of $N$. crassa; amp ${ }^{R}$, ampicillin resistance; $\mathrm{kan}^{R}$, kanamycin resistance; $\mathrm{hyg}^{R}$, resistance to hygromycin, hygromycin B phosphotransferase; $n a t^{R}$ : resistance to nourseothricin, nourseothricin $\mathrm{N}$-acetyl transferase, The amino-acid (AA) region encoded by the fragment shown is given in brackets or is written in lower case.

\subsubsection{Primers}

Table 4: List of primers used in this study

\begin{tabular}{|c|c|c|}
\hline Oligomer name & Sequence $\left(5^{\prime} \rightarrow 3^{\prime}\right)$ & Binding Position \\
\hline Deletion & & \\
\hline rud3_5'f & $\begin{array}{l}\text { GTAACGCCAGGGTTTTCCCAGTCACGACG } \\
\text { GCTAGCTATCAACATCATCG }\end{array}$ & $\frac{\text { pRS426 }}{\text { sci1-880 to }-900}$ \\
\hline rud3_5'r & $\frac{\text { CCAAAAATGCTCCTTCAATATCAGTTAAC }}{\text { GTCCTCTTCGTCTTCGTTGT }}$ & $\frac{h p h}{s c i 1}-1$ to -21 \\
\hline rud3_3'f & $\frac{\text { CCAGCACTCGTCCGAGGGCAAAGGAATAG }}{\text { ATGAACGTACGTACGTACGG }}$ & 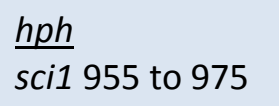 \\
\hline Sci1_3'f_nat & $\frac{\text { CGCTCTACATGAGCATGCCCTGCCCCTGA }}{\text { ATGAACGTACGTACGTACGG }}$ & $\frac{\text { nat }}{\text { sci1 } 955 \text { to } 975}$ \\
\hline rud3_3'r & $\frac{\text { GCGGATAACAATTTCACACAGGAAACAGC }}{\text { TTACGGAGTTGGTATCAAGG }}$ & $\frac{\text { pRS426 }}{\text { sci1 } 1613 \text { to } 1593}$ \\
\hline hph-f & GTTAACTGATATTGAAGGAGCATTTTTTGG & PtrpC 1 to 27 \\
\hline
\end{tabular}


Materials and Methods

$\begin{array}{lll}\text { nat-1r } & \text { TCAGGGGCAGGGCATGCTCA } & \text { nat } 570 \text { to } 551 \\ \text { hph-r } & \text { GTTAACTGGTTCCCGGTCGGCATCTACTC } & \text { hph } 1418 \text { to } 1390\end{array}$

\section{Expression}

\begin{tabular}{|c|c|c|}
\hline pRSccg1 & $\frac{\text { GTAACGCCAGGGTTTTCCCAGTCACGACG }}{\text { TAGAAGGAGCAGTCCATCTG }}$ & $\frac{\text { pRSnat/pRShyg }}{\text { Pccg1 } 1 \text { to } 922}$ \\
\hline Pccg1-r & TTTGGTTGATGTGAGGGGTT & Pccg1 902 to 922 \\
\hline sci1ccg1_f & $\frac{\text { CACTTTCACAACCCCTCACATCAACCAAA }}{\text { ATGAACGGCATGCATGCTGG }}$ & $\frac{\text { Pccg1 }}{\operatorname{sci} 11 \text { to } 20}$ \\
\hline Pccg1HAr_TrpC & $\frac{\text { TTGATGATTTCAGTAACGTTAAGTGGATC }}{\text { ACTAGTTCTAGTTGAACCAGAACCAGC }}$ & $\frac{\text { TtrpC }}{\text { ha } 25 \text { to } 52}$ \\
\hline Pccg1FLAGr_TtrpC & $\frac{\text { TTGATGATTTCAGTAACGTTAAGTGGATC }}{\text { TGAACCCTTGTCATCGTCATCCTTGT }}$ & $\frac{\text { TtrpC }}{\text { Flag } 84 \text { to } 59}$ \\
\hline TrpC_F & GATCCACTTAACGTTACTGAAATCATCAAA & $\operatorname{TtrpC} 1$ to 20 \\
\hline pRS426GFPrev & $\frac{\text { GCGGATAACAATTTCACACAGGAAACAGC }}{\text { TCGAGTGGAGATGTGGAGTG }}$ & $\frac{\mathrm{pRSnat} / \mathrm{pRShyg}}{\operatorname{TtrpC} 747 \text { to } 767}$ \\
\hline pRS426GPDf2 & $\frac{\text { GTAACGCCAGGGTTTTCCCAGTCACGACG }}{\text { GTACAGTGACCGGTGACTCT }}$ & $\frac{\text { pRSnat/pRShyg }}{\text { Pgpd } 1 \text { to } 20}$ \\
\hline 5'sci1_rev & GTCCTCTTCGTCTTCGTTGT & sci1 -20 to -1 \\
\hline sci1_t1f & $\frac{\text { AAGAAACAGACAACGAAGACGAAGAGGAC }}{\text { ATGTCACAGTCTCTGACAAA }}$ & $\frac{5^{\prime} \text { flank of sci1 }}{\text { sci1 } 185 \text { to } 205}$ \\
\hline sci1_t3f & $\frac{\text { AAGAAACAGACAACGAAGACGAAGAGGAC }}{\text { ATGCTCAAGAAATTCCACGA }}$ & $\frac{5^{\prime} \text { flank of sci1 }}{\text { sci1 } 438 \text { to } 458}$ \\
\hline sci1_t5f & $\frac{\text { AAGAAACAGACAACGAAGACGAAGAGGAC }}{\text { ATGGCGATGCCGGAACTGCC }}$ & $\frac{5^{\prime} \text { flank of sci1 }}{\text { sci1 } 699 \text { to } 719}$ \\
\hline sci1_t7r & $\frac{\text { GTGAACAGCTCCTCGCCCTTGCTCACCAT }}{\text { GCGTTTCCAGAACCTCAGCT }}$ & $\frac{\text { egfp }}{\text { sci1 } 720 \text { to } 700}$ \\
\hline FLAGf & TCTAGAACTAGTATGGACTACAAAG & flag 1 to 25 \\
\hline HAf & TCTAGATACCCATACGATGTTCCAG & ha 1 to 25 \\
\hline
\end{tabular}




\begin{tabular}{|c|c|c|}
\hline GFP-f & ATGGTGAGCAAGGGCGAGGAGC & egfp 1 to 22 \\
\hline DsRed-f & $\begin{array}{l}\text { ATGGCCTCCTCCGAGGACGTCATCAAGGAGTTC } \\
\text { ATGCG }\end{array}$ & Dsred 1-38 \\
\hline scilgfp_r & $\frac{\text { GTGAACAGCTCCTCGCCCTTGCTCACCAT }}{\text { GGAAACCGGCGGCTGTGGTA }}$ & $\begin{array}{l}\text { egfp } \\
\text { sci1 } 953 \text { to } 973\end{array}$ \\
\hline sci1dsRed_r & $\frac{\text { TCCTTGATGACGTCCTCGGAGGAGGCCAT }}{\text { GGAAACCGGCGGCTGTGGTA }}$ & 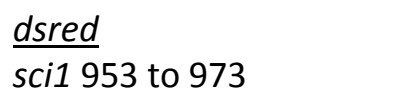 \\
\hline Pom_ccgf & $\frac{\text { CACTTTCACAACCCCTCACATCAACCAAA }}{\text { ATGGCCGGACCAAGACGACC }}$ & $\frac{\text { Pccg1 }}{\text { Smpom152 } 1 \text { to } 20}$ \\
\hline Pom_gfpr & $\frac{\text { GTGAACAGCTCCTCGCCCTTGCTCACCAT }}{\text { TTGGTACTTCAACAAACGAC }}$ & $\begin{array}{l}\frac{\text { eqfp }}{\text { Smpom152 } 3898 \text { to } 3917}\end{array}$ \\
\hline Nat-f & ATGGCCACCCTCGACGACACGGCTTACCG & nat 1 to 29 \\
\hline
\end{tabular}

\section{Verification}

\begin{tabular}{|c|c|c|}
\hline sci1_vf & AGGTCAGCTCCCAACAGCAAGT & scil -1103 to -1083 \\
\hline sci1_vr & TTGGGATCGGTTCGTGGAGA & sci1 1846 to 1826 \\
\hline hph1 & CCCGCTGTTCTGGAGCCGGTC & hph 325 to 345 \\
\hline hph2 & GACCGGCTCCAGAACAGCGGG & hph 345 to 325 \\
\hline sosci1_f & CTGGTAGGCACCTAGCCCAC & sci1 -1219 to -1199 \\
\hline sosci1_r & GCCGTGGATGAATGGAGATC & sci1 -589 to -569 \\
\hline pro11-21 & AAGCGCGCTTGCCAGTCGCTGC & pro11 -783 to -762 \\
\hline Pro115r_wohph & AGTTGTGCGGTTGTCGTTGGTCGAAGC & pro11 -1 to -27 \\
\hline TrpC1 & TCACCGCCTGGACGACTAAACC & TtrpC 95 to 74 \\
\hline Pro110RF-f & ATGGGCACCAACGGCGTTCATGT & pro11 1 to 23 \\
\hline Pro110RF-r & CCTCGCATACACCTTGACCATGC & pro11 2714 to 2692 \\
\hline
\end{tabular}




\begin{tabular}{|c|c|c|}
\hline pro11-kor & ACGATCAGCCTCGGAAAGACCGC & pro11 3586 bis 3564 \\
\hline hph3 & ACTCGTCCGAGGGCAAAGGAATAG & hph 1003 to 1026 \\
\hline \multicolumn{3}{|l|}{ Sequencing } \\
\hline sci_f & TCGCAAGTCATGTACACCAT & scil 420 to 440 \\
\hline sci_r & TCGCGCAGCCGACTATTCTC & sci1 554 to 534 \\
\hline \multicolumn{3}{|c|}{ Yeast two-hybrid } \\
\hline Pro11-110f & $\frac{\text { AGGAGGACCTGCATATGGCCATGGAGGCC }}{\text { ATGGGCACCAACGGCGTTCA }}$ & $\frac{\mathrm{pGBKT7}}{\text { pro11 } 1 \text { to } 20}$ \\
\hline Pro11-110r & $\frac{\text { CGCTGCAGGTCGACGGATCCCCGGGAATT }}{\text { CAACTCCCTTCTCTTTACCA }}$ & $\frac{\text { pGBKT7 }}{\text { pro11 } 401 \text { to } 381}$ \\
\hline sci1_Y1f & $\frac{\text { CCGAATTCCCGGGGATCCGTCGACCTGCA }}{\text { ATGAACGGCATGCATGCTGG }}$ & $\frac{\mathrm{pGBKT7}}{\text { sci1 } 1 \text { to } 20}$ \\
\hline sci1_Y1r & $\frac{\text { CCAAGGGGTTATGCTAGTTATGCGGCCGC }}{\text { TCACCTTGCTCGCCCGCGCAC }}$ & $\frac{\text { pGBKT7 }}{\text { sci1 } 186 \text { to } 169}$ \\
\hline sci1_Y2r & $\frac{\text { CCAAGGGGTTATGCTAGTTATGCGGCCGC }}{\text { TCAGGTGTACATGACTTGCGAGT }}$ & $\frac{\text { pGBKT7 }}{\text { sci1 } 438 \text { to } 418}$ \\
\hline sci1_Y2f & $\frac{\text { CCGAATTCCCGGGGATCCGTCGACCTGCA }}{\text { TCACAGTCTCTGACAAATGA }}$ & $\frac{\text { pGBKT7 }}{\text { sci1 } 185 \text { to } 205}$ \\
\hline sci1_Y3r & $\frac{\text { CCAAGGGGTTATGCTAGTTATGCGGCCGC }}{\text { TCAGCGTTTCCAGAACCTCAG }}$ & $\frac{\text { pGBKT7 }}{\text { sci1 } 699 \text { to } 719}$ \\
\hline sci1_Y3f & $\frac{\text { CCGAATTCCCGGGGATCCGTCGACCTGCA }}{\text { ATGCTCAAGAAATTCCACGA }}$ & $\frac{\text { pGBKT7 }}{\text { sci1 } 438 \text { to } 458}$ \\
\hline sci1_Y4r & $\frac{\text { CCAAGGGGTTATGCTAGTTATGCGGCCGC }}{\text { TCAGGAAACCGGCGGCTGTG }}$ & $\frac{\text { pGBKT7 }}{\text { sci1 } 975 \text { to } 955}$ \\
\hline sci1_Y4f & $\frac{\text { CCGAATTCCCGGGGATCCGTCGACCTGCA }}{\text { ATGGCGATGCCGGAACTGCC }}$ & $\frac{\mathrm{pGBKT7}}{\text { sci1 } 699 \text { to } 719}$ \\
\hline sci1_Y5f & $\frac{\text { CCGAATTCCCGGGGATCCGTCGACCTGCA }}{\text { AAATTCTCTCGCGACGCCAA }}$ & $\frac{\mathrm{pGBKT7}}{\text { sci1 } 301 \text { to } 320}$ \\
\hline sci1_Y5r & $\frac{\text { CCAAGGGGTTATGCTAGTTATGCGGCCGC }}{\text { ACTATTCTCCGCGCGCGCCT }}$ & $\frac{\mathrm{pGBKT7}}{\text { sci1 } 446 \text { to } 465}$ \\
\hline
\end{tabular}




\begin{tabular}{|c|c|c|}
\hline Far7ph_f & AATGAGTGACCAAATCAACGT & FAR7 1 to 20 \\
\hline Far8phf & AATGGCTATTAACCAGGCACA & FAR8 1 to 20 \\
\hline Far8phr & TTATATTTTAAATCTTGTGCAAAA & FAR8 1551 to 1571 \\
\hline \multicolumn{3}{|c|}{ quantitative reverse-transcription PCR } \\
\hline RTsci1f & GAGCTGAGGTTCTGGAAACG & sci1 680 to 699 \\
\hline RTsci1r & ССАСТТССАСТСССАСТСАТ & sci1 915 to 896 \\
\hline SSU-f & ATCCAAGGAAGGCAGCAGGC & Nc gDNA SC8 93867 to 93886 \\
\hline SSU-r & TGGAGCTGGAATTACCGCG & Nc gDNA SC8 94028 to 94046 \\
\hline
\end{tabular}

\section{Complementation studies}

\begin{tabular}{|c|c|c|}
\hline Far3_f & $\frac{\text { CACTTTCACAACCCCTCACATCAACCAAA }}{\text { ATGAATAGCGGTGGTAGTGA }}$ & $\frac{\text { Pccg1 }}{\text { FAR3 } 1 \text { to } 20}$ \\
\hline Far3_r & $\frac{\text { CCAGCGTAATCTGGAACATCGTATGGGTA }}{\text { TTTCAGTACCTGGTACGTGT }}$ & $\frac{h a}{F A R 3} 612$ to 593 \\
\hline Far7_f & $\frac{\text { CACTTTCACAACCCCTCACATCAACCAAA }}{\text { ATGAGTGACCAAATCAACGT }}$ & $\frac{\text { Pccq1 }}{\text { FAR7 } 1 \text { to } 20}$ \\
\hline Far7flag_r & $\frac{\text { TGGTCTTTGTAGTCCATACTAGTTCTAGA }}{\text { ATCTTGTGCATGTAACCTCA }}$ & $\frac{\text { flaq }}{\text { FAR7 } 663 \text { to } 644}$ \\
\hline FG_RNf & ATGAGCTGCACCATTGAGAA & Fgfr1op2/wit3.0ß cDNA 1 to 20 \\
\hline FG_RNr & GCTCTTCCTCAGACTCAGGT & $\begin{array}{l}\text { Fgfr1op2/wit3.0ß cDNA } 759 \text { to } \\
740\end{array}$ \\
\hline ccg1_FGRN & $\frac{\text { GCAAGTGCCTTCTCAATGGTGCAGCTCAT }}{\text { TTTGGTTGATGTGAGGGGTT }}$ & $\frac{\text { Fgfr1op2 }}{\text { Pccg1 } 922 \text { to } 903}$ \\
\hline Haf_FGNR & $\frac{\text { CTAACAGTGACCTGAGTCTGAGGAAGAGC }}{\text { TACCCATACGATGTTCCAGA }}$ & $\frac{\text { Fgfr1op2 }}{\text { ha } 1 \text { to } 20}$ \\
\hline
\end{tabular}

Overhangs for homologous recombination in yeast are underlined. The region of overhangs homologous to the respective plasmid or gene is given and also underlined. Additional bp for generation of a stop codon (if required) are shaded in gray. 


\subsubsection{Chemicals and materials}

\subsubsection{Chemicals}

Acetic acid (Roth $\mathrm{GmbH}, 3738.2$ ), acetonitrile (Roth $\mathrm{GmbH}, 8825$ ), acrylamide (Rotiphorese ${ }^{\circledR}$ Gel 40 37,5:1) (Roth GmbH, 3029.1), adenine (Sigma-Aldrich, 01830-50G), agar-agar (Roth GmbH, 5210.2), agar-agar SERVA high gel-strength (SERVA, 11396.03), agarose (Biozym Scientific GmbH, 840004), albumin bovine (Sigma-Aldrich, A9647-50G), ammonium bicarbonate (Sigma-Aldrich, A6141), ammonium chloride (VWR International, BDH0208-500G), ammonium sulfate (AppliChem, A1032.1000), ammonium persulfate (APS) (Roth $\mathrm{GmbH}, 9592.3$ ), ampicillin (Sigma-Aldrich, A951825G), bacto-yeast-extract (Oxoid LTD., LP0021), ß-mercaptoethanol (Roth GmbH, 4227.1), bio malt maize extract (Brau-Partner Kling, 115), biotin (Sigma-Aldrich, B4501-1G), boric acid (Roth GmbH, 6943.1), bromophenol blue (AppliChem, A3640.0005), calcium chloride (Roth GmBH, CN92.1), calcium chloride (Roth $\mathrm{GmbH}, \mathrm{CN} 92.1$ ), calcium chloride dihydrate (Roth $\mathrm{GmbH}, 5239.1$ ), CFW (calcoflour white stain) (Sigma-Aldrich, 18909-100ML-F), citric acid monohydrate (Roth $\mathrm{GmbH}$, 3958.1), CMAC (7-amino-4-chloromethyl-coumarin) (Invitrogen, C-2110), copper (II) sulfate 5hydrate (Roth GmbH, P024.1), Coomassie ${ }^{\circledR}$ brilliant blue R-250 (Serva, 17525), CSM-Ade-His-LeuTrp-Ura (MP Biomedicals, 4550-122), DAPI (4'-6-diamidino-2-phenylindole) (AppliChem, A1001.0010), deoxynucleotide triphosphates (dNTPs) (Thermo Scientific, R0191), deoxyribonucleic acid sodium salt from salmon testis (ss-DNA) (Sigma Aldrich, D1626-1G), disodium hydrogen phosphate (Merck-Millipore, 1065855000), DMF (dimethylformamide) (Roth GmbH, T921.1) DMSO (dimethyl sulfoxide) (Merck Millipore, 1029310500), DTT (1,4-dithiothreitol) (AppliChem, A1101.0025), EDTA (ethylenediamine tetraaceticacid disodium salt dihydrate) (Roth $\mathrm{GmbH}$, 8043.2), EDTA-free protease inhibitor cocktail (Roche, 04693132001), ethanol (VWR International, 20821.321), ethidium bromide (EtBr) (Sigma-Aldrich, 46065), FM 4-64 (Invitrogen, F34653), formaldehyde (Roth $\mathrm{GmbH}, 4979.2$ ), formic acid (Merck Millipore, 1002641000), GeneRuler ${ }^{\mathrm{TM}}$ DNA Ladder Mix (Thermo Scientific, SM0331), GeneRuler ${ }^{\mathrm{TM}}$ 1kb DNA Ladder (Thermo Scientific, SM0313), glucose (Applichem, A3617.1000), glycerol (VWR International, 24388.295), glycine (Roth GmbH, 0079.1), glycerin ROTIPURAN ${ }^{\circledR}$ (Roth GmbH, 200-289-5), HEPES (4-(2-hydroxyethyl)1-piperazineethanesulfonicacid) (Roth $\mathrm{GmbH}, 9105.4$ ), hydrochloric acid (Roth $\mathrm{GmbH}, 4625.2$ ), hydrogen peroxide 30\% $\left(\mathrm{H}_{2} \mathrm{O}_{2}\right)$ (Merck Millipore, 8.22287.2500), hygromycin B (Merck-Millipore, 400051-10MU), iodoacetamide (Sigma-Aldrich, I1149), iron (II) chloride (Roth GmbH, 231-753-5), iron(II) sulfate heptahydrate (Sigma-Aldrich, 31236), imidazole (Roth $\mathrm{GmbH}, \mathrm{X998.1}$ ), isopropanol (AppliChem, A0900,2500GL), kanamycin sulfate (Sigma-Aldrich, 60615), L-arginine (Roth, 3144.1), L-histidine (Applichem, A3738.0100), L-leucine (AppliChem, A1426.0100), lithium acetate (Roth $\mathrm{GmbH}, 5447.1$ ), magnesium chloride hexahydrate (Merck Millipore, 1.05833.1000), L-tryptophan (MP Biomedicals, 4061-012), magnesium sulfate heptahydrate (Roth $\mathrm{GmbH}, \mathrm{P027.2}$ ), maize flour (Mühle Levers, Bochum, Germany and Mühle Udo Kellner, Schimberg, Germany), manganese (II) chloride tetrahydrate (Roth $\mathrm{GmbH}, \mathrm{T} 881.1$ ), manganese (II) sulfate monohydrate (Roth $\mathrm{GmbH}$, 4487.1), Maxima SYBR Green/ROX qPCR master mix (2x) (Thermo Fisher, K0221), methanol (VWR

International, 20864.320), Nonidet $^{\circledR}$ (NP40) (AppliChem A2239.0025), nourseothricin 
(WernerBioAgents, 5004000), NuPAGE ${ }^{\text {TM }}$ LDS Sample Buffer (4x) (Thermo Scientific Invitrogen, NP0007), PageRuler ${ }^{T M}$ prestained protein ladder (Thermo Scientific, 26616), PEG 4000 (Polyethylene Glycol 4000) (AppliChem, A1249.1000), PEG 6000 (Polyethylene Glycol 6000) (SigmaAldrich, 81255), phenol (Applichem, A1153.0500), phosphoric acid (Roth GmbH, 6366.1), 5x phusion HF buffer (Thermo Scientific, F518), PMFS (phenylmethylsulfonyl fluoride) (Serva, 32395), potassium acetate (Merck Millipore, 1.04820.1000), potassium chloride (AppliChem, A3582,1000), potassium dihydrogen phosphate (Merck Millipore, 1.04873.1000), potassium hydroxide (Roth $\mathrm{GmbH}$, 6751.1), potassium nitrate (Merck Millipore, 1.05063.1000), potassium nitrite (SigmaAldrich, 31443), RNA gel loading dye (2x) (Thermo Scientific, R0641), Rotiphorese Gel 30 (Roth $\mathrm{GmbH}, 3029.1$ ), saccharose (Roth $\mathrm{GmbH}, 200-334-9$ ), Roti ${ }^{\circledR}$-Quant (5x) (Roth, K015.1), SDS (sodium dodecyl sulfate) (Roth $\mathrm{GmbH}, 4360.2$ ), Difco $^{\text {TM }}$ skim milk (BD Biosciences, 232100), sodium acetate (Roth $\mathrm{GmbH}, 6773.2$ ), sodium chloride (AppliChem, A3597,1000), sodium dihydrogen phosphate monohydrate (Merck Millipore, 1.06346.1000), sodium hydroxide (VWR International, 28244.295), Difco $^{\text {TM }}$ soluble starch (BD Biosciences, 217820), sorbitol (Roth $\mathrm{GmbH}, 6213.1$ ), sucrose (AppliChem, A4734.1000), TEMED (N,N,N',N'- tetramethylethylenediamine) (Roth GmbH, 2367.1), TRIS PUFFERAN $^{\circledR}$ (Tris-hydroxymethyl-aminomethane) (Roth $\mathrm{GmbH}, \mathrm{AE15}$.2), TRIS hydrochlorid (Roth $\mathrm{GmbH}$, 9090.3), Trizol (Invitrogen,15596026), trypsin resuspension dilution buffer (Promega, V542A), tryptone/peptone (Roth $\mathrm{GmbH}, 8952.2)$, Tween 20 ${ }^{\circledR}$ (AppliChem, A4974.0100), uracil (Sigma-Aldrich, U1128), urea (Roth $\mathrm{GmbH}, 2317.3$ ), Water for chromatography (Merck Millipore, 7732-18-5), xylene cyanol (AppliChem, A4976.0005), yeast extract (Thermo Scientific, LP0021), Difco $^{\mathrm{TM}}$ yeast nitrogen base w/o amino acids and ammonium sulfate (BD Biosciences, 233520), zinc chloride (Sigma-Aldrich, 14424), zinc sulfate heptahydrate (Roth GmbH, K301.1)

\subsubsection{Materials}

Amersham Hybond ${ }^{\mathrm{TM}} \mathrm{N}^{+}$(GE Healthcare, RPN203B), Amersham Hyperfilm ${ }^{\mathrm{TM}}$ ECL (GE Healthcare, 28906837 ), Amersham ${ }^{\mathrm{TM}}$ Protran $^{\mathrm{TM}}$ Nitrocellulose Blotting Membrane 0.45 $\mu \mathrm{m}$ (GE Healthcare, 10600003), anti-HA-agarose beads (Merck, A2095), BioDocAnalyzer (Biometra, Germany), disposable cuvettes 10x4x45 (Sarstedt, 67.742), Electroporation cuvettes (VWR International, 7321137), Empore ${ }^{\mathrm{TM}}$ Solid Phase Extraction Disk C18/47mm (Neuss, 2215, 3M), Flat Optical 8-Cap Strip $0.2 \mathrm{ml}$ (Biozym, 712100), Frit (ROBU glass, pore size1), GFP-Trap_A (Chromotek, gta-20), glass beads $\varnothing 0.25-0.5 \mathrm{~mm}$ (Roth $\mathrm{GmbH}, \mathrm{A} 553.1$ ), glass beads $\varnothing$ 2.85-3.45 mm (Roth $\mathrm{GmbH}, \mathrm{A} 557.1), 0.2$ $\mathrm{ml}$ Low Profile 8er-Strips (Biozym, 621935), Protein LoBind Tubes (Eppendorf, 0030108116), Sepharose A (GE Healthcare, 17078001), sterile filter 0.45/0.2 $\mu \mathrm{m}$ (Sarstedt, 83.1826/83.1826.001), Whatman Paper B002 580x600 mm (Schleicher \& Schuell, 88-3852)

\subsubsection{Kits}

AlkPhos Direct Labelling and Detection Kit (Amerham, GE Healthcare, RPN3680), CloneJET PCR Cloning Kit (Thermo Scientific, K1231), FastGene ${ }^{\circledR}$ Plasmid Mini Kit (Nippon Genetics Co., Ltd, FG90402), Immobilon ${ }^{\mathrm{TM}}$ Western HRP Substrate (Merck Millipore, WBKL S0100), QIAprep Spin Miniprep Kit (Qiagen, 27106), QIAquick Gel Extraction Kit (Qiagen, 28704), QIAquick PCR 
Purification Kit (Qiagen, 28104), QuantiTect Rev. Transcription Kit (Qiagen, 205311), Rapid DNA Dephos \& Ligation Kit (Roche, 04898117001), Transcriptor High Fidelity cDNA Synthesis Kit (Roche, 05091284001), ZR Fungal/Bacterial DNA MiniPrep ${ }^{\text {TM }}$ (Zymo Research, D6005)

\subsubsection{Enzymes}

DNase I (Thermo Scientific, EN0521), HotstarTaq Master Mix (Qiagen, 203443), In-fusion HD enzyme premix 5x (Clontech 639648), Lysozyme (Serva, 28262.03), Natuzym (Schliessmann, 5090), Pfu polymerase (Promega $\mathrm{GmbH}, \mathrm{M7741}$ ), Phusion High-Fidelity DNA polymerase (Thermo Scientific, F-530L), restriction endonucleases (Thermo Scientific), RNase A (AppliChem, A2760,0100), T4 DNA ligase (Thermo Scientific, EL0011), TopTaq DNA Polymerase (Qiagen, 200203), Trypsin NB sequencing grade modified from porcine panchreas (Serva, 37283.01)

\subsubsection{Antibodies}

The primary antibodies listed in table 5 were used for detection of fusion proteins. If required a secondary antibody was applied. For detection, the secondary antibody or the anti-GFPHRP antibody were used, which are coupled to horseradish peroxidase catalyzing oxidation of luminol and emitting light.

Table 5: Antibodies used for detection of eGFP, DsRED, HA or FLAG-tagged fusion proteins

\begin{tabular}{lllll} 
Antibody & Supplier, Order Number & Host & Dilution range & Clonality \\
\hline $\begin{array}{l}\text { Primery antibody } \\
\text { anti-FLAG M2 }\end{array}$ & Sigma-Aldrich, F3165, & Mouse & $1: 12000$ & Monoclonal \\
\hline anti-HA, clone-HA7 & Sigma-Aldrich, H9658 & Mouse & $1: 3000$ & Monoclonal \\
anti-RFP & GenScript, A00682 & Rabbit & $1: 2000$ & Polyclonal \\
\hline $\begin{array}{l}\text { anti-GFPHRP } \\
\text { Secondary antibody }\end{array}$ & Acris Antibodies, R1091HRP & Goat & $1: 3000$ & Polyclonal \\
\hline anti-Mouse & Dianova/Jackson & & & \\
& ImmunoResearch, 115-035-003 & & $1: 10000$ & Polyclonal \\
anti-Rabbit & Life Technologies, G21234 & Goat & $1: 2000$ & Polyclonal
\end{tabular}

\subsubsection{Buffers and solutions}

\section{Medium supplements}

E.coli

Ampicillin stock solution: $\quad 100 \mathrm{mg} / \mathrm{mL}$ in $\mathrm{H}_{2} \mathrm{O}$

Kanamycin stock solution: $\quad 30 \mathrm{mg} / \mathrm{mL}$ in $\mathrm{H}_{2} \mathrm{O}$ 


\section{S. cerevisiae}

Adenine stock solution:

Histidine stock solution:

Leucine stock solution:

Tryptophan stock solution:

Uracil stock solution:

\section{S. macrospora}

Hygromycin B stock solution: Nourseothricin stock solution: SWG trace elements solution:

Arginine stock solution:

Biotin stock solution:

CMS trace elements solution:
$0.02 \%(\mathrm{w} / \mathrm{v})$ adenine in $\mathrm{H}_{2} \mathrm{O}_{\text {dest }}$

$1 \%(\mathrm{w} / \mathrm{v})$ histidine in $\mathrm{H}_{2} \mathrm{O}_{\text {dest }}$

$1 \%(\mathrm{w} / \mathrm{v})$ leucine in $\mathrm{H}_{2} \mathrm{O}_{\text {dest }}$

$0.02 \%(w / v)$ tryptophan in $\mathrm{H}_{2} \mathrm{O}_{\text {dest }}$

$0.02 \%(w / v)$ uracil in $\mathrm{H}_{2} \mathrm{O}_{\text {dest }}$ $\sim 440 \mathrm{MU} / \mathrm{mL}$

$200 \mathrm{mg} / \mathrm{mL}$

$5 \%(w / v)$ citric acid, 5\% (w/v) $\mathrm{ZnSO}_{4} \times 7 \mathrm{H}_{2} \mathrm{O}, 1 \%(\mathrm{w} / \mathrm{v})$, $\mathrm{Fe}\left(\mathrm{NH}_{4}\right)_{2}\left(\mathrm{SO}_{4}\right)_{2} \times 6 \mathrm{H}_{2} \mathrm{O}, 0.25 \%(\mathrm{w} / \mathrm{v}) \mathrm{CuSO}_{4} \times 5 \mathrm{H}_{2} \mathrm{O}, 0.05 \%(\mathrm{w} / \mathrm{v})$ $\mathrm{MnSO}_{4} \times \mathrm{H}_{2} \mathrm{O}, 0.05 \%$ (w/v) $\mathrm{H}_{3} \mathrm{BO}_{3}, 0.05 \%$ (w/v) $\mathrm{Na}_{2} \mathrm{MoO}_{4} \times 2 \mathrm{H}_{2} \mathrm{O}$, $1 \%(\mathrm{v} / \mathrm{v})$ chloroform

$100 \mathrm{mg} / \mathrm{mL}$ in $\mathrm{H}_{2} \mathrm{O}_{\text {dest }}$ $0.01 \%(\mathrm{w} / \mathrm{v})$ biotin, $50 \%(\mathrm{v} / \mathrm{v})$ ethanol

Westergaard solution (1x): $0.1 \%(w / v) \mathrm{KNO}_{3}, 0.1 \%(w / v) \mathrm{KH}_{2} \mathrm{PO} 4$, $0.05 \%(\mathrm{w} / \mathrm{v}) \mathrm{MgSO}_{4}$ heptahydrate, $0.01 \%(\mathrm{w} / \mathrm{v}) \mathrm{NaCl}, 0.01 \%(\mathrm{w} / \mathrm{v})$ $\mathrm{CaCl}_{2}, 0.01 \%(\mathrm{v} / \mathrm{v}) \quad 0.01 \%$ SWG trace-elements solution $10 \mathrm{mg} / \mathrm{L} \mathrm{ZnSO}_{4}, 10 \mathrm{mg} / \mathrm{L} \mathrm{Fe}(\mathrm{II}) \mathrm{Cl}_{2}, 10 \mathrm{mg} / \mathrm{L} \mathrm{MnCl}_{2}$

\section{PCR and gel electrophoresis:}

DNA

dNTP-Mix (10 mM):

of each nucleotide dATP, dCTP, dGTP, dTTP, $10 \mathrm{mM}$ in $\mathrm{H}_{2} \mathrm{O}$

DNA loading dye $(6 x)$ :

$\operatorname{TBE}(10 \mathrm{x})$ :

Agarose gel:

EtBr stock solution:

\section{RNA}

MOPS buffer $(10 \mathrm{x})$ :

0.2 M MOPS (pH 7.0), $50 \mathrm{mM}$ sodium acetate, $10 \mathrm{mM}$ EDTA (pH 8.0)

Agarose gel with MOPS:

\section{Purification of nucleic acids}

BD solution I:

BD solution II:

$1.25 \%(\mathrm{w} / \mathrm{v})$ agarose in $1 \times$ MOPS/0.8\% (v/v) formaldehyde (37\%)
$1 \mathrm{M}$ Tris adjusted with $\mathrm{HCl}, 1 \mathrm{M}$ boric acid, $20 \mathrm{mM}$ EDTA, $\mathrm{pH} 8.3$ $1 \%$ or $2 \%(\mathrm{w} / \mathrm{v})$ agarose in $1 \times \mathrm{TBE}$ $10 \mathrm{mg} / \mathrm{mL} \mathrm{EtBr}$ in $\mathrm{H}_{2} \mathrm{O}$

BD solution III:

$50 \mathrm{mM}$ glucose, $10 \mathrm{mM}$ EDTA, $25 \mathrm{mM}$ Tris adjusted with $\mathrm{HCl}$ to $\mathrm{pH}$ 8.0; Add 0.4 g lysozyme per $200 \mu \mathrm{L}$ BD I for working solution $400 \mathrm{mM} \mathrm{NaOH}$ and 2\% SDS freshly mixed 1:1 $3 \mathrm{M}$ potassium acetate, $5.4 \%(\mathrm{v} / \mathrm{v})$ formic acid 
Lysis buffer:

(S. macrospora gDNA)
$10 \mathrm{mM}$ Tris adjusted with $\mathrm{HCl}$, pH 8.0, 1 mM EDTA, $100 \mathrm{mM}$

$\mathrm{NaCl}, 2 \%(\mathrm{w} / \mathrm{v}) \mathrm{SDS}$ (freshly prepared working solution)

\section{Southern blotting}

Prehybridization buffer:

$0.5 \mathrm{M} \mathrm{NaCl}$ solved in 4\% "blocking reagent"

Southern blot buffer I:

$0.25 \mathrm{M} \mathrm{HCl}$

Southern blot buffer II:

$0.5 \mathrm{M} \mathrm{NaOH}, 1.5 \mathrm{M} \mathrm{NaCl}$

Southern blot buffer III:

$1.5 \mathrm{M} \mathrm{NaCl} / 0.5 \mathrm{M}$ Tris base

First wash buffer:

$2 \mathrm{M}$ Urea, $150 \mathrm{mM} \mathrm{NaCl}, 1 \mathrm{mM} \mathrm{MgCl}, 50 \mathrm{mM} \mathrm{NaH}_{2} \mathrm{PO}_{4} \times \mathrm{H}_{2} \mathrm{O}(\mathrm{pH}$ 7.0), $50 \mathrm{mM}$ SDS and $0.2 \%$ blocking agent

Second wash buffer $(20 \mathrm{x}): \quad 1 \mathrm{M}$ Tris base, $2 \mathrm{M} \mathrm{NaCl}, \mathrm{pH} 10.0$

\section{Transformation of organisms}

\section{E.coli}

TB:

$10 \mathrm{mM}$ HEPES, $15 \mathrm{mM} \mathrm{CaCl}_{2} \times \mathrm{H}_{2} \mathrm{O}, 250 \mathrm{mM} \mathrm{KCl}, 55 \mathrm{mM} \mathrm{MnCl} 2 \times$ $\mathrm{H}_{2} \mathrm{O}, \mathrm{pH} 6.7$

\section{S. cerevisiae}

TE (10x):

$\operatorname{TE}(D)(10 x)$ :

Lithium acetate (10x):

LiAcTE(D) (1x):

LiAcTE(D)/PEG:

Salmon sperm (ss) DNA:

DTT solution:

Sorbitol:

\section{S. macrospora}

Protoplast buffer (PPB): $\quad 13 \mathrm{mM} \mathrm{Na}_{2} \mathrm{HPO}_{4}, 45 \mathrm{mM} \mathrm{KH}_{2} \mathrm{PO}_{4}, 600 \mathrm{mM} \mathrm{KCl}, \mathrm{pH} 6.0$

Transformation buffer (TPS): $1 \mathrm{M}$ sorbitol, $80 \mathrm{mM} \mathrm{CaCl}_{2}, \mathrm{pH} 7.4$

\section{Western blot/Protein methods}

Protein isolation

GFP-Trap lysis buffer:

10 mM Tris, pH 7.5, 150 mM NaCl; 10\% glycerol, 0.5 mM EDTA and freshly added $0.5 \%$ Nonidet $^{\oplus}$ P40, 2 mM DTT, 1 mM PMSF, 1 $x$ EDTA-free protease inhibitor cocktail (Roche)

GFP-Trap dilution buffer:
$100 \mathrm{mM}$ Tris $\mathrm{pH}$ 8.0, $10 \mathrm{mM}$ EDTA

$100 \mathrm{mM}$ Tris adjusted with $\mathrm{HCl}, 10$ mM EDTA, pH 7.2

$1 \mathrm{M}$ lithium acetate, $\mathrm{pH} 7.5$

$\operatorname{TE}(D)(10 x)$ and lithium acetate (10x) mixed 1:1 adjusted to the final volume with $\mathrm{H}_{2} \mathrm{O}_{\text {dest }}$

$50 \%(w / v)$ PEG 4000, TE(D)(10x) and lithium acetate (10x) mixed 1:1 adjusted with $\mathrm{H}_{2} \mathrm{O}_{\text {dest }}$ to the final volume

$10 \mathrm{mg} / \mathrm{mL}$ ssDNA in $1 \times \mathrm{TE}$

$1 \mathrm{M} \mathrm{DTT}$ in $\mathrm{H}_{2} \mathrm{O}$

$1 \mathrm{M}$ sorbitol in $\mathrm{H}_{2} \mathrm{O}$ 10 mM Tris, pH 7.5, 150 mM NaCl, 0.5 mM EDTA 


\section{SDS-PAGE and blotting}

Protein loading dye $(5 x)$ :

SDS-running buffer $(10 \mathrm{x})$ :

Transfer buffer:

$\operatorname{TBS}(10 \mathrm{x})$ :

TBST (1x)

Blocking reagent:

Immunolabelling solution:
125 mM Tris, pH 6.8, 5\% (v/v) $\beta$-mercaptoethanol, 50\% (v/v) glycerol $0.01 \%(w / v)$, bromphenol blue, $2 \%(w / v)$ SDS

$250 \mathrm{mM}$ Tris and $2 \mathrm{M}$ glycine adjusted with $\mathrm{HCl}$ to $\mathrm{pH} \mathrm{8.3,1 \% (v/v)}$ SDS

25 mM Tris, 200 mM glycine, 20\% (v/v) methanol $100 \mathrm{mM}$ Tris adjusted with $\mathrm{HCl}$ to $\mathrm{pH} 7.5,1.5 \mathrm{M} \mathrm{NaCl}$ TBS $1 x$ freshly added $0.05 \%(v / v)$ Tween $20^{\circledR}$ Difco $^{\text {TM }}$ skim milk $5 \%(w / v)$ in $1 \times$ TBST 1x TBST with $5 \%(w / v)$ skim milk and antibody as described by the manufacturer

\section{Coomassie ${ }^{\circledR}$ brilliant blue staining/destaining}

Decolouriser I:

Coomassie stain Stock I:

Coomassie stain Stock II:

Staining solution:

Decolouriser II:
$10 \%(\mathrm{v} / \mathrm{v})$ acetic acid, $40 \%(\mathrm{v} / \mathrm{v})$ ethanol

$0.2 \%(\mathrm{w} / \mathrm{v})$ Coomassie ${ }^{\circledR}$ brilliant blue R-250 in $90 \%$ (v/v) ethanol $20 \%(\mathrm{v} / \mathrm{v})$ acetic acid

Coomassie stain stock I and stock II mixed freshly 1:1 $10 \%(\mathrm{v} / \mathrm{v})$ acetic acid, 20\% (v/v) ethanol

\section{Trypsin digest/sample preparation for LC/MS}

LC/MS sample buffer:

freshly made, $2 \%(\mathrm{v} / \mathrm{v})$ acetonitrile, $0.1 \%(\mathrm{v} / \mathrm{v})$ formic acid

\subsubsection{Media}

\section{E. coli}

Lysogeny broth (LB):

$1 \%(\mathrm{w} / \mathrm{v})$ tryptone, $0.5 \%(\mathrm{w} / \mathrm{v})$ yeast extract, $0.5 \%(\mathrm{w} / \mathrm{v}) \mathrm{NaCl}, \mathrm{pH} 7.2$. Add $1.5 \%(\mathrm{w} / \mathrm{v})$ agar-agar for solid medium; add ampicillin $(100 \mu \mathrm{g} / \mathrm{mL})$ or kanamycin $(30 \mu \mathrm{g} / \mathrm{mL})$ for selection of transformants. Super optimal both (SOB):

$2 \%(\mathrm{w} / \mathrm{v})$ tryptone, $0.5 \%(\mathrm{w} / \mathrm{v})$ yeast extract, $10 \mathrm{mM} \mathrm{NaCl}, 2.5 \mathrm{mM} \mathrm{KCl}, 10 \mathrm{mM} \mathrm{MgCl}, 10 \mathrm{mM}$ $\mathrm{MgSO}_{4}, \mathrm{pH} 7.0$

\section{S. cerevisiae}

Yeast extract peptone dextrose (YEPD):

$2 \%(\mathrm{w} / \mathrm{v})$ tryptone, $1 \%(\mathrm{w} / \mathrm{v})$ yeast extract, $2 \%(\mathrm{w} / \mathrm{v})$ glucose, $\mathrm{pH} 5.8$. Add $1.5 \%(\mathrm{w} / \mathrm{v})$ SERVA agaragar for solid medium. 
Synthetic defined (SD):

$0.17 \%(\mathrm{w} / \mathrm{v})$ Difco $^{\mathrm{TM}}$ Yeast Nitrogen Base w/o amino acids and ammonium sulfate, $2 \%(\mathrm{w} / \mathrm{v})$ glucose, $0.064 \%(\mathrm{w} / \mathrm{v}) \quad$ CSM-Ade-His-Leu-Trp-Ura $\quad(0.002 \%(\mathrm{w} / \mathrm{v}) \quad$ L-methionine, $0.005 \%(\mathrm{w} / \mathrm{v})$ L-arginine hydrochloride, $0.005 \%(\mathrm{w} / \mathrm{v}) \quad$ L-isoleucine, $0.005 \%(\mathrm{w} / \mathrm{v})$ L-lysine hydrochloride, $0.005 \%(\mathrm{w} / \mathrm{v})$ Lphenylalanine, $0.005 \%(\mathrm{w} / \mathrm{v})$ L-tryptophan, $0.005 \%(\mathrm{w} / \mathrm{v})$ L-tyrosine, $0.008 \%(\mathrm{w} / \mathrm{v})$ L-aspartic acid, $0.01 \%$ $(\mathrm{w} / \mathrm{v})$ L-leucine and L-threonine, $0.014 \%(\mathrm{w} / \mathrm{v}) \mathrm{L}$-valine), $\mathrm{pH} 5.8 ; 1.5 \%(\mathrm{w} / \mathrm{v})$ SERVA agar-agar for solid medium. For selection or $\mathrm{Y} 2 \mathrm{H}$ drop experiments prepare SD medium lacking respective amino acid(s).

\section{S. macrospora}

\section{Biomalt maize medium (BMM):}

$0.8 \%(\mathrm{w} / \mathrm{v})$ corn flour (25 g/L), pH 6.5; $8 \mathrm{~g} / \mathrm{L}$ malt extract; add 1.5\% (w/v) agar-agar for solid medium; selection of transformants with Nourseothricin $(50 \mu \mathrm{g} / \mathrm{mL})$ or Hygromycin $\mathrm{B}(110 \mathrm{U} / \mathrm{mL})$; For sporulation induction add $0.5 \%(\mathrm{w} / \mathrm{v})$ sodium acetate.

\section{Complex rich medium with $10.8 \%$ sucrose (CMS):}

$1 \%(\mathrm{w} / \mathrm{v})$ glucose, $0.2 \%(\mathrm{w} / \mathrm{v})$ tryptone, $0.2 \%(\mathrm{w} / \mathrm{v})$ yeast extract, $10.8 \%(\mathrm{w} / \mathrm{v})$ sucrose, $0.15 \%(\mathrm{w} / \mathrm{v})$ $\mathrm{KH}_{2} \mathrm{PO}_{4}, 0.05 \%(\mathrm{w} / \mathrm{v}) \mathrm{KCl}, 0.05 \%(\mathrm{w} / \mathrm{v}) \mathrm{MgSO}_{4}$ heptahydrate, $0.37 \%(\mathrm{w} / \mathrm{v}) \mathrm{NH}_{4} \mathrm{Cl}$, trace elements $(10$ $\mathrm{mg} / \mathrm{LnSO}_{4}, 10 \mathrm{mg} / \mathrm{L} \mathrm{Fe}(\mathrm{II}) \mathrm{Cl}_{2}, 10 \mathrm{mg} / \mathrm{L} \mathrm{MnCl}_{2}$ ), $\mathrm{pH}$ 6.5; Add 2\% (w/v) agar-agar for solid medium.

\section{Sordaria Westergaards medium (SWG):}

$1 \times$ Westergaard solution, $2 \%(\mathrm{w} / \mathrm{v})$ glucose, $0.1 \%(\mathrm{w} / \mathrm{v})$ arginine, $0.1 \%(\mathrm{v} / \mathrm{v})$ biotin, $\mathrm{pH} 6.5 ;$ Add 1.5\% $(\mathrm{w} / \mathrm{v})$ agar-agar for solid medium.

\section{Minimal medium starch (MMS):}

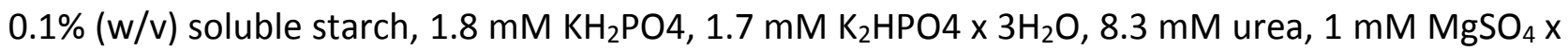
$7 \mathrm{H}_{2} \mathrm{O}, 100 \mu \mathrm{L}$ trace elements solution 2, $0.006 \mathrm{mg} / \mathrm{mL}$ biotin, $\mathrm{pH}$ 6.5; Add 2\% (w/v) agar-agar for solid medium.

\section{Prep agar:}

5\% Agar-agar in $\mathrm{H}_{2} \mathrm{O}$

\section{Top-agar:}

$0.8 \mathrm{M} \mathrm{NaCl}, 0.8 \%$ (w/v) agar-agar, $250 \mu \mathrm{g} / \mathrm{mL}$ Nourseothricin, $550 \mathrm{U} / \mathrm{mL}$ Hygromycin B.

\subsection{Methods}

\subsubsection{Cultivation of microorganisms}

\section{E. coli}

E. coli strains were grown in liquid LB and $200 \mathrm{rpm}$ or on solid LB medium at $37^{\circ} \mathrm{C}$ over night (Sambrook \& Russell, 2001). The selection was performed on LB plates supplemented with ampicillin $(100 \mu \mathrm{g} / \mathrm{mL})$ or kanamycin $(30 \mu \mathrm{g} / \mathrm{mL})$. E. coli strains for preparation of competent cells were grown in liquid SOB medium at $20^{\circ} \mathrm{C}$ and $50 \mathrm{rpm}$ overnight. 


\section{S. cerevisiae}

S. cerevisiae PJ69-4A was grown at $30{ }^{\circ} \mathrm{C}$ on solid or in liquid YEPD or SD medium at $120 \mathrm{rpm}$ overnight. Transformants were selected on solid SD medium supplemented with appropriate amino acids.

\section{S. macrospora}

S. macrospora strains were grown at $27^{\circ} \mathrm{C}$ on liquid or solid CMS, BMM, SWG or MMS with continuous light (Elleuche \& Pöggeler, 2008, Esser, 1982, Nowrousian et al., 1999, Rech et al., 2007). The vegetative growth velocity was documented in $30 \mathrm{~cm}$ race tubes filled with $25 \mathrm{~mL}$ solid SWG medium. Each race tube was inoculated with a mycelia plug of $0.5 \mathrm{~cm}$ in diameter (Nolting \& Pöggeler, 2006). The distance covered by the growing mycelium was measure every 24 hours with a ruler over 7 days (Nowrousian \& Cebula, 2005). The average vegetative growth velocity was calculated from 3 independent experiments, each performed in triplicates $(n=3)$.

Analyses of $S$. macrospora strains under stress conditions were preformed on SWG medium containing, $0.01 \% \mathrm{H}_{2} \mathrm{O}_{2}, 0.1 \mathrm{M} \mathrm{NaCl}, 0.01 \mathrm{M} \mathrm{CaCl}_{2}, 0.4 \mathrm{M}$ sorbitol, $0.003 \% \mathrm{SDS}, 100 \mu \mathrm{g} / \mathrm{mL} \mathrm{CFW}$ or on SWG with $0.6 \mathrm{M}$ glucose or without $\mathrm{KNO}_{3}$. A mycelia plug of $0.5 \mathrm{~cm}$ in diameter was placed on top of the stress inducing media and strains were grown for 7 days, at $27^{\circ} \mathrm{C}$ under continuous light.

\subsubsection{Preparation of competent microorganisms and transformation procedure}

\section{E. coli}

E. coli cells were made competent for cloning and propagation of recombinant plasmids like described by Hanahan et al. (1991). E. coli cells were grown in liquid SOB medium to an optical density $\left(O D_{600}\right)$ of 0.6 . Cells were incubated on ice for $10 \mathrm{~min}$, centrifuged at $2500 \mathrm{~g}\left(4^{\circ} \mathrm{C}\right)$, resuspended in $16 \mathrm{~mL}$ TB and centrifuged again for $10 \mathrm{~min}$ at $2500 \mathrm{~g}\left(4^{\circ} \mathrm{C}\right)$. The pellet was resuspended in $4 \mathrm{~mL}$ TB and $280 \mu \mathrm{L}$ DMSO. After 10 min incubation, chemically competent cells were immediately frozen in $100 \mu \mathrm{L}$ aliquots in liquid nitrogen and stored at $-80^{\circ} \mathrm{C}$. Chemically competent $E$. coli cells were thawed on ice and combined with plasmid DNA. After 20 min incubation on ice, cells were heat shocked for $1 \mathrm{~min}$ at $42{ }^{\circ} \mathrm{C}$, placed for $2 \mathrm{~min}$ on ice and allowed to recover in $600 \mu \mathrm{L}$ liquid LB at $37^{\circ} \mathrm{C}$ for $45 \mathrm{~min}$ at $180 \mathrm{rpm}$ (Sambrook \& Russell, 2001). Selection was performed on LB supplemented with the appropriate antibiotic.

\section{S. cerevisiae}

Homologous recombination was used to generate recombinant plasmids in $S$. cerevisiae strain PJ69-4A (James et al., 1996, Colot et al., 2006). Yeast cells were made electrocompetent as follows. A $50 \mathrm{~mL}$ S. cerevisiae cell culture grown in YEPD $\left(\mathrm{OD}_{600}\right.$ of $\left.0.8-1.2\right)$ was pelleted at $3000 \mathrm{~g}$ for $5 \mathrm{~min}$, resuspended in $1 \times \mathrm{LiAC} / \mathrm{TE}(\mathrm{D})$ and incubated for $45 \mathrm{~min}$ at $30^{\circ} \mathrm{C}$ and $100 \mathrm{rpm}$. DTT $(0.5 \mathrm{~mL}$ of a 1 M DTT stock) was added, followed by further 10 min incubation at $30^{\circ} \mathrm{C}$ at $100 \mathrm{rpm}$. Yeast cells were pelleted, washed once with water and once with $1 \mathrm{M}$ sorbitol and stored in $1 \mathrm{M}$ sorbitol (40 


\section{Materials and Methods}

$\mu \mathrm{L}$ aliquots) until transformation was performed. For the transformation $500 \mathrm{ng}$ DNA was added to the cells in $0.2 \mathrm{~cm}$ electroporation cuvettes, followed by $10 \mathrm{~min}$ incubation on ice. Immediately after electroporation, using an Eppendorf Electroporator 2510 (Eppendorf, Hamburg, Germany) at $1.5 \mathrm{kV}$ (Becker \& Lundblad, 2001), $800 \mu \mathrm{L}$ of $1 \mathrm{M}$ sorbitol was added and yeast cells dependent on the plasmid backbone used were selected for uracil, leucine or tryptophan prototrophy.

For Yeast two-hybrid ( $\mathrm{Y} 2 \mathrm{H}$ ) experiments $A D$ and $B D$ plasmids were cotransformed into chemical competent S. cerevisiae PJ69-4A (Gietz \& Schiestl, 2007). The yeast cells (10 mL for 2 transformation samples) were grown to an $\mathrm{OD}_{600}$ of $0.6-0.8$, pelleted at $3000 \mathrm{~g}$ for $5 \mathrm{~min}$, resuspended in $10 \mathrm{~mL} 1 \times$ LiOACTE(D) and shaken gently. Cells were pelleted again, the supernatant was removed and yeast cells were resuspended in a total volume of $400 \mu \mathrm{L}$ LiOACTE(D). For transformation $200 \mu \mathrm{L}$ of yeast cells were combined with ssDNA ( $1 \mathrm{mg} / \mathrm{mL}$ ), 200-800 ng Plasmid DNA and $800 \mu \mathrm{L} 50 \%$ PEG4000 (w/v) in LiOAcTE(D). The samples were vortexed and incubated for $30 \mathrm{~min}$ at $30^{\circ} \mathrm{C}$ and occasionally shaken gently. In the next step, yeast cells were incubated for 25 $\mathrm{min}$ at $42{ }^{\circ} \mathrm{C}$ and pelleted for $30 \mathrm{sec}$ at $7000 \mathrm{~g}$. The supernatant was removed and cells were resuspended in $1 \mathrm{~mL}$ YEPD medium. After recovery $\left(1 \mathrm{~h}\right.$ at $\left.30^{\circ} \mathrm{C}\right)$ under continuous shaking, yeast cells were selected for leucine and tryptophan prototrophy.

\section{Sordaria macrospora}

S. macrospora protoplasts were generated (Pöggeler et al., 1997) and transformation with recombinant plasmids was performed as described previously (Engh et al., 2007, Walz \& Kück, 1995). S. macrospora mycelium was grown in liquid cultures for 3 days, harvested and transferred to PPB containing $20 \mathrm{mg} / \mathrm{mL}$ Natuzym. After $3 \mathrm{~h}$ incubation at $27^{\circ} \mathrm{C}$ and continuous shaking at 100 rpm protoplasts were harvested with the help of a frit. Protoplasts were washed twice once with $10 \mathrm{~mL}$ PPB and second with $5 \mathrm{~mL}$ PPB. Centrifugation was performed for $4 \mathrm{~min}$ at $4000 \mathrm{~g}$ at room temperature and protoplasts were resuspended in $100 \mu \mathrm{L}$ TPS. Each transformation sample with a volume of $100 \mu \mathrm{L}$ was combined with $20 \mu \mathrm{g}$ plasmid DNA and incubated for 10 min on ice. Subsequently, 25\% (w/v) PEG6000 in TPS was added and the samples were gently mixed. After 20 minutes incubation at room temperature the protoplast solution was spread on CMS plates and incubated at $27^{\circ} \mathrm{C}$ for 24 hours. CMS plates with regenerated protoplasts were covered with $9 \mathrm{~mL}$ top-agar containing the appropriate antibiotic(s). Selection of transformants was performed on BMM media containing nourseothricin $(50 \mu \mathrm{g} / \mathrm{mL})$ and/or hygromycin $\mathrm{B}(110 \mathrm{U} / \mathrm{mL})$.

\subsubsection{Generation of S. macrospora deletion strains}

\subsubsection{Generation of $\boldsymbol{S}$. macrospora single deletion strains}

The plasmids pKOsci1hyg or pKOsci1nat encoding the deletion cassettes for generation of the S. macrospora strains $\Delta s c i 1_{\text {hyg }}$ and $\Delta s c i 1_{\text {nat }}$ were constructed by homologous recombination (1.6.2) in S. cerevisiae (Colot et al., 2006). The primer pair rud3_5'f/rud3_5'r amplified the 5' flanking region of sci1 (899 bp). For amplification of the sci1 3' flank, the primer pair rud3_3'f/rud3_3'r (637 bp) (overhang to the $h p h$ resistance cassette) or the primer pair Sci1_3'f_nat/ rud3_3'r, (637) bp 
(overhang to the nat resistance cassette) were used. The flanking regions were amplified from S. macrospora genomic DNA and contained an overhang of $29 \mathrm{bp}$ to the pRS426 vector (Christianson et al., 1992) and to the hph or nat cassette. The resistance cassette containing the $\operatorname{trpC}$ promoter sequence of $A$. nidulans fused to the $h p h$ resistance or nat resistance gene was amplified with the primer pair hph-f/hph-r from the plasmid pCB1003 or with the primer pair hph$\mathrm{f} /$ nat-1r from the plasmid pRSnat, respectively. The $h p h$ cassette had a size of $1385 \mathrm{bp}$ and the nat cassette was $929 \mathrm{bp}$ in size.

The fragments of the scil flanking regions and the respective resistance cassettes were cotransformed with a Xhol linearized pRS426 vector into the S. cerevisiae strain PJ69-4A (James et al., 1996) for homologous recombination (Mayrhofer et al., 2006) (1.6.2). Selection was performed on SD-Ura medium. The recombinant plasmids pKOsci1hyg or pKOsci1nat were isolated from yeast and transformed into $E$. coli (1.6.2). Positive clones were selected on LB medium supplemented with ampicillin. Plasmids were isolated from E. coli (1.6.8.5) and verified by sequencing (1.6.8.10). The deletion cassettes were amplified from pKOscilhyg or pKOscilnat with the primer pair rud3_5'f/rud3_3'r. The sci1 open-reading frame (ORF) in the nourseothricin resistant S. macrospora strain $\Delta \mathrm{ku} 70$ (S66001) was replace by the hph cassette (2.2.2). The sci1 ORF in the hygromycin resistant S. macrospora strain $\Delta$ ku80::hph (Herzog and Pöggeler, unpublished) was replace by the nat cassette. To eliminate the $\Delta k u 70$ or the $\Delta k u 80$ background hygromycin/nourseothricin resistance strains were crossed against the fus1-1 (S84595) (Nowrousian et al., 2012) strain and single spore isolates (ssi) resistant to hygromycin ( $\Delta$ scil $_{\text {hyg }}$ ) or nourseothricin $\left(\Delta s c i 1_{\text {nat }}\right)$ were selected on BMM supplemented with sodium acetate (1.5.9). For the $\Delta$ sci $1_{\text {hyg }}$ strain also brown single spore isolates were selected and the strain was named fus $\Delta$ sci $1_{\text {hyg. }}$. Strains with sci1 deletion ( $\Delta$ sci $1_{\text {hyg }}$ or $\Delta$ sci $1_{\text {nat }}$ ) were tested by PCR and by Southern blot (Southern, 1975) (1.6.8.1, 1.6.8.7, 1.6.8.8).

\subsubsection{Generation of $S$. macrospora double deletion strains}

The $\Delta$ sci 1 strains and the $\Delta$ pro11 strain are hyphal fusion defective. To obtain a double deletion fus $\Delta$ sci1 hyg was transformed with a plasmid construct $p 5$ 'sci1gfp (1.6.4.1) encoding the endogenous promoter of scil and the scil gene fused to egfp-trpC. Hygromycin and nourseothricin resistant strains were selected, which complemented the sterile $\Delta$ sci $1_{\text {hyg }}$ phenotype. The fertile fus $\Delta$ sci $1_{\text {hyg }}:: 5$ 'scilegfp strain was crossed to the sterile $\Delta$ pro11 strain and hygromycin resistant and nourseothricin sensitive single spores were selected. The double deletion strain $\Delta$ sci $1_{\text {hyg }} \Delta$ pro11 was verified by PCR (1.6.8.1). 


\subsubsection{Generation of sci1 expression plasmids for transformation into S. macrospora}

Expression plasmids were generated to synthesize C-terminally eGFP- or DsRED full-length SCI1 proteins in S. macrospora. Also, constructs containing scil fragments were generated to express fragments of sci1 in the $\Delta$ sci $1_{\text {hyg }}$ strain.

\subsubsection{Generation of sci1 full length constructs for transformation into S. macrospora}

Sci1-egfp or sci1-Dsred fusion gene constructs under the control of the endogenous sci1 promotor were generated. The $5^{\prime}$ flanking region of scil containing the endogenous promoter of scil and the sci1 ORF were amplified with the primer pair rud3_5'f/scilgfp_r (1875 bp) from S. macrospora gDNA. The primers had $29 \mathrm{bp}$ overhangs to the pRSnat plasmid and to the egfp sequence, respectively. The egfp sequence fused to the $\operatorname{trp} C$ terminator sequence of $A$. nidulans was amplified from the plasmid p1783-1 with the primer pair GFP-f/pRS426GFPrev (1510 bp) generating an 29 bp overhang to pRSnat. Homologous recombination of both fragments with Xhol hydrolyzed pRSnat was performed in the yeast PJ69-4A (1.6.2) to generate the plasmid p5'sci1gfp.

A further construct was constructed in the pRSnat background containing the $5^{\prime}$ flanking region of sci1 and the scil gene fused to the Dsred sequence. The 5' flanking region together with the sci1 ORF was amplified with the primer pair rud3_5'f/sci1dsRed_r (1875 bp) using S. macrospora gDNA as template. The Dsred sequence fused to the $\operatorname{trp} C$ terminator sequence of $A$. nidulans was amplified with the primer pair DsRed-f/pRS426GFPrev (1466 bp) from pRHN1. Both fragments were homologously recombined (1.6.2) with the Xhol hydrolyzed pRSnat vector generating plasmid p5'sci1Dsred.

Also, sci1-egfp or sci1-Dsred fusion gene constructs under the control of the ccg1 promoter of $N$. crassa were created. The ccg1 promoter sequence was amplified from pHAN1 with the primer pair pRSccg1/Pccg1-r generating a 29 bp overhang to pRSnat. The sci1 ORF was amplified with the primer pair sci1ccg1_f/sci1gfp_ror with sci1ccg1_f/sci1dsRed_r from S. macrospora gDNA and had overhangs to the ccg1 promoter sequence and to the egfp or to the Dsred sequence, respectively. The egfp fused to the $\operatorname{trp} C$ terminator of $A$. nidulans was amplified using the plasmid p1783-1 (1510 bp) and Dsred fused to trpC was amplified from pRHN1 with the primer pairs GFP-f/pRS426GFPrev and DsRed-f/pRS426GFPrev (1466 bp), respectively producing 29 bp overhangs to pRSnat. The respective fragments were homologously recombined with the Xhol hydrolyzed pRSnat vector in the yeast PJ69-4A (1.6.2).

The obtained plasmids psci1gfp and psci1Dsred were transformed into E.coli (1.6.8.5) and were verified by sequencing (1.6.8.10). The respective plasmids were transformed into $\Delta$ sci $1_{\text {hyg }}(2.2 .2)$ and hygromycin and nourseothricin resistant strains were selected. The strains, $\Delta$ sci $1_{\text {hyg }}:: 5^{\prime}$ scilegfp,

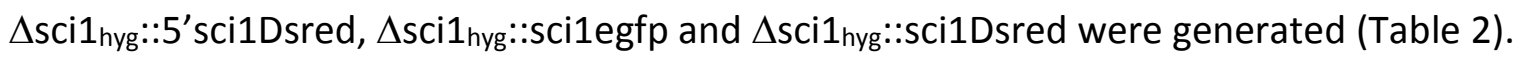

\subsubsection{Construction of plasmids containing fragments of sci1 and transformation into S. macrospora}

Plasmids containing sci1 fragments were generated for complementation studies in the sterile $\Delta s c i 1_{\text {hyg }}$ background. All fragments were set under the control of the endogenous sci1 promoter 
and were fused to the egfp-trpC sequence. The endogenous promoter of scil was amplified from p5'sci1gfp using the primer pair rud3_5'f/5'sci1_rev and had an 29 bp overhang to the pRSnat plasmid. The sci1 fragments were amplified from p5'sci1gfp with the primer pairs shown in table 6 generating $29 \mathrm{bp}$ overhangs to the 5' flanking region of scil and to the egfp sequence.

The egfp-trpC sequence (1510 bp) was amplified from p1783-1 with the primer pair GFP$\mathrm{f} / \mathrm{pRS} 426 \mathrm{GFPrev}$ containing an $29 \mathrm{bp}$ overhang to $\mathrm{pRS}$ nat. All three fragments, the 5 ' flanking region of sci1, the respective sci1 fragment the egfp-trpC sequence and the Xhol hydrolyzed plasmid vector were cotransformed into PJ69-4A. The plasmids psci163-207_nat, psci1121-207_nat, psci1 121 298_nat and psci1208-298_nat (Table 3, Table 6) were generated by homologous recombination in PJ69-4A (1.6.2).

Table 6: Overview of primer pairs used to amplify fragments of sci1 and pRSnat plasmids generated

\begin{tabular}{|c|c|c|c|c|c|}
\hline $\begin{array}{l}\text { Forward } \\
\text { primer }\end{array}$ & $\begin{array}{l}\text { Reverse } \\
\text { primer }\end{array}$ & $\begin{array}{l}\text { Amplified sci1 } \\
\text { fragment }\end{array}$ & $\begin{array}{l}\text { Fragment } \\
\text { size in bp }\end{array}$ & $\begin{array}{l}\text { Plasmid backbone / } \\
\text { enzyme used for } \\
\text { hydrolysis }\end{array}$ & Constructed plasmid \\
\hline sci1_t1f & sci1_t7r & $\operatorname{sci}_{63-207}$ & 437 & pRSnat/Xhol & psci163-207_nat \\
\hline sci1_t3f & sci1_t7r & $\operatorname{sci}_{121-207}$ & 260 & pRSnat/ Xhol & psci1121-207_nat \\
\hline sci1_t3f & scilgfp_r & sci1 $_{121-298}$ & 533 & pRSnat/ Xhol & psci1121-298_nat \\
\hline sci1_t5f & sci1gfp_r & sci1 $_{208-298}$ & 272 & pRSnat/ Xhol & psci1 208-298_nat \\
\hline
\end{tabular}

The numbers given in the amplified fragment represent the amino acids of scil encoded in the generated fragments

Plasmids were transformed into $E$. coli (1.6.8.5) and verified by sequencing (1.6.8.10) before they were used to transform $\Delta$ sci $1_{\text {hyg. }}$ S. macrospora strains were transformed (2.2.2) with plasmids containing scil fragments and hygromycin and nourseothricin clones were selected. The generated strains are listed in table 2.

\subsubsection{Transformation of wt S. macrospora strains with pHA_nat and pFLAG_nat}

The $c c g 1$ sequence fused to the $h a$-sequence ( $981 \mathrm{bp}$ ) was amplified from the plasmid pHAN-1 with the primer pair pRSccg1/Pccg1HAr_TtrpC containing 29 bp overhangs to the pRSnat vector and to the $\operatorname{trpC}$-sequence. The terminator sequence $\operatorname{trpC}$ (768 bp) was amplified from the plasmid p17831 with the primer pair TrpC_F/pRS426GFPrev containing a 29 bp overhang to the pRSnat plasmid. Both amplicons were cotransformed with the Xhol hydrolyzed pRSnat plasmid into the yeast strain PJ69-4A for homologous recombination (1.6.2).

The ccg1-flag-sequence (1017 bp) was amplified from the plasmid pFLAGN1 using the primer pair pRSccg1/Pccg1FLAGr_TtrpC. The fragment contained an $29 \mathrm{bp}$ overhang to the pRSnat vector and the $\operatorname{trpC}$-sequence. The $\operatorname{trpC}$ sequence (768 bp) amplified from plasmid p1783-1 with the primer pair TrpC_F/pRS426GFPrev contained an overhang (29 bp) to the pRSnat plasmid. The two fragments and the Xhol hydrolyzed pRSnat plasmid were homologously recombined in PJ69-4A. 
The successfully generated plasmid pHA_nat or pFLAG_nat were transformed into wild type S. macrospora strain (2.2.2) and nourseothricin resistant strains wt::ha or wt::flag were selected, respectively. The strains were used as negative controls for HA- or FLAG pull-downs coupled to LC/MS (Table 8).

\subsubsection{Generation of expression plasmids for complementation attempts of $S$. macrospora with the mammalian FGFR1OP2 and yeast Far3 and Far7}

Lentivirus-derived plasmids pRRLsinhCMV-IG containing Rat Fgfr1op2 (644 bp), human wt FGFR1OP2 (758 bp) and human single nucleotide polymorphism 1 (SNP1) FGFR1OP2 (FGFR1OP2_SNP1) (758 bp) genes derived from cDNA were kindly donated by Prof. D.M.D. Ichiro Nishimura (Lin et al., 2010). The mammalian FGFR1OP2 genes are highly conserved and genes from human and rat were amplified with the primer pair FG_RNf/FG_RNr. The ccg1 promoter (921 bp) was amplified from pHAN1 with the primer pair Pccg1/ccg1_FGRN containing overhangs to the pRSnat vector and the FGFR1OP2 genes.

The $h a$-tag sequence fused to the $\operatorname{trpC}$ terminator (819 bp) was amplified with the primer pair HAf_FGRN/pRS426GFPrev from plasmid pHA_nat and contained 29 bp overhangs to the FGFR1OP2 genes and the pRSnat plasmid. The fragments pccg1, FGFR1OP2s and HA-trpC were homologously recombined with the Xhol hydrolyzed pRSnat vector in PJ69-4A (1.6.2) and three plasmids pPcFGwtRN, pPcFGwtHsHs, pPcFGSNP1 were generated (Table 3), transformed into E. coli and sequenced $(1.6 .8 .5,1.6 .8 .10)$. The plasmids pPcFGwtRN, pPcFGwtHsHs, pPcFGSNP1 were isolated from $E$. coli and transformed into $\Delta$ sci $_{\text {hyg }}$ (2.2.2) generating the nourseothricin and hygromycin

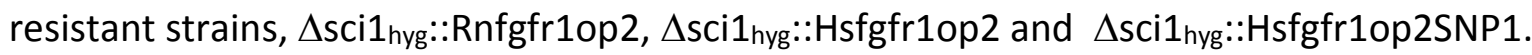

S. cerevisiae FAR3 and FAR7 genes were amplified with the primer pairs Far3_f/Far3_r (662 bp) and Far7_f/Far7flag_r (622 bp) with overhangs to ccg1 and ha-trpC or flag-trpC, respectively. S. cerevisiae PJ69-4A genomic DNA was used as template. The ha-trpC or flag-trpC sequence were amplified from the plasmids pHA_nat or pFLAG_hyg, using the primer pairs HAf/pRS426GFPrev (819 bp) or FLAGf/pRS426GFPrev (852 bp), respectively containing an overhang to pRSnat. Homologous recombination of ccg1, FAR3 or FAR7, HA-trpc or FLAG-trpC, respectively and Xhol hydrolyzed pRSnat was performed in PJ69-4A (2.2.2) and the plasmids pFar3HA and pFar7FLAG were generated. The $\Delta$ sci1 $1_{\text {hyg }}$ strain was transformed (2.2.2) with pFar3HA or pFar7FLAG and

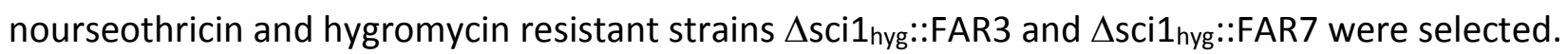

\subsubsection{Generation of $S$. macrospora strains expressing fluorescent fusion proteins}

\subsubsection{Complementation of the $\Delta \mathrm{sci} 1_{\mathrm{hyg}} \Delta$ pro11 strain}

The $\Delta$ sci $1_{\text {hyg }} \Delta$ pro11 strain was cotransformed with the plasmids psci1Dsred and pHAPRO11GFP (2.2.2) and nourseothricin and hygromycin resistant strains were selected. The resulting strain $\Delta$ sci1 $1_{\text {hyg }} \Delta$ pro11::sci1Dsred+hapro11egfp became fertile indicating that both constructs were functional. 
1.6.7.2 Generation of S. macrospora strains for analysis of nuclei, mitochondria, ER, actin, nuclear envelope as well as early and late endosomes with fluorescence microscopy

For generation of the plasmid pRH2B_nat, the $h 2 b$-tdTomato construct under the control of the gpd promoter and the trpC terminator of $A$. nidulans were amplified with the primer pair pRS426GFPrev/ pRS426GPDf2 (3785 bp) from pRH2B. The fragment was homologously recombined (2.2.2) with the Xhol hydrolyzed pRSnat plasmid in the yeast PJ69-4A. The pRH2B_nat was transformed into $E$. coli and verified by sequencing $(2.2 .8 .5,2.2 .8 .10)$.

The wt S. macrospora strain and the $\Delta$ sci $1_{\text {hyg }}$ strain each were transformed with the plasmids pRH2B_nat (nuclei) or pMito-DsRED (mitochondria), or pAL-lifeact (actin) or pDsREDKDEL (ER) and nourseothricin or hygromycin and nourseothricin resistant strains, respectively were selected (2.2.2). Also, wt S. macrospora and $\Delta$ sci $_{\text {nat }}$ were transformed with pHEgfpRab5 (early endosomes) and pHEgfpRab7 (late endosomes) and hygromycin as well was hygromycin and nourseothricin resistant strains, respectively were selected (2.2.2). The strains generated and used for fluorescence microscopy are listed in table 2.

\subsubsection{Generation of SmPOM152 expression plasmid for transformation into S. macrospora} The Smpom152 gene (SMAC_03501) was amplified from genomic S. macrospora DNA with the primer pair Pom_ccgf/Pom_gfpr (3916 bp) and contained 29 bp overhangs to the ccg1 promoter. The ccg1 promoter (921 bp) was amplified with the primer pair pRSccg1/ccg1-r from pHAN-1 containing an $29 \mathrm{bp}$ overhang to egfp. The egfp-trpC fragment (1510 bp) was amplified from p17831 containing an overhang (29 bp) to the pRSnat plasmid. All generated fragments were cotransformed into PJ69-4A for homologous recombination (2.2.2) with Xhol hydrolyzed pRSnat and the plasmid pSmPOM152 was generated. The plasmid pSmPOM152 was transformed into $E$. coli and verified by sequencing $(2.2 .8 .5,2.2 .8 .10)$. Finally, pSmPOM152 was transformed into the wt and into $\Delta$ sci $_{\text {hyg }}(2.2 .2)$ and nourseothricin and nourseothricin/hygromycin resistant strains were selected generating the strains wt::Smpom152egfp and $\Delta$ sci1 $_{\text {hyg }}:$ Smpom152egfp (Table 2).

\subsubsection{Generation of $\Delta$ pro11::hapro11egfp strain}

The S. macrospora $\Delta$ pro11 strain was transformed (2.2.2) with the pHAPRO11GFP plasmid encoding the pro11 gene under the control of the $c c g 1$ promoter and the $\operatorname{trp} C$ terminator and fused to the ha and egfp sequence. Hygromycin and nourseothricin resistant strains were selected and complemented the sterile phenotype indicating that the hapro1legfp construct is functional generating the strain $\Delta$ pro11::hapro11egfp (Table 2).

\subsubsection{Molecular biology methods: nucleic acids}

\subsubsection{PCR}

DNA fragments were amplified from gDNA, complementary DNA or plasmids using polymerase chain reaction (PCR) (Mullis \& Fallona, 1987). For the PCR reaction 50-100 ng of DNA were used. $E$. coli colony PCR was performed in volumes of $12.5 \mu \mathrm{L}$ using the $E$. coli culture as DNA source. The 
PCR was performed as suggested by the enzyme manufacturer. The melting temperature of the primers was adjusted as required for the oligonucleotides. PCR samples were shortly stored at $4^{\circ} \mathrm{C}$ or for long term storage at $-20^{\circ} \mathrm{C}$. Components used for the standard $50 \mu \mathrm{L}$ PCR reactions were as follows:

\section{Phusion Polymerase}

$\begin{array}{ll}\text { Sterile } \mathrm{H}_{2} \mathrm{O}_{\text {dest }} & \text { up to } 50 \mu \mathrm{L} \\ 5 \times \text { HF-buffer } & 10 \mu \mathrm{L} \\ 10 \mathrm{mM} \text { dNTPs } & 1 \mu \mathrm{L} \\ 10 \text { pM Primer 1 } & 1 \mu \mathrm{L} \\ 10 \text { pM Primer 2 } & 1 \mu \mathrm{L} \\ \text { Template DNA } & 50-100 \mathrm{ng} \\ \text { DMSO } & 1.5 \mu \mathrm{L} \\ \text { Phu Polymerase } & 0.5 \mu \mathrm{L}\end{array}$

\section{TopTaq Polymerase}

$\begin{array}{ll}\text { Sterile } \mathrm{H}_{2} \mathrm{O}_{\text {dest }} & \text { up to } 50 \mu \mathrm{L} \\ 10 \text { x TopTaq PCR buffer } & 5 \mu \mathrm{L} \\ 10 \mathrm{mM} \text { dNTPs } & 1 \mu \mathrm{L} \\ 10 \text { pM Primer 1 } & 1 \mu \mathrm{L} \\ 10 \text { pM Primer 2 } & 1 \mu \mathrm{L} \\ \text { Template DNA } & <1 \mu \mathrm{g} / \text { reaction } \\ \text { TopTaq } & 0.25 \mu \mathrm{L}\end{array}$

\subsubsection{Separation of nucleic acids with agarose-gel electrophoresis}

DNA fragments were mixed with $6 x$ loading dye loaded on $1 \%$ or $2 \% 1 \times$ TBE agarose gels and separated in a horizontal Mupid one (Biozym Scientific GmbH, Germany) gel electrophoresis chamber in 0.5\% TBE buffer applying 100-130 V (Sambrook et al., 2001).

The GeneRuler ${ }^{T M}$ DNA Ladder Mix (Thermo Scientific, SM0331) or the GeneRuler ${ }^{T M} 1 \mathrm{~kb}$ DNA Ladder (Thermo Scientific, SM0313) were used as marker for DNA fragment size. The DNA was stained for 10-20 $\mathrm{min}$ in a $1 \mathrm{\mu g} / \mathrm{mL}$ ethidium bromide solution.

RNA samples were mixed with 2x RNA buffer followed by incubation for $10 \mathrm{~min}$ at $65^{\circ} \mathrm{C}$. Ribonucleic acids were separated in an agarose gel containing 1.2\% 1x MOPS and 5\% formaldehyde and immersed in $1 \times$ MOPS buffer at $80-100 \mathrm{~V}$ in a gel electrophoresis chamber. The GeneRuler ${ }^{\mathrm{TM}} 1 \mathrm{~kb}$ DNA Ladder (Thermo Scientific, SM0313) was used as marker for RNA fragment size.

DNA and RNA were visualized by UV light and documented with the BioDocAnalyzer (Biometra, Germany).

\subsubsection{Purification of nucleic acids}

\section{Isolation of DNA from agarose gels}

DNA from agarose gels was isolated with the "QIAquick Gel Extraction" kit as described by the manufacturer.

\section{Dialysis of aqueous solutions/purification of PCR products}

Dialysis of plasmids or amplicons for $S$. cerevisiae transformation was performed with dialysis filters. Eighteen microliter of aqueous plasmid solution were applied on the dialysis filters (Merck Millipore, Germany) placed onto sterile $\mathrm{H}_{2} \mathrm{O}_{\text {dest }}$ at room temperature and were removed after 20 min. PCR products were alternatively purified with the "QIAquick PCR purification kit" as described by the manufacturer. 


\subsubsection{Hydrolysis and ligation of nucleic acids}

\section{Hydrolysis of plasmids}

Hydrolysis of plasmids was performed with $1 \mathrm{U}$ restriction endonuclease per $\mu \mathrm{g}$ of DNA in 10-20 $\mu \mathrm{L}$ volumes $\left(37^{\circ} \mathrm{C}\right.$ for 2 hours). Hydrolysis was performed in buffer solutions as suggested by the manufacturer.

\section{Dephosphorylation of plasmids and ligation}

Vectors for ligation were hydrolysed as described before and subsequently dephosphorylated with the "Rapid DNA Dephos \& Ligation kit" as suggested by the manufacturer and ligation was performed with the T4 DNA ligase as described by the manufacturer.

\subsubsection{Isolation of plasmid DNA from $E$. coli}

For plasmid DNA isolation mini- and midi- preparation was used applying the "FastGene ${ }^{\circledR}$ Plasmid Mini kit" as described by the manufacturer or was performed according to Birnboim and Doly (1979).

\subsubsection{Isolation of plasmid DNA from $S$. cerevisiae}

S. cerevisiae colonies on SD-Ura plates were washed down from the plates with sterile $\mathrm{H}_{2} \mathrm{O}_{\text {dest. }}$. For the isolation of plasmid DNA from S. cerevisiae the "ZR Fungal/Bacterial DNA MiniPrep ${ }^{\mathrm{TM}}$ kit" was applied as suggested by the manufacturer.

\subsubsection{Isolation of RNA and genomic DNA from S. macrospora}

For RNA and gDNA isolation, S. macrospora mycelium was grown for 3 days at $27^{\circ} \mathrm{C}$ in liquid BMM, harvested, dried by pressing and grounded to powder in a mortar with the help of liquid nitrogen.

\section{Isolation of RNA}

RNA was isolated from S. macrospora as described by Elleuche and Pöggeler (2009). One milliliter trizol was added to the pulverized mycelium followed by centrifugation for $10 \mathrm{~min}$ at 13,000 rpm. The supernatant was mixed with $200 \mu \mathrm{L}$ chloroform in a new tube followed by centrifugation for $10 \mathrm{~min}$ at 13,000 rpm. The supernatant was mixed with the same amount of ice-cold isopropanol. The RNA was incubated for $10 \mathrm{~min}$ at RT, centrifuged again and washed with $70 \%$ ice-cold ethanol. The RNA was dried under the hood, combined with $120 \mu \mathrm{L}$ water and solved at $1000 \mathrm{rpm}$ for 30 $\min$ at $60^{\circ} \mathrm{C}$.

\section{Isolation of genomic DNA (gDNA)}

E-cups ( $2 \mathrm{~mL}$ ) were filled to $1 / 3$ with nitrogen grounded mycelium and $600 \mu \mathrm{L}$ lysis buffer was added followed by vortexing and $30 \mathrm{~min}$ incubation at $70^{\circ} \mathrm{C}$. The gDNA was isolated according to the phenol-chloroform extraction method (Brawerman et al., 1972, Kirby, 1956, Pöggeler et al., 1997). Alternatively, gDNA from S. macrospora was isolated for PCR amplification using a modified sodium acetate/isopropanol protocol (Cenis, 1992). Mycelium was press dried in $2 \mathrm{~mL}$ tubes, frozen at $80^{\circ} \mathrm{C}$, supplemented with $6 \varnothing 2.85-3.45 \mathrm{~mm}$ glass beads, combined with $300 \mu \mathrm{L}$ lysis buffer and 
homogenized for $5 \mathrm{~min}$ with $30 \mathrm{~Hz}$ in the Qiagen Retsch ${ }^{\circledR}$ TissueLyser (Qiagen Retsch $\mathrm{GmbH}$, Germany). Samples were incubated for $30 \mathrm{~min}$ at $70^{\circ} \mathrm{C}, 200 \mu \mathrm{L} 3 \mathrm{M}$ sodium acetate (pH 5.2) was added followed by $10 \mathrm{~min}$ incubation at $-80^{\circ} \mathrm{C}$. Samples were centrifuged $10 \mathrm{~min}(13,000 \mathrm{rpm})$ at RT and the supernatants were combined with the same amount of isopropanol. After incubation for $10 \mathrm{~min}$ at RT, samples were again centrifuged (10 $\mathrm{min}, 13,000 \mathrm{rpm})$, the supernatant was discarded and the pellet was washed with $300 \mu \mathrm{L} 70 \%$ ethanol, followed by 2 min centrifugation at $13,000 \mathrm{rpm}$.

The gDNA was dried under the hood, dissolved in sterile $\mathrm{H}_{2} \mathrm{O}_{\text {dest }}$ and stored at $4^{\circ} \mathrm{C}$, for short-term storage or at $-20^{\circ} \mathrm{C}$, for long-term storage.

\subsubsection{Southern blotting and hybridization}

In locus-deletion of genes and insertion of a resistance cassette was verified by Southern blot (Southern, 1975). Genomic DNA (30-50 $\mu \mathrm{g}$ ) was hydrolyzed with the respective enzymes in a $50 \mu \mathrm{L}$ reaction volume. The hydrolyzed gDNA was separated on an $1 \%$ agarose gel at $100 \mathrm{~V}$. The agarose gel was washed for $10 \mathrm{~min}$ in Southern blot buffer I, $25 \mathrm{~min}$ in Southern blot buffer II and finally in Southern blot buffer III for $30 \mathrm{~min}$. The DNA was blotted for 2-3 h onto a Amersham Hybond ${ }^{\mathrm{TM}}{ }_{-} \mathrm{N}^{+}$ membrane. The membrane was dried $\left(7 \mathrm{~min} / 70^{\circ} \mathrm{C}\right)$ and DNA was cross-linked by exposure to UVlight ( 3 min each side of the membrane).

The DNA for the probe for sci1 was generated with the primer pair sosci1_f/sosci1_r (650 bp) and purified with the "QIAquick gel extraction kit". $100 \mathrm{ng}$ of the specific probe and 1 $\mu \mathrm{L}$ of 1:1000 diluted GeneRuler"M $1 \mathrm{~kb}$ DNA Ladder were labeled with the "AlkPhos Direct Labelling and Detection Kit". The detection was performed as described by the manufacturer's manual.

\subsubsection{Synthesis of cDNA and qRT-PCR}

S. macrospora wt was grown in liquid $\mathrm{BMM}$ for $3,4,5,6$ and 7 days at $27^{\circ} \mathrm{C}$, in floating cultures for sexual reproduction conditions and in shaking (130 rpm) Erlenmeyer flasks filled with $100 \mu \mathrm{L}$ BM for vegetative conditions (Nowrousian \& Cebula, 2005). RNA was isolated as described in 1.6.8.7 "Isolation of RNA". The quantitative reverse-transcription PCR was performed two times with independent biological samples in triplicates. The qRT-PCR was performed as described before and the Mastercycler ${ }^{\circledR}$ ep realplex (Eppendorf, Hamburg, Germany) was used (Elleuche \& Pöggeler, 2009). The primer pair RTsci1f/RTsci1r amplified a 236 bp from sci1. Primer combination SSU-f/SSU$r$ resulted in the amplification of a $180 \mathrm{bp}$ fragment coding for the small rRNA subunit of S. macrospora, which was used as a reference for the normalization of $\mathrm{Ct}$ values. The significance of the qRT-PCR results was calculated with the software tool RESTC (Pfaffl et al., 2002). 


\subsubsection{Synthesis of oligonucleotides and sequencing}

Oligonucleotides were synthesized by Eurofins MWG Operon (Ebersberg, Germany). The primers are presented in table 4. Sequencing of DNA was performed by the Göttinger Genom Labor (G2L) (Georg-August University of Göttingen, Germany) or by Seqlab (Sequence Laboratories Göttingen $\mathrm{GmbH}$, Germany). Sequences were analyzed with the help of the software Chromas Lite 2.01 (19982005 Technelysium Pty Ltd). The Nucleotide BLAST search tool (Altschul et al., 1990) (http://blast.ncbi.nlm.nih.gov/Blast.cgi) was used to compare the sequenced plasmid/DNA with the sequences of the plasmids or the predicted gDNA/cDNA sequences.

\subsubsection{Molecular biology methods: proteins}

\subsubsection{Isolation of proteins from $S$. macrospora}

S. macrospora mycelium was harvested, pressed dried between paper towels and ground in liquid nitrogen. One gram of mycelium was combined with $500 \mu \mathrm{L}$ GFP-Trap lysis buffer and centrifuged at $16.000 \mathrm{~g}$ for $30 \mathrm{~min}$. The supernatant was centrifuged again at $16.000 \mathrm{~g}$ for $30 \mathrm{~min}$ and the supernatant was transferred to a new tube.

Protein extracts for Western blot were combined with the appropriate amount of $5 \times$ protein loading dye or with the NuPAGETM LDS Sample Buffer $(4 \mathrm{x})$ supplemented with DTT, boiled for 10 $\min$ at $98^{\circ} \mathrm{C}$ (Laemmli, 1970) or for $10 \mathrm{~min}$ at $70^{\circ} \mathrm{C}$, respectively and $20-30 \mu \mathrm{L}$ were used for SDSPAGE.

For the pull-downs, GFP-Trap_A beads (anti-GFP $V_{H} H$ coupled to agarose beads) were used to bind eGFP fusion proteins. GFP-Trap_A beads were washed three times with $350 \mu \mathrm{L}$ ice-cold GFP-Trap dilution buffer (centrifugation $2 \mathrm{~min}, 3000 \mathrm{rpm}, 4^{\circ} \mathrm{C}$ ).

Supernatants containing FLAG fusion proteins were incubated with the respective antibody for $2 \mathrm{~h}$ on a rotation device $\left(4^{\circ}\right)$. Protein A Sepharose ${ }^{T M}$ CL-4B beads or anti-HA-agarose beads were equilibrated with GFP-Trap dilution buffer, $10 \mathrm{mg}$ beads were incubated for $30 \mathrm{~min}$ with $350 \mu \mathrm{L}$ icecold GFP-Trap dilution buffer followed by 2 washing steps (centrifugation $2 \mathrm{~min}, 3000 \mathrm{rpm}, 4^{\circ} \mathrm{C}$ ). The antibody-antigen complex was bound to Protein A Sepharose ${ }^{\mathrm{TM}} \mathrm{CL}-4 \mathrm{~B}$ for $1 \mathrm{~h}$ on a rotation device $\left(4^{\circ}\right)$.

For the pull-downs $1 \mathrm{~mL}$ of the supernatant or of the antibody-antigen complex was incubated with GFP-Trap_A, anti-HA-agarose beads or Protein A Sepharose ${ }^{\mathrm{TM}} \mathrm{CL}-4 \mathrm{~B}$ beads, on a rotating device for $3 \mathrm{~h}$ or $1 \mathrm{~h}$ at $4^{\circ} \mathrm{C}$, respectively. The beads were washed twice with $500 \mu \mathrm{L}$ GFP-Trap dilution buffer containing $1 \mathrm{mM}$ PMSF and EDTA-free protease inhibitor cocktail. Washing buffer was removed and samples were boiled with the appropriate amount of 5x protein loading dye for 10 $\min$ at $98^{\circ} \mathrm{C}$ and $20-30 \mu \mathrm{L}$ were used for SDS-PAGE.

\subsubsection{Determination of protein concentration}

Protein concentration was measured using the Bradford assay (Bradford, 1976). The protein sample $(10 \mu \mathrm{L})$ was mixed with $1 \times$ Roti $^{\circledR}$-Quant reagent $(990 \mu \mathrm{L})$ and the absorption was determined 
at $595 \mathrm{~nm}$ in a "Libra S12" (biochrom, UK) spectrophotometer in a $1 \mathrm{~mL}$ disposable cuvette after incubated for $2 \mathrm{~min}$ at RT. For generation of a standard curve bovine serum albumin was used.

\subsubsection{SDS-PAGE}

Proteins were separated according to their size by SDS-PAGE (Laemmli, 1970). With the help of "Mini-PROTEAN ${ }^{\circledR}$ Tetra Cell" (Bio-Rad, Germany) SDS-gels (Table 7) were prepared. Protein samples were mixed with the protein loading dye or with the protein loading dye or the NuPAGE ${ }^{\mathrm{TM}}$ LDS sample buffer supplemented with DTT were loaded into pockets of SDS gels immersed in SDSPAGE-running buffer. Proteins were separated with SDS-PAGE at $130 \mathrm{~V}$. Five microliter of the Pageruler $^{\mathrm{TM}}$ Prestained Protein Ladder were used as protein standard.

Table 7: Recipes for stacking and resolving polyacrylamide gels

\begin{tabular}{|llll} 
Component & Stacking Gel (5\% Acrylamide) & Resolving Gel (12\% Acrylamide) & Unit \\
\hline $\begin{array}{l}\text { Rotiphorese Gel30 } \\
\text { (Roth, Karlsruhe) }\end{array}$ & 0.250 & 2.800 & $\mathrm{ml}$ \\
\hline $\mathbf{1 . 5 M}$ Tris/HCl, pH8,0 & - & & $\mathrm{ml}$ \\
\hline $\mathbf{0 . 5 M}$ Tris/HCl, pH 6,5 & 0.375 & 1.750 & $\mathrm{ml}$ \\
\hline $\mathbf{2 0} \%(\mathbf{w} / \mathbf{v})$ SDS & 7.500 & - & $\mu \mathrm{l}$ \\
\hline $\mathrm{H}_{\mathbf{2}} \mathbf{O}_{\text {dest. }}$ & 0.860 & 37.000 & $\mathrm{ml}$ \\
\hline TEMED & 1.300 & 2.350 & $\mu \mathrm{l}$ \\
\hline $\mathbf{1 0} \%(\mathbf{w} / \mathbf{v})$ APS & 10.000 & 5.700 & $\mu \mathrm{l}$ \\
\hline
\end{tabular}

\subsubsection{Western blotting}

Western blot analysis was used to detect fusion proteins by an antigen-antibody reaction. Proteins separated in SDS-PAGE were transferred to a Amersham ${ }^{\mathrm{TM}}$ Protran $^{\mathrm{TM}}$ Nitrocellulose Blotting Membrane applying $200 \mathrm{~mA}$ a semi-dry device (Biometra, Germany) (Towbin et al., 1979). The membrane was washed once with TBST buffer for $20 \mathrm{~min}$ and blocked with $5 \%(\mathrm{w} / \mathrm{v})$ milk powder in TBST for $1 \mathrm{~h}$ at RT. Antigen-antibody reaction was performed using a horse-radish peroxidase (HRP) coupled GFP-antibody or a specific primary antibody (Table 5) solved in $5 \%$ skim milk/TBST. The membrane and the antibody solution were incubated over night at $4^{\circ} \mathrm{C}$. After the antibody was removed the membrane was washed three times for $20 \mathrm{~min}$ with TBST. If required the respective secondary antibody was applied to the membrane for $1 \mathrm{~h}$ at RT and the membrane was again washed three times with TBST. Enhanced chemiluminescent reaction was used to detect the HRP coupled antibodies using the Immobilon ${ }^{\text {TM }}$ Western HRP Substrate kit (Haan \& Behrmann, 2007).

\subsubsection{Coomassie staining of polyacrylamide gels}

Proteins resolved on SDS-Gels were stained with Coomassie ${ }^{\circledR}$ Brilliant Blue R250 (Wilson, 1979). SDS-Gels were fixed for 30 minutes in the decolorizer I solution. Coomassie stain Stock I containing 
$100 \mathrm{mg}$ Brilliant Blue R solved in $50 \mathrm{~mL}$ of $90 \%$ ethanol was mixed 1:1 with Coomassie stain Stock II and SDS-gels were stained for $20 \mathrm{~min}$ under continuous gentle agitation. Stained gels were washed for $5 \mathrm{~min}$ in the decolorizer I solution. Further, washing and removing of Coomassie ${ }^{\circledR}$ Brilliant Blue R250 was performed in several wash steps with decolorizer II solution. The SDS-gels were destained in several steps, three times for $20 \mathrm{~min}$ and over night. If required gels were again destained for 20 min and were finally washed two times (5 min) in $\mathrm{H}_{2} \mathrm{O}_{\text {dest. }}$. Each lane was cut out and further cutted into 10 pieces for the trypsin digest.

\subsubsection{Trypsin digest, stage tipping and sample preparation}

Trypsin digest was performed to prepare protein samples for LC/MS (Shevchenko et al., 1996). Ingel digestion with trypsin was performed for each SDS-Gel piece separately in $1.5 \mathrm{~mL}$ Protein LoBind Tubes.

Acetonitrile $(40 \mu \mathrm{L})$ was added to fully cover the gel pieces. Samples were incubated for $10 \mathrm{~min}$ under constant shaking, and acetonitrile was removed. Gel pieces were dried for $10 \mathrm{~min}$ in the Eppendorf concentrator 5301 (Eppendorf, Germany) and $150 \mu \mathrm{L} 10 \mathrm{mM} \mathrm{DTT} / 100 \mathrm{mM} \mathrm{NH}_{4} \mathrm{HCO}_{3}$ was added followed by incubation for $1 \mathrm{~h}$ at $56^{\circ} \mathrm{C}$. After the DTT/ $\mathrm{NH}_{4} \mathrm{HCO}_{3}$ solution was removed, gel pieces were supplemented with $150 \mu \mathrm{l} 55 \mathrm{mM}$ iodoacetamide/100 $\mathrm{mM} \mathrm{NH}_{4} \mathrm{HCO}_{3}$. Samples were incubated for $45 \mathrm{~min}$ (RT) in the dark with occasional vortexing and iodoacetamide was removed. Gel pieces were washed with $150 \mu \mathrm{l} 100 \mathrm{mM} \mathrm{NH}_{4} \mathrm{HCO}_{3}$ first and second with $150 \mu$ l acetonitrile (each 10 min incubation). Washing with $100 \mathrm{mM} \mathrm{NH}_{4} \mathrm{HCO}_{3}$ and acetonitrile was repeated two times more. Washed gel pieces were dried in the Eppendorf concentrator 5301 for 10 min and covered with sufficient trypsin digest buffer. After incubation on ice for $45 \mathrm{~min}$, excessive trypsin digest buffer was removed and $25 \mathrm{mM} \mathrm{NH}_{4} \mathrm{HCO}_{3}$, was added followed by incubation at $37^{\circ} \mathrm{C}$ over night. Samples were centrifuged at $13,000 \mathrm{rpm}$ and the liquid was transferred to a fresh $1.5 \mathrm{ml}$ Protein LoBind Tube. In the next steps peptides were eluted from gel pieces and collected in $1.5 \mathrm{ml}$ Protein LoBind Tubes. To extract the peptides from gel pieces $40 \mu \mathrm{L}$ of $20 \mathrm{mM} \mathrm{NH}_{4} \mathrm{HCO}_{3}$ was added and the samples were incubated for $10 \mathrm{~min}$. The liquid removed from the gel pieces was collected. An acetonitrile $(50 \%) / 5 \%$ formic acid mixture was added to the gel pieces followed by 20 min incubation. Again the liquid was collected and elution was performed twice more. For the pulldowns the eluates of the five upper and the five lower pieces (of one lane) were combined and for the negative controls, peptides from all 10-gel pieces were pooled. The collected liquid was dried in the Eppendorf concentrator 5301.

Purification of peptides was performed with the C18 stop and go extraction (stage) tips according to (Rappsilber et al., 2003) and (Rappsilber et al., 2007). Two small disks were punched out from an Empore ${ }^{\mathrm{TM}}$ Solid Phase Extraction (SPE) Disk C18/47mm with a 16 gauge, Metal Hub Needle (Hamilton, USA, 7748-02) and pushed into a 100-200 $\mu$ l pipette tips. Equilibration of the C18 column was performed with $100 \mu \mathrm{l}$ methanol/0.1\% formic acid, followed by $100 \mu \mathrm{l} 70 \%$ acetonitrile/0.1\% formic acid and washed twice with $100 \mu \mathrm{l} \mathrm{H}_{2} \mathrm{O} / 0.1 \%$ formic acid. Each centrifugation step was performed with the help of an adaptor in $2 \mathrm{ml}$ reaction tubes at 13,000 rpm for 2 min. 
Peptides were solved in $20 \mu \mathrm{l}$ LC/MS sample buffer $\left(\mathrm{H}_{2} \mathrm{O} / 2 \%\right.$ acetonitrile/0.1\% formic acid) and applied to the stage tips. Samples were centrifuged at $4000 \mathrm{rpm}$ for $5 \mathrm{~min}$ and the flow-trough was applied once more on the stage tip to increase the yield. Washing was performed twice with 100 $\mu \mathrm{H} \mathrm{H}_{2} \mathrm{O} / 0.1 \%$ formic acid, each time followed by centrifugation at 10,000 rpm for $2 \mathrm{~min}$. Peptides were eluted with $60 \mu \mathrm{L} \mathrm{70 \%} \mathrm{acetonitrile} \mathrm{with} 0.1 \%$ formic acid (centrifugation $5 \mathrm{~min}, 4000 \mathrm{rpm}$ ). Peptide solution was dried in the Eppendorf concentrator 5301 and peptides were solved in $20 \mu \mathrm{L}$ fresh LC/MS sample buffer.

The eluted peptide samples were analyzed by LC/MS (Hellman et al., 1995, Speicher et al., 2000). Each pull-down and LC/MS was performed in three biological replicates resulting in data from LC/MS1, LC/MS2 and LC/MS3. PRO11 or SmMOB3 pull-downs and LC/MS were performed in parallel. SCI1eGFP pull-down and LC/MS was also performed in three biological replicates (Table 8).

Table 8: Data from PRO11, SmMOB3 and SCI1 pull-down experiments coupled to LC/MS

\begin{tabular}{l|ll|ll} 
Pull-down/LC/MS & Bait & Designation & Negative control & Designation \\
\hline $\mathbf{1}$ & HAPRO11 & ER36-37 & HA & ER34-35 \\
\hline $\mathbf{2}$ & HAPRO11eGFP & ER33 & GFP & ER32 \\
$\mathbf{2}$ & HAPRO11 & ER44-45 & HA & ER46-47 \\
$\mathbf{3}$ & HAPRO11eGFP & ER49 & GFP & ER48 \\
\hline $\mathbf{3}$ & HAPRO11 & ER66-67 & HA & ER62-63 \\
\hline $\mathbf{1}$ & HAPRO11eGFP & ER65 & GFP & ER64 \\
\hline $\mathbf{1}$ & FLAGSmMOB3 & ER40-41 & FLAG & ER42-43 \\
\hline $\mathbf{2}$ & SmMOB3eGFP & ER39 & GFP & ER38 \\
\hline $\mathbf{2}$ & FLAGSmMOB3 & ER54-55 & FLAG & ER50-51 \\
\hline $\mathbf{3}$ & SmMOB3eGFP & ER53 & GFP & ER52 \\
\hline $\mathbf{3}$ & FLAGSmMOB3 & ER60-61 & FLAG & ER56-57 \\
\hline $\mathbf{1}$ & SmMOB3eGFP & ER59 & GFP & ER58 \\
\hline $\mathbf{2}$ & SCI1eGFP & ER70-71 & GFP & ER68-69 \\
\hline $\mathbf{3}$ & SCI1eGFP & ER74-75 & GFP & ER72-73 \\
\hline
\end{tabular}

The number of pull-downs and LC/MS experiments is given. For each pull-down coupled to LC/MS a corresponding negative control was prepared. Designation of LC/MS data derived from baits and the negative controls are presented. 


\subsubsection{Liquid chromatography-mass spectrometry analyses}

Liquid chromatography-mass spectrometry analysis was performed by the Mass Spectrometry Yeast group, Dr. Oliver Valerius (Institute of Microbiology and Genetics Department of Molecular Microbiology \& Genetics, Prof. Dr. Gerhard Braus, Göttingen). Proteins were identified by LC-MS with an Orbitrap Velos Pro ${ }^{\text {м }}$ Hybrid Ion Trap-Orbitrap mass spectrometer. Peptide solutions (1-5 $\mu \mathrm{l}$ ) were loaded and washed on an Acclaim ${ }^{*}$ PepMAP 100 pre-column (\#164564, $100 \mu \mathrm{m} \times 2 \mathrm{~cm}, \mathrm{C} 18,3 \mu \mathrm{m}, 100 \AA$, Thermo Fisher Scientific) with $100 \%$ loading solvent $A$ $\left(98 \% \mathrm{H}_{2} \mathrm{O}, 2 \%\right.$ acetonitrile, $0.07 \%$ trifluoracetic acid (TFA)). The flow rate was $25 \mu \mathrm{l} / \mathrm{min}$ for $6 \mathrm{~min}$. Reverse phase chromatography was applied to separate peptides on an Acclaim $*$ PepMAP RSLC column (75 $\mu \mathrm{m} \times 25 \mathrm{~cm}$ (\#164536) or $50 \mathrm{~cm}$ (\#164540), C18, $3 \mu \mathrm{m}, 100 \AA$, Thermo Fisher Scientific) using a gradient from $98 \%$ solvent $\mathrm{A}\left(\mathrm{H}_{2} \mathrm{O}, 0.1 \%\right.$ formic acid) and $2 \%$ solvent $\mathrm{B}(80 \%$ acetonitrile, $20 \% \mathrm{H}_{2} \mathrm{O}, 0.1 \%$ formic acid) to $42 \%$ solvent $B$ for $95 \mathrm{~min}$ and to $65 \%$ solvent $B$ for the following 26 min at a flow rate of $300 \mathrm{nl} / \mathrm{min}$. Peptides eluting from the chromatographic column were on-line ionized by nanoelectrospray at $2.4 \mathrm{kV}$ with the Nanospray Flex Ion Source (Thermo Fisher Scientific). Full scans of lonized peptides within a mass range of 300-1850 m/z were recorded with the Orbitrap FT analyzer at a resolution of 30,000. Collision induced dissociation (CID) fragmentation of data-dependent top-ten peptides was performed with the LTQ Velos Pro linear ion trap. For data acquisition and programming, the XCalibur 2.2 software (Thermo Fisher Scientific) was used.

\subsubsection{Y2H and drop test}

Yeast two-hybrid experiments (Fields \& Song, 1989) were performed using the pGADT7 and pGBKT7 vector system in the S. cerevisiae PJ69-4A strain (James et al., 1996).

Plasmids encoding sci1 fragments fused to the BD sequence were generated. S. macrospora cDNA was used as template for amplification of the respective sci1 fragments. The primer pairs for generation of the respective fragments containing $29 \mathrm{bp}$ overhangs to pGBKT7 are summarized in table 9. For homologous recombination, the respective fragment presented in table 9 were cotransformed with Pstl hydrolyzed pGBKT7 into the yeast strain AH109 (2.2.2).

The pro11 fragment encoding the AA region 1-110 of PRO11 needed to be cloned into the pGBKT7 background. The fragment was amplified from plasmid pBD-pro11(1-110) (401 bp) and inserted into the Pstl hydrolyzed pGBKT plasmid by homologous recombination in AH109 (2.2.2).

The AD plasmids containing the FAR genes were generated by ligation. FAR3, FAR7 and FAR 8 were amplified from PJ69-4A gDNA. FAR3 amplified with the primer pair Far3ph_f/Far3_r (662 bp) contained additional bp for hydrolysis with Ndel. Ndel hydrolyzed FAR3 PCR product and pGADT7 were ligated to generate the plasmid pADFar3. FAR7 was amplified with the primer pair Far7ph_f/ Far7flag_r. The FAR7 fragment (622 bp) was ligated into Smal hydrolyzed pGADT7. FAR8 was amplified with the primer pair Far8phf/Far8phr (1571 bp) and was also ligated into pGADT7 hydrolyzed with Smal. Plasmids pADFar7 and pADFar8 were generated by blunt end ligation (2.2.8.4). All $\mathrm{Y} 2 \mathrm{H}$ plasmids were transformed into $E$. coli and verified by sequencing. 
Table 9: Primer pairs used to amplify sci1 fragments for generation of pGBKT7 plasmids encoding sci1 fragments

\begin{tabular}{|c|c|c|c|c|c|c|}
\hline $\begin{array}{l}\text { Forward } \\
\text { primer }\end{array}$ & $\begin{array}{l}\text { Reverse } \\
\text { primer }\end{array}$ & $\begin{array}{l}\text { Amplified } \\
\text { fragment }\end{array}$ & $\begin{array}{l}\text { Fragment } \\
\text { size in bp }\end{array}$ & $\begin{array}{l}\text { Hydrolyzed } \\
\text { backbone }\end{array}$ & $\begin{array}{l}\text { Enzyme for } \\
\text { hydrolysis }\end{array}$ & $\begin{array}{l}\text { Resulting } \\
\text { plasmid }\end{array}$ \\
\hline sci1_Y1f & sci1_Y1r & $\operatorname{sci}_{1-62}$ & 187 & pGBKT & Pstl & pBDsci $1_{1-62}$ \\
\hline sci1_Y1f & sci1_Y2r & $\operatorname{sci1}_{1-120}$ & 361 & pGBKT & Pstl & pBDsci1 $1_{1-120}$ \\
\hline sci1_Y5f & sci1_Y5r & sci1 $100-155$ & 165 & pGBKT & Pstl & pBDsci1 $1_{100-155}$ \\
\hline sci1_Y2f & sci1_Y3r & $\operatorname{sci}_{63-207}$ & 437 & pGBKT & Pstl & pBDsci1 $1_{63-207}$ \\
\hline sci1_Y3f & sci1_Y3r & sci1 $_{121-207}$ & 263 & pGBKT & Pstl & pBDsci1 $1_{121-207}$ \\
\hline sci1_Y3f & sci1_Y4r & sci1 $_{121-298}$ & 536 & pGBKT & Pstl & pBDsci1 $1_{121-298}$ \\
\hline sci1_Y4f & sci1_Y4r & sci1 $_{208-298}$ & 275 & pGBKT & Pstl & $\mathrm{pBDsci}_{208-298}$ \\
\hline
\end{tabular}

The numbers given in the amplicons represent the amino acids of scil encoded in the corresponding generated fragments

The $A D$ and $B D$ plasmids were cotransformed into PJ69-4A generating the strains used for the $\mathrm{Y} 2 \mathrm{H}$ drop tests. The yeast strains generated, the growth controls and the $\mathrm{Y} 2 \mathrm{H}$-drop testes are presented in figure 16, figure 19 and figure $S 9$, respectively.

The pGADT7 encodes the activation domain (AD) sequence and pGBKT7 encodes the binding domain (BD) sequence of the transcriptional activator GAL4. When the fusion proteins interact, Gal4 activates transcription of the reporter genes HIS3 and $A D E 2$, which allows synthesis of adenine and histidine and growth of PJ69-4A on SD medium without adenine and histidine (SD-Ade, -Trp). As a positive control and test of the system competency, yeast strains were transformed with plasmids encoding the AD fused to the Gal4-BD interacting protein RanBPM (Tucker et al., 2009) and $\mathrm{BD}$ fused to $\mathrm{SCl1}, \mathrm{PRO} 11$ or PRO45 protein fragments. For the negative controls, yeast strains containing empty plasmids pGADT7 or pGBKT7 and corresponding sci1, pro11 or pro 45 fragments fused to the BD or AD sequence (respectively) were used (James et al., 1996). A drop test was performed and yeast growth was observed on synthetic defined (SD) medium without leucine (Leu), tryptophan (Trp), adenine (Ade) and histidine (His) (-Leu/Trp/ Ade/ His, -Leu/Trp/ Ade, -Leu/ Trp/ His and -Leu/Trp. To do this, yeast strains were grown in liquid SD -Leu/-Trp to log phase and were diluted to an $\mathrm{OD}_{600}$ of 0.1 . The 0.1 dilution and a 1:10 dilution series (of each $20 \mu \mathrm{L}$ ) were spotted onto selective SD medium. Yeasts were incubated for 3 days at $30{ }^{\circ} \mathrm{C}$ and pictures were taken with the BioDocAnalyzer (Biometra, Germany). 


\subsubsection{Analytical methods}

\subsubsection{Light and fluorescence microscopy}

For light microscopy the Axiolmager M1 microscope (Zeiss, Jena, Germany) was used. The images were taken with the Photometrics CooISNAP HQ2 camera (Roper Scientific, Photometrics, Tucson, Arizona) and were edited with MetaMorph (version 6.3.1; Universal Imaging).

\section{Investigation of sexual structures}

For the investigation of sexual structures, strains were grown for 2-8 days and documented with differential interference-contrast (DIC) microscopy. All S. macrospora strains were grown on BMM covered glass slides and $2 \mathrm{~mL}$ liquid BMM was put on top.

\section{Investigation of hyphal fusion events}

For investigation of hyphal fusion events with DIC microscopy, strains were grown on cellophanecovered minimal medium containing soluble starch (MMS) plates for 3 days at $27^{\circ} \mathrm{C}$ (Rech et al., 2007).

\section{Counting of protoperithecia}

Wild type, $\Delta$ sci $_{\text {hyg }}$ and $\Delta$ sci1 $1_{\text {hyg }}: 5$ 'scilegfp strains were grown on BMM covered slides and DIC microscopy was used to count protoperithecia with a size of 40-200 $\mu \mathrm{m}$. The fast growing wt was

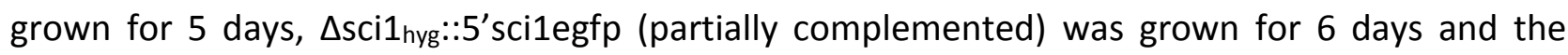
$\Delta$ sci $1_{\text {hyg }}$ strain, which shows a very slow growth phenotype was grown for 7 days. For the three strains, similar regions for protoperithecia counting were defined. Protoperithecia were counted 5

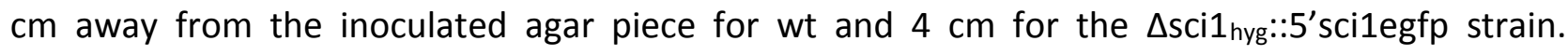
Protoperithecia in the $\Delta$ sci $1_{\text {hyg }}$ strain were counted $3 \mathrm{~cm}$ away from the inoculated agar piece. One $\mathrm{cm}^{2}$ of mycelium of each strain was used to count protoperithecia in five independent experiments $(n=5)$.

\section{Documentation of $S$. macrospora grown on agar plates}

Pictures for S. macrospora strains or mycelium grown on agar plates were taken with the Digital Microscope VHX-500F (Keyence, Germany).

\section{Fluorescence microscopy}

CFW staining (Sigma-Aldrich, 18909) diluted 1:1 with 10\% KOH was applied onto mycelium grown on BMM-covered slides to visualize cell walls and septa.

The 10 mM CMAC stock solution (CMAC solved in DMSO) was diluted 1:400 in $\mathrm{H}_{2} \mathrm{O}$ and 50-100 $\mu \mathrm{L}$ were applied directly on S. macrospora mycelium. The samples were incubated for $30 \mathrm{~min}$ at $37^{\circ} \mathrm{C}$. For staining of lipid rich membranes $50-100 \mu \mathrm{L} F 4-64\left(1 \mu \mathrm{g} / \mathrm{mL} \mathrm{H}_{2} \mathrm{O}\right)$ was directly applied onto S. macrospora mycelium and fluorescence microscopy was performed after 10-20 min incubation at RT.

For the detection of the GFP, DsRED/TdTomato/FM4-64, DAPI/CMAC signals and calcofluor white (CFW) fluorescence chroma filter set 49002, 49005, 49000 and 89002 (exciter ET470/40x, ET545/30x, ET350/50x, ET430/24x/ET500/20x, emitter ET525/50m, ET620/60m, ET460/50m, 
ET470/24m/ET535/30m and beamsplitter T495Ipxr, T570lp, T400Ip, 69008bs) were used, respectively.

\subsubsection{DNA and protein sequence analysis}

The Clone Manager 7, 7.04 software (Sci Ed Central, 1994-2002 Scientific \& Educational Software) was used to generate plasmid sequences. For analysis of sequenced plasmids, sequence alignments were performed with the Nucleotide BLAST database (Altschul et al., 1990) (http://blast.ncbi.nlm.nih.gov/Blast.cgi). Also, DNA and protein sequences were obtained from the BLAST database. ExPASy GPSDB Gene/Protein Synonyms finder was used to identify orthologs of small coiled-coil proteins associated with the STRIPAK complex (Pillet et al., 2005). ExPASY Compute pl/MW tool (http://web.expasy.org/compute_pi/) was used to calculate the isoelectric point and the molecular weight of proteins. The program SMART 7.0 (Letunic et al., 2015, Schultz et al., 1998) (http://smart.embl-heidelberg.de/) was used to predict protein domains. The MPI Bioinformatics Toolkit COILS/PCOILS (Alva et al., 2016) (https://toolkit.tuebingen.mpg.de) and the ExPASy COILS software (Lupas et al., 1991) (http://embnet.vital-it.ch/software/COILS_form.html) were used to identify coiled-coil domains in proteins.

\subsubsection{Phylogenetic analysis}

Multiple sequence alignment of protein sequences was performed with Mafft version 7.182 (Katoh \& Standley, 2013). Phylogentic analysis was performed with the program PHYLIP, version 3.6 (http://evolution.genetics.washington.edu/phylip.html) (Felsenstein, 2005). The program PROTDIST was used to calculate the distance matrices, which were analysed with the NEIGHBOR program to generate phylogenetic trees. The bootstrap analysis was performed with 1000 iterations to rebuild and test the trees for statistical significance. Phylogenetic trees were generated with the program CONSENSE and displayed with the program TreeView 1.6.6. (Page, 1996). Adobe Illustrator CS2 12.0.0 was used to represent the phylogenetic trees.

\subsubsection{LC-MS data analysis: MaxQuant/Perseus and Proteome Discoverer ${ }^{\mathrm{TM}}$}

\section{Analysis of LC/MS data with MaxQuant/Perseus}

Proteins were identified with the MaxQuant 1.4.1.2. software (Cox \& Mann, 2008) using the Andromeda algorithm. S. macrospora specific protein database (Nowrousian et al., 2010) was used for database search. Default parameters were used and the digestion mode was trypsin/P. Maximum missed cleavage sites were 3 , carbamidomethylation of cysteins was considered as fixed modification. The mass tolerance was $4.5 \mathrm{ppm}$ for precursor ions and $0.5 \mathrm{Da}$ for fragment ions. The decoy mode was revert with a false discovery rate of 0.01 . Subsequent data processing and statistical analysis were performed with the Perseus 1.5.1.0 software (Cox \& Mann, 2012, Tyanova et al., 2016). 
Table 10: Example of a workflow for LC/MS data analysis with MaxQuant/Perseus

\begin{tabular}{|c|c|c|c|c|c|}
\hline Step & Command & Description & Step & Command & Description \\
\hline \multirow[t]{3}{*}{1} & $\begin{array}{l}\text { Load raw Data files into } \\
\text { MaxQuant } \\
\text { Set Experiment }\end{array}$ & $\begin{array}{l}\text { Data naming for } \\
\text { example: } \\
\text { 1GFP, } \\
\text { 1HAPRO11eGFP } \\
2 \text { GFP, } \\
2 \text { HAPRO11eGFP } \\
3 G F P \text {, } \\
3 \text { HAPR011eGFP }\end{array}$ & 6 & $\begin{array}{l}\text { Processing } \\
\text { Filter rows } \\
\text { Filter rows based } \\
\text { on valid values }\end{array}$ & $\begin{array}{l}\text { Number of values } 2 \\
\text { In at least one group: } \\
\text { Group1 } \\
\text { Values should be: Valid }\end{array}$ \\
\hline & $\begin{array}{l}\text { Global Parameters: } \\
\text { Insert Fasta file } \\
\text { Requantify }\end{array}$ & $\begin{array}{l}\text { Fasta file: } \\
\text { Peptide library }\end{array}$ & 7 & $\begin{array}{l}\text { Processing } \\
\text { Imputation } \\
\text { Replace missing } \\
\text { values from } \\
\text { normal } \\
\text { distribution }\end{array}$ & $\begin{array}{l}\text { Replace NaN, } \\
\text { Width: } 0.3 \\
\text { Down shift: } 1.8 \\
\text { Mode: Separately for } \\
\text { each columns }\end{array}$ \\
\hline & $\begin{array}{l}\text { Group specific parameters: } \\
\text { Lable-free quantification }\end{array}$ & $\begin{array}{l}\text { LFQ yes, default } \\
\text { settings } \\
\text { Calculation } \\
\text { done by } \\
\text { MaxQuant }\end{array}$ & 8 & $\begin{array}{l}\text { Analysis } \\
\text { Clustering /PCA } \\
\text { Hierarchical } \\
\text { clustering } \\
\text { Uncheck Column } \\
\text { tree }\end{array}$ & $\begin{array}{l}\text { heat map } \\
\text { Adjust Colors } \\
\text { Adjust data } \\
\text { representation } \\
\text { row/column height, } \\
\text { width, Protein IDs }\end{array}$ \\
\hline 2 & $\begin{array}{l}\text { Generic upload Perseus } \\
\text { Expression: LFQ intensity } \\
\text { Numerical: PEP (Posterior } \\
\text { error probability), Intensity, } \\
\text { Razor + unique peptides, } \\
\text { Sequence coverage, } \\
\text { Peptides, unique Peptides } \\
\text { Categorial: Only identified } \\
\text { by site, Reverse, } \\
\text { Contaminant, Text: Protein } \\
\text { IDs, Majority protein IDs }\end{array}$ & $\begin{array}{l}\text { proteinGroups.t } \\
\text { xt }\end{array}$ & 9 & $\begin{array}{l}\text { Processing } \\
\text { Tests } \\
\text { Two-samples } \\
\text { tests }\end{array}$ & $\begin{array}{l}\text { Grouping: Group1, for } \\
\text { example } \\
\text { First Group: GFP } \\
\text { Second Group: } \\
\text { HAPRO11eGFP } \\
\text { Test: t-test } \\
\text { S0: } 2 \\
\text { Side: both } \\
\text { FDR:0.01 or } 0.1 \text { (False } \\
\text { discovery rate) }\end{array}$ \\
\hline 3 & $\begin{array}{l}\text { Processing } \\
\text { Filter rows based on } \\
\text { categorical column }\end{array}$ & $\begin{array}{l}\text { Only identified } \\
\text { by site } \\
\text { Reverse } \\
\text { Contaminant }\end{array}$ & 10 & $\begin{array}{l}\text { Analysis } \\
\text { Basic } \\
\text { visualization } \\
\text { scatter plot }\end{array}$ & $\begin{array}{l}x \text {-axis: t-test Difference } \\
y \text {-axis: -Log t-test } p \\
\text { value } \\
\text { Vulcano plot }\end{array}$ \\
\hline 4 & $\begin{array}{l}\text { Processing } \\
\text { Basic } \\
\text { Transform }\end{array}$ & $\log 2(x)$ & & & $\begin{array}{l}\text { Selected labels } \\
\text { t-test Significant } \\
\text { Vulcano plot: }\end{array}$ \\
\hline 5 & $\begin{array}{l}\text { Processing } \\
\text { Annotation rows } \\
\text { Categorical annotation } \\
\text { rows }\end{array}$ & $\begin{array}{l}\text { Create Group1 } \\
\text { Define which } \\
\text { samples belong } \\
\text { to the negative } \\
\text { control and } \\
\text { which are the } \\
\text { samples of } \\
\text { interest (for } \\
\text { example GFP, } \\
\text { HAPRO11eGFP) }\end{array}$ & & & $\begin{array}{l}\text { Categories, click on t-test } \\
\text { Significant } \\
\text { Selected labels } \\
\text { Protein IDs }\end{array}$ \\
\hline
\end{tabular}


For analysis with Perseus, label free quantification based on signal intensities was applied. Pulldown samples were analyzed from HAPRO11 (MS1-3) or HAPRO11eGFP (MS1-3), or FLAGSmMOB3 (MS1-3) or SmMOB3eGFP (MS1-3) or SCI1eGFP (MS1-3) (Table 8). To identify specifically enriched protein criteria were as follows, enriched in at least two LC/MS experiments compared to the corresponding negative controls. Proteins enriched in all three pull-downs were considered as highly confident.

The work flow used to analyze the LC/MS data with MaxQuant/Perseus in this work is presented in table 10. However, the workflow for analysis of LC/MS data is dependent on the needs of analysis and the obtained LC/MS data should be elaborated individually.

For the heat-map representation, intensity signals were set as follows, intensity very high (dark blue, 26), intensity high (blue, 24), intensity medium (light blue, 20-22), and intensity low (white, 18). A t-test was performed using Perseus and the results were represented by a volcano plot. Criteria for specifically enriched proteins were t-test difference $<-3$ and $p$-value $<0.1$.

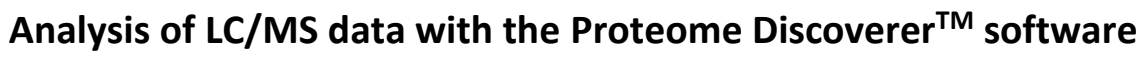

Proteins were also identified with the Proteome Discoverer ${ }^{\mathrm{TM}} 1.4$ software (Thermo Scientific, version 1.4.0288) using SequestHT (Thermo Scientific) and Mascot search algorithms. The database searches were performed against a S. macrospora specific protein database (Nowrousian et al., 2010) extended by the most common contaminants. For the maximum of misscleavage sites two residues were expected and the digestion mode was trypsin. Fixed modification was carbamidomethylation of cysteins. The mass tolerance was $10 \mathrm{ppm}$ for precursor ions and $0.6 \mathrm{Da}$ for fragment ions. The decoy mode was revert.

With the Proteome Discoverer ${ }^{T M}$ LC/MS Data from each biological replicate of PRO11 (HAPRO11 and HAPRO11eGFP MS1, HAPRO11 and HAPRO11eGFP MS2 or HAPRO11 and HAPRO11eGFP MS3) or SmMOB3 (FLAGSmMOB3 and SmMOB3eGFP MS1, FLAGSmMOB3 and SmMOB3eGFP MS2 or FLAGSmMOB3 and SmMOB3eGFP MS3) or and corresponding negative controls (Table 8) were pooled and analyzed using spectral counts. Also, for SCI1eGFP each biological replicate (MS1-3) and the negative control eGFP were analyzed using spectral counts. The settings in the Proteome Discoverer $^{\mathrm{TM}}$ were as follows, high peptide confidence and minimum 2 peptides with a false discovery rate less than 0.01 . Criteria for specifically enriched proteins were, no peptides enriched in the negative control and at least 7 peptide counts in two of three LC/MS experiments. Comparing putative interaction partners found in PRO11 and simultaneously in SmMOB3 pull downs criteria were less stringent, no peptides enriched in the negative control and at least 5 peptide counts in two of three LC/MS experiments. The LC/MS Excel lists with the sum of spectral counts are supplemented on a digital video disk.

\subsubsection{Measures of safety}

Genetic engineering experiments of security level 1 have been conducted according to the guide lines of the genetic engineering law (GenTG) stated on 16.12.1993 (recently altered by Art. $12 \mathrm{G}$ v. 29.7.2009 ( 2542). 
Results

\section{Results}

\subsection{Pull-down experiments and LC/MS with the STRIPAK proteins PRO11 and SmMOB3}

The STRIPAK complex of S. macrospora consists of the ortholog of striatin PRO11, the kinase activator SmMOB3, the STRIP1/2 ortholog PRO22, the SLMAP ortholog PRO45, the phosphatase scaffolding subunit SmPP2AA and the catalytic phosphatase subunit SmPP2Ac1 (Beier et al., 2016, Bernhards \& Pöggeler, 2011, Bloemendal et al., 2012, Bloemendal et al., 2010, Nordzieke et al., 2015). PRO11 is thought to act as scaffolding protein for the association of the STRIPAK components and interacts with SmMOB3 (Bloemendal et al., 2012). Thus, by means of liquid chromatography-mass spectrometry (LC/MS) proteins that associate with PRO11 and SmMOB3 were identified in this study.

\subsubsection{LC/MS data analysis with MaxQuant/Perseus identified a putative interaction partner of PRO11 with high confidence}

To identify putative interaction partners of HAPRO11, total protein content from $\Delta$ pro11::hapro11 (Bloemendal et al., 2012) and wt::ha (negative control) mycelium was enriched by HA-Trap, resolved in SDS-PAGE and digested with trypsin. The eluted peptide samples were analyzed by LC/MS (Hellman et al., 1995, Speicher et al., 2000). The data were analyzed in a semiquantitative method using MaxQuant/Perseus (Cox \& Mann, 2008, Cox \& Mann, 2012, Tyanova et al., 2016) and label-free quantification (LFQ) based on signal intensities (section 1.6.11.4). Proteins enriched in at least two LC/MS experiments but not in the corresponding negative control were considered as putative interaction partners. The overall number of proteins identified in the respective pulldowns is given in table 11. Candidates enriched in all three LC/MS experiments were considered as high confidence interaction partners. MaxQuant/Perseus data analysis was visualized in a heat map and a volcano plot (Figure 8).

In the heat map high signal intensities are indicated by dark colors and low signal intensities are indicated by light colors. For simplicity only the signal intensities for the first 20 detected proteins are presented in figure 8A. Peptides of the HAPRO11 bait protein were identified with high signal intensity in all three LC/MS replicates colored in dark blue. Furthermore, peptides of the known PRO11 (SMAC_08794) interaction partner PRO22 (SMAC_02580) and a protein with the locus tag 


\section{Results}

SMAC_05559 were copurified. The new protein was named STRIPAK omplex interactor 1 (SCI1). SCI1 was highly enriched by HAPRO11 in the first and second pull down and moderately enriched in the third pull-down experiment. PRO22 was also highly enriched by HAPRO11 in the first and second pull down but only slightly enriched in the third pull-down. PRO11, PRO22 and SCI1, were not enriched in the negative control (Figure $8 \mathrm{~A}$ ).
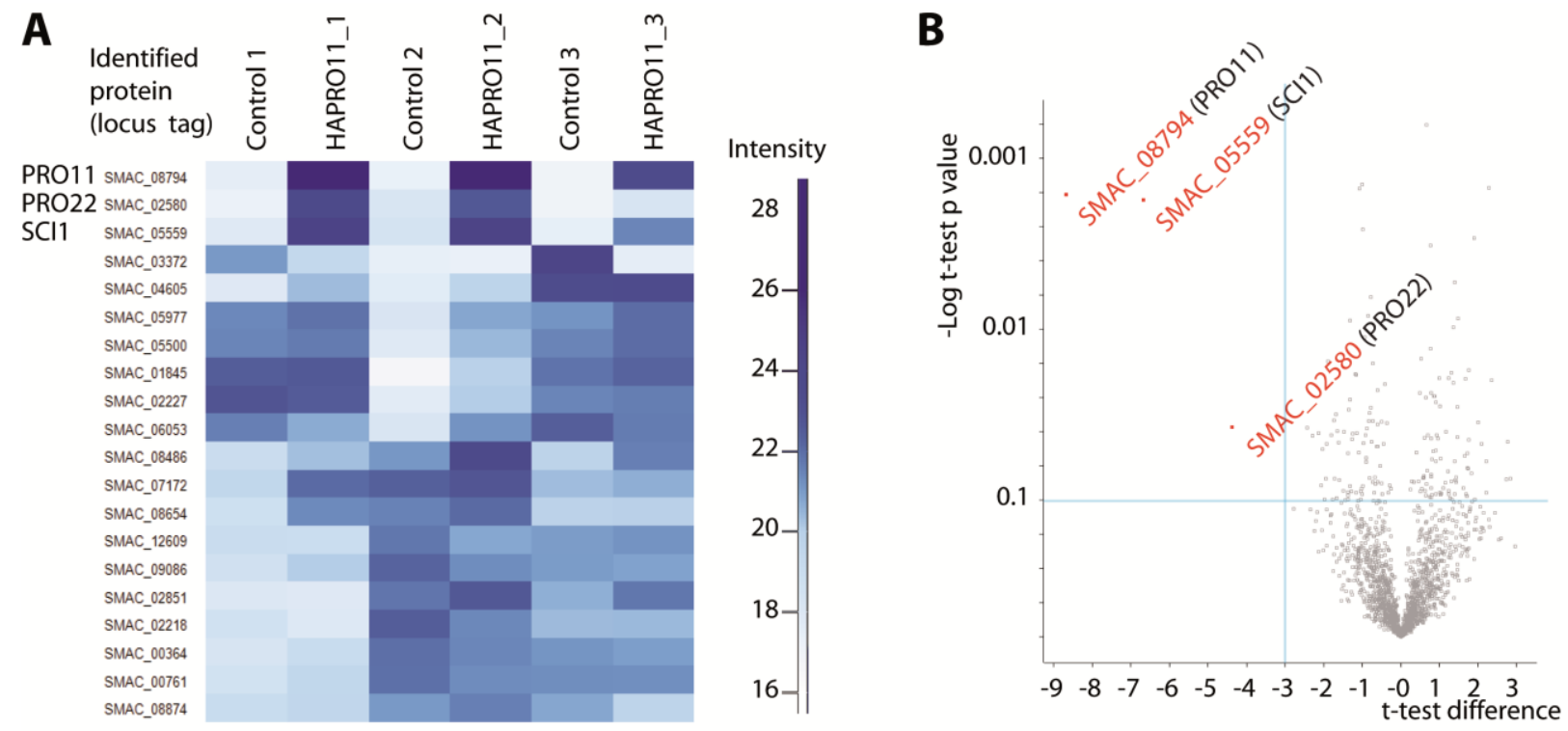

Figure 8: LC/MS data from HA-Trap with HAPRO11 as bait represented in a heat map and a volcano plot In the heat map and the volcano plot the results of the HA-Trap and LC/MS are represented. A: The heat map shows the results of the HAPRO11-LC/MS1, 2 and 3 with the corresponding HA negative controls 1, 2 and 3. Signal intensities correlate with the abundance of the peptides detected. White represents low signal intensities and dark blue indicates high signal intensities. In total, 1229, 1103 and 1529 proteins were identified with MaxQuant/Perseus but for simplicity only the results for the first 20 identified proteins are represented in the heat map. Signal intensities for the bait protein with the locus tag SMAC_08794 (PRO11) were high in all LC/MS experiments. PRO22 (SMAC_02580) was highly enriched in LC/MS1 and 2 and SCl1 (SMAC_05559) was enriched in all three LC/MS experiments. B: The volcano plot represents the results of a statistical 2 sample t-test. Putative PRO11 interaction partners have a $p$-value $<0.1$ and a t-test difference value of $<-3$.

A statistical 2-sample t-test was performed using signal intensities, and the results are presented as volcano plot to discriminate significant candidates identified in the HAPRO11 pull downs from unspecific background. Identified proteins with a t-test difference $>0$ represent the identified proteins detected in the negative control and $<0$ represent proteins enriched with HAPRO11 (Figure 8B). Candidates classified as unspecific background have a t-test difference $>-3$. Candidates having a $p$ value $<0.1$ and a t-test difference $<-3$ were classified as significant and thus putative 
PRO11 interaction partners. Here, PRO11 groups together with its known interaction partner PRO22 and with the novel protein SCl1 (Figure 8B).

Also, total protein content was isolated from pro11::hapro11egfp (Herzog and Pöggeler unpublished) and from wt::egfp (Voigt \& Pöggeler, 2013). GFP-Trap and LC/MS was performed and the data was evaluated to the same criteria as described before. Neither PRO22 nor SCI1 could be enriched with HAPRO11eGFP expressed in the pro11 mutant. However, SmMOB3, a known interaction partner of PRO11, was identified as high confidence interaction partner of the HAPRO11eGFP fusion protein (Figure S 1 A/B). The results also apply vice versa, SmMOB3 was not enriched in the HAPRO11 pull-down (Figure S 1 A/B, Figure 8).

Also the total protein contents were isolated from the fus::flagSmmob3 and the $\Delta$ Smmob3_gpdMOB3:GFP strain and the corresponding negative controls wt::flag/wt::egfp, followed by FLAGTrap or GFP-Trap, respectively coupled to LC/MS.

However, only in the SmMOB3eGFP pull-downs PRO11 was identified as high confidence interaction partner and no further interaction partners matching the criteria were identified in FLAGSmMOB3 and SmMOB3eGFP pull-downs (Figure S $1 \mathrm{C} / \mathrm{D}$ and E/F). The overall number of different proteins identified with MaxQuant/Perseus in the respective pull-downs is given in table 11. The complete heat maps for PRO11 and SmMOB3 pull-downs are presented in figure S 2 and the position of the protein used for the respective pull-down is indicated by an arrow.

Table 11: Sum of proteins identified with MaxQuant/Perseus from PRO11 and SmMOB3 pull- downs

\begin{tabular}{c|c|c|c|c|}
\hline Pull-down & LC/MS1 & LC/MS2 & LC/MS3 & LC/MS1, 2 and 3 \\
\hline HAPRO11 & 1229 & 1103 & 1529 & 1822 \\
\hline HAPRO11eGFP & 714 & 744 & 1060 & 1293 \\
\hline FALGSmMOB3 & 1461 & 1644 & 1563 & 2018 \\
\hline SmMOB3eGFP & 695 & 875 & 986 & 1180 \\
\hline
\end{tabular}

The overall number of different proteins (specific and unspecific) identified from the indicated pull-downs with the MaxQuant/Perseus software is presented. The number of all different proteins identified altogether in LC/MS1, 2 and 3 is also given. 


\subsubsection{LC/MS Data analysis with the Proteome Discoverer ${ }^{\mathrm{TM}}$ identified several putative interaction partners of PRO11 and SmMOB3}

The Proteome Discoverer ${ }^{\mathrm{TM}}$ (Thermo Scientific) was used to evaluate the data combined from HAPRO11 (LC/MS1-3) and HAPRO11eGFP (LC/MS1-3) (PRO11 pull-down) or FLAGSmMOB3 (LC/MS1-3) and SmMOB3eGFP (LC/MS1-3) (SmMOB3 pull-down) using the sum of spectral counts. The overall number of proteins identified in the respective pull-downs is given in table 12.

Table 12: Number of proteins identified with the Proteome Discoverer ${ }^{\mathrm{TM}}$ from PRO11 and SmMOB3 pulldowns

Pull-down

\begin{tabular}{c}
\hline HAPRO11 \\
HAPRO11eGFP \\
FALGSMMOB3 \\
\hline
\end{tabular}

SmMOB3eGFP
LC/MS1

901

545

943

425
LC/MS2

684

463

991

597
LC/MS3

998

717

1038

586
LC/MS1-3

1205

820

1302

748

The overall number of different proteins (specific and unspecific) identified from the indicated pull-downs with the Proteome Discoverer ${ }^{\mathrm{TM}}$ software is presented.

The criteria for analysis with the Proteome Discoverer ${ }^{\mathrm{TM}}$ were: high peptide confidence and a minimum of two peptides with a false discovery rate less than 0.01 . For potential interaction partners the criteria were: no peptides enriched in the negative control and at least 7 peptide counts. Tagged PRO11 and SmMOB3 were enriched in the respective pull-down/LC/MS experiments in large numbers of spectral counts (Table S 1, Table S 2).

In two replicates of PRO11 pull-downs, SmMOB3 (SMAC_00877) and PRO22 (SMAC_02580) were identified with minimal 7 spectral counts. Remarkably, spectral counts for SmMOB3 were high when spectral counts for PRO22 were low and vice versa (Table S 1). The protein SCI1 with the locus tag SMAC_05559 was identified in all three biological replicates of the PRO11 pull-downs in large numbers of spectral counts (Table S 1). Further, 25 possible interaction partners were identified in PRO11 pull-downs, among them 4 proteins involved in transport: a kinesin group protein (SMAC_04212), an actin-2 protein (SMAC_07827), a vacuolar sorting protein (SMAC_04470) and a Sar1-like protein (SMAC_04918). Furthermore, three kinases, two STKs (SMAC_04710 and SMAC_05353) and one STE20-like kinase (SMAC_07811), a nucleoporin (SMAC_02409) and a small GTPase of the Ras family (SMAC_07014) were identified (Table S 1). 
In SmMOB3 pull-downs PRO11 was identified in high numbers of spectral counts but no further STRIPAK member that matched the criteria. On the other hand, four unknown protein were identified. Two of them, a putative myosin-5 protein (SMAC_05008) and the AP-2 complex subunit alpha (SMAC_09495) are related to transport (Table S 2).

To get an idea which peptides are found in all PRO11 and SmMOB3 pull-downs the criteria were set as follows: high peptide confidence, minimal 2 peptides per protein, not enriched in the negative control and at least 5 peptide counts in at least two biological PRO11 and SmMOB3 replicates, respectively. PRO11, PRO22 and SCI1 were detected in almost all experiments but also the putative myosin-5 protein (SMAC_05008), a nucleoporin (SMAC_02409) and the serine/threonine-protein phosphatase 1 (PP1) were detected (Table S 3).

\subsection{Small coiled-coil proteins that associate with the STRIPAK complex in fungi and animals are conserved through their domain structure}

The protein SCI1 (SMAC_05559) was enriched in HA-Trap experiments using HAPRO11 as bait combined with LC/MS. The ORF SMAC_05559 encodes a protein of 298 amino acids (AA) with a predicted molecular weight (Mw) of $33 \mathrm{kDa}$ and an isoelectric point (pl) of 5.07. Only coiled-coil (CC) regions were identified with the SMART database (Letunic et al., 2015, Schultz et al., 1998) and in total four CC domains were predicted for the SCI1 protein with the MPI Bioinformatics Toolkit COILS/PCOILS (Alva et al., 2016) and the ExPASy COILS (Lupas et al., 1991) program.

The CC domains in SCI1 were predicted in the following AA positions: 22-55, 68-113, 138-184 and 235-267. In mammals and yeasts, small proteins associating with the STRIPAK complex contain one or more CC domains (Figure 9). In S. cerevisiae, Far3 (204 AA) and Far7 (221 AA) represent two CC domain containing protein associated with the Far complex. Far3 was predicted to have two CC domains and Far7 only one (Lai et al., 2011). In S. pombe, Csc4 (166 AA) has only one CC region and was found in the SIN-inhibitory PP2A (SIP) complex (Singh et al., 2011).

The STRIPAK complex in $H$. sapiens can associate with SIKE (207 AA) or FGFR1OP2 (253 AA), which are closely related (Goudreault et al., 2009). For SIKE, two CC domains were predicted but a third CC region between AA 2 and 46 might also exist (Huang et al., 2005a). Whereas, for FGFR1OP2 four

CC were predicted (Lin et al., 2010). In D. melanogaster, FGOP2 (315 AA) associates with the 
dSTRIPAK but is not extensively studied in fruit flies (Ribeiro et al., 2010). For FGOP2 three CC domains were predicted with COILS/PCOILS and COILS (Figure 9).

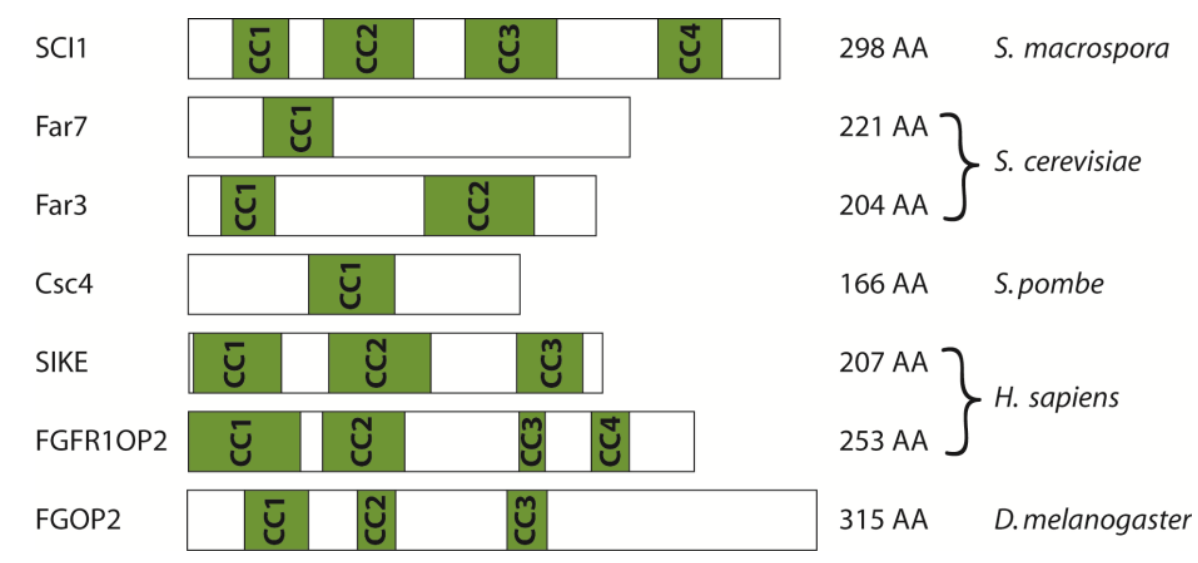

Figure 9: Domain structure of small coiled-coil proteins associated with the STRIPAK in fungi and animals Schematic representation of small CC proteins associating with the orthologous STRIPAK complexes of S. macrospora, S. cerevisiae, S. pombe, H. sapiens and D. melanogaster and their predicted domain structure. All proteins contain one or more cc regions distributed over the protein. Numbers represent the length of the corresponding protein in amino acids (AA). CC: Coiled-coil domain (green). SCI1: STRIPAK complex interactor 1 (XP_003349276), Far3 (AAB39035)/7(P43592): Factor arrest 3/7, Csc4 (O14043): Component of SIP complex, SIKE (NP_079349): Suppressor of IKKE, FGFR1OP2 (NP_001165358)/FGOP2 (NP_609084): Fibroblast growth factor receptor 1 oncogene partner 2.

\subsection{Small CC proteins associated with the STRIPAK complex are conserved within phyla belonging to distant clades}

Small proteins containing CC domains associate with the STRIPAK in different organisms (Hwang \& Pallas, 2014). However, it is not possible to determine orthologs by BLAST searches due to the low conservation of the AA sequences between animals, yeasts and filamentous fungi. Therefore, SIKE and FGFR1OP2 orthologs in animals were identified with the GPSDB Gene/Protein Synonyms finder (Pillet et al., 2005) and BLAST (Altschul et al., 1990) using human SIKE and FGFR1OP2. In filamentous fungi, BLAST search with S. macrospora SCI1 identified putative fungal SCl orthologs. Full length amino-acid sequences of SIKE/FGFR1OP2, Far3/7, Csc4 and SCl proteins were aligned with MAFFT (Katoh \& Standley, 2013) and neighbor joining was performed with the program NEIGHBOR of the program package PHYLIP (Felsenstein, 2005). Despite being barely conserved by AA sequence, small STRIPAK-associated proteins containing CC domains are conserved within phyla belonging to distant clades presented in the unrooted neighbor-joining tree (Figure 10). 


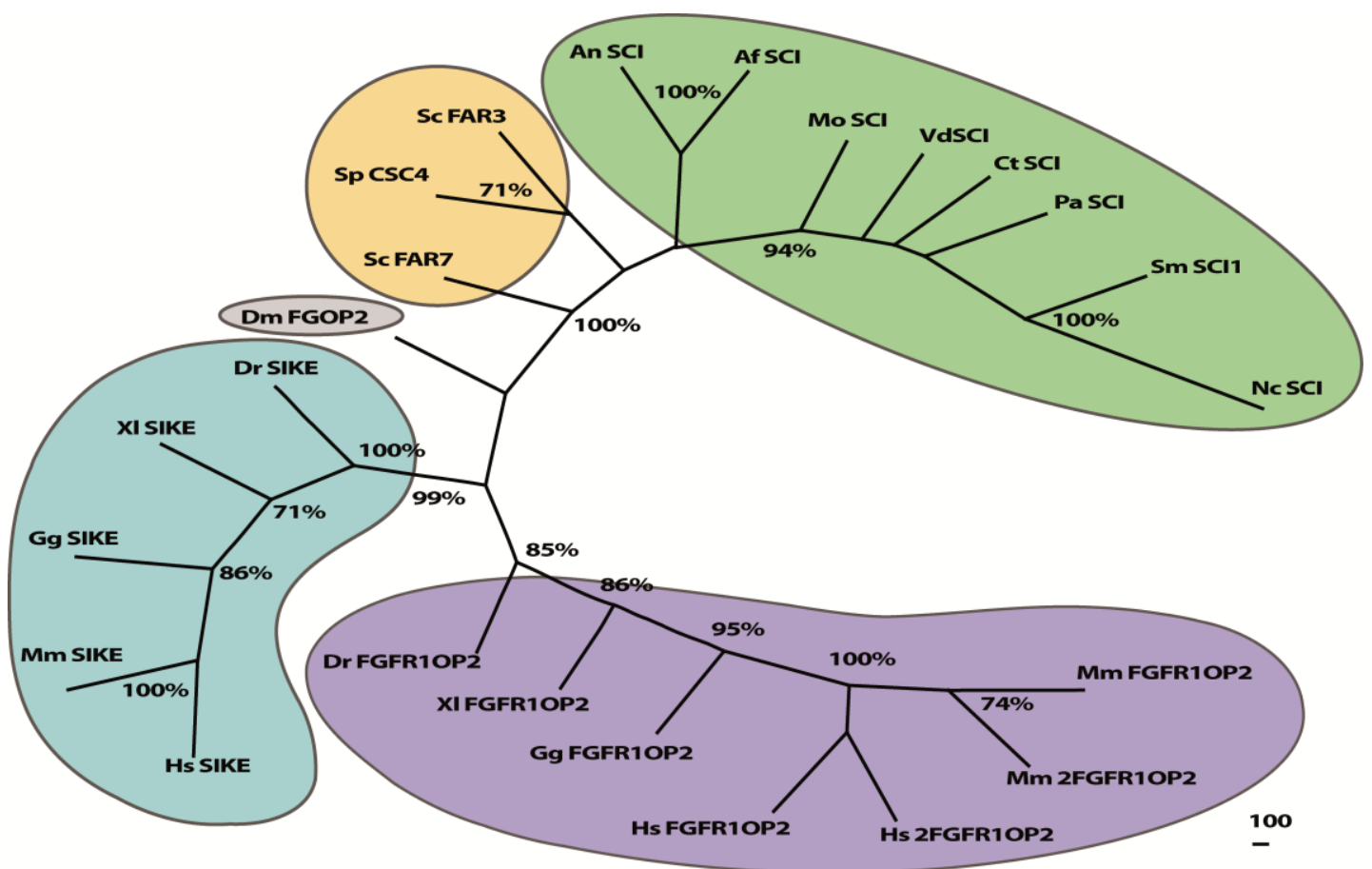

Figure 10: Phylogenetic tree of small CC proteins associated with the STRIPAK

Phylogenetic tree of STRIPAK-associated proteins containing CC domains generated with the neighborjoining method and shown in a distance matrix. Orthologs were identified with BLAST search using amino acid sequences of human SIKE and FGFR1OP2 and S. macrospora SCI1. The multiple sequence alignment was performed with MAFFT version 7.182 (Katoh \& Standley, 2013) using the amino acid sequences: Sm SCl1, S. macrospora (XP_003349276.1); Nc SCl, N. crassa (XP_960970.1); Ct SCl, Chaetomium thermophilum (XP_006696634.1); Vd SCl, Verticillium dahliae (EGY17808.1); $\mathrm{Pa} \mathrm{SCl}$, Podospora anserina

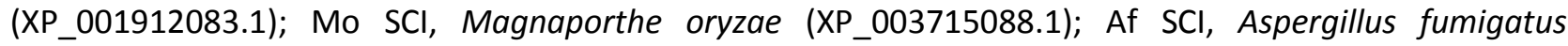
(XP_752657.2); An SCl, A. nidulans (XP_658614.1); Sc, S. cerevisiae Far3 (AAB39035) and Far7 (P43592); Sc Csc4, S. pombe Csc4 (014043); Dm FGOP2, D. melanogaster FGOP2 (NP_609084); Hs SIKE, H. sapiens SIKE (NP_079349.2); Mm SIKE, M. musculus SIKE (NP_079955.1); Gg SIKE, Gallus gallus domesticus SIKE (NP_001006324.2); XI SIKE, Xenopus laevis SIKE (NP_001085744.1); Dr SIKE, Danio rerio SIKE (XP_696578.2); Hs 1FGFR1OP2, $H$. sapiens isoform 1 FGFR1OP2 (NP_056448.1); Hs 2FGFR1OP2, H. sapiens isoform 2 FGFR1OP2 (NP_001165358.1); Mm 1FGFR1OP2, M. musculus isoform 1 FGFR1OP2 (NP_080494.1); Mm_2FGFR1OP2, M. musculus isoform 2 FGFR1OP2 (XP_006536173.1); Gg FGFR1OP2, G. domesticus FGFR1OP2 (NP_001007856.1); XI FGFR1OP2, X. laevis FGFR1OP2 (NP_001079401.1); Dr FGFR1OP2, D. rerio FGFR1OP2 (NP_956249.1). For the calculation of the phylogenetic tree programs of PHYLIP 3.63 were used (Felsenstein, 2005). The bootstrap values $<70 \%$ (rounded to whole numbers) based on 1000 replications are indicated at the nodes. Distant clades: SCl-like proteins are found in filamentous fungi (green), Far3/7, Csc4 are found in yeasts (yellow), SIKE-like proteins (blue) and FGFR1OP2-like proteins (purple) are found in the phylum Chordata. The FGOP2 protein, of $D$. melanogaster grouped out because the AA sequence was clearly different compared to the other sequences. 
Results

\subsection{SCl1 proteins are conserved in filamentous ascomycetes}

The phylogenetic tree of STRIPAK-associated proteins containing CC domains showed that S. macrospora $\mathrm{SCl} 1$ and $\mathrm{SCl}$ orthologs from N. crassa, Podospora anserina, Chaetomium thermophilum, Verticillium dahliae, Magnaporthe oryzae, A. nidulans and Aspergillus fumigatus cluster together. To shed light on this, a multiple-sequence alignment of 8 fungal $\mathrm{SCl}$ proteins using MAFFT was performed. With the help of MAFFT, the most conserved region among fungal $\mathrm{SCl}$ orthologs was identified (Figure 11).

The phylogenetic analysis using neighbor joining showed that $\mathrm{SCl} 1$ orthologs from Sordariomycetes or Eurotiomycetes cluster together (Figure S 4 A). Based on the AA alignment, the highest sequence identity was observed between SCl1 of S. macrospora and the $\mathrm{SCl}$ ortholog in N. crassa (93\%). Lower sequence identity was observed between S. macrospora and M. oryzae (56\%) and the lowest sequence identity was observed between S. macrospora and A. fumigatus (20\%) or A. nidulans (18\%) (Figure S 4 B).

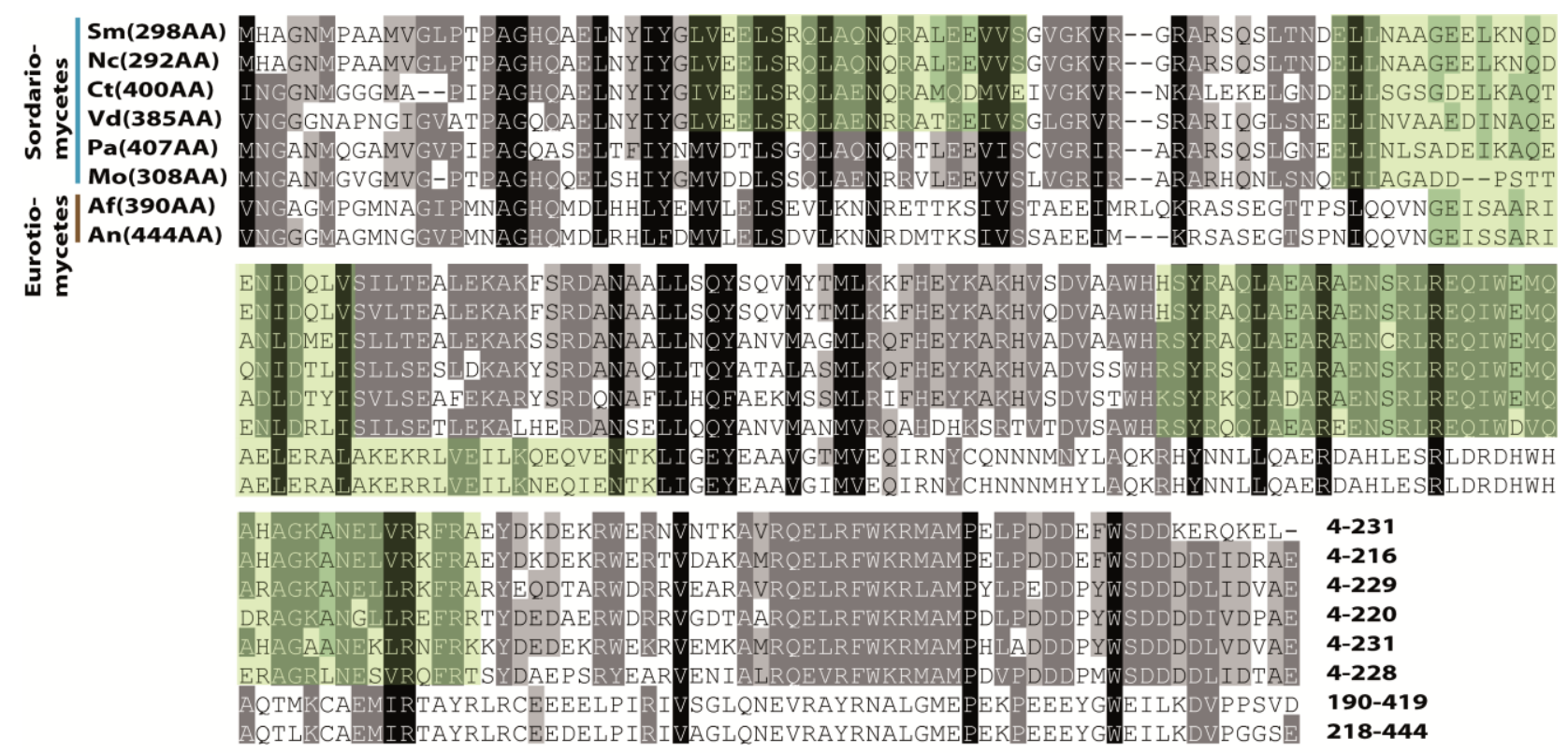

Figure 11: Multiple-sequence alignment of SCI1 proteins in filamentous ascomycetes

The multiple sequence alignment was performed with MAFFT (Katoh \& Standley, 2013) using the aminoacid sequences: Sm, S. macrospora (XP_003349276.1); Nc, N. crassa (XP_960970.1); Ct, C. thermophilum (XP_006696634.1); Vd, V. dahliae (EGY17808.1); Pa, P. anserina (XP_001912083.1); Mo, M. oryzae (XP_003715088.1); Af, A. fumigatus (XP_752657.2); An, A. nidulans (XP_658614.1). The alignment is represented with GeneDoc (Nicholas \& Nicholas, 1997). The overall protein length in amino acids (AA) is presented in brackets and the amino-acid region used for the alignment is indicated at the right hand side at the end of the alignment. Amino acids conserved in all sequences are shaded in black, amino acids conserved in 6 of 8 sequences a shaded in dark gray and amino acids conserved in 5 of 8 sequences are shaded in light gray. Predicted coiled-coil regions are shaded in green. 
However, also highly conserved regions between Sordariomycetes and Eurotiomycetes ( $A$. nidulans and $A$. fumigatus) exist in the $\mathrm{N}$-terminal regions and in the central regions of $\mathrm{SCl}$ proteins. To investigate whether CC domains are also conserved in the 8 fungal SCI proteins COILS/PCOIL and COILS analysis was performed and the predicted CC domains are presented in green in figure 11. The first CC domain was predicted in S. macrospora, N. crassa, C. thermophilum and V. dahliae, the second CC was predicted for all 8 fungal $\mathrm{SCl}$ sequences investigated and the third CC was predicted in $\mathrm{SCl}$ proteins of Sordariomycetes.

\subsection{The sci1 gene is upregulated during sexual development}

S. macrospora propagates sexually by meiotic ascospores, which are generated within fruiting bodies. Sexual development starts at day 2-3 when ascogonia, are formed developing into protoperithecia at day 3-4 and into mature perithecia filled with ascospores at day 6-7 (Pöggeler et al., 2006, Teichert et al., 2014). One complex regulating hyphal fusion and sexual development is the STRIPAK complex in S. macrospora (Beier et al., 2016, Bernhards \& Pöggeler, 2011, Bloemendal et al., 2012, Kück et al., 2016, Nordzieke et al., 2015, Pöggeler \& Kück, 2004). Expression of the STRIPAK genes Smmob3 and pro11 was previously shown to be upregulated during sexual development (Bernhards \& Pöggeler, 2011). Transcript levels of pro45 in vegetative and sexual mycelium and in protoperithecia from day 3-5 had a low abundance in whole transcriptome shotgun sequencing (RNA-seq) analysis (Teichert et al., 2012). In contrast, expression of pro22 increases in sexual development of S. macrospora up to 4-fold after day 4 (Bloemendal et al., 2010). Therefore, the expression level of sci1 in S. macrospora was investigated with quantitative reverse-transcription PCR (qRT-PCR).

The open reading frame (ORF) of the sci1 gene (SMAC_05559) consists of $976 \mathrm{bp}$ and contains one putative intron of $79 \mathrm{bp}$ at the position 242-320. Comparison of genomic and cDNA sequences confirmed the position and the intron size in the scil gene. The genomic sequence of scil and the translated protein sequence are presented in figure S 5. In the qRT-PCR transcript levels of sci1 during vegetative growth were compared to expression levels during sexual growth, after 3, 4, 5, 6 and 7 days. The scil gene was significantly upregulated at day 3 and 7 but significantly downregulated at day 4 . The highest scil transcript level was recorded after day 3, which is the 
developmental stage when ascogonia are formed (Figure 12). The fragments amplified in qRT-PCR with the help of the primer pair RTsci1f/RTsci1r are presented in figure S 6 .

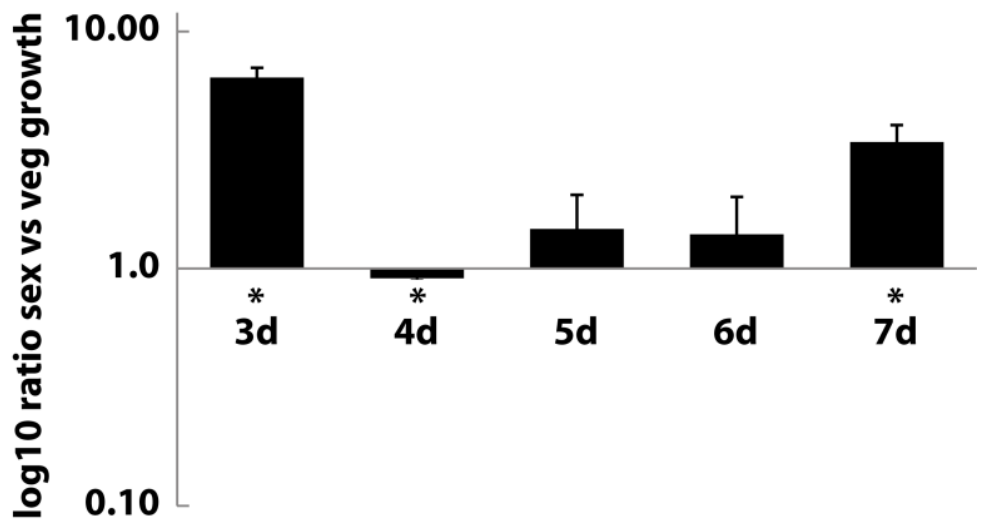

Figure 12: Quantitative reverse-transcription PCR analysis of sci1

RNA was isolated from wt grown in liquid shaking cultures (vegetative growth) and from floating cultures (sexual growth) at day 3-7. qRT-PCR was performed with the primer pair RTsci1f/RTsci1r using cDNA derived from the isolated RNA. Expression of the scil gene was compared at vegetative and sexual growth $(\log 10$ ratio sexual versus vegetative growth) conditions at day 3-7. Mean expression values are derived from 2 independent biological samples measured in triplicates. Standard deviations are given by error bars and stars represent significant results calculated with "REST" (Pfaffl et al., 2002) $p$-values: $3 d, 4 d$ and $7 d<0.001$, 5d: 0.509, 6d: 0.505 .

\subsection{Successful generation of a sci1 deletion strain}

Deletion of STRIPAK components of S. macrospora result in defects in hyphal fusion and sterile strains unable to reproduce sexually (Beier et al., 2016, Bernhards \& Pöggeler, 2011, Bloemendal et al., 2012, Kück et al., 2016, Nordzieke et al., 2015, Pöggeler \& Kück, 2004). Since SCI1 is a putative interaction partner of PRO11 it should be analyzed if a deletion of sci1 results in a similar phenotype as described for S. macrospora strains lacking other STRIPAK components.

For this purpose, a sci1 deletion strain was generated by homologous recombination in the S. macrospora $\Delta$ ku70 strain replacing the scil ORF with a $h p h$ resistance cassette. The $\Delta$ ku 70 strain was used because it is reduced in non-homologous end-joining increasing homologous integration efficiency (Pöggeler \& Kück, 2006). The obtained heterokaryotic mutant strains were crossed to the color spore mutant fus1-1 to reintroduce the $k u 70$ gene and to generate homokaryotic $\Delta$ sci $1_{\text {hyg }}$ strains. The fus1-1 strain resembles the wild type but produces brown spores instead of black spores because of a defect in melanin synthesis (Nowrousian et al., 2012). Black and brown single 
spore isolates (ssi) were selected and deletion of the sci1 gene was confirmed by PCR and Southern blot (Figure 13).

A gDNA
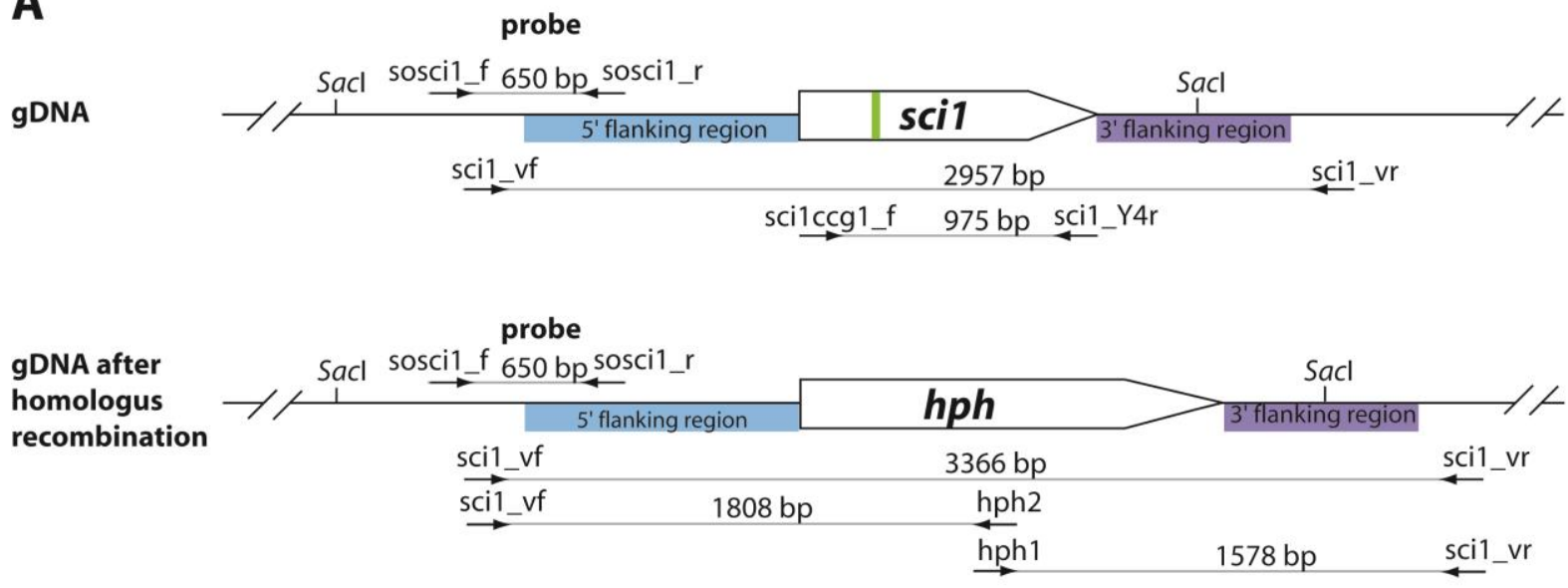

B

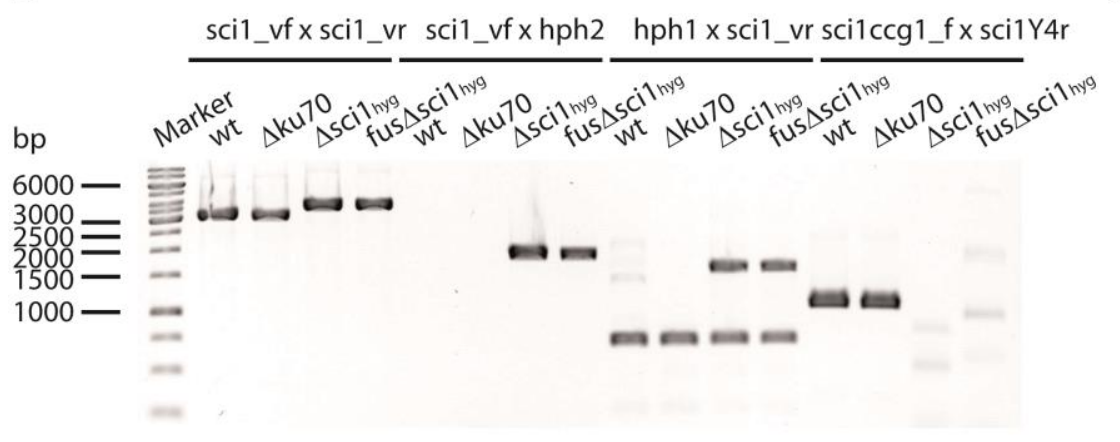

C

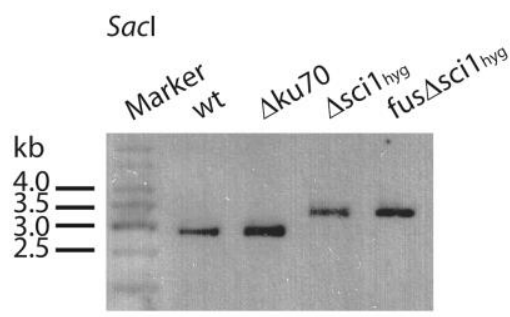

Figure 13: PCR and Southern blot analysis of $w t, \Delta k u 70, \Delta s c i 1_{\text {hyg }}$ and fus $\Delta s c i 1_{\text {hyg }}$

A: Schematic representation of the scil ORF with one intron (green box), 5' flanking region (blue) and 3' flanking region (purple). After homologous recombination the $h p h$ cassette replaced the whole scil ORF. Primer combinations, the corresponding fragment sizes, the probe size and hybridization region are indicated. B: Verification of homologous integration of the $h p h$ cassette into the scil locus using PCR. Genomic DNA was isolated from wt, $\Delta \mathrm{ku} 70, \Delta \mathrm{sci} 1_{\text {hyg }}$ and fus $\Delta \mathrm{sci} 1_{\text {hyg }}$ and tested with given primer combinations. The marker GeneRuler ${ }^{\mathrm{TM}} 1 \mathrm{~kb}$ DNA Ladder (Fisher Scientific) was used for gel-electrophoresis and for the Southern blot. The fragments are given in $\mathrm{bp}$ for the identification of the amplicons or in $\mathrm{kb}$ for the Southern blot. The primer pair hph1/sci1_vr generates a second fragment of about $600 \mathrm{bp}$ for all tested gDNAs, which most likely is caused by unspecific binding of the primers. C: Confirmation of $\Delta$ scil $1_{\text {hyg }}$ and fus $\Delta$ scil $1_{\text {hyg }}$ was done by Southern blot. The isolated gDNA was hydrolyzed with Sacl, the probe and the marker were labeled with alkaline phosphatase, and signals were detected on $\mathrm{X}$-ray films. Signals detected correspond to a fragment size of $2.8 \mathrm{~kb}$ for wt and $\Delta \mathrm{ku} 70$ locus and $3.3 \mathrm{~kb}$ for $\Delta$ sci $1_{\text {hyg }}$ and fus $\Delta s c i 1_{\text {hyg }}$ locus. 


\section{Results}

\subsection{Macroscopic and microscopic analysis of the $\Delta s c i 1_{\text {hyg }}$ strain}

\subsubsection{The sci1 deletion strain is sterile, produces less protoperithecia than the wt and is devoid of hyphal fusion}

For analyzing the impact of sci1 on sexual development, as shown before for pro11, pro22, pro45, Smpp2ac1 and Smmob3, $\Delta$ sci $_{\text {hyg }}$ was investigated macroscopically and microscopically (Beier et al., 2016, Bernhards \& Pöggeler, 2011, Bloemendal et al., 2012, Kück et al., 2016, Nordzieke et al., 2015, Pöggeler \& Kück, 2004).

The wt as well as the sci1 deletion strain were grown on BMM medium and the formation of sexual structures was monitored. The wt, produces first sexual structures at day 2-3, protoperithecia at day 3-4 and mature perithecia filled with ascospores at day 6-7 (Pöggeler et al., 2006, Teichert et al., 2014). In contrast, the $\Delta$ sci $1_{\text {hyg }}$ strain generated ascogonia at day 3 but was unable to produce pigmented protoperithecia and perithecia (Figure 14).

Even after prolonged incubation for 21 days or longer at $27^{\circ} \mathrm{C}$, no pigmented protoperithecia and perithecia could be detected. The $\Delta$ sci $1_{\text {hyg }}$ produced dense areal hyphae, which led to a cotton-like appearance after prolonged incubation. However, the sterile $\Delta$ sci1 hyg phenotype could be complemented with a sci1 full-length construct fused to the egfp sequence, under the control of the endogenous sci1 promoter. Fertility was restored in the $\Delta$ sci $1_{\text {hyg }}:: 5$ 'scilegfp strain which expresses a C-terminally eGFP tagged SCI1 protein ectopically. Similar to the wt the $\Delta$ sci $1_{\text {hyg }}:: 5$ 'scilegf strain produces ascogonia at day 2-3, protoperithecia at day 3-4 and mature perithecia at day 6-7 (Figure $14 \mathrm{~A}$ ).

Not only the development of the $\Delta s c i 1_{\text {hyg }}$ strain is arrested at the stage of unpigmented protoperithecia but also the number of protoperithecia appeared to be reduced. Therefore, protoperithecia with a size of $40-200 \mu \mathrm{m}$ of wt, $\Delta$ sci $_{\text {hyg }}$ and $\Delta$ sci1 $1_{\text {hyg }}:: 5$ 'scilegfp grown on BMMcovered slides were counted per $\mathrm{cm}^{2}$. The experiment was performed in five independent replicates $(n=5)$. The wt produced almost 200 protoperithecia/ $\mathrm{cm}^{2}$ while the number of protoperithecia in the scil deletion strain was reduced by $78.5 \%$ (43 protoperithecia/ $\mathrm{cm}^{2}$ ) compared to the wt strain. The number of protoperithecia in the complementation strain $\Delta$ sci $1_{\text {hyg }}:: 5$ 'scilegfp was reduced by 50\% (100 protoperithecia/ $\mathrm{cm}^{2}$ ) compared to the wt (Figure 14 B). 
Results

A

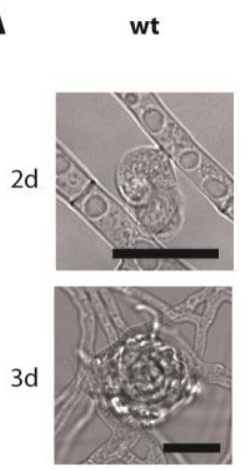

$4 d$

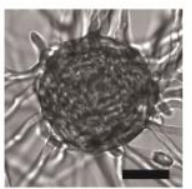

$6 d$

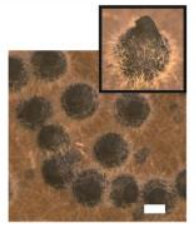

C

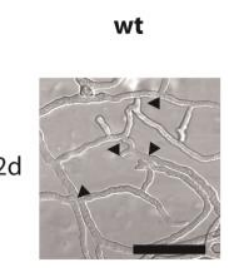

$\Delta$ sci1 hyg
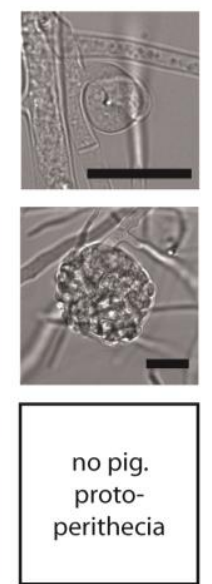

no perithecia
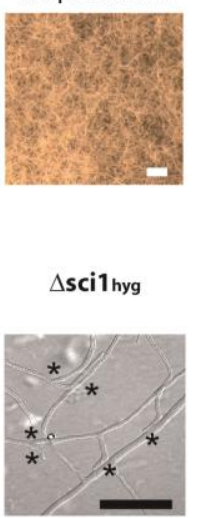

$\Delta$ sci1 hyg::

5'sci1egfp
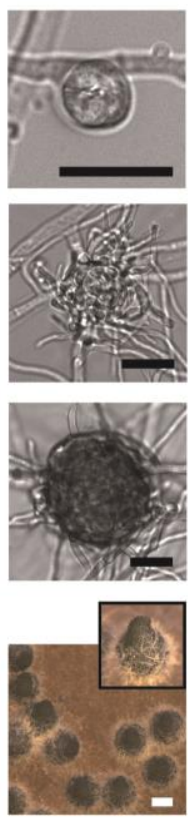

perithecia

$250 \mu \mathrm{m}$

ascogonium

$20 \mu \mathrm{m}$

proto-

perithecium

$20 \mu \mathrm{m}$

pig. protoperithecium

$20 \mu \mathrm{m}$

$\Delta$ sci1 hyg::

5'sci1egfp

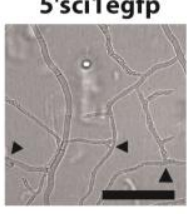

B
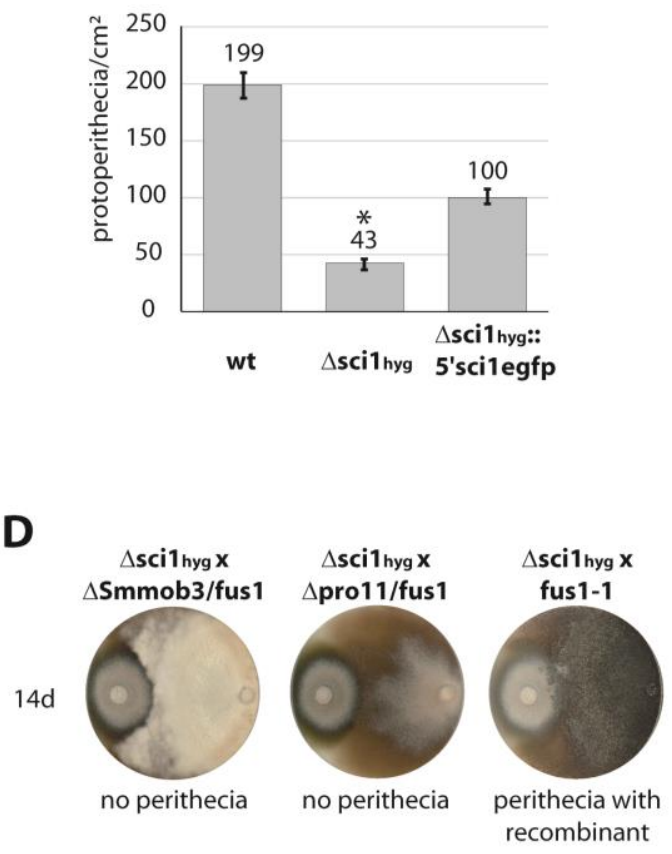

ascospores

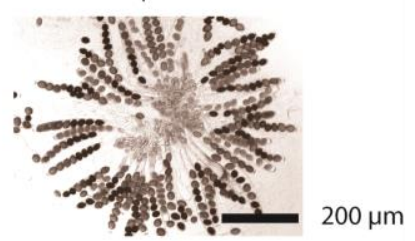

Figure 14: Sexual structures in the wt, $\Delta$ sci $1_{\text {hyg }}$ and $\Delta$ sci1 $1_{\text {hyg }}:: 5$ 'scilegfp

A: Microscopic investigation of the wt, $\Delta$ sci1 $1_{\text {hyg }}$ and $\Delta s c i 1_{\text {hyg }}: 5$ 'scilegfp grown on BMM medium at day 2,3 , 4 and 6 . The wt and the $\Delta$ sci1 $1_{\text {hyg }}:: 5$ 'scilegfp strain develop ascogonia, unpigmented and pigmented protoperithecia and perithecia but the $\Delta$ sci $1_{\text {hyg }}$ develops only ascogonia and unpigmented protoperithecia. For the wt and the $\Delta s c i 1_{\text {hyg }}: 5$ 'scilegfp also a zoomed single perithecium is presented in the upper right corner of the pictures. Pig.: pigmented, d: day B: $W t, \Delta s c i 1_{\text {hyg }}$ and the $\Delta s c i 1_{\text {hyg }}: 5$ 'scilegfp were grown on BMM and protoperithecia with a size of $40-200 \mu \mathrm{m}$ in an area of $1 \mathrm{~cm}^{2}$ were counted in five independent experiments $(n=5)$. The wt produces almost 200 protoperithecia $/ \mathrm{cm}^{2}$ whereas the $\Delta$ sci1 only produces 43 protoperithecia $/ \mathrm{cm}^{2}$. In the partially complemented strain $\Delta$ sci1::5'scilegfp 100 protoperithecia $/ \mathrm{cm}^{2}$ were counted. Error bars represent the standard deviation. Significant results calculated with Student's t-test $(p-$ value $<0.0001)$ are indicated with an asterisk. C: The indicated strains were grown on minimal medium containing soluble starch (MMS) (Rech et al., 2007) covered glass slides for 2 days and hyphal fusion was detected in wt and $\Delta$ sci $1_{\text {hyg }}:: 5$ 'scilegfp but not in $\Delta$ scil $1_{\text {hyg. }}$. Hyphal fusion events are marked with arrows and hyphae making contact without fusion are marked with asterisks. D: The strain $\Delta s c i 1_{\text {hyg }}$ was crossed against the sterile strains $\Delta$ Smmob3/fus1, $\Delta$ pro11/fus1 and the fertile strain fus1-1. Crosses shown were incubated for 14 days. Perithecia with recombinant asci were only detected in $\Delta s c i 1_{\text {hyg }} \times$ fus1-1 crosses. Scale bars as indicated. 


\section{Results}

Although, the 5'scilegfp construct was able to complement the sterile $\Delta$ scil $1_{\text {hyg }}$ phenotype it only partially complemented the reduced protoperithecia phenotype, an effect which might be caused by ectopically integration of the 5'scilegfp construct.

S. macrospora strains without STRIPAK genes pro11, Smmob3, pro22, pro45, and Smpp2ac1 are devoid of hyphal fusion (Bernhards \& Pöggeler, 2011, Bloemendal et al., 2012, Bloemendal et al., 2010, Nordzieke et al. , 2015, Pöggeler \& Kück, 2004, Kück et al., 2016, Beier et al., 2016). Therefore, it was of interest if this is also true for $\Delta$ sci $1_{\text {hyg. }}$

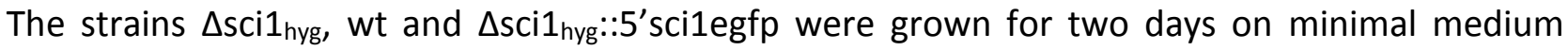
containing soluble starch (MMS) and were investigated microscopically (Rech et al., 2007). In contrast to the wt and the complemented strain, $\Delta$ sci1 hyg was not capable to form hyphal fusion "bridges". Hyphae of $\Delta \mathrm{sci}_{\text {hyg }}$ made contact with other hyphae but hyphal tip extension did not stop (Figure $14 \mathrm{C}$ ). To analyze if the hyphal fusion defect of the $\Delta$ sci $1_{\text {hyg }}$ strain can be complemented by crossing the sterile and hyphal fusion defective strains in the fus1-1 background, $\Delta$ pro $11 /$ fus 1 , $\Delta$ Smmob3/fus1 were crossed against $\Delta$ sci1 $_{\text {hyg }}$ (Nowrousian et al., 2012). These crosses failed to generate perithecia even after prolonged incubation of 14 days in the contact zone indicating that all three strains $\Delta$ sci $_{\text {hyg, }} \Delta$ pro11/fus1 and $\Delta$ Smmob3/fus1 are hyphal fusion defective.

However, crossing of $\Delta$ sci $_{\text {hyg }}$ against the brown-color spore mutant fus1-1 resulted in hybrid perithecia in the contact zone containing asci with four black and four brown ascospores (Figure 14 D). Thus, the genetic defect of the $\Delta$ sci $1_{\text {hyg }}$ strain can be complemented by a strain, which is capable of hyphal fusion indicating that one wt crossing partner is sufficient to mediate hyphal fusion.

\subsubsection{The vegetative growth rate of the $\Delta s c i 1_{\text {hyg }}$ and the $\Delta s c i 1_{\text {hyg }} \Delta$ pro11 strain is reduced}

As the $\Delta$ sci $_{\text {hyg }}$ strain appeared to grow very slow compared to the wt, the vegetative growth rate of $\Delta$ sci $1_{\text {hyg }}$, wt and the $\Delta s c i 1_{\text {hyg }}: 5^{\prime}$ scilegfp was investigated in race tubes filled with SWG. The growth rate of the $\Delta \mathrm{sci}_{\text {hyg }}$ strain was reduced by $91.5 \%$ ( $3 \mathrm{~mm} / \mathrm{day}$ ) compared to wt (35 mm/day), indicating an involvement of SCl1 in vegetative growth. The vegetative growth rate of the complemented strain was reduced by $24 \%$ ( $28 \mathrm{~mm} /$ day) (Figure $15 \mathrm{~A}$ ).

As SCI1 and PRO11 might interact in the STRIPAK complex of S. macrospora it was of interest to determine the effect of a simultaneous deletion of scil and pro11. Therefore, a double deletion 
strain ( $\Delta$ sci1 $1_{\text {hyg }} \Delta$ pro11) was generated and the vegetative growth rate was investigated. To generate $\Delta$ sci $1_{\text {hyg }} \Delta$ pro11, $\Delta$ pro11 was crossed with a fertile strain fus $\Delta$ sci $1_{\text {hyg }}: 5$ 'scilegfp capable to fuse to $\Delta$ pro11 and hygromycin resistant, nourseothricin sensitive strains were selected to eliminate the ectopically integrated 5'scilegfp construct. The strains were sterile and deletions of pro11 and sci1 were verified by PCR (Figure S 7).

A

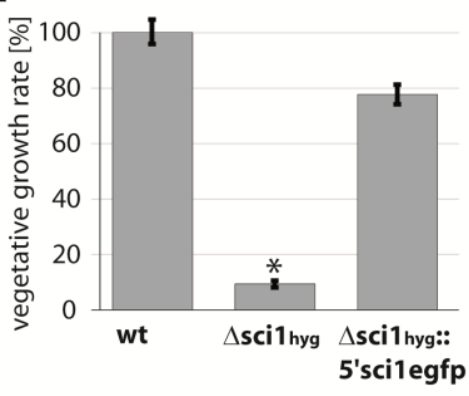

B

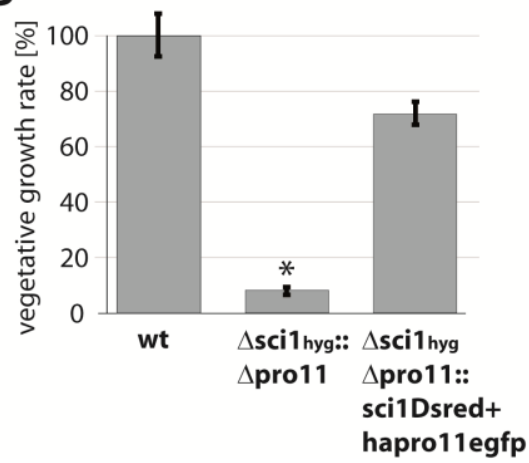

Figure 15: Vegetative growth rates of the wt, $\Delta$ sci $1_{\text {hyg, }} \Delta$ sci $_{\text {hyg }}: 5{ }^{\prime}$ scilegfp, $\Delta s c i 1_{\text {hyg }} \Delta$ pro11 and $\Delta$ sci1 $_{\text {hyg }} \Delta$ pro11::sci1Dsred+hapro11egfp

A: Wt, $\Delta$ sci1 hyg and $\Delta$ sci $1_{\text {hyg }}:: 5$ 'scilegfp and B: wt, $\Delta$ sci $_{\text {hyg }} \Delta$ pro11 and $\Delta$ sci $1_{\text {hyg }} \Delta$ pro11::sci1Dsred+ hapro11egfp were grown in race tubes filled with SWG for 6 days and the vegetative growth rate was monitored. The vegetative growth rate was calculated in $\mathrm{mm} / \mathrm{d}$ and the wt growth rate was defined as 100 $\%$. The standard deviations are represented as error bars. The vegetative growth rate was evaluated from three independent biological replicates performed in triplicates $(n=3)$. Significant results calculated with Student's t-test ( $p$-value A: $\Delta$ sci1 hyg $<0.0001$ and B: $\Delta$ sci $1_{\text {hyg }} \Delta$ pro11 $\left.<0.0001\right)$ are indicated with an asterisk.

The $\Delta$ sci $1_{\text {hyg }} \Delta$ pro11 strain showed a reduced growth rate of $91.8 \%$ ( $3 \mathrm{~mm} /$ day) compared to wt (36 $\mathrm{mm} /$ day) which is similar to the reduced growth rate of $91.5 \%$ of $\Delta$ sci $_{\text {hyg }}$ (Figure $15 \mathrm{~B}$ ). A sci1Dsred construct and a hapro11egfp construct, both under the control of the Pccg1 promoter complemented the sterile phenotype of $\Delta \mathrm{sci} 1_{\mathrm{hyg}} \Delta$ pro11 strain.

Similar as described for $\Delta$ sci1 hyg::5'scilegfp, ectopic integration of the fusion genes hapro11egfp and sci1Dsred into $\Delta$ sci $_{\text {hyg }} \Delta$ pro11 does not fully complement the vegetative growth phenotype of $\Delta$ sci1 hyg $\Delta$ pro11 (Figure 15). However, the constructs 5'scilegfp, hapro11egfp and sci1Dsred were functional because the growth phenotypes of the deletion mutants were partially restored and perithecia were detected. Therefore, $\Delta$ sci $1_{\text {hyg }}$ and the $\Delta$ sci $1_{\text {hyg }} \Delta$ pro11 strain have a comparable reduced vegetative growth phenotype. Comparable, to the findings in this thesis, reduced vegetative growth phenotypes were also reported for deletion of other STRIPAK genes Smmob3, 


\section{Results}

pro11, pro22, pro45 and Smpp2Ac1 (Beier et al., 2016, Bernhards \& Pöggeler, 2011, Bloemendal et al., 2010, Nordzieke et al., 2015, Pöggeler \& Kück, 2004).

\subsubsection{Growth of the $\Delta$ scil $1_{\text {hyg }}$ strain is slightly increased in presence of $\mathrm{H}_{2} \mathrm{O}_{2}$ and $\mathrm{CaCl}_{2}$ or under nitrogen-limiting conditions}

To further characterize the sci1 deletion strain growth in comparison to the wt, pro11, $\Delta$ pro11, and $\Delta$ sci1 $1_{\text {hyg }}:: 5$ 'scilegfp strains was investigated under several stress inducing conditions. All 5 strains were grown for 7 days on SWG stress media supplemented with $0.01 \% \mathrm{H}_{2} \mathrm{O}_{2}$ (oxidative stress), $0.1 \mathrm{M} \mathrm{NaCl}, 0.4 \mathrm{M}$ Sorbitol (osmotic stress), 0.003\% SDS (cell wall stress), and SWG without $\mathrm{KNO}_{3}$ (nitrogen starvation). $\mathrm{Ca}^{2+}$ is involved in hyphal tip growth (Silverman-Gavrila \& Lew, 2001, Takeshita et al., 2017) and Smpp2Ac1 deletion strains are sensitive to high glucose levels (Beier et al., 2016, Son \& Osmani, 2009). Therefore, growth was also tested on SWG containing 0.6 M Glucose (osmotic stress) and SWG supplemented with $0.01 \mathrm{M} \mathrm{CaCl}_{2}$ (osmotic stress/high Ca ${ }^{2+}$ levels) (Figure S 8). Since, the STRIPAK components SmPP2Ac1 and PRO22 were implicated in the cell wall integrity (CWI) pathway, growth of wt, pro11, $\Delta$ pro11, $\Delta$ sci $1_{\text {hyg }}$ and $\Delta$ sci $1_{\text {hyg }}: 5$ 'scilegfp was also tested on SWG medium containing $100 \mu \mathrm{g} / \mathrm{mL}$ calcofluor white (CFW). The color of the SWG medium supplemented with CFW was blue but the wt and the $\Delta$ sci $1_{\text {hyg }}: 5$ 'scilegfp strain fully covered the medium (Figure S 8).

Some stresses did or did not reduce growth of the wt. Almost, all stresses tested reduced growth of the pro11, $\Delta$ pro11, $\Delta$ sci1 $1_{\text {hyg, }} \Delta$ sci1 $1_{\text {hyg }}:: 5$ 'scilegfp compared to growth on SWG (Figure S 8). However, the pro11 and $\Delta$ sci $1_{\text {hyg }}$ strains grew slightly better under nitrogen starvation conditions (SWG without $\mathrm{KNO}_{3}$ ) compared to growth on SWG. No obvious growth reduction for the $\Delta$ pro11 strain was detected on SWG without $\mathrm{KNO}_{3}$ but both strains, $\Delta$ pro11 and the pro11 strain appeared to produce more areal hyphae than on SWG. The $\Delta$ sci1 hyg strain performed better on SWG supplemented with $\mathrm{H}_{2} \mathrm{O}_{2}$ and $\mathrm{CaCl}_{2}$ compared to SWG. Although, $\Delta$ pro11 was reported to grow better under oxidative stress the experiments showed that the strain produced less air mycelium but growth was similar as on regular SWG. Also, growth of pro11 was not reduced in presence of $\mathrm{H}_{2} \mathrm{O}_{2}$ as described before but was reduced by SDS compared to the wt and the other tested strains (Bernhards, 2010) (Figure S 8). 
Results

\subsection{SCl1 oligomerizes and interacts with PRO11 and PRO45 in yeast two-hybrid experiments}

Using affinity purification and LC/MS, SCI1 was identified as putative interaction partner of PRO11 in this study and as putative interaction partner of PRO45 in a former study (Nordzieke et al., 2015). Thus, yeast two-hybrid experiments were performed to investigate direct SCI1-PRO11 and direct SCl1-PRO45 interaction. A further aim was to identify domains, which are required for physical interaction of SCI1 and PRO11 or PRO45 using fragments of SCI1 and PRO11 or PRO45 (Figure 16 A). Full-length sci1 and sci1 fragments were amplified from cDNA and cloned into pGADT7 and pGBKT7 encoding the activation-domain (AD) sequence or the binding-domain (BD) sequence of the transcriptional activator Gal4. Plasmids encoding $A D$ and $B D$ fusion proteins were cotransformed into PJ69-4A and a drop assay was performed. Yeast strains transformed with plasmids encoding the AD fused to the Gal4 interacting protein RanBPM and BD fused to PRO11, PRO45, SCl1 and SCl1 protein fragments grew on SD -Leu, -Trp, -Ade and -His indicating expression of the bait fusion proteins and $\mathrm{Y} 2 \mathrm{H}$ competency (Tucker et al., 2009). All constructs used: pro11, pro45, sci1 or sci1 fragments were not self-activating (Figure $16 \mathrm{~A})$.

To this end, different sci1 constructs fused to the AD or BD sequence were designed and also different pro11 constructs were used (Figure 16 A). Strains expressing full-length BDSCI1 and fulllength ADPRO11 fusion proteins grew on SD -Leu, -Trp, -Ade and -His medium, indicating direct interaction of the two proteins. Unfortunately, BDPRO11 but not BDPRO11 1-110 is self-activating and could not be used to test interaction with ADSCI1 protein derivatives (Bloemendal et al., 2012, Bernhards, 2010) (Figure 16 B).

Strains expressing the fragment ADPRO11 111-281 and BDSCI-FL grew on selective medium, which indicates that the AAs in position 111-281 of PRO11 are required for the interaction with $\mathrm{SCl} 1$ (Figure $16 \mathrm{~B}$ ). On the other hand, the central part of the SCI1 protein, containing the third CC domain and the $A A$ sequences between the second and third CC domain seems to be most important for interaction with PRO11 (Figure 16 A and B). BDPRO11 1-110 and ADPRO11 111-281 did not interact, which was previously described by Bloemendal et al., 2012. However, the oligomerization of PRO11 via its CC domain was confirmed using ADPRO11-FL and BDPRO11 1-110 (Bloemendal et al., 2012) (Figure 16 B). Yeast strains expressing ADPRO11 111-281, and BDSCI1 121-207 or $\mathrm{BDSCl} 1$ 121-298 grew very poorly indicating that the fusion proteins might be folded improperly, are instable or lack a protein region stabilizing interaction between PRO11 and SCI1. 
A

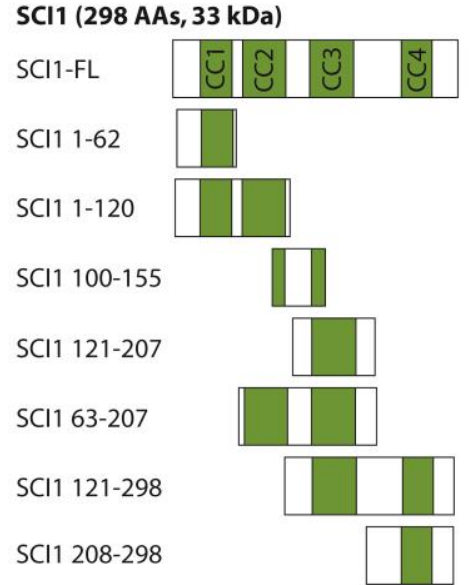

PRO11 (845 AAs, 91 kDa)

PRO11-FL

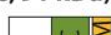

PRO11 1-110

PRO11 111-281

PRO45 (796 AAs, 86 kDa)

PRO45-FL

PRO45DTM

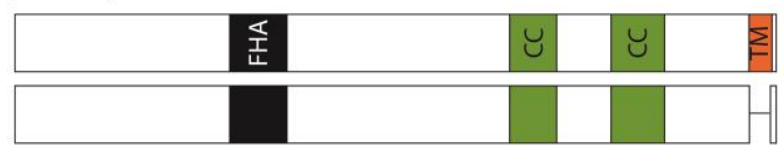

SCI1 208-298

B

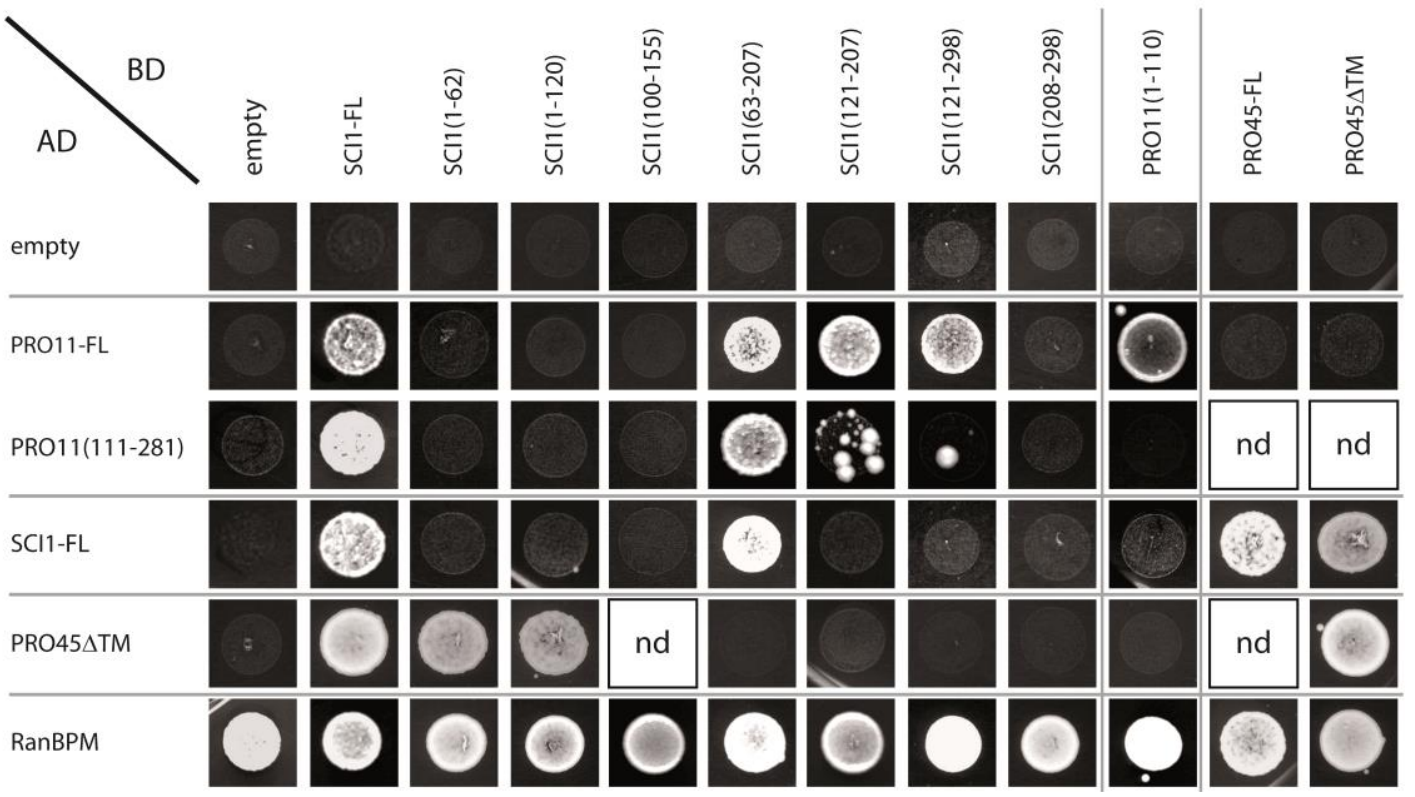

Figure 16: SCI1, PRO11 and PRO45 interact in a yeast two-hybrid analyses

A: The full-length SCl1, PRO11 and PRO45 protein fragments tested in the $\mathrm{Y} 2 \mathrm{H}$ analysis and predicted domains are presented. The position of the AA included in the protein fragments is given and the corresponding predicted domains are indicated. CC: coiled-coil domain (green), CaM: calmodulin-binding domain (yellow), WD: tryptophan-aspartic acid repeats (purple), FHA: forkhead-associated domain (black), TM: transmembrane domain (orange). Representation of PRO11 and PRO45 fragments was modified according to Bloemendal et al. (2012) and Nordzieke et al. (2015). B: Plasmids encoding the Gal4 activating domain (AD) or binding-domain (BD) sequence fused to the respective sci1, pro11 or pro45 gene fragments were cotransformed into PJ69-4A. Yeast strains $(20 \mu \mathrm{L})$ with and $\mathrm{OD}_{600}$ of 0.1 were dropped onto synthetic defined medium without, leucine, tryptophan, histidine and adenine (SD-Leu, -Trp, -Ade, -His). Growth indicates interaction of the tested fusion proteins. RanBPM was used as positive control and indicates $\mathrm{Y} 2 \mathrm{H}$ competency of the BD-constructs. As negative control, plasmid constructs containing corresponding sci1, pro11 or pro45 fragments were cotransformed with empty pGADT7 or pGBKT7 plasmids and show no growth indicating no interaction as expected for the negative controls. nd: not determined in this study. 
Also, interaction of $\mathrm{SCl} 1$ and $\mathrm{PRO} 45$ was verified by $\mathrm{Y} 2 \mathrm{H}$ when full-length $\mathrm{SCl} 1$ was fused to the $\mathrm{AD}$ of the Gal4 transcription factor and either full-length PRO45 or PRO45ATM were fused to the BD

of the Gal4 transcription factor (Figure $16 \mathrm{~A}$ and B) Furthermore, the N-terminal region of SCl1 seems to be most important for interaction with PRO45 (Figure 16 B). Besides the direct interaction of SCI1 with PRO45 shown in this study, oligomerization of PRO45 was confirmed but no direct interaction of PRO11 and PRO45 was detected (pers. com. Steffen Nordzieke) (Figure 16 B). Strains expressing SCI1 full-length proteins ADSCI1-FL and BDSCI1-FL grew on SD -Leu, -Trp, -Ade and -His medium. Thus, the central region of SCI1 (AA 63-207) is required for SCI1 oligomerization (Figure 16 B), which was also shown for its ortholog Far3 in S. cerevisiae (Lai 2011). No growth was detectable with any other construct (Figure 16 B).

\subsubsection{The central region of SCl1 complements the sterile phenotype of $\Delta$ sci $_{\text {hyg }}$}

The central region of $\mathrm{SCl} 1$ was shown to be important for oligomerization of $\mathrm{SCl} 1$ in $\mathrm{Y} 2 \mathrm{H}$ experiments and for interaction with PRO11 (Figure $16 \mathrm{~A}$ ).

Thus, S. macrospora plasmids containing the endogenous sci1 promoter, sci1 fragments encoding the AA region 63-207, 121-207, 121-298 and 208-298 and the heterologous trpC terminator in the pRSnat backbone were transformed into the $\Delta$ sci $1_{\text {hyg }}$ mutant. The fragments used are presented in figure $17 \mathrm{~A}$. Also after prolonged incubation of 3 weeks no perithecia were detected for

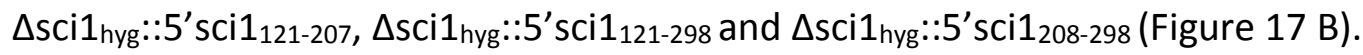

In contrast, the $\Delta$ sci $_{\text {hyg }}:: 5^{\prime}$ sci $1_{63-207}$ strain formed perithecia in limited numbers containing asci with ascospores but only after a prolonged time of 3 weeks after inoculation (Figure 17 B). Thus, the sci1 fragment encoding the central region of SCI1 (AA 63-207) weakly complemented the $\Delta$ scil hyg phenotype. The reduced ability or inability to complement the sterile phenotype might result from improper expression or folding of the truncated proteins. However, the results are consistent with the $\mathrm{Y} 2 \mathrm{H}$ experiments showing $\mathrm{SCl} 1$ oligomerization and the requirement of the central region of SCl1 for interaction with PRO11 (Figure 16 B, Figure 17 B). 
A

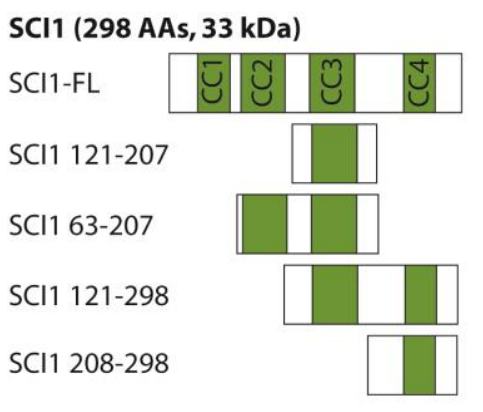

B

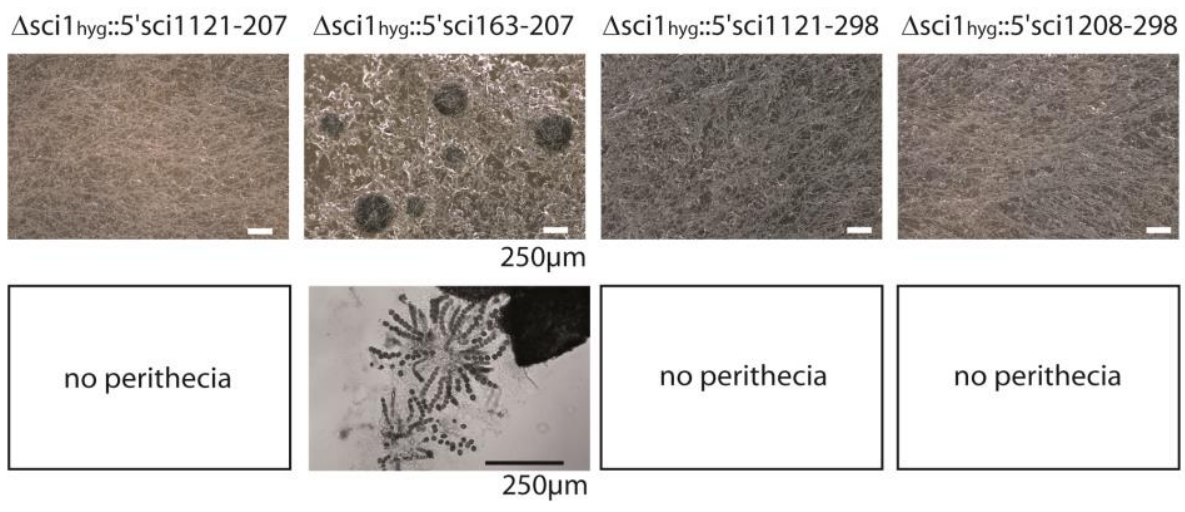

Figure 17: Complementation studies with SCl1 fragments transformed into $\Delta$ sci $1_{\text {hyg }}$

A: Overview of sci1 fragments used for complementation studies in $\Delta$ sci $1_{\text {hyg. }}$ B: The central region of sci1 encoding the AA region 63-207 complemented the $\Delta s c i 1_{\text {hyg }}$ sterile phenotype weakly and mature perithecia were formed. However, perithecia containing asci with ascospores were detected 3 weeks after inoculation on SWG medium. The other SCl1 fragments, 5'sci1 121-207, 5'sci $1_{121-298}$ and $5^{\prime}$ sci $_{208-298}$ did not complement the sterile sci1 deletion strain and perithecia were never detected. Scale bars as indicated.

\subsection{PRO11 was enriched in SCI1eGFP pull-downs}

As SCI1 was identified in PRO11 pull-downs coupled to LC/MS (Figure 8, Table S 1, Table S 3) and both proteins were shown to interact in $\mathrm{Y} 2 \mathrm{H}$ experiments (Figure $16 \mathrm{~B}$ ), $\mathrm{SCl} 1$ was used as bait to search for putative interaction partners in S. macrospora. The total protein content from mycelium of the $\Delta$ sci1 hyg::scilegfp strain was isolated and three independent SCI1eGFP pull-down and LC/MS analyses (SCI1eGFP MS1, SCl1eGFP MS2 and SCI1eGFP MS3) were performed as described in section 2.1.1 and 2.1.2 for PRO11 and SmMOB3 pull-downs (Table 8). In total 1029/887, 1167/893 and 1319/881 proteins were identified with the MaxQuant/Perseus and the Proteome Discoverer ${ }^{\mathrm{TM}}$ software, respectively.

MaxQuant/Perseus data analysis, derived from SCI1eGFP pull-downs and LC/MS is presented in a heat map (Figure $18 \mathrm{~A}$ ). Also, a 2-sample t-test was performed using signal intensities to identify and discriminate significantly enriched candidates in SCI1eGFP pull-downs from unspecific background (Figure $18 \mathrm{~B}$ ). The bait protein SCI1eGFP and PRO11 were highly enriched in all three pull-downs. Simultaneously, dynein heavy chain (SMAC_00761) was enriched with SCI1eGFP in all three pull-down experiments and detected with MaxQuant/Perseus. MaxQuant/Perseus identified PRO22 in all three SCI1eGFP pull-downs. Also, a putative glutamate synthase (SMAC_08095) was enriched but was not detected with the Proteome Discoverer ${ }^{\mathrm{TM}}$ software. Other candidates 
matching the criteria, enriched in at least two pull-downs and not enriched in the negative control were identified and are marked in red in figure $18 \mathrm{~A}$. Two of them, a putative serine-threonine protein kinase (SMAC_02146) and a putative aminotransferase (SMAC_07234) were also identified with the Proteome Discoverer ${ }^{\mathrm{TM}}$ software (Figure 18 ,Table S 4).

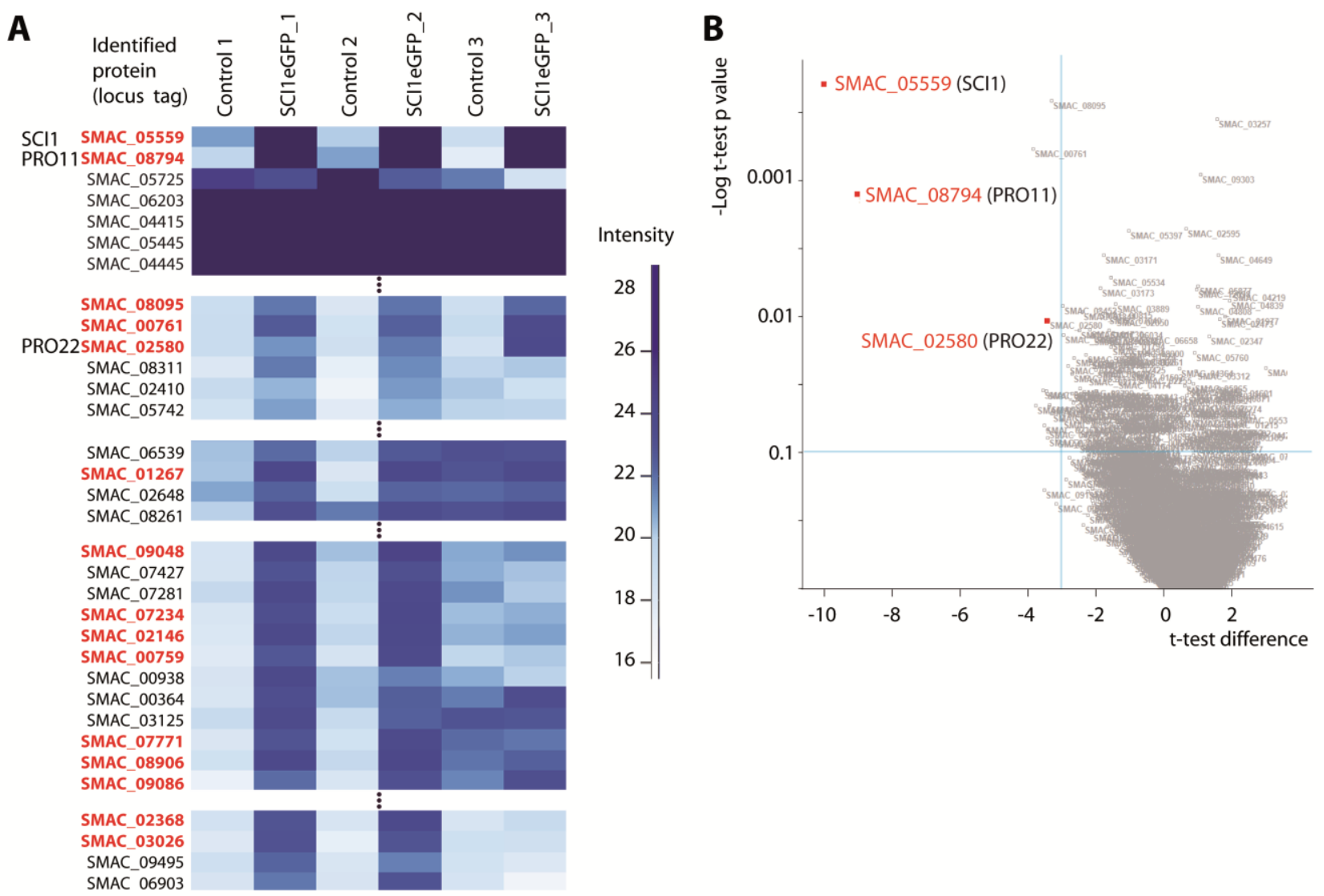

\section{Figure 18: LC/MS data from SCI1eGFP pull-downs coupled to LC/MS and represented in a heat map as well} as in volcano plot

The results of the GFP-Trap using SCI1eGFP as bait and LC/MS are represented in a heat map and in a volcano plot. A: The heat map shows the results of the SCI1eGFP-LC/MS1, 2 and 3 with the corresponding GFP negative control $(1,2$ and 3$)$. Signal intensities correlate with the abundance of the peptides detected. White represents low signal intensities and dark blue indicates high signal intensities. In total, 1029, 1167 and 1319 proteins were identified with MaxQuant/Perseus. Proteins specifically enriched are presented in the heat map. Signal intensities for the bait protein with the locus tag SMAC_05559 (SCI1) were high in all LC/MS experiments. PRO11 (SMAC_08794) was highly enriched in LC/MS1, 2 and 3. PRO22 (SMAC_02580) and dynein heavy chain (SMAC_00761) were also enriched in all three LC/MS experiments. B: The volcano plot represents the results of a statistical 2 sample t-test. Putative $\mathrm{SCl} 1$ interaction partners have a $p$-value $<0.1$ and a t-test difference value of $<-3$ and are marked in red in the heat map.

In contrast, PRO22 was only discovered in one LC/MS experiment with the Proteome Discoverer ${ }^{\mathrm{TM}}$ software. Among others, proteins predicted to be involved in vacuolar transport (SMAC_ 04234 
and SMAC_08299), a putative nucleoporin (SMAC_00744), a putative chitin synthase (SMAC_03109), a putative protein involved in ER to Golgi transport (SMAC_06397) as well as a putative protein involved in transport to and out of the trans-Golgi (SMAC_03125) were identified with the Proteome Discoverer ${ }^{\mathrm{TM}}$ (Table S 4).

\subsection{Functional conservation of SCI1}

Small coiled-coil proteins were detected in STRIPAK complexes of fungi and animals (section 1.1 1.3.1) (Table 1). Although the proteins are barely conserved among phyla it was of interest if their function might be conserved (Figure S 3).

\subsubsection{Mammalian FGFR1OP2 and yeast Far3/7 do not complement the sterile phenotype of $\Delta$ sci $_{\text {hyg }}$}

To investigate the functional conservation of mammalian FGFR1OP2 and yeast Far3/7, plasmids encoding rat and human FGFR1OP2 and S. cerevisiae FAR3 and FAR7 DNA were transformed into the S. macrospora strain $\triangle$ sci1 $_{\text {hyg. }}$. Rat wt Fgfr1op2, human wt FGFR1OP2 and human single nucleotide polymorphism 1 (SNP1) FGFR1OP2 (FGFR1OP2_SNP1) genes derived from cDNA (Lin et al., 2010) were set under the control of the N. crassa ccg1 promotor and the terminator of the anthranilate synthase gene of $A$. nidulans $\operatorname{trp} C$ in the pRSnat background. The plasmids pFGFR1OP2Rn, pFGFR1OP2Hs, pFGFR1OP2Hs_SNP1, pFar3HA and pFar7FLAG were transformed

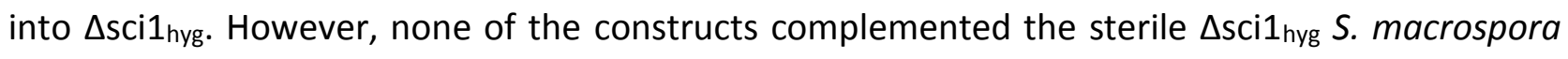
strain.

\subsubsection{SCl1 interacts with Far3 and Far7 but not with Far8 in $\mathrm{Y} 2 \mathrm{H}$ experiments}

Despite the fact that, Far3 and Far7 could not complement the sterile phenotype of $\Delta$ sci $1_{\text {hyg }}$ it was of interest if $\mathrm{SCl} 1$ can interact with the S. cerevisiae proteins Far3, Far7 and with the striatin ortholog Far8 in $\mathrm{Y} 2 \mathrm{H}$ experiments. In S. cerevisiae, the interaction of Far3 and Far7 is the prerequisite for interaction with Far8 (Kemp \& Sprague, 2003, Pracheil \& Liu, 2013). Furthermore, Far3 self-interacts via its central region (Lai et al., 2011). To test interaction of SCI1 with Far3, Far7 and Far8, FAR3, FAR7 and FAR8 were amplified from PJ69-4A gDNA and were cloned into pGADT7. Plasmids described before encoding the SCI1 full-length protein and SCI1 fragments were derived from pGBKT7 (BD) and the combinations used in the $\mathrm{Y} 2 \mathrm{H}$ analyses are presented in figure $19 \mathrm{~A}$. A 
Y2H drop assay was performed and interaction of ADFar3, ADFar7 and ADFar8 fusion proteins was tested with BDSCI1-FL and BDSCI1 protein fragments (Figure $19 \mathrm{~A}$ ). Negative and positive controls are presented in figure $19 \mathrm{~B}$ and figure $9 \mathrm{~S}$.

A

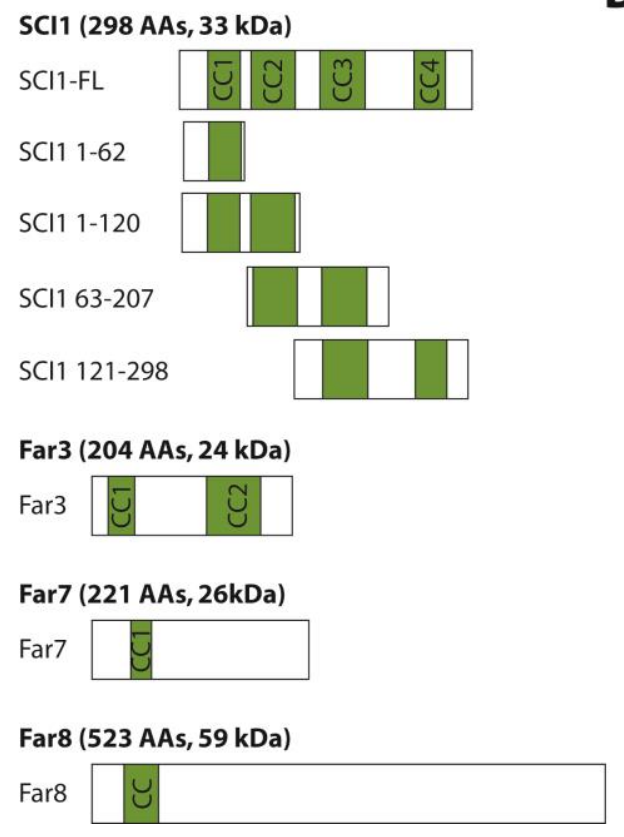

B

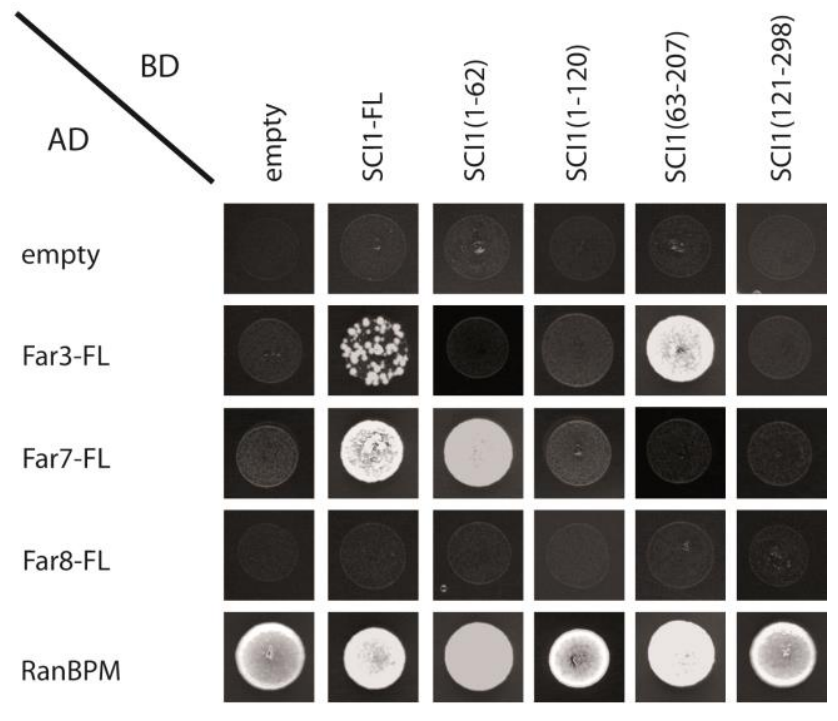

Figure 19: Yeast two-hybrid drop test of SCI1, Far3, Far7 and Far8

A: Constructs of sci1, FAR3, FAR7 and FAR8 were fused to the Gal4 activating domain (AD) or binding-domain (BD) sequence and the respective plasmids were cotransformed into PJ69-4A. CC: coiled-coil domain (green) B: $20 \mu \mathrm{L}\left(\mathrm{OD}_{600}=0.1\right)$ of each yeast strain was dropped on selective medium. Growth of yeasts indicating interaction of the tested fusion proteins was detected on synthetic defined medium without, leucine, tryptophan, histidine and adenine (SD -Leu, -Trp, -Ade, -His). RanBPM was used as positive control and indicates $\mathrm{Y} 2 \mathrm{H}$ competency of the $\mathrm{BD}$ constructs. Constructs containing corresponding fragments were cotransformed with empty pGADT7 or pGBKT7 plasmids and showed no growth indicating no interaction as expected for the negative controls.

Although Far3 and Far7 interact with the striatin ortholog Far8 in S. cerevisiae no interaction could be detected between the ADFar8 and BDSCI-FL or BDSCI1 fragments (Kemp \& Sprague, 2003, Pracheil \& Liu, 2013) (Figure 19 B). Nevertheless, SCl1 and Far7 can interact via the N-terminal region of $\mathrm{SCl} 1$ and $\mathrm{SCl} 1$ and Far3 can interact via the central region of SCl1 (Figure 19 B). Remarkably, the yeast strain expressing the truncated BDSCI1 63-207 fragment and the ADFar3 construct grew better than the strain with the BDSCI1-FL and ADFar3 constructs, which might indicate that the very central region of SCI1 is most important for the interaction with Far3. The 


\section{Results}

SCI1 protein (298 AA) is about one third bigger than the Far3 protein (204 AA). Therefore, the fulllength SCl1 protein might hamper interaction between SCI1 and Far3 (Figure 9, Figure 19).

Taken together, these results are consistent with the results from Lai et al. (2011). The authors reported that Far3 self-associates through its central region. Thus, the function of the central region of SCI1 and Far3 seems to be conserved in S. macrospora and S. cerevisiae and allows interaction of the yeast Far3 and S. macrospora SCI1 protein.

\subsection{SCI1 and PRO11 colocalize at the nuclear envelope}

In fungi, STRIPAK components were found to localize at the nuclear envelope (NE) (Dettmann et al., 2013, Nordzieke et al., 2015, Wang et al., 2010). PRO11 is a STRIPAK complex protein, which interacts with $\mathrm{SCI} 1$. Therefore, fluorescence microscopy was used to investigate localization of the SCI1eGFP fusion protein and synthesis of the SCI1eGFP fusion protein was shown by Western blot

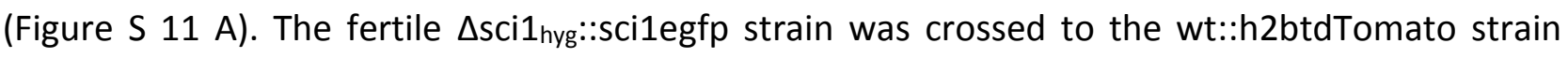
synthesizing TdTomato-tagged histone H2B (Nordzieke et al., 2015), to visualize the nuclei within the hyphae of S. macrospora (Figure 20 A). Also, localization of eGFP-tagged PRO11 in S. macrospora hyphae was performed with fluorescence microscopy (Figure $20 \mathrm{~B}$ ). To do this, the sterile $\Delta$ pro11 strain was complemented with an hapro11egfp construct (Figure S $11 \mathrm{C}$ ) and the fertile $\Delta$ pro11::hapro11egfp strain was crossed with wt::h2btdTomato to visualize the nuclei. The proteins SCI1eGFP and HAPRO11eGFP localized to roundish structures, which were shown to be nuclei using TdTomato-tagged histone H2B (Figure $20 \mathrm{~A}$ and B). In other fungi, striatin orthologs were reported to localize to the NE but no colocalization of striatin orthologs and proteins known to localize to the NE was performed to date (Dettmann et al., 2013, Wang et al., 2010, Zhang et al., 2017). Thus, it was necessary to clarify if SCI1 localizes to the NE.

In U. maydis, the protein pore membrane 152 (POM152) was described to localize to the NE (Theisen et al., 2008). Using BLASTP search the ortholog of UmPOM152 in S. macrospora with the locus tag SMAC_03501 was identified and named SmPOM152. The amino-acid sequence identity between UmPOM152 and SmPOM152 was 27\% with an E-value of $3 \times 10^{-87}$. A plasmid conferring nourseothricin resistance was generated encoding SmPOM152 fused to eGFP. 


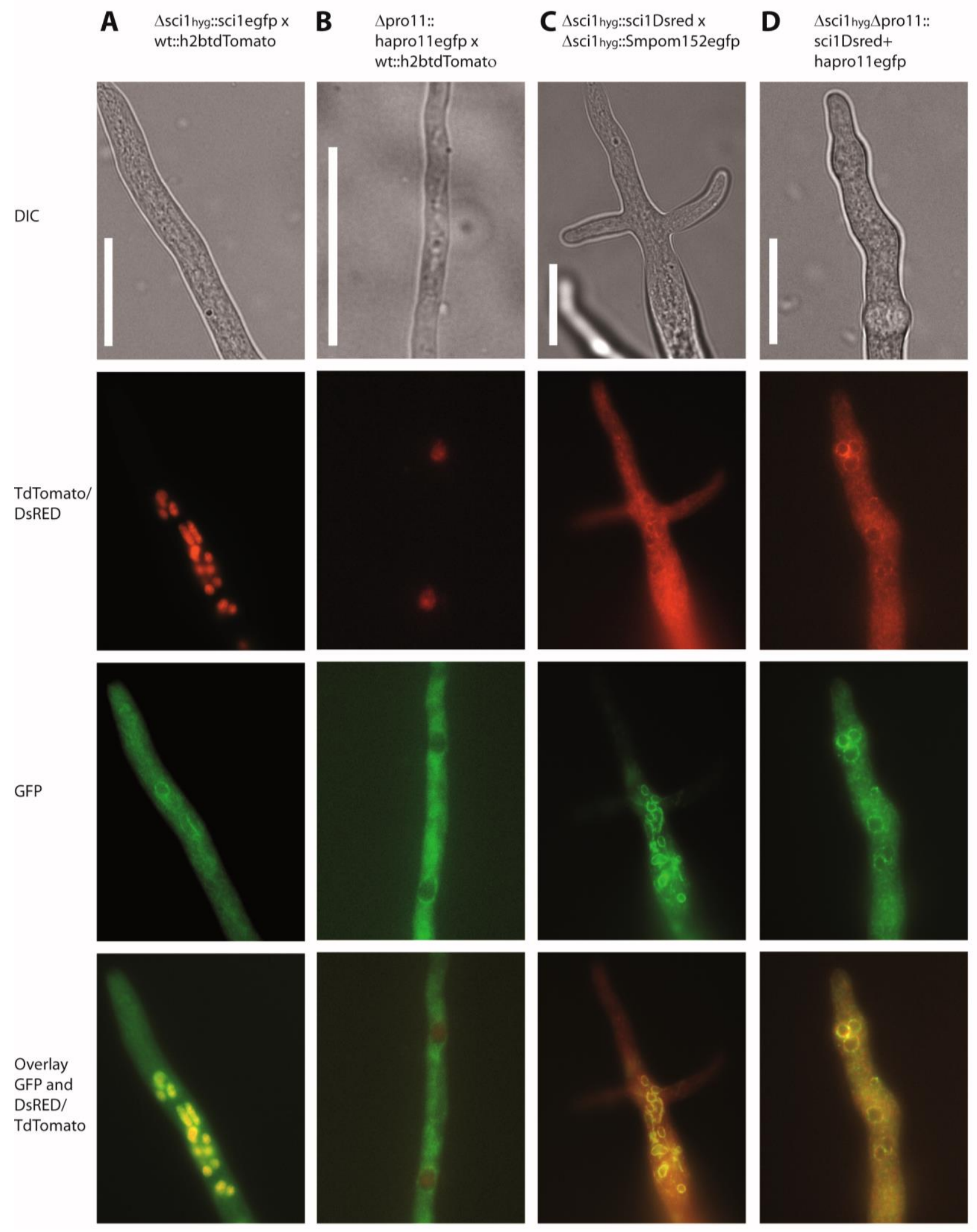

Figure 20: Localization of SCI1 and PRO11 proteins fused to fluorescent tags visualized in vegetative hyphae of $S$. macrospora

A: Localization of SCl1eGFP and H2BTdTomato B: Localization of HAPRO11eGFP and H2BTdTomato C: SCI1DsRED and the nuclear basket protein SmPOM152 fused to eGFP colocalized at the nuclear envelope. D: SCI1DsRED and HAPRO11eGFP colocalize at the nuclear envelope. Scale bars: $20 \mu \mathrm{m}$. 


\section{Results}

The $\Delta$ sci $1_{\text {hyg }}$ strain was transformed with the Smpom152egfp construct under the control of the promoter of the ccg1 gene from $N$. crassa and the synthesized fusion protein SmPOM152eGFP localized to punctiform structures at the NE (Figure 20 C, Figure S $11 \mathrm{E}$ ).

The $\Delta$ sci $1_{\text {hyg }}$ strain was complemented with a sci1Dsred construct expressing C-terminally DsREDtagged SCI1 (Figure S $11 \mathrm{~B}$ ) and crossed to the $\Delta$ sci1 $1_{\text {hyg }}:$ Smpom152egfp strain. Finally, SCI1DsRED was colocalized with SmPOM152eGFP showing that SCI1 localizes at the NE (Figure $20 \mathrm{C}$ ).

Also, PRO11 and SCI1 were colocalized in S. macrospora. The double deletion strain $\Delta$ sci $1_{\text {hyg }} \Delta$ pro11 was complemented with a sci1Dsred and an hapro1legfp construct and nourseothricin and hygromycin resistant strains were selected. The $\Delta s c i 1_{\text {hyg }} \Delta$ pro11::sci1Dsred+hapro11egfp strain was able to produce mature perithecia filled with ascospores indicating that both constructs were functional. The presence of HAPRO11eGFP and SCI1DSRED fusion proteins in the $\Delta$ sci1 ${ }_{\text {hyg }} \Delta$ pro11::sci1Dsred+hapro11egfp strain was verified by Western blot (Figure S $11 \mathrm{D}$ ) and in fluorescence microscopy HAPRO11eGFP and SCI1DsRED colocalized the NE (Figure 20 D, Figure S 12). SCI1eGFP and HAPRO11eGFP also localized to small punctiform structures dispensed in the cytoplasm and to thread-like structures in contact with the NE (Figure S 12).

\subsection{Analysis of cellular components in $\Delta$ sci1}

STRIPAK complexes of mammals, of fission yeast and of the baker's yeast are connected to the nuclear envelope/nuclear trafficking, the endoplasmic reticulum (ER), mitochondria, actin polymerization, septation and endosome trafficking (Baryshnikova et al., 2010, Byers et al., 2009, Chang \& Gould, 2000, Frost et al., 2012, Lisa-Santamaría et al., 2012, Pracheil \& Liu, 2013, Singh et al., 2011). It was also suggested that the STRIPAK in S. macrospora is involved in vesicular transport (Bernhards \& Pöggeler, 2011, Bloemendal et al., 2012). Therefore, fluorescent marker proteins were used to visualize nuclear transport, mitochondria, ER, actin polymerization/septation and endosomal transport in the sci1 deletion background compared to the wt background.

\subsection{1 sci1 deletion does not interfere with nuclear transport}

DAPI stain was applied to visualize nuclei in $S$. macrospora. Unfortunately, DAPI is solved in formaldehyde-containing buffer, which negatively influences the vitality of S. macrospora hyphae, alters appearance of hyphae and hyphal content and finally destroys hyphae. When stained with 
DAPI, hyphae of $\Delta$ sci $_{\text {hyg }}$ appeared to be more fragile than wt hyphae. Also, a reduced amount of formaldehyde in the DAPI stain interfered with hyphal vitality and more rapidly destroyed hyphae of the mutant strain than of the wt.

Therefore, using fluorescence microscopy, nuclei were visualized by TdTomato-tagged histone $2 \mathrm{~b}$ and movement of nuclei was observed in living hyphae of wt S. macrospora. However, no difference was detected between nuclei of wt::h2btdTomato or $\Delta$ sci1 $1_{\text {hyg }}:$ h2 btdTomato (Video 1, Video 2). Nuclei in both strains moved in all directions, forwards as well as backwards. In both strains, nuclei were roundish or oval and sometimes had an elongated structure with a neck-like shape (Figure S 13 A). These nuclei seemed to be drawn by their necks and some crawl through the hyphae (Video 2).

Nuclei in $\Delta$ sci $1_{\text {hyg }}:$ h2 btdTomato hyphae appeared to be herded together but $\Delta$ sci $1_{\text {hyg }}$ shows a strong vegetative growth defect, which might dam up nuclei (Figure S 13 A). Thus, nuclear shape, transport and nuclear distribution seems not to be affected in $\Delta$ scil $_{\text {hyg }}:$ h2btdTomato.

\subsubsection{Mitochondria appeared similar in the wt and in sci1 deletion strains}

Mitochondria were visualized with the fusion construct pMito-DsRED encoding the N-terminal mitochondrial signal sequence of carbonic anhydrase 2 (CAS2) fused to the Dsred sequence (Elleuche \& Pöggeler, 2008). The plasmid pMito-DsRED was transformed into the wt and the $\Delta s c i 1_{\text {hyg }}$ strain and fluorescence microscopy was performed with clones conferring nourseothricin resistance. Mitochondria were similarly distributed within the cytoplasm in hyphae of both strains,

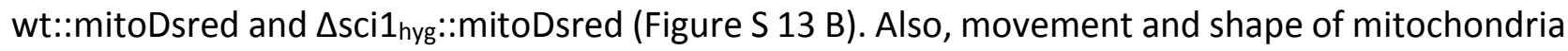
were similar in hyphae of wt::mitoDsred and $\Delta$ sci1 ${ }_{\text {hyg }}:$ mitoDsred (Video 3, Video 4).

\subsubsection{Endoplasmic reticulum in the $\Delta$ sci $_{\text {hyg }}$ background appears normal}

The plasmid pDsREDKDEL encoding the $\mathrm{N}$-terminal signal sequence for cotranslational insertion into the ER from the ER protein PRO41 and the C-terminal ER retention signal KDEL fused to Dsred was transformed into the $\Delta$ sci $1_{\text {hyg }}$ strain and into S. macrospora wt strain to visualize the ER in the hyphae (Nowrousian et al., 2007). Clones with nourseothricin resistance were selected and investigated with fluorescence microscopy. Thread-like structures sometimes surrounding roundish structures, which might be nuclei, were observed in both strains, wt::DsredKDEL and $\Delta$ sci1 hyg::DsredKDEL (Figure S $13 \mathrm{C}$ ). Additionally, punctiform structures were observed, which might be accumulation of missfolded DsREDKDEL fusion proteins (Nowrousian et al., 2007). Taken 


\section{Results}

together, the DSREDKDEL fusion protein localized to thread-like structures in hyphae of wt::DsredKDEL and $\Delta$ sci1 1 hyg::DsredKDEL.

\subsubsection{Actin polymerization and septum formation is not impaired in the sci1 background}

Previously, intercalary septum formation in ascogonia of S. macrospora was shown to be abolished in the pro22 mutant and in the $\Delta$ Smpp2Ac1 mutant (Beier et al., 2016, Bloemendal et al., 2010). Calcofluor white stain (CFW) was applied to stain cell walls and septae in ascogonia of wt, $\Delta$ scil $1_{\text {hyg, }}$ $\Delta$ sci $1_{\text {hyg }}:: 5$ 'scilegfp, pro11 and $\Delta$ pro11. All strains formed intercalary septae in ascogonia and also in vegetative hyphae (Figure $21 \mathrm{~A}$ ).

The STRIPAK complex in mammals and yeasts was reported to be involved in actin polymerization therefore actin polymerization was investigated in S. macrospora (Bai et al., 2011, Baryshnikova et al., 2010, Chen \& Hsueh, 2012, Goudreault et al., 2009, Gupta \& McCollum, 2011, Pracheil et al., 2012). For visualization of F-actin in S. macrospora, the plasmid pAL4-Lifeact (Lichius \& Read, 2009) was transformed into the wt and the $\Delta s c i 1_{\text {hyg }}$ strain and nourseothricin resistant strains were selected. The plasmid pAL4-Lifeact encodes a peptide of 17 AA derived from the actin-binding protein 140 (Abp140) of S. cerevisiae fused to the monomeric red fluorescent protein TagRFP (Riedl et al., 2008, Lichius \& Read, 2009). In filamentous fungi, actin accumulates along the cell wall, polymerizes and contracts to form a ring-like structure (Berepiki et al., 2010) (Figure 21 B).

Septae were formed in young vegetative hyphae of wt::lifeact-tagrfp one after the other as the hyphae grew (Figure 21 B, Video 5). Signals for actin were distributed in a defined region along the cell wall. Than, actin polymerized in a hill-like structure, which most likely represented the contraction of the actin ring preceding septum formation. However, the signal for the TagRFP fluorophore in $\Delta$ sci $_{\text {hyg }}$ background was weaker than in the wt background and bleaching occurred faster than in the wt (Video 6). CFW staining performed with 5\% potassium hydroxide decreased the vitality of hyphae, especially of the $\Delta$ sci $1_{\text {hyg }}$ strain and actin polymerization could not be

properly visualized. F-actin patches and cables were detected in old hyphae of both strains

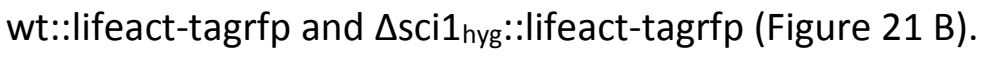


A
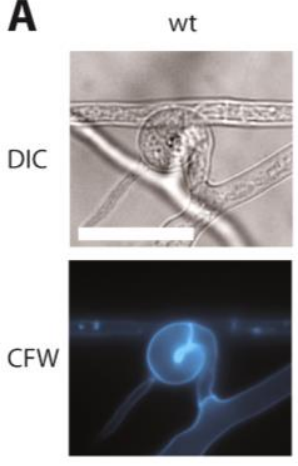

B

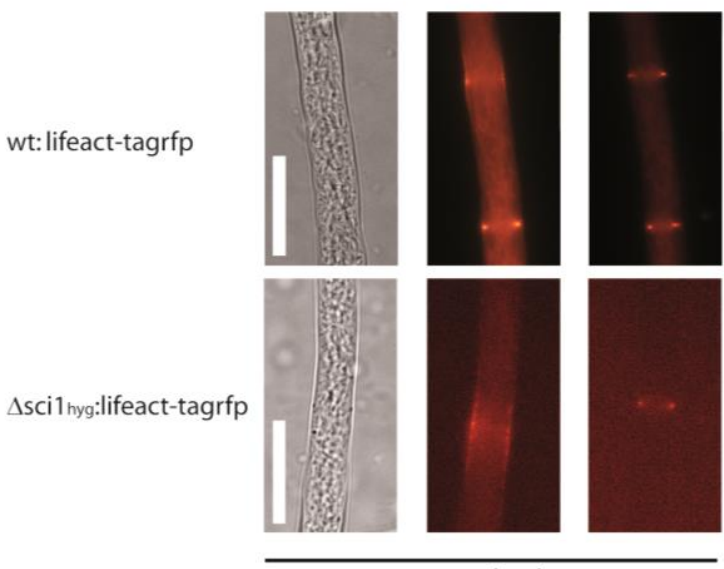

young hyphae
$\Delta$ sci1 hyg:5'sci1egfp
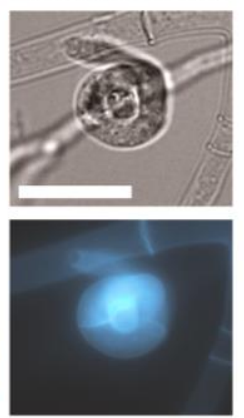

RFP

Actin at

septum
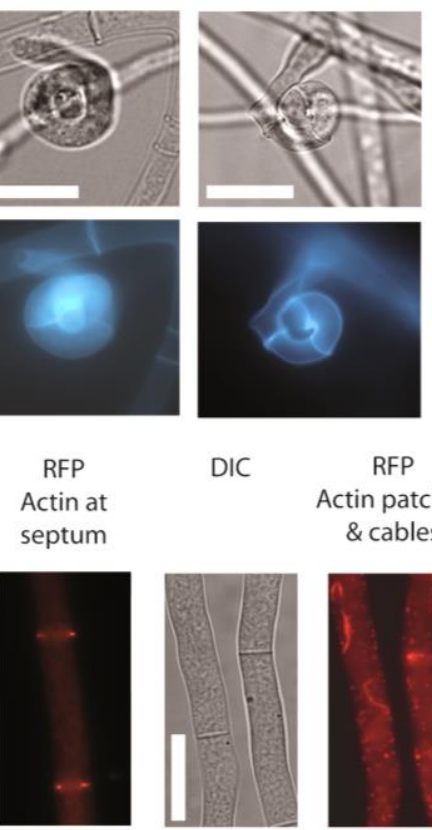

DIC
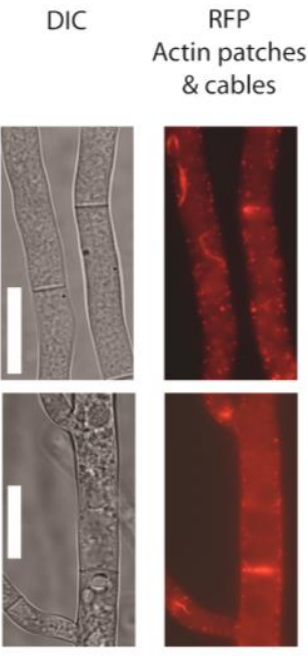

old hyphae

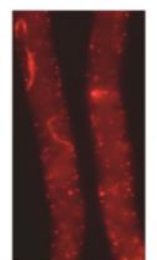

Figure 21: Septation of ascogonia, F-actin polymerization and localization in S. macrospora pro11 and sci1 deletion and wt backgrounds

A: Intercalary septae in ascogonia were visualized with calcofluor white (CFW) stain. B: Actin polymerization in young hyphae visualized by lifeact-TagRFP. Actin accumulates along the call wall, the actin ring contracts and the septum is formed. Also, actin filaments and patches in old hyphae are shown. Scale bars: $20 \mu \mathrm{m}$.

\subsubsection{Visualization of early and late endosomal marker proteins in the wt and the sci1 deletion background}

In mammals, the STRIPAK components striatin and MOB3 (phocein) were reported to be involved in endocytosis and vesicular transport (Baillat et al., 2002, Baillat et al., 2001, Benoist et al., 2006). Similar, in the fungus S. macrospora, in silico analysis suggests a role of SmMOB3 in vesicular traffic and endocytosis (Bernhards \& Pöggeler, 2011). In general, it is thought that endocytosis in filamentous fungi takes place mainly in the hyphal tip. In this model early endosomes (EEs) move to the hyphal tip by kinesin-3 to exchange membrane components with the hyphal tip. Whereas, dynein is thought to mediate retrograde transport of EEs (Steinberg, 2007b). Recently, an early and 


\section{Results}

a late endosomal marker were published for the wheat pathogenic ascomycete Zymospetoria tritici (Zt) (Kilaru et al., 2015).

The small GTPase Rab5 localizes to EEs. EEs mature into late endosomes (LEs) containing the small GTPase Rab7 and finally deliver their content to vacuoles for recycling (Kilaru et al., 2015). The plasmids pHEGFPRAB5 and pHEGFPRAB7 conferring hygromycin resistance were kindly provided by Kilaru et al. (2015) and were transformed into the S. macrospora wt and into the verified $\Delta \operatorname{sci}_{1}$ nat strain (Figure S 14). The $\Delta s c i 1_{\text {nat }}$ strain was sterile and hyphal fusion defective as described for $\Delta$ sci1 hyg (data not shown). Clones were selected for hygromycin resistance and fluorescence microscopy was performed. The fluorescent compound 7-amino-4-chloromethyl-coumarin (CMAC) was used to stain the acidic lumen of vacuoles (Cole et al., 1997, Cole et al., 1998). Vacuolar proteases act on CMAC and stain vacuoles bright blue.

\subsubsection{The early endosome marker eGFPZtRab5 accumulates in hyphae of the $\Delta$ sci $1_{\text {nat }}$ background}

The N-terminally eGFP-tagged EE marker ZtRab5 visualized very small, punctiform structures moving fast in the apical region of the hyphae in the wt::egfpZtrab5 strain. Additionally, mediumsized punctiform structures moved in different speed and in all directions trough the hyphae. Some of them were rather static, whereas others moved rapidly forward and backwards. These punctiform structures merged and split away from each other continuously (Video 7). In the subapical region of the wt::egfpZtrab5 strain signals for eGFPZtRAB5 weakly stained filamentous/tubular structures, which might represent tubular vacuoles (Figure $22 \mathrm{~A}$ ).

Additionally, free eGFP from the eGFPZtRab5 fusion protein (Figure S 16) was detected in the vacuoles of the wt stain (Figure 22 B). In contrast, free eGFP in the wt::egfp control strain is located to the cytoplasm (Figure S 15 A).

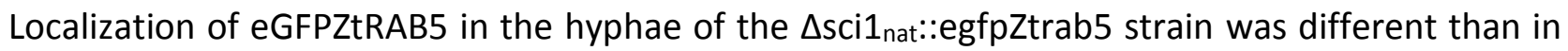
the wt::egfpZtrab5 strain. The signals detected appeared much stronger and three types (1, 2 and 3) of structures were detected in hyphae of $\Delta s c i 1_{\text {nat }}:$ :egfpZtrab5. Tiny vesicles (1) were observed just behind the hyphal tip moving along filamentous-like structures into subapical region of the hyphae. The vesicles moved like beads on a string suggesting transport along tubules or actin filaments (Video 8). 


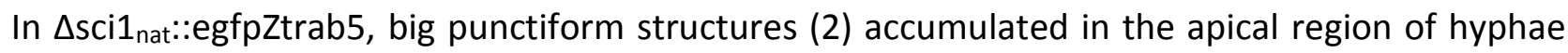
(Figure 22 A) and appeared to incorporate medium sized punctiform structures. These big structures moved marginally with the cytoplasmic flow (Video 9). In $\Delta$ sci $1_{\text {nat }}:$ egfpZtrab5 hyphae also medium sized punctiform structures (3) were detected (Figure $22 \mathrm{~A}$ and B) both moving in different velocities (Video 9). The big punctiform structures in the apical region of $\Delta s c i 1_{\text {nat }}::$ egfpZtrab5 hyphae might represent either accumulated EEs or vesicles fusing to form giant EEs or fusing to other rather static compartments.

A wt::egfpZtrab5
sci1 nat::egfpZtrab5

B wt::egfpZtrab5 $\Delta$ sci1 nat::egfpZtrab5

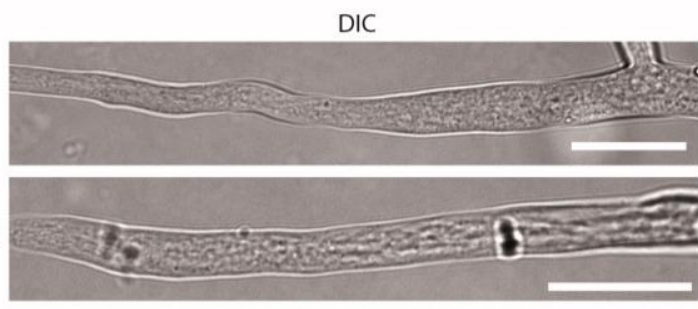

DIC

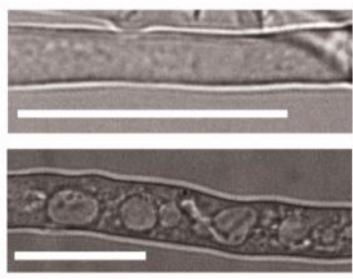

GFP

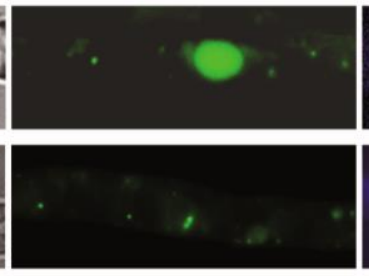

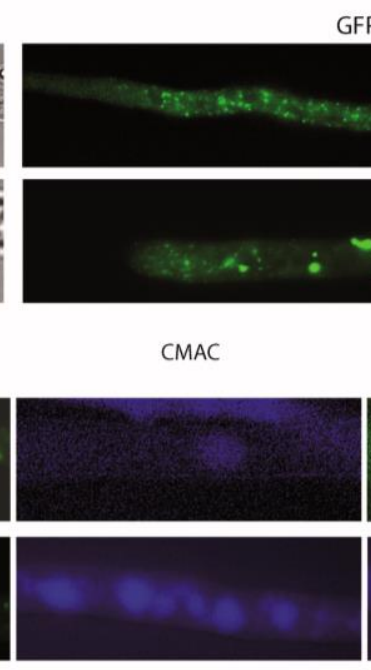

GFP
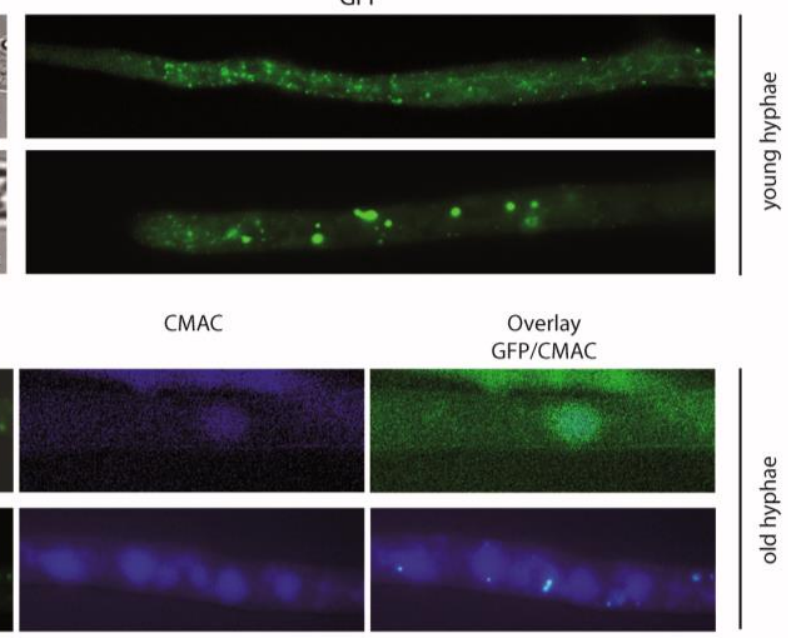

Figure 22: Localization of eGFPZtRab5 in S. macrospora wt::egfpZtrab5 and $\Delta$ sci $_{\text {nat }}$ ::egfpZtrab5 strain Localization of the N-terminally eGFP-tagged ZtRab5 protein was determined in the S. macrospora wt and in the sci1 deletion background. The eGFPZtRab5 fusion protein was localized in A: young hypae and in B: old hyphae. Vacuoles were stained with CMAC. Scale bar size: $20 \mu \mathrm{m}$.

Roundish regions without fluorescence, presumably representing nuclei, were distinguishable in

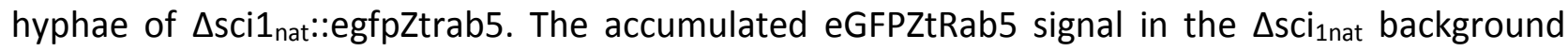
allowed observation of "cloudy" structures moving around the roundish regions (Video 9).

In contrast to the wt::egfpZtrab5 strain no free eGFP was observed in the vacuoles of $\Delta$ sci1 nat::egfpZtrab5 but on several locations fluorescent signals for eGFP appeared like clouds in the cytoplasm next to vacuoles (Figure 22 B). In the control strain wt::egfp free eGFP was localized to the cytosol (Figure S $15 \mathrm{~A}$ ).

To test whether endocytosis is impaired in $\Delta$ sci1 nat::egfpZtrab5 and in other sci1 background strains, hyphae were stained with the amphiphilic dye FM4-64 (Figure S 15 B). FM4-64 stains lipid- 


\section{Results}

rich membranes (Betz et al., 1992, Betz et al., 1996) and is used to investigate endocytosis and endosome to vacuole transport in filamentous fungi (Fischer-Parton et al., 2000, Hoffmann \& Mendgen, 1998, Peñalva, 2005, Steinberg et al., 1998, Vida \& Emr, 1995). Staining of $\Delta$ sci1 and the wt background strains showed that after 20 minutes FM4-64 was distributed within the hyphae (Figure S 15 B). Therefore, endocytosis and vesicle transport is not completely impaired in $\Delta$ scil. Hence, the accumulation of eGFPZtRab5 might indicate a defect in secretion resulting in accumulation of eGFPZtRab5 in $\Delta$ sci $_{\text {nat. }}$. However, the lack of free eGFP in vacuoles of $\Delta$ sci1 nat::egfpZtrab5 but not of wt::egfpZtrab5 might also indicate a defect in degradation processes.

\subsubsection{The late endosome marker eGFPZtRab7 in the $\Delta s c i 1_{\text {nat }}$ background visualizes abnormal vacuolar morphology and behavior}

As EEs mature to late endosomes (LE) marked by the protein Rab5, it was also of interest if LE appearance and transport are altered in the sci1 deletion background. Therefore, a wt strain and a $\Delta s c i 1_{\text {nat }}$ strain were generated, synthesizing the N-terminally eGFP tagged LE marker protein ZtRab7 (Figure S 16).

In wt::eGFPZtRab7 S. macrospora hyphae, eGFPZtRab7 visualized small-punctiform structures in the apical region melting together or splitting away from tubular structures (Figure $23 \mathrm{~A}$ )(Video 10). These, tubular structures most likely represent vacuolar tubules, which were described to exist in S. macrospora (Bloemendal et al., 2010). In filamentous fungi, N. crassa, A. nidulans and Pisolithus tinctorius, tubular vacuoles were described to mediate transport along microtubules in young hyphae (Bowman \& Bowman, 2010, Hickey \& Read, 2009, Bowman et al., 2015).

Rab7 orthologs fused to eGFP in hyphae of $Z$. tritici and $A$. nidulans stained punctiform structures but nothing was reported about tubular structures (Abenza et al., 2012, Kilaru et al., 2015).

Investigating young to middle old wt::eGFPZtRab7 hyphae dynamic vacuoles fusing and splitting again were observed (Figure 23 B). Whereas, middle old hyphae contained spherical and elongated vacuoles with some free eGFP inside (Figure 23C). Spherical vacuoles with free eGFP were detected in middle old to old hyphae (Figure $23 \mathrm{D}$ ). Interestingly, a similar sequence of hyphal components as observed for eGFPZtRab7 in S. macrospora wt::eGFPZtRab7 was visualized by fluorescencetagged calcium transporter proteins, SERCA-type $\mathrm{Ca}^{2+}$ ATPases (NCAs) and the vacuolar protein 
VAM-3 in N. crassa (Bowman et al., 2009). Last but not least, in old wt::eGFPZtRab7 hyphae, small roundish structures without an eGFP signal were detected (Figure $23 \mathrm{E}$ ).

A

wt::eGFPZtRab7

Asci1 nat::eGFPZtRab7

B

wt::eGFPZtRab7

$\Delta$ sci1 nat::eGFPZtRab7

C

wt:eGFPZtRab7

$\Delta$ sci1 nat::eGFPZtRab7

D

wt::eGFPZtRab7

Asci1 nat::eGFPZtRab7

$\mathbf{E}$

wt::eGFPZtRab7

$\triangle$ sci1nat::eGFPZtRab7
DIC
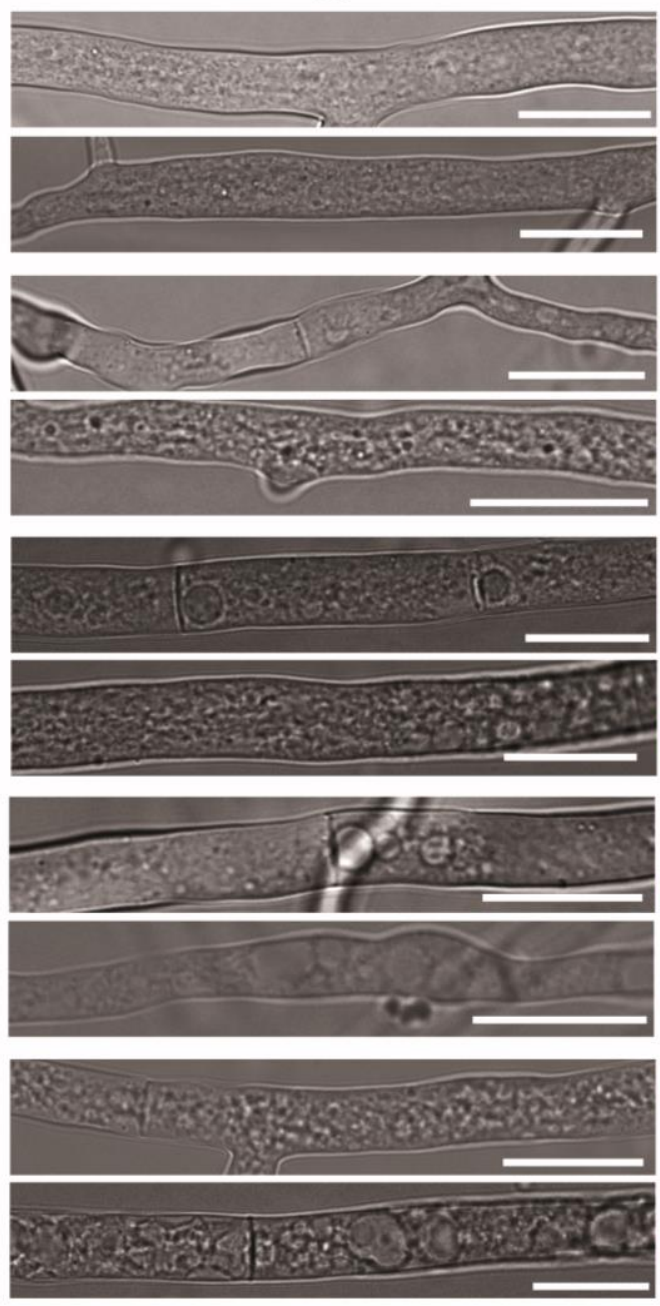

GFP
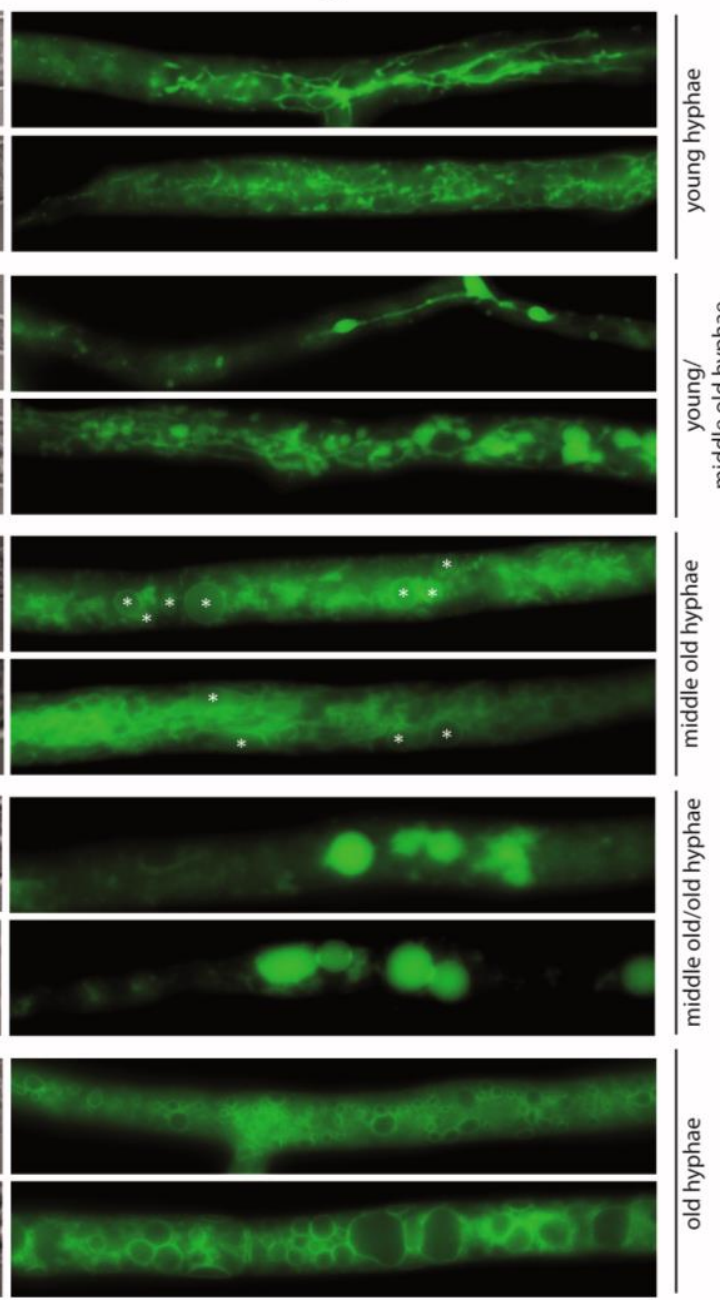

Figure 23: Localization of eGFPZtRab7 in S. macrospora wt::eGFPZtRab7 and $\Delta$ sci $_{\text {nat }}:$ :eGFPZtRab7 strain Localization of the eGFPZtRab7 protein in A: young hyphae (apical region) B: young hyphae, (subapical region) C: middle old hyphae D: middle old to old hyphae and E: old hyphae of $S$. macrospora wt:: ::eGFPZtRab7 and the $\Delta$ sci1 $1_{\text {nat }}:$ :eGFPZtRab7 strain. Vacuolar structures with free eGFP and without free eGFP were detected. Vacuoles with a weak eGFP signal are shown in $\mathbf{C}$ and are marked with asterisks. Scale bar size: $20 \mu \mathrm{m}$

Small and big vacuoles with less or more free eGFP were stained blue using CMAC to stain the acidic lumen of vacuoles. However, small roundish structures without eGFP signals could not be stained by CMAC in hyphae of the wt::eGFPZtRab7 strain (Figure S 17 A). In Z. tritici and A. nidulans LEs visualized by eGFPRab7 associated with CMAC stained vacuoles, which also contained eGFPRab7 


\section{Results}

associated to the membrane (Abenza et al., 2012, Kilaru et al., 2015). At least in A. nidulans, LEs were localized to young hyphae and in both studies LEs were suggested to fuse with vacuoles (Abenza et al., 2012, Kilaru et al., 2015). Therefore, in S. macrospora small-punctiform structures, fusing with most likely tubular vacuoles in young hyphae might represent LEs.

In young hyphae of $\Delta s c i 1_{\text {nat }}:: e G F P Z t R a b 7$, small punctiform structures, presumably LEs, melted to short tubular structures, which appeared more distributed than in the wt (Figure $23 \mathrm{~A}$ and B). Additionally, small vacuoles moving marginally were observed in young hyphae of $\Delta$ sci1 nat::eGFPZtRab7 (Video 11). In P. tinctorius, vacuoles are in close proximity to microtubules and microtubules were considered to determine vacuole morphology and motility (Hyde et al., 1999). Thus, transport of vacuoles along microtubules or connection to microtubules might be disturbed in $\Delta$ sci1 nat::eGFPZtRab7 compared to wt hyphae (Figure 23 A, Video 11). Vacuoles in middle old hyphae were similar to vacuoles detected in wt but the free eGFP signal appeared to be slightly reduced (Figure $23 \mathrm{C}$ ). Also, in middle old to old hyphae spherical vacuoles with free eGFP (reduced compared to wt) were detected showing bright green fluorescence at contact sides of vacuoles (Figure $23 \mathrm{D}$ ). The vacuoles in old $\Delta$ sci $_{\text {nat }}:$ eGFPZtRab7 hyphae appeared bigger and more irregular than in the wt and were brightly stained by CMAC (Figure $23 \mathrm{E}$, Figure S $15 \mathrm{~B}$ ).

In sum, large vacuoles without an eGFP signal were frequently detected in $\Delta$ sci1 nat::eGFPZtRab7 but were rarely seen in the wt background. However, old wt hyphae are located to the center of the mycelium, which is difficult to investigate because of its dense hyphal growth. The regions in which LEs, tubular structures and vacuoles were detected was highly compressed in $\Delta$ sci1 ${ }_{\text {nat }}:$ eGFPZtRab7 strain compared to the wt::eGFPZtRab7 strain. Especially, small vacuolar structures, spherical vacuoles and vacuoles without free eGFP are much closer to the growing tip of the hyphae than in the wt strain (Figure $23 \mathrm{~B}$ ). This pre-aged appearance of young hyphae might be caused by the severe vegetative growth defect of $\Delta$ sci1 or might be caused by disturbed vacuolemicrotubule connection resulting in a slow growth phenotype. 


\section{Discussion}

\section{Discussion}

The conservation of core STRIPAK orthologs (Table 1) between fungi and mammals suggests one or more similar functions among species but accessory proteins in higher eukaryotes indicate additional functions of the respective STRIPAK complexes.

The Far complex in S. cerevisiae synchronizes mating cells allowing enough time for cell to cell fusion before karyogamy takes place and thus regulates sexual reproduction, a process found in higher eukaryotes (Kemp \& Sprague, 2003). The SIN in S. pombe and the Far complex in S. cerevisiae are connected to actin-ring constriction and actin polymerization required for cytokinesis and for cell to cell fusion (Baryshnikova et al., 2010, Pracheil et al., 2012, Singh et al., 2011). Likewise, in the filamentous ascomycete S. macrospora, the SmSTRIPAK complex controls cell fusion and is required for vegetative growth and sexual development (Beier et al., 2016, Bernhards \& Pöggeler, 2011, Bloemendal et al., 2012, Bloemendal et al., 2010, Nordzieke et al., 2015, Pöggeler \& Kück, 2004). However, the underlying molecular mechanisms are unknown. Mature fruiting-body formation is the only way of $S$. macrospora reproduction and generation of sexually derived ascospores is blocked in SmSTRIPAK deletion mutants (Lord \& Read, 2011, Pöggeler et al., 1997, Teichert et al., 2014). Thus, the presence of a functional STRIPAK complex is essential durring development of S. macrospora.

S. macrospora, likewise the fruit fly and mammals contains core STRIPAK components and associated proteins, indicating that the SmSTRIPAK might be involved in many different processes (Table 1). In D. melanogaster, the dSTRIPAK regulates apoptosis and controls tissue size as well as dorsal closure in embryos (Chen et al., 2002). In mammals, the STRIPAK complex functions in cellcycle control, apoptosis, cell migration, vesicular traffic, development of neurons, and was connected to cardiac diseases, neuronal diseases and cancer (Bai et al., 2011, Baillat et al., 2001, Bartoli et al., 1999, Breitman et al., 2008, Castets et al., 1996, Chen \& Hsueh, 2012, Grand et al., 2004, Hwang \& Pallas, 2014, Ishikawa et al., 2012, Lin et al., 2010, Meurs et al., 2010). Therefore, investigating STRIPAK complex components in S. macrospora will help to understand the cellular functions in higher eukaryotes, controlled by the STRIPAK complex.

In this study, the yet undescribed protein SCI1 was enriched in PRO11 pull-downs and was identified by LC/MS. A main question of this study was, if SCI1 is a STRIPAK complex component in S. macrospora. Also function and subcellular localization of SCI1 was investigated and the spatialtemporal localization of cellular components in the sci1 deletion strain with the wt were compared. 


\section{Discussion}

This study connects the SmSTRIPAK to the Golgi and vesicular traffic arguing for a dynamic SmSTRIPAK complex mediating very complex processes like hyphal fusion and sexual development in S. macrospora.

\subsection{SCl1, a new PRO11 interaction partner was identified in S. macrospora}

In mammals, striatins act as $\mathrm{B}^{\prime \prime \prime}$ regulatory subunits of the protein phosphatase PP2A and as scaffolding proteins in the STRIPAK complex to bring STRIPAK components together (Goudreault et al., 2009, Moreno et al., 2000). In the STRIPAK complex of S. macrospora (SmSTRIPAK), the striatin ortholog PRO11 seems to be a central component of the SmSTRIPAK complex (Bernhards \& Pöggeler, 2011, Bloemendal et al., 2012, Pöggeler \& Kück, 2004). Deletion of pro11 results in a strain producing only rudimentary ascogonia, and representing the most severe phenotype compared to strains lacking other SmSTRIPAK complex genes, indicating a central role in the function of the SmSTRIPAK complex (Bernhards, 2010). PRO11 directly interacts with the kinase activator SmMOB3 (Bernhards \& Pöggeler, 2011, Bloemendal et al., 2012). Therefore, PRO11 and SmMOB3 were used as baits in pull-down experiments coupled to LC/MS to identify potential interaction partners of the two SmSTRIPAK complex members.

To analyze the LC/MS data, two programs MaxQuant/Perseus and the Proteome Discoverer ${ }^{\mathrm{TM}}$ were applied. MaxQuant/Perseus allows identification of peptide precursors, but label-free quantification (LFQ) analysis and independent replicates generate data with different qualities.

The samples applied to LC/MS were very heterogeneous and more proteins were identified with MaxQuant/Perseus using LFQ than with the Proteome Discoverer ${ }^{\mathrm{TM}}$ (Table 11, Table 12).

The criteria were stringent to distinguish between enriched proteins and unspecific background. In sum, analysis of the LC/MS data with MaxQuant/Perseus identified known interaction partners of PRO11 and SmMOB3 and the new putative PRO11 interaction partner SCI1 with very high confidence (Figure 8, Figure S 1).

The LC/MS data was also evaluated based on the sum of spectral counts using the Proteome Discoverer ${ }^{\mathrm{TM}}$ software. However, in complex samples a peptide precursor might not be selected by the mass spectrometer for fragmentation and a protein might not be detected with the Proteome Discoverer ${ }^{\mathrm{TM}}$ software. In general, less proteins were identified with the Proteome Discoverer $^{\mathrm{TM}}$ than with MaxQuant/Perseus software (Table 11, Table 12) and the criteria were less 
stringent. Data from each pull-down/LC/MS replicate (HAPRO11 and HAPRO11eGFP (MS1-3) or FLAGSmMOB3 and SmMOB3eGFP (MS1-3)) was evaluated with the Proteome Discoverer ${ }^{\mathrm{TM}}$ according to the criteria mentioned in Materials and Methods, section 1.6.11.4. In a previous study, pull-downs with the TAP-tagged SLMAP ortholog PRO45 and LC/MS analysis PRO11, SmMOB3 and SCI1 were enriched with very high sum of spectral counts (Nordzieke et al., 2015). Both studies indicate, that SCI1 might be an uncharacterized SmSTRIPAK complex component. In addition, proteins connected to vesicular transport, kinases, a nucleoporin and a small GTPase of the Ras family were identified. However, comparison of enriched proteins identified, SCl1 as most promising potential interaction partner of PRO11. Therefore, the function of SCI1 was analyzed in detail (Table S 1, Table S 2, Table S 3).

\subsection{SCl1 is a small protein containing coiled-coil domains}

Up to now, no sci gene was described or investigated in filamentous fungi. In S. macrospora, sci1 with the locus tag SMAC_05559 encodes a protein comprising 298 AAs (Figure S 5, Figure 9). Beside the four coiled-coil (CC) domains no other domain could be predicted for SCI1 (Figure 9). A CC domain is characterized by a repetitive heptade pattern $($ HPPHPPP) $n$, where $H$ represents a hydrophobic amino acid and $P$ represents a polar amino acid. Coiled-coils are amphiphilic $\alpha$-helices interacting with each other through hydrophobic interactions between the helices (Crick, 1952). They can act as spacer between functional domains but can also mediate protein-protein interactions (Crick, 1952, Munro, 2011, Surkont \& Pereira-Leal, 2015, Zhang et al., 2009). Small proteins containing CC domains are associated with the STRIPAK complex (Table 1) but it is not known if they mediate protein interactions.

The human STRIPAK complex associates mutually exclusively with either CTTNBP2 or with SLMAP and the CC-domain containing proteins SIKE and FGFR1OP2 (Goudreault et al., 2009). However, it is not known if CTTNBP2 orthologs exist in fungi. SIKE is assumed to be involved in the systemic response against infection and FGFR1OP2 seems to be required in wound healing, which involves cell fusion (Huang et al., 2005a, Lin et al., 2010). The small CC proteins FGOP2 in D. melanogaster

and Csc4 in S. pombe are described as STRIPAK complex components, but are neither intensively studied in the fruit fly nor in fission yeast (Ribeiro et al., 2010, Singh et al., 2011). The CC proteins, 


\section{Discussion}

Far3 and Far7 of S. cerevisiae were shown to be required for the pheromone-mediated G1 arrest (Kemp \& Sprague, 2003, Lai et al., 2011, Pracheil \& Liu, 2013).

SCI1 was identified in PRO11 pull-downs in this study (Figure 8, Table S 1) and was also previously enriched in PRO45 (SLMAP ortholog) pull-downs (Nordzieke et al., 2015) indicating that SCI1 is a STRIPAK complex component in S. macrospora. All described small CC proteins associated with the STRIPAK contain one to four CC domains but are barely conserved by sequence (Figure 9, Figure 10). Therefore, BLAST searches cannot be applied to identify the small CC proteins, which are associated with the STRIPAK complex between distantly related species. However, phylogenetic analysis showed that STRIPAK-complex associated small CC proteins are conserved within the phyla. Vertebrates contain FGFR1OP2- and SIKE-like proteins, invertebrates contain FGOP2-like proteins, yeasts contain Far3/7- and Csc4-like proteins and filamentous fungi contain SCl-like proteins (Figure 10).

The number of predicted CC domains and the length of the proteins vary between species indicating that these proteins and the STRIPAK complex might fulfill diverse functions in the respective organisms. In filamentous Ascomycetes such as S. macrospora, N. crassa, P. anserina, C. thermophilum, V. dahliae, $M$. oryzae, A. nidulans, and $A$. fumigatus $\mathrm{SCl}$-like proteins are conserved by sequence but were not yet investigated (Figure 11).

\subsection{Deletion of sci1 causes sterility and prevents hyphal fusion in S. macrospora}

The S. macrospora wt reproduces via sexually formed ascospores (Lord \& Read, 2011, Pöggeler et al., 1997) and loss of sci1 leads to a sterile strain unable to produce pigmented protoperithecia and perithecia as well as ascospores (Figure $14 \mathrm{~A}$ ). Also, no hyphal fusion was detected in $\Delta$ sci $1_{\text {hyg }}$ and protoperithecia formation was highly reduced (Figure $14 \mathrm{~B}$ and C). The phenotype of the $\Delta$ sci $1_{\text {hyg }}$ strain is similar to other $S$. macrospora SmSTRIPAK complex mutants ( $\Delta$ pro11, $\Delta$ pro22, $\Delta$ Smmob3, $\Delta$ Smpp2ac1 and $\Delta$ pro45), which are also sterile and hyphal fusion defective (Beier et al., 2016, Bernhards \& Pöggeler, 2011, Bloemendal et al., 2012, Bloemendal et al., 2010, Nordzieke et al., 2015, Pöggeler \& Kück, 2004). Similarly, deletion of STRIPAK complex genes ham-2, ham-3, ham-4, mob-3 and ppg1 (pp2Ac) in N. crassa results in hyphal fusion defective strains, which are female sterile and unable to form protoperithecia (Fu et al., 2011, Maerz et al., 2009, Simonin et al., 2010, Xiang et al., 2002). In other filamentous fungi, such as A. nidulans, F. verticillioides, F. graminearum, 
F. virguliforme, C. graminicola, and E. festucae STRIPAK complex components are required for hyphal fusion, mycelium growth, sexual development and additionally virulence or symbiotic interaction (Shim et al., 2006, Wang et al., 2010, Green et al., 2016, Wang et al., 2016, Islam et al., 2017, Zhang et al., 2017).

However, a fusion competent S. macrospora strain can fuse to the $\Delta$ sci $1_{\text {hyg }}$ fusion-defective strain (Figure $14 \mathrm{D}$ ) which might indicate that recognition, fusion or both do not necessarily require regulated cell communication of the fusion partners. In N. crassa, fusion of hyphae happens as follows: hyphae make contact, extension of the hyphal tip stops, material required for adhesion is secreted, adhering hyphae swell, the cell wall and adhesive material is removed, both plasma membranes make contact and the fusion pore is formed (Hickey et al., 2002). The fact that extension of $\Delta$ sci $_{\text {hyg }}$ hyphae does not stop upon contact with other hyphae but wt hyphae can fuse to $\Delta$ sci $1_{\text {hyg }}$ hyphae it is tempting to speculate that deletion of scil might affect secretion from S. macrospora hyphae. Thus, $\Delta$ sci $1_{\text {hyg }}$ hyphae might not recognize each other and are unable to attach to each to other or both. However, it remains a very interesting task for the future to test secretion in $\Delta$ sci $_{1 \text { hyg }}$ compared to the wt.

In addition, vegetative growth in filamentous fungi is connected to exocytosis and vesicular transport (Bartnicki-Garcia, 2002, Steinberg, 2007a) and LC/MS analyses of PRO11, SmMOB3 and SCl1 pull-downs, identified proteins related to transport (Table S 1, Table S 2, Table S 3, Table S 4). Similarly to $\Delta$ pro11 (Bernhards, 2010) the $\Delta$ sci $_{\text {hyg }}$ strain is highly reduced in vegetative growth (Figure $15 \mathrm{~A}$ ) which is also true for $\Delta$ sci1 hyg $\Delta$ pro11 (Figure $15 \mathrm{~B}$ ). Thus, the strongly reduced growth rate of the sci1 deletion background might indicate impaired vesicular transport (section 3.9 and 3.9.2).

Growth test of $\Delta$ sci $1_{\text {hyg }}$ in presence of several stresses were performed to further investigate the scil deletion phenotype. Growth of $\Delta$ scil $_{\text {hyg }}$ was compared to the wt, pro11, $\Delta$ pro11 and the complemented strain $\Delta$ sci1 $1_{\text {hyg }}: 5^{\prime}$ scilegfp. Although, deletion of striatin orthologous genes were reported to be sensitive to high glucose levels and CFW induced cell wall integrity stress, all strains tested in this study were reduced in presence of high sugar levels and CFW compared to growth on SWG (Beier et al., 2016, Son \& Osmani, 2009) (Figure S 8). In contrast, presence of oxidative stress $\left(\mathrm{H}_{2} \mathrm{O}_{2}\right)$, nitrogen starvation and presence of high $\mathrm{CaCl}_{2}$ levels slightly increased growth of $\Delta$ sci1 $1_{\text {hyg }}$ (Figure S 8). Nitrogen starvation in yeasts and S. macrospora induces autophagy, therefore it can be speculated that induction of autophagy might be negatively influenced in the $\Delta$ sci $1_{\text {hyg }}$ 
strain (Takeshige et al., 1992, Voigt \& Pöggeler, 2013, Werner et al., 2016). Additionally, improved growth of the sci1 deletion strain in presence of high $\mathrm{CaCl}_{2}$ levels might indicate that calcium supply or a calcium mediated process required for hyphal tip growth might be disturbed (Kim et al., 2012, Silverman-Gavrila \& Lew, 2001, Takeshita et al., 2017). In A. nidulans, Ca ${ }^{2+}$ causes depolymerization of actin and subsequently fusion of accumulated secretory vesicles with the plasma membrane. Therefore, it tempting to speculate that sci1 deletion might cause a defect in transport of secretory vesicles, exocytosis or both.

\section{4 sci1 is up-regulated during sexual development}

In S. macrospora, cell differentiation, cell wall modulation and formation of three-dimensional fruiting bodies requires tight regulation involving the genes of the SmSTRIPAK complex (Teichert et al., 2014). The pro11 deletion strain produces rudimentary ascogonia and strains without pro22, pro45, Smpp2Ac1, and sci1 genes only generate unpigmented protoperithecia, whereas pigmented protoperithecia are formed in the Smmob3 deletion mutant. This indicates that SmMOB3 is involved in protoperithecia to perithecium transition (Beier et al., 2016, Bernhards \& Pöggeler, 2011, Bloemendal et al., 2012, Bloemendal et al., 2010, Nordzieke et al., 2015, Pöggeler \& Kück, 2004). Genes encoding the SmSTRIPAK complex components pro11 and Smmob3 were shown to be upregulated during sexual development (Bernhards, 2010, Bernhards \& Pöggeler, 2011).

Consistent with the transcript level reported in quantitative reverse-transcription PCR experiments for pro11 (Bernhards \& Pöggeler, 2011), the transcript level of sci1 was very high at day 3 indicating that sci1 and pro11 might regulate similar developmental stages. The sci1 gene was downregulated at day 4, the time point when pro22 became up-regulated (Bloemendal et al., 2010) and was up-regulated again at day 5, 6 and 7. Interestingly, the Smmob3 transcript level was highest at later developmental stages especially at day 6, which corresponds to the Smmob3 deletion mutant phenotype impaired in later developmental stages (Bernhards \& Pöggeler, 2011).

Transcriptional regulation of sci1, pro11, pro22 and Smmob3 might ensure that SmSTRIPAK genes are induced or suppressed at specific time points of the life cycle to initiate and regulate sexual development. Interestingly, MOB3 proteins are conserved among animals and filamentous fungi, which are multicellular organisms and undergo complex cell differentiation but not in yeasts, which are unicellular organisms (Lisa-Santamaría et al., 2012, Pracheil \& Liu, 2013, Singh et al., 2011). 


\section{Discussion}

Thus, it is tempting to speculate that the SmSTRIPAK complex controls early developmental processes through PRO11 and SCI1 and is involved in later developmental processes through the association with PRO22 and PP2A and in protoperithecium to perithecium transition by SmMOB3. The STRIPAK complex in S. macrospora seems to be very dynamic, regulating several pathways through the association and exchange of interaction partners at given time points of the life cycle. Similarly, the Far complex components interact in a chronological order in S. cerevisiae. The Far3 and Far7 proteins interact with the striatin ortholog Far8. Then, the Far3/7/8 complex connects the SLMAP orthologs Far9/10, which are localized to the ER via their TM domains, to the STRIP1/2 ortholog Far11 (Pracheil \& Liu, 2013). In mammals, mutually exclusive STRIPAK complexes were suggested but no sequential order of interaction has been described to date (Goudreault et al., 2009).

In S. macrospora, the phenotypes of SmSTRIPAK complex deletion mutants, pull-down of SmSTRIPAK proteins and transcript levels of SmSTRIPAK genes suggest a regulation of the SmSTRIPAK complex through its different components during sexual development (Beier et al., 2016, Bernhards \& Pöggeler, 2011, Bloemendal et al., 2012, Bloemendal et al., 2010, Nordzieke et al., 2015). SmSTRIPAK complex components might also interact before the SmSTRIPAK complex is regulated through GCKIII kinases by phosphorylation or through the phosphatase SmPP2A by dephosphorylation events. Alternatively, the SmSTRIPAK complex itself might regulate signaling by phosphorylation/ dephosphorylation of target proteins.

Figure 24 summarizes the importance of the SmSTRIPAK complex, through the interaction of the central protein PRO11 with other SmSTRIPAK complex components, during the life cycle of $S$. macrospora. The figure does not exclude several proteins to interact at the same time but shows necessity of the SmSTRIPAK components during the life cycle of S. macrospora.

Unfortunately, using conventional RNA isolation, it is not possible to investigate transcript levels of very young, one and two days old mycelium of $S$. macrospora because sufficient amounts of mycelium for RNA isolation are generated from day 3 to 7. To overcome this problem in future, laser microdissection (LM) and RNA-seq might be applied (Teichert et al., 2012). 


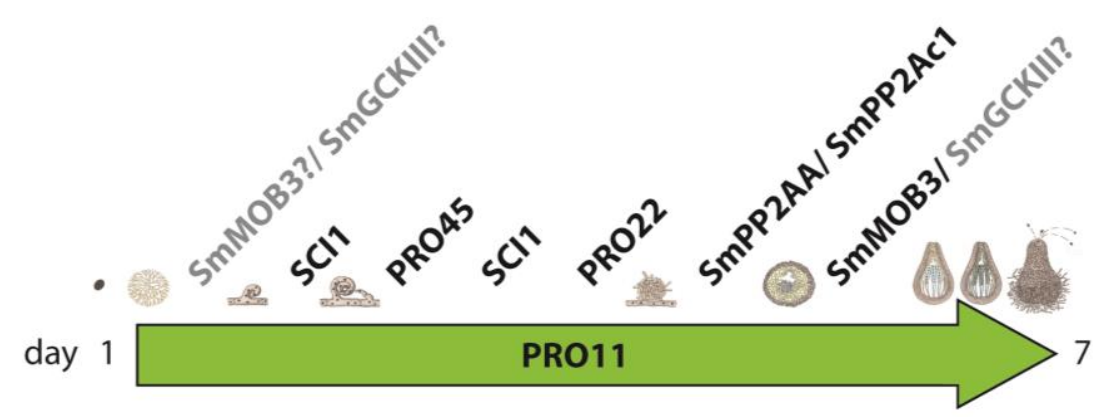

Figure 24: Speculative representation of SmSTRIPAK proteins required during the life cycle of S. macrospora

S. macrospora germinal center III kinase(s) and SmMOB3 might associate with PRO11 during vegetative growth. PRO45 and SCI1 interact with PRO11 in early developmental stages, followed by PRO22. SmPP2AA and SmPP2Ac1 might be required before transition from unpigmented to pigmented protoperithecia. Finally, SmMOB3 and possibly SmGCKIII mediate transition of protoperithecia to mature fruiting bodies. Very early (day 1-2) and late developmental stages (later than day 7) are not investigated and are therefore colored in gray.

\subsection{The central region of SCl1 oligomerizes and interacts with PRO11}

Small CC proteins associate with the STRIPAK in other organisms (Table 1). Consistent with the findings in animals and yeasts, a direct interaction of SCl1 and PRO11 could be shown by $\mathrm{Y} 2 \mathrm{H}$ experiments. The $\mathrm{N}$-terminal region of PRO11, between the predicted $\mathrm{CaM}$-binding site and the WD-repeats, is required for the interaction with SCI1 (Figure 25). Interestingly, the region of PRO11 which is required for interaction with PRO22 overlaps with the region required for interaction with SCI1 (Bloemendal et al., 2012). Thus, SCl1 and PRO22 might compete for the interaction with PRO11 or many SmSTRIPAK complex proteins associate to form a supercomplex in which PRO11 oligomerizes (Bloemendal et al., 2012).

Like Far3, SCl1 oligomerizes via its central region (Lai et al., 2011). Furthermore, the central region of SCl1 spanning AA 63-207 was sufficient to partially complement the sci1 deletion phenotype (Figure 17). Thus, showing that the central region of $\mathrm{SCl1}$, which is also required for interaction with PRO11, is essential for its function in fruiting-body development in S. macrospora (Figure 17). Additionally, the N-terminal region of SCl1 interacts with the SmSTRIPAK complex component PRO45 in $\mathrm{Y} 2 \mathrm{H}$ experiments without the need of the PRO45 TM domain (Figure 16).

However, PRO11 and PRO45 did not directly interact in $\mathrm{Y} 2 \mathrm{H}$ experiments (pers. comm. Steffen Nordzieke), which was confirmed in this study. In sum, SCI1 interacts with the known SmSTRIPAK 


\section{Discussion}

complex components PRO11 and PRO45 in $\mathrm{Y} 2 \mathrm{H}$ experiments (Figure 25) and might mediate or stabilize the interaction of PRO11 and PRO45. However, it remains a task for the future to define which region of PRO45 is required for interaction with SCI1.

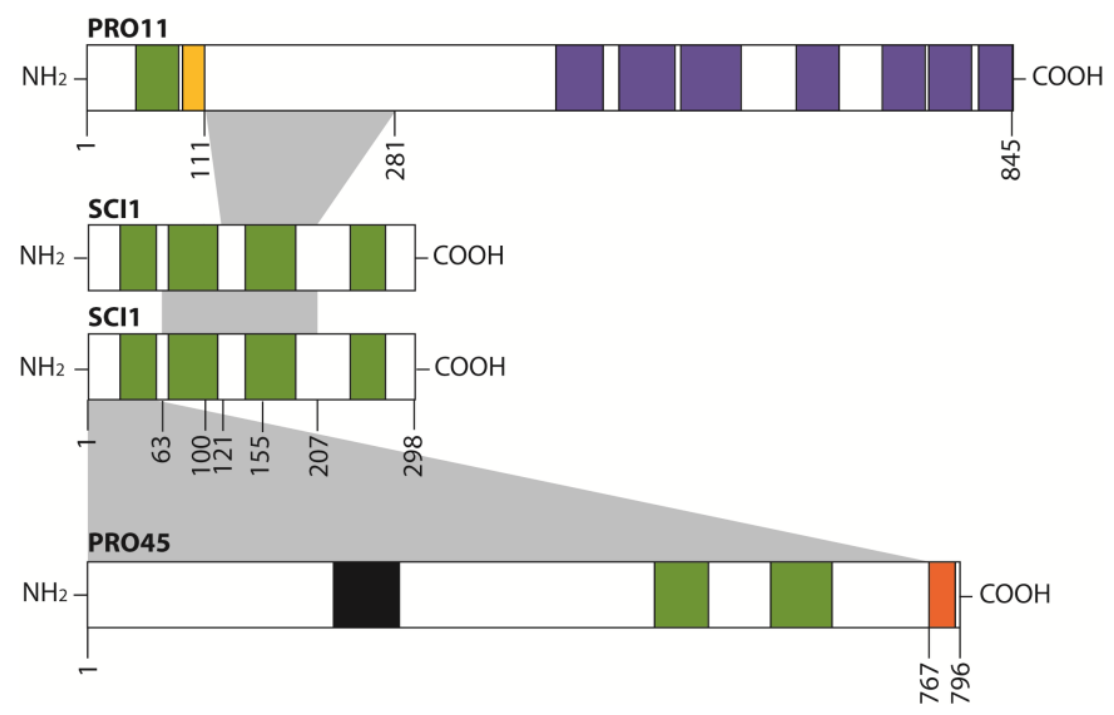

Figure 25: Interactions sites required for SCI1-PRO11, SCI1-SCI1 and SCI1-PRO45 interaction

$\mathrm{Y} 2 \mathrm{H}$ experiments with PRO11 and SCI1 fragments demonstrated that the $\mathrm{N}$-terminal region of PRO11 is required for interaction with the central region of $\mathrm{SCl} 1$. The $\mathrm{N}$-terminal region of $\mathrm{SCl} 1$ is required for interaction with PRO45. Position of AAs is given in numbers. Coiled-coil regions of SCI1, PRO11 and PRO45 are indicated in green. The predicted calmodulin-binding site of PRO11 is indicated in yellow and the WDrepeats are presented in purple. The forkhead-associated domain and the transmembrane domain of PRO45 are presented in black and orange, respectively.

\subsection{Functional conservation of STRIPAK complex-associated proteins containing coiled-coil domains}

Pracheil and Liu (2013) assumed that the CC proteins SIKE/FGFR1OP2, Far3 and Far7 and Csc4 have the same structural role in the STRIPAK complex of different species. Also in S. macrospora, SCI1, a small CC protein, associated with the SmSTRIPAK complex. Although small CC proteins seem to have the same function in the STRIPAK complex of animals and fungi, mammalian FGFR1OP2 or yeast $F A R 3$ and $F A R 7$ could not complement the sterile phenotype of the S. macrospora $\triangle$ sci strain (data not shown).

In $\mathrm{Y} 2 \mathrm{H}$ experiments of this study, the central region of $\mathrm{SCl} 1$ with the second and third $\mathrm{CC}$ domains was shown to be required for interaction with the yeast Far3 protein. Whereas for interaction with Far7 the N-terminal region of SCI1 was required (Figure 19). Consistently, in S. cerevisiae, the Far3 


\section{Discussion}

protein self-interacts through its central region and its C-terminal CC domain and Far3 and Far7 interact with each other without the presence of other Far complex components (Lai et al., 2011, Pracheil \& Liu, 2013).

Yeast strains with the central region of SCl1 but not with the full-length SCl1 grew better in presence of Far3 (Figure 19). The N-terminal and C-terminal region of SCI1 might hamper interaction with Far3 because the protein is bigger than Far3, limiting accessibility of the interaction site for Far3. However, SCl1 did not interact with the yeast striatin ortholog Far8, which might be caused by low sequence similarity of Far8 and PRO11. Far8, in which the WD region is missing might also adopt a different conformation than PRO11 (Goudreault et al., 2009).

SCl1 oligomerization and interaction of $\mathrm{SCl} 1$ with $\mathrm{Far} 3$ and $\mathrm{Far} 7$ via $\mathrm{Y} 2 \mathrm{H}$ indicate a functional conservation for STRIPAK-complex associated small CC proteins, in S. cerevisiae and S. macrospora. Nevertheless, S. macrospora is a multicellular organism undergoing tightly regulated differentiation processes (Pöggeler et al., 2006, Teichert et al., 2014) whereas S. cerevisiae is a unicellular organism. Therefore, STRIPAK-complex regulation or function in S. macrospora is most likely more complex than in baker's yeast and FAR3 and FAR7 cannot compensate for the loss of sci1. Similarly, attempts to complement the sterile $\Delta$ Smmob3 strain with a mouse Mob3 (phocein) construct failed, most likely because of the low level of homology between the mammalian and the fungal Mob3 (phocein) gene (Bernhards \& Pöggeler, 2011).

Protein size and also number of small CC domain containing proteins associated with the STRIPAK complex is different in mammals, yeast and filamentous fungi (Figure 9). Regulation of gene expression, folding of the protein, protein modification and conformation of interaction sites and interaction with STRIPAK complex orthologs might be different between distantly related species.

\subsection{SCI1 is part of the core SmSTRIPAK complex in S. macrospora}

In mammals, the core STRIPAK complex consist of striatin, PP2A with its scaffolding and the catalytic subunit, STRIP1/2, SLMAP, and the CC domain proteins SIKE/FGFR1OP2. GCKIII kinases MST4/STK24/STK25 and the kinase activator MOB3 (phocein) associate with the core STRIPAK complex in animals and filamentous fungi (Goudreault et al., 2009) (Table 1). Similarly, of in

D. melanogaster, the core STRIPAK complex component: Cka (striatin), Pp2A-29B (PP2AA) and Mts (PP2Ac1), the STRIP1/2 ortholog CG11526, the SLMAP ortholog CG17494, GCKIII kinases and the 


\section{Discussion}

kinase activator Mob4 are found (Ashton-Beaucage et al., 2014, Ribeiro et al., 2010). CG17494 (SLMAP) was identified with in silico analysis and is highly expressed during embryonal development but was not shown to interact with other dSTRIPAK complex components (Ribeiro et al., 2010). However, depletion of CG17494 (SLMAP) or other dSTRIPAK components suppresses activation of the dJnk pathway controlling epithelial sheet movement and dorsal closure in Drosophila embryos suggesting that the SLMAP ortholog CG17494 acts together with Cka, Mob4 and the STRIP1/2 ortholog CG11526 (Ashton-Beaucage et al., 2014, Ribeiro et al., 2010). In yeasts, no kinase activator was identified but Far3 and Far7/Csc4, Far8/Csc3 (striatin), Far9/10/Csc1 (SLMAP) and Far11/Csc2 (STRIP1/2) associate in the Far and SIP complex of S. cerevisiae and S. pombe, respectively (Lai et al., 2011, Lisa-Santamaría et al., 2012, Pracheil \& Liu, 2013, Singh et al., 2011).

In S. macrospora, the interaction of PRO11 with SCI1, PRO22 and PRO45 resembles the situation in yeasts but additionally SmMOB3 interacts with PRO11 as described for striatin and MOB3 orthologs in animals (Baillat et al., 2001, Bernhards \& Pöggeler, 2011, Goudreault et al., 2009, Ribeiro et al., 2010) (Figure 26).

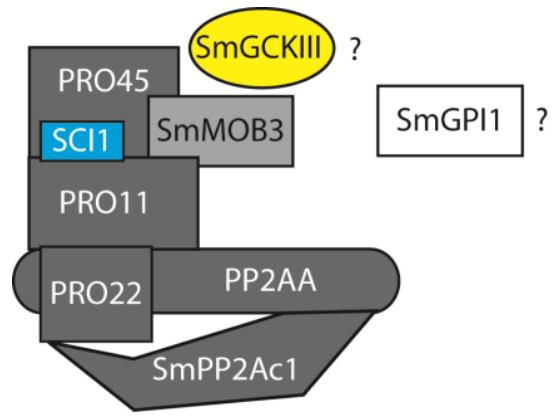

\section{Figure 26: Schematic representation of the STRIPAK complex in S. macrospora}

The striatin ortholog PRO11 acts as scaffold to bring SmSTRIPAK complex proteins together. PRO11 interacts with the kinase activator SmMOB3, the SLMAP ortholog PRO45, the STRIP1/2 ortholog PRO22 and with the small coiled-coil protein SCI1. The phosphatase catalytic subunit (SmPP2Ac1) is attached to the SmSTRIPAK via PP2AA and PRO22. SmGCKIII kinases might counteract the phosphatase activity via SmMOB3. Interactions presented are derived from Co-IP, Y2H and pull-down experiments (Beier et al., 2016, Bernhards \& Pöggeler, 2011, Bloemendal et al., 2012, Bloemendal et al., 2010, Nordzieke et al., 2015).

\subsection{SCl1 and PRO11 colocalize at the nuclear envelope}

For striatins many possible localizations were described up to date. Csc3 in S. pombe associates with early mitotic spindle-pole bodies (SPB) and the Golgi (Frost et al., 2012, Singh et al., 2011). 


\section{Discussion}

Far8 and other Far complex components Far9, Far10, Far11 and the two small CC proteins Far3 and Far7 were shown to localize to the ER in S. cerevisiae (Pracheil \& Liu, 2013). In S. pombe, Csc4 was GFP-tagged but could not be localized so far (Singh et al., 2011). In A. nidulans and F. verticillioides, the striatin orthologs were localized to the nuclear envelope (NE) and the ER (Wang et al., 2010, Zhang et al., 2017). HAM-3 in N. crassa was suggested to localize to the NE and punctiform endomembrane system (Dettmann et al., 2013).

This is the first study, showing that a SmSTRIPAK complex-associated small CC protein colocalizes with the striatin ortholog PRO11 at the NE in S. macrospora (Figure 20 D). Also, signals for PRO11 and $\mathrm{SCl} 1$ fluorescent fusion proteins were detected in the punctiform endomembrane system and filamentous structures sometimes emerging from the nucleus (Figure $20 \mathrm{D}$, Figure $S 12$ ). The nuclear envelope is continuous with the ER (Watson, 1955). Therefore, it is likely that SmSTRIPAK complex proteins associate with the ER, making it difficult to distinguish clearly between NE and ER in fluorescence microscopy because of their close connection. Interestingly, PRO11, SmMOB3 and SCI1 pull-downs coupled to LC/MS identified two putative nucleoporins (SMAC_02409 and SMAC_00774), as potential interaction partner of both proteins indicating that SmSTRIPAK complex components might be directly connected to the NE (Table S 1, Table S 3, Table S 4).

However, the STRIPAK complex might also be involved in ER to Golgi transport. Mammalian striatins contain a caveolin-binding domain to interact with caveolin (Gaillard et al., 2001). Cav1 synthesis takes place in the ER and Cav1 is transported to the Golgi (Hayer et al., 2010). Mammalian SG2NA and zinedin interact with Cav1 (Gaillard et al., 2001) indicating a possible role of the STRIPAK complex in ER to Golgi transport. However, filamentous fungi do not encode caveolin orthologs (Nowrousian, 2010, Pöggeler \& Kück, 2004). Additionally, in mammalian fibroblast cells, SG2NA was shown to maintain ER homeostasis (Jain et al., 2017).

Recently, SLMAP the ortholog PRO45 of S. macrospora was shown to localize to the NE, ER and mitochondria. It was suggested that PRO45 might bridge the NE and the outer mitochondrial membrane for signaling (Nordzieke et al., 2015). However, no alteration of nuclei or mitochondria was observed in the $\Delta$ sci1 strain (Figure S 13). Similarly, the SmSTRIPAK complex of S. macrospora might associate to the NE before it is transported to the ER. 


\subsection{SCl1 might be involved in vesicular transport}

Current and previous studies demonstrate that $\Delta$ sci $1, \Delta$ pro11 and $\Delta$ Smpp2ac1 mutants have a strongly reduced vegetative growth rate (Beier et al., 2016, Bernhards, 2010), which might be caused by impaired transport of endosomes (Peñalva, 2010, Shaw et al., 2011). In silico analysis of SmMOB3 indicates a role in vesicular transport (Bernhards \& Pöggeler, 2011) and proteins involved in vesicular transport were identified in PRO11 and SmMOB3 pull-downs coupled to LC/MS (Table S 1, Table S 2, Table S 3).

Therefore, endosomal transport of early endosomes (EEs) in the wt and the $\Delta$ scil strain was investigated using the N-terminally eGFP-tagged Rab5 protein from the ascomycete $Z$. tritici (Kilaru

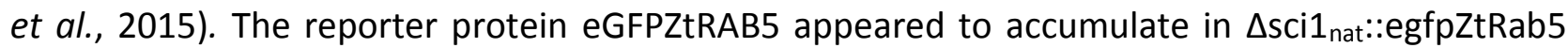
hyphae compared to wt::egfpZtRab5. However, EE transport was not disturbed because in $\Delta$ sci1 nat::egfpZtRab5 EEs moved along filamentous structures through the hyphae (Video 8). The eGFP protein is very stable and was detected in vacuoles of wt::egfpZtRab5 indicating that the eGFPZtRab5 protein is degraded. However, the lumen of vacuoles in $\Delta$ sci1 $_{\text {nat }}:$ egfpZtRab5 hyphae was not stained by eGFP (Figure $22 \mathrm{~B}$ ).

In S. cerevisiae, the SLMAP ortholog Far9 directs sorting of proteins to vacuoles in regulating vesicular traffic. Deletion of yeast FAR9 (SLMAP) results in aberrant vacuoles and strongly decreased secretion of the $\alpha$-factor (Bonangelino et al., 2002). In the study of Bonangelino et al. (2002), the deletion mutant Far9 showed a strong aberrant vacuolar phenotype and the Far 11 deletion mutant (STRIP1/2) showed a weakly aberrant vacuolar phenotype (Bonangelino et al., 2002).

Thus, the lack of free eGFP in $\Delta$ sci1 $_{\text {nat }}:$ :egfpZtRab5 vacuoles might be caused by a vesicle transport defect, nevertheless, movement of EEs was observed in $\Delta$ sci1 nat::egfpZtRab5 (Video 8). Additionally, FM4-64 was internalized and stained vacuolar membranes in the wt and in the sci1 deletion background strains arguing against a complete vesicle to vacuole transport defect in the sci1 deletion strain (Fischer-Parton et al., 2000, Hoffmann \& Mendgen, 1998, Read et al., 1998) (Figure S 15 B). 


\subsubsection{The STRIPAK complex has a role in ER to Golgi transport}

In mammals, the STRIPAK complex was connected to ER to Golgi transport (Kean et al., 2011) and striatin, SG2NA, STRIP1/2 and MOB3 localize to the Golgi (Frost et al., 2012). Frost and colleagues (2012) speculated that the STRIPAK complex might be involved in Golgi fragmentation and targeting of Golgi fragments to centrosomes during mitosis.

The Golgi apparatus in mammals is partitioned between dividing cells but has to be fragmented before. The Golgi ribbon is unstacked in G2 phase, which is mediated by several kinases (Acharya et al., 1998, Colanzi et al., 2000, Colanzi et al., 2003, López-Sánchez et al., 2009, Ruan et al., 2004, Shorter \& Warren, 2002, Sütterlin et al., 2001). Subsequently, the Golgi proteins GM130 and GRASP65 are phosphorylated and the cell enters mitosis (Hidalgo Carcedo et al., 2004, Sütterlin et al., 2002) (Figure $27 \mathrm{~A}$ ).

A

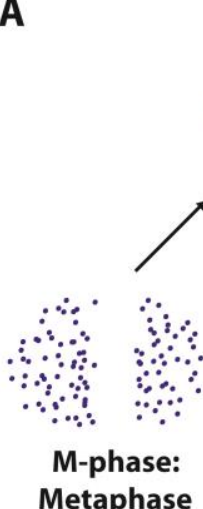

S-phase

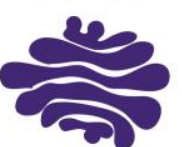$$
\text { . }
$$$$
\leftarrow
$$$$
\text { se }
$$

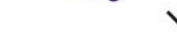

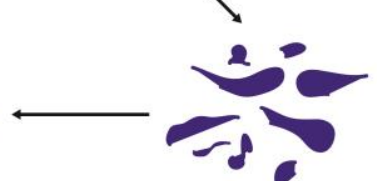

G2/ M-phase: Prophase
B

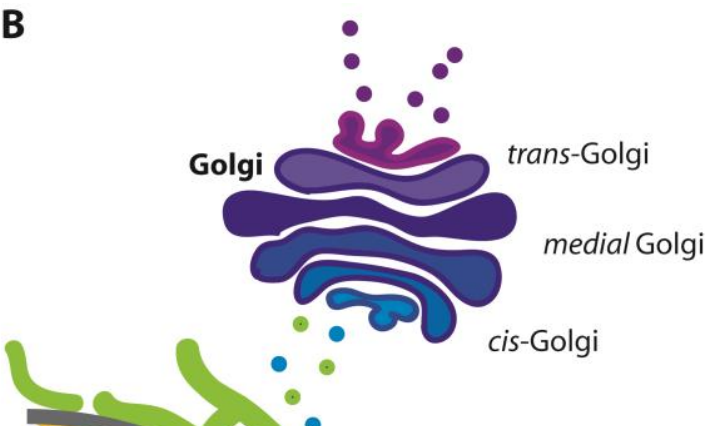

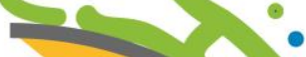
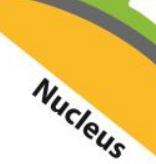

.
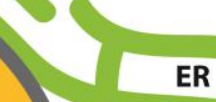

ER
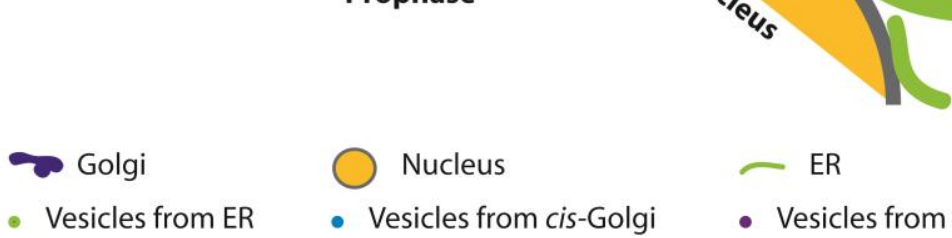

Nucleus
- Vesicles from cis-Golgi

- Vesicles from ER

$$
\text { - Vesicles from trans-Golgi }
$$

Figure 27: The Golgi apparatus, vesicle transport and the Golgi checkpoint in mammals

A: Golgi in S-phase appears as ribbons and is fragmented into Golgi stacks before the cell enters Mitosis. Golgi stacks are further fragmented and partitioned between daughter cells. B: Schematic representation of ER-Golgi forward- and back-transport of cargoes. ER derived vesicles are transported to the cis-Golgi, vesicles from the cis-Golgi can be targeted to the ER or vesicles are secreted trough the trans-Golgi for transport to defined cell sites. Modified according to De Matteis and Luini (2008) and Corda et al. (2012). 
In mammals, Golgi stacks are further fragmented appearing as a "haze" to be partitioned between the daughter cells (Altan-Bonnet et al., 2004, Colanzi \& Corda, 2007, Shorter \& Warren, 2002). After partitioning between daughter cells, Golgi fragments are re-stacked to Golgi ribbons via dephosphorylation of the Golgi stacking protein GRASP65 by PP2A, a phosphatase, which is also present in the STRIPAK complex (Tang et al., 2008, Goudreault et al., 2009) (Figure 27 A) .

The Golgi apparatus of mammals mediates and controls sorting of cargo (Figure 27 B). Proteins synthesized in the ER pass through the Golgi and are transported to their destinations or endosomes are transported back to the Golgi (De Matteis \& Luini, 2008). Final post-translational modification of proteins takes place in the Golgi and lipids are transferred from the ER to the Golgi (De Matteis \& Luini, 2008, Ladinsky et al., 1999, Mogelsvang et al., 2004) (Figure 27 B).

Recently, the SmSTRIPAK component SmMOB3 of S. macrospora was described to interact with the glycosylphosphatidylinositol-anchored protein 1 (SmGPI1) in Y2H experiments (Frey et al., 2015a). GPIs are synthesized in the ER, pass through the Golgi and are anchored in the plasma membrane (Orlean \& Menon, 2007).

In yeasts, the prevacuolar compartment (PVC) mediates protein sorting between the Golgi and vacuoles in an anterograde and retrograde manner. In S. cerevisiae, the vacuolar protein sortingassociated protein 27 (Vps27) associates with vesicles and mediates sorting of vesicles to the vacuoles but also ensures return to the Golgi (Piper et al., 1995). The yeast ortholog Vps21 of mammalian Rab5 is required for Golgi exit into the PVC and mutants lacking Vps21 accumulate vacuolar proteins in the PVC hence vacuolar proteins are not delivered to vacuoles (Gerrard et al., 2000).

This study gives first hints, that the SmSTRIPAK complex might regulate vesicular traffic maybe through the Golgi in S. macrospora. In the scil deletion background, accumulation of eGFPZtRab5eGFP was detected in big punctiform structures in young hyphae and no free eGFP was detected in vacuoles (Figure 22) suggesting a defect in vesicle to vacuole transport.

In mammals, striatins and CCM3 were shown to regulate MST4 association with Golgi in an opposite manner (Kean et al., 2011). Depletion of striatin might block ER to Golgi transport and depletion of CCM3 might prevent secretion from the Golgi. Taken together, the STRIPAK complex in mammals was connected to ER to Golgi transport and vesicle transport from the Golgi, but up to now no $\mathrm{CCM} 3$ ortholog was described in filamentous fungi. 


\section{Discussion}

Deletion of sci1 results in trapping of eGFPZtRab5 in either, the intermediate compartment between ER and Golgi or in the Golgi indicates a possible role of $\mathrm{SCl} 1$ as golgin. Golgins are predominantly CC proteins attached to the Golgi via their C-terminal transmembrane domains (TM) or associate via other membrane bound proteins to the Golgi if they lack a TM domain (D'Angelo et al., 2013).

SCI1 contains four predicted CC domains and, like golgins shows no sequence homology (Witkos \& Lowe, 2015) with other small CC proteins associated with STRIPAK complexes in other distant related species. However, no transmembrane domain was predicted for $\mathrm{SCl} 1$.

Investigation of vesicle transport especially between the ER and Golgi should be performed in $S$. macrospora to dissect how and when the SmSTRIPAK complex might be involved in vesicle transport. In mammalian cells, MOB3 (phocein) was described to function in vesicular traffic. MOB3 (phocein) and SG2NA were localized to the Golgi and treatment with brefeldin $\underline{A}$ (BFA) caused dispersion of MOB3 (phocein) and SG2NA to the cytosol (Baillat et al., 2001). In N. crassa BFA was shown to inhibit ER to Golgi transport of the plasma membrane $\mathrm{H}^{+}$-translocating ATPase (PMA-1) and caused accumulation of PMA-1 in subapical and distal hyphal regions (Fajardo-Somera et al., 2013).

Thus, S. macrospora strains synthesizing SmSTRIPAK complex proteins fused to fluorophores should be investigated upon treatment with BFA to clarify if the SmSTRIPAK complex is involved in ER to Golgi transport. Looking at vesicular transport boarders between the specific compartments might be blurred, because of the continuous exchange and recycling of molecules and membranes within cells. Nothing is known about Golgi morphology in SmSTRIPAK complex deletion mutants of filamentous fungi. Therefore, using reasonable marker proteins might help to clarify at which point the SmSTRIPAK complex might mediate vesicular transport.

\subsubsection{The STRIPAK complex is connected to microtubules and Golgi inheritance}

In this study, a putative dynein ortholog was enriched in SCI1 pull-downs (Figure 18, Table S 4). Also, in PRO11 pull-downs a putative kinesin ortholog and a putative centractin ortholog were identified (Table S 1). Additionally, in PRO11 and SmMOB3 pull-downs a putative myosin-5 ortholog and a putative nucleoporin were found (Table S 3). These data indicate that the S. macrospora STRIPAK complex might be connected to vesicle transport and microtubules. Also in this study, SCI1 


\section{Discussion}

and PRO11 were colocalized to the NE. In previous studies, PRO22 was shown to localize to tubular vacuoles, which are connected to long distance transport along microtubules in hyphae of filamentous fungi (Bloemendal et al., 2010, Darrah et al., 2006, Hyde et al., 1999).

Recently, the S. macrospora STRIPAK complex component PRO45eGFP was localized in the $\Delta$ pro11 background to defined spots in direct proximity of the nucleus, which were suggested to be SPBs (Nordzieke, 2014). Orthologs of STRIPAK complex components in other organisms were also connected to spindle-pole bodies (SPBs). In contrast to the baker's yeast, S. pombe proteins of the septation initiation network (SIN), which is involved in cytokinesis and septation, localize to SPBS (Singh et al., 2011). In N. crassa, the kinases STK-3 and STK-24 but no other STRIPAK complex components were localize to the SPBs in vegetative hyphae (Heilig et al., 2014, Heilig et al., 2013). In mammals, microtubule minus ends are located at centrosomes and it was shown that the dynactin/dynein complex is required for transport of Golgi fragments along microtubules (Burkhardt et al., 1997, Corthésy-Theulaz et al., 1992). Close positioning of the Golgi to the nucleus was suggested to coordinate polarization, mitosis and apoptosis (Hicks \& Machamer, 2005).

The results of this thesis show that, in $\Delta$ sci1::egfpZtrab5, large punctiform structures visualized by the EE marker dispensed like clouds around dark regions, most like representing nuclei before punctiform structures reformed (Video 9). However, the Golgi in filamentous fungi does not exist as stacks but as dispersed cisternae (Breakspear et al., 2007, Hubbard \& Kaminskyj, 2008, Lee \& Shaw, 2008, Suda \& Nakano, 2012). If Golgi cisternae in S. macrospora can be fragmented and how Golgi inheritance is mediated in S. macrospora remains to be determined.

In mammalian cells, the GCKIII kinase STK25 and the GCKII MST1 were found to localize via the cisGolgi protein GM130 to the Golgi and interact with the STRIPAK complex component CCM3 (Preisinger et al., 2004). CCM3 and the GCK kinases are required for Golgi assembly, positioning and cell migration (Fidalgo et al., 2010). The STRIPAK complex components SG2NA, STRIP1 and MOB3 localize to the Golgi and SLMAP is localized to the outer NE or during mitosis to centrosomes and surrounds the mitotic spindle (Baillat et al., 2001, Moreno et al., 2001). MOB3 depletion results in spindle failure (Frost et al., 2012). Thus, it was suggested that SLMAP connects the Golgi, the NE and centrosomes and might be required for Golgi partitioning between daughter cells (Frost et al., 2012, Guzzo et al., 2005, Guzzo et al., 2004a). In D. melanogaster the Mob4 transcript is highly expressed during development of Drosophila embryos and enriched in their nervous system. 
Interestingly, Mob4 defects result in abnormal synaptic development, disorganized microtubules and disrupted axonal transport (Schulte et al., 2010).

In S. macrospora, the SLMAP ortholog PRO45 was shown to directly interact with PRO11 and SmMOB3 and was localized to the NE, the ER and mitochondria. Also, PRO45 was localized to SPBs in the $\Delta$ pro11 background (Nordzieke et al., 2015) (Figure 28). Thus, it is possible that the interaction between PRO45 and the other core SmSTRIPAK complex components in vegetative hyphae is primarily required to mediate vesicle transfer between the ER, Golgi and microtubules. However, if the SmSTRIPAK complex is involved in Golgi inheritance remains to be investigated.

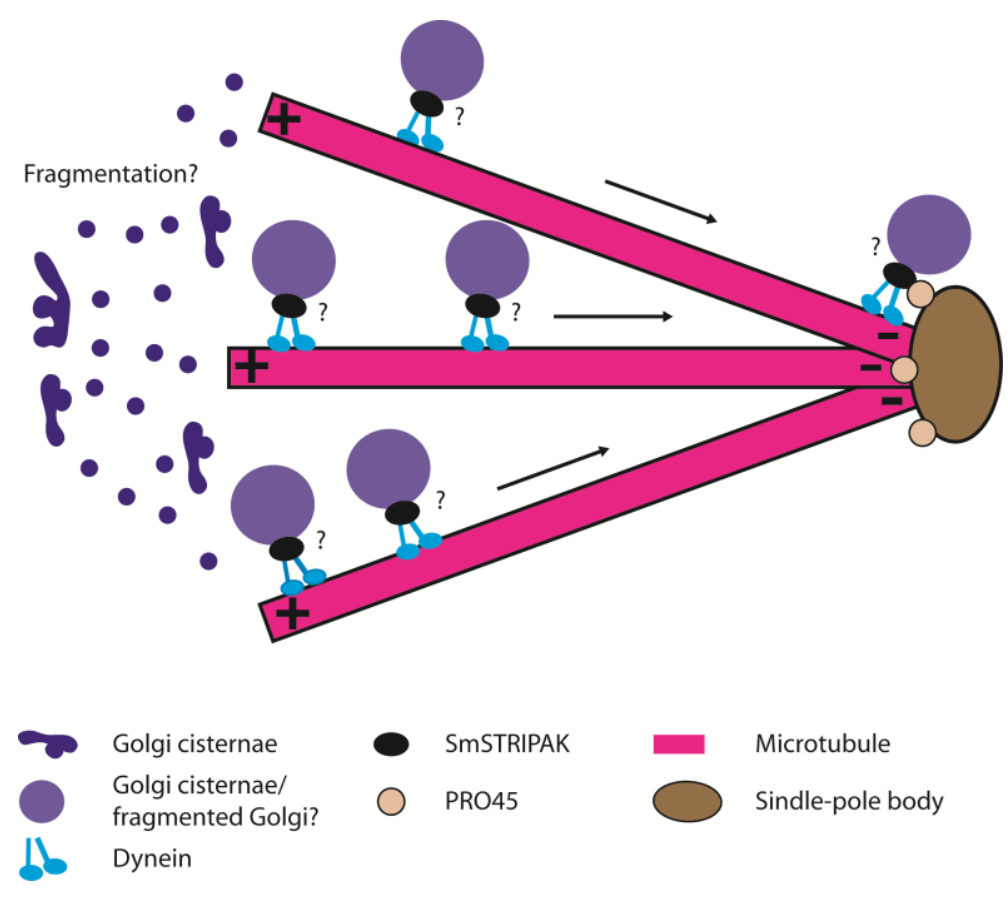

Figure 28: The SmSTRIPAK complex might be involved in dynein-mediate transport

Speculative model of Golgi transport along microtubules in S. macrospora. Dynein heavy chain was enriched in SCl1eGFP pull-downs coupled to LC/MS and SCI1 and PRO45 directly interact in $\mathrm{Y} 2 \mathrm{H}$ experiments. In the S. macrospora $\Delta$ pro11 strain, eGFP-tagged PRO45 localized to the spindle-pole bodies. The SmSTRIPAK complex might be required to target the Golgi to the NE or for Golgi inheritance during mitosis. However, it is not known if Golgi cisternae can be fragmented in S. macrospora.

\subsubsection{The SmSTRIPAK complex mediates septation in ascogonia}

In S. pombe, the SIP complex, which is the ortholog of the STRIPAK complex, negatively regulates SIN to ensure proper septum formation and cytokinesis (Singh et al., 2011). A functional actin ring is required for septum growth and cell-wall synthesis to complete cytokinesis in S. pombe. Absence 
of actin ring allows septum formation but septae are highly disorganized and non-circular (Zhou et al., 2015). The STRIPAK complex-associated protein CCM3 of Caenorhabditis elegans functions in cytokinesis, Golgi stability, localization of cell-surface proteins and is involved in actin organization (Lant et al., 2015, Pal et al., 2017).

In S. macrospora, Smpp2Ac1 and pro22 deletion strains and in the pro22 mutant, no intercalated septae were detected in ascogonia (Beier et al., 2016, Bloemendal et al., 2010). Therefore, a dysregulation of the SIN was suggested in $\Delta$ pro22 and $\Delta$ Smpp2Ac1 strains (Beier et al., 2016). Although PRO22, SmPP2Ac1, PRO11 and SCI1 are SmSTRIPAK complex components intercalating septae were present in ascogonia of $\Delta$ sci1, $\Delta$ pro11 and the pro11 mutant. Also actin polymerization in the vegetative hyphae of the wt and $\Delta$ sci1 background was similar (Figure 21,Video 5, Video 6). However, it cannot be excluded that polymerization of actin in septae of ascogonia might be different from actin polymerization in septae of vegetative hyphae. Therefore, actin polymerization should also be studied in ascogonia of SmSTRIPAK complex deletion mutants in the future.

Septae in fruiting bodies of filamentous fungi show a different morphology than in vegetative hyphae. In Sordaria humana, septae in walls of croziers show intense annular swelling of septal pores. Also many layers of cisternae were shown to be deposited on both sites of the septal pore (Beckett, 1981). In ascogonous hyphae of S. macrospora swollen dense rings were observed at the edge of septal pores. Tubular cisternae were closely associated with the dense rings radiating into the cytoplasm between two cells (Beckett Alan, 1974). However, nothing is known about septalpore ultrastructure in ascogonia of SmSTRIPAK complex deletion mutants. Septal pores of S. macrospora are not closed in vegetative hyphae. However, ascogonous hyphae might be plugged by accumulation of ER and Golgi cisternae to separate two nuclei for karyogamy in one cell. However, such a plug would still allow vesicle transport. A disturbed exchange of vesicles between vegetative hyphae and the protoperithecium or between cells within the developing fruiting body might block perithecium formation because nutrients, enzymes and membrane supply is insufficient or blocked. Also, specialized cells in protoperithecia might be tightly interconnected to ensure nutrition and development of ascospores. It will be very interesting to investigate ER and Golgi ultrastructure in septal pores of SmSTRIPAK complex deletion mutants in comparison to wt septal pores. 


\subsubsection{The STRIPAK complex might be involved in autophagy}

Autophagy takes place in eukaryotes to degrade and recycle cytoplasm for reuse, especially during stress conditions like starvation. The double membrane phagophore sequesters the cargo, expands and elongates and forms the autophagosome. The autophagosome outer membrane fuses with a vacuole and the autophagic body (inner membrane) is released into vacuoles for degradation. Every membrane source in the cell can contribute to autophagosome formation (Feng et al., 2014). In S. cerevisiae, proteins associated with the late and early Golgi were shown to be involved in phagophore extension and thus the generation of the autophagosome. ADP ribosylation factors (Arf) GTPases were considered to be most important for Golgi vesicle formation in the baker's yeast and in mammals (Nie et al., 2003, van der Vaart et al., 2010). However, in mammals formation of the vesicles was not required for autophagosome formation but for maturation of the autophagosome by fusion with EEs (Razi et al., 2009). In this study, accumulation of the EE marker eGFPZtRab5 was detected in the sci1 deletion strain and no eGFP signal was present in large vacuoles indicating a possible defect in autophagy (Figure 22). As the STRIPAK complex in S. macrospora might be connected to the Golgi or ER, it might also be connect to autophagosome formation.

In mammals, Rab5 together with Beclin1 and vacuolar sorting protein 34 (VPS34) regulates autophagosome formation, which is decreased when Rab5 is inhibited (Ao et al., 2014, Ravikumar et al., 2008). Additionally, mammalian TOR kinase activity inhibits activity or expression of Atg proteins (Jung et al., 2010) and Rab5 together with Beclin1 and VPS 34 counteracts mTOR kinase activity to promote autophagosome formation (Simonsen \& Tooze, 2009). Interestingly, in S. cerevisiae the Far complex was connected to TORC1/2 signaling and in S. macrospora proteins of the TORC signaling pathway were pulled-down with SmPP2Ac1 (Baryshnikova et al., 2010, Beier et al., 2016, Lisa-Santamaría et al., 2012, Pracheil et al., 2012). Thus, the SmSTRIPAK complex might regulate autophagosome formation trough TORC signaling. As in S. cerevisiae, SmTORC might be negatively regulated by the SmSTRIPAK. In consequence a non-functional or a lack of SmSTRIPAK complex components could influence autophagosome formation interfering with the autophagy process.

In S. macrospora, fruiting bodies are highly complex structures that arise from two dimensional mycelium and fruiting-body development is coupled to cell death. Deletions of genes encoding 
proteins associated with autophagy show reduced vegetative growth and are sterile, which was also described for strains lacking SmSTRIPAK complex genes (Beier et al., 2016, Bloemendal et al., 2012, Bloemendal et al., 2010, Nordzieke et al., 2015, Pöggeler \& Kück, 2004, Voigt \& Pöggeler, 2013, Werner et al., 2016).

Recently, the striatin ortholog Cka of D. melanogaster was connected to autophagosome transport in neurons via direct interaction of Cka with dynein and Atg8. Depletion of Cka caused accumulation at terminal boutons and depletion of the Hpo kinase decreased autophagic vesicles in terminal boutons. Also, Cka, MOB4 and STRIP were shown to regulate transport of autophagic vesicles and dense-core vesicles (DCV) (Neisch et al., 2017). The authors conclude that Cka interacts with both, the phosphatase and the kinase to regulate axonal transport of DCVs and autophagic vesicles.

In S. macrospora, vesicles containing eGFPZtRab7 are delivered to the vacuoles in both strains wt:egfpZtrab7 and $\Delta$ sci1:egfpZtrab7 because eGFP signals were detected inside vacuoles and at the vacuolar membrane (Figure 23 C, D and E). However, tubular structures in young hyphae of $\Delta$ sci1 appeared shorter and scattered compared to the wt (Figure $23 \mathrm{~A}$ and $\mathrm{B}$ ). Microtubules determine vacuolar morphology and motility (Hyde et al., 1999). Thus, vacuolar transport along microtubules or the connection between vacuoles and microtubules might be disturbed in the $\Delta$ sci1 background compared to wt hyphae. Tubular vacuoles mediate longitudinal transport over millimeters to centimeters within the hyphae (Darrah et al., 2006) and disturbed transport along microtubules in $\Delta$ sci1 might also explain the reduced vegetative growth phenotype.

In mammals, Rab7 is involved in autophagosome to vacuole/lysosome fusion (Gutierrez et al., 2004, Hyttinen et al., 2013, Jäger et al., 2004, Vanlandingham \& Ceresa, 2009). Therefore, it is of great interest to investigate phagophore extension and fusion of autophagosomes with vacuolar membranes in $\Delta$ sci1 compared to wt. Localization of an autophagy marker, for example SmATG8 (Voigt \& Pöggeler, 2013, Werner et al., 2016) and simultaneous localization with eGFPZtRab5 or eGFPZtRab7 in S. macrospora might help to clarify if autophagy is disturbed in SmSTRIPAK complex deletion mutants.

The fusion defect observed in SmSTRIPAK complex deletion mutants might be explained by the inability to reuse molecules and membranes, to normally deliver or transport certain components to the endomembrane system and to specific locations at the membrane. Break down and reuse of cell walls might be reduced and the fusion partner might not be sensed because certain molecules cannot be secreted. Rapid hyphal tip growth requires microtubules (Horio \& Oakley, 


\section{Discussion}

2005) and also endocytic recycling is connected to hyphal growth (Peñalva, 2010, Shaw et al., 2011). However, nothing is known about morphology of microtubules and endocytic recycling in S. macrospora STRIPAK complex mutants and needs to be investigated in the future.

\subsubsection{Vacuolar morphology is altered in $\Delta s c i 1_{\text {nat }}$}

In this study, the LE marker Rab7 from Z. tritici was used to investigate the behavior of LEs in the

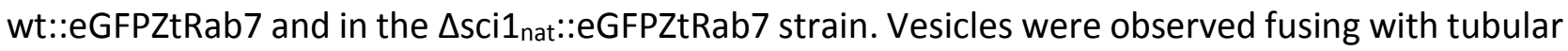
structures followed by spherical vacuoles and vacuoles elongating, splitting and fusing (Figure 23, Video 11). A similar sequence of stained organelles was reported in N. crassa. The vacuolar $\mathrm{Ca}^{2+}$ ATPase NCA-2 and the vacuolar protein VAM-3 were localized to tubular vacuoles in young hyphae, small vesicles and in more distal hyphal regions in large spherical vacuoles (Bowman et al., 2009). However, the tubular structures in the $\Delta$ sci1 background were shorter than in the wt background and vacuoles were also observed in young hyphae. Additionally, large vacuoles without free eGFP

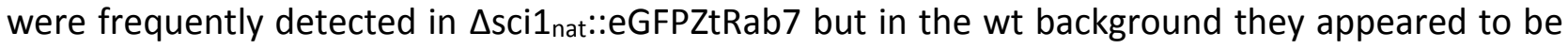
smaller and were only found in very distal hyphal regions (Figure $23 \mathrm{D}$ ). In plants, two types of vacuoles were described. Acidic vacuoles were intensively stained with CMAC whereas storage vacuoles were weakly stained (Swanson et al., 1998). In both strains, wt:egfpZtrab7 and $\Delta$ sci1 $1_{\text {nat }}$ :egfpZtrab7, vacuoles with free eGFP and without eGFP signal as well as intermediates were detected (Figure 23 C, D and E). The LE marker protein eGFPZtRab7 stained the vacuolar membranes of both strains indicating that LE to vacuole fusion is not impaired. Thus, it has to be considered, that the fluorescent protein GFP might be quenched in acidic pH (Bowman \& Bowman, 2010, Enoki et al., 2004). Therefore, roundish structures without an eGFP signal and brightly

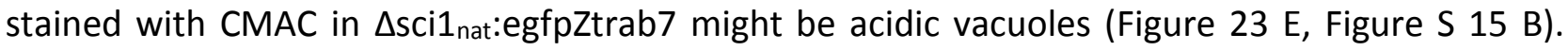
Accumulation of acidic vacuoles in $\Delta s c i 1_{\text {nat }}$ might indicate a defect in reuse of degraded material, more specific in transport of degraded material from vacuoles to a cargo-sorting compartment, hence in secretion. Similar, in S. cerevisiae deletion of yeast FAR9 (SLMAP) results in aberrant vacuoles and strongly decreased secretion of the $\alpha$-factor (Bonangelino et al., 2002).

Vacuoles, the ER, mitochondria and the Golgi are Ca ${ }^{2+}$ reservoirs (Jackson \& Heath, 1993). Calcium level in hyphae of filamentous fungi is highest at the hyphal tip and $\mathrm{Ca}^{2+}$ changes in a pulsatile manner are required for hyphal tip growth (Kim et al., 2012). In N. crassa, the Ca ${ }^{2+}$ pumping ATPase 


\section{Discussion}

NCA-1 localized to the NE, which was considered as specialized region of the ER (Newport \& Forbes, 1987, Prinz et al., 2000, Wedlich-Söldner et al., 2002). Calcium pumping ATPases were also localized to small vesicles and to lysosome-like compartments (Gerasimenko \& Tepikin, 2005). Interestingly, vesicular and vacuolar structures described by investigation of NCAs in N. crassa were similarly visualized in this study with the eGFPZtRab7 reporter construct, and were altered in the sci1 deletion mutant background (Figure 23).

In mammals, striatins interact with calmodulin (CaM) in a $\mathrm{Ca}^{2+}$-dependent manner but it is not known if this is also true for PRO11 in S. macrospora (Castets et al., 1996, Castets et al., 2000, Moreno et al., 2000, Bartoli et al., 1998). However, the C-terminally eGFP-tagged PRO22 protein localizes to tubular vacuoles, a potential $\mathrm{Ca}^{2+}$ reservoir. In contrast to PRO22, the eGFP-tagged HAM-2 was detected at the NE (Dettmann et al., 2013). Also, the striatin ortholog PRO11 localizes to the NE and interacts with PRO22 (Bloemendal et al., 2012, Bloemendal et al., 2010, Gerasimenko \& Tepikin, 2005). Localization of HAPRO11eGFP in the pro11 deletion background and SCI1DsRED in the sci1 deletion background showed that both fusion proteins localize to tubular structures (Figure S $17 \mathrm{~B}$ and C). However, if PRO11 signals in a $\mathrm{Ca}^{2+}$-dependent manner via CaM remains to be investigated.

Localization of eGFPZtRab7 in S. macrospora suggests a highly interconnected long distance transport from young hyphae to old hyphae, maybe also in an anterograde manner. Whether calcium signaling and transport from ER to vacuoles, from vacuoles to ER, or vacuolar transport is mediated by the STRIPAK complex in S. macrospora remains to be determined.

\subsubsection{SCI1 and PRO11 connect the SmSTRIPAK complex to vesicular transport and microtubules}

Microtubules are required for transport of vesicles trough the cytoplasm and dynein or kinesin association mediates the direction (Lu \& Gelfand, 2017). In D. melanogaster, the ortholog of STRIP1/2, Strip regulates dendrite branching and axon elongation of olfactory projection neurons and is suggested to be involved in microtubule organization. The D. melanogaster protein Strip forms a complex with Glued, an ortholog of mammalian p150, at growing ends of microtubules. Therefore, it was suggested that the dSTRIPAK complex together with Glued mediates transport from actin to microtubules (Sakuma et al., 2014). Depletion of the MOB3 ortholog Mob4 was 


\section{Discussion}

connected to abnormal budding events from cellular compartments and a Mob4 mutant shows defects in axonal transport and microtubule organization (Schulte et al., 2010).

In mammals, striatin was connected to dynein (Goudreault et al., 2009). In this study, a putative dynein heavy chain ortholog was identified in SCI1 pull-downs. Furthermore, putative orthologs of proteins associated with vacuolar transport (SMAC_04234 and SMAC_08299), transport from ER to Golgi (SMAC_06397), ER/NE (SMAC_00774) and secretion from trans-Golgi were identified (SMAC_03125) (Figure 18, Figure S 10, Table S 1, Table S 4).

Accumulation of the EE marker eGFPZtRab5 and the more scattered appearance of presumably tubular vacuoles visualized by the LE marker eGFPZtRab7 in young hyphae of $\Delta$ sci1::egfpZtrab5 and $\Delta$ sci1::egfpZtrab7, respectively, indicates vesicle and vacuolar transport defects in the $\Delta$ sci1 background. Taken together, the results of this thesis indicate that SCI1 might be required for long distance transport of vesicles or transfer of vesicles to microtubules, but the cargo could not be identified.

Vesicle transport also involves actin polymerization. Actin was observed at fusion sites of conidial anastomosis tubes of $N$. crassa and it was suggested that actin patches and cables are required for endo- and exocytosis (Berepiki et al., 2010). Secretory vesicles in A. nidulans are loaded with kinesin, dynein and the myosin-5 ortholog MyoE (Pantazopoulou et al., 2014, Peñalva et al., 2017). Transport along actin fibers and actin polymerization should be investigated at the site of fusing hyphae and particularly of fusion between wt and $\Delta$ sci1 hyphae. Especially, because a putative myosin-5 ortholog (SMAC_05008) was identified in PRO11 and SmMOB3 pull-downs, as well as a putative kinesin ortholog (SMAC_04212) and a putative actin-2 protein (SMAC_07827) in PRO11 pull-downs. Also, dynein heavy chain (SMAC_00761) was enriched in SCl1 pull-downs coupled to LC/MS (Figure 18, Table S 1, Table S 2, Table S 3, Table S 4).

Therefore, the STRIPAK complex of filamentous fungi might be directly involved in vesicle transport over long distance as well as in short distance transport requiring actin fibers and myosin to ensure proper material exchange at the fusion sites or between organelles (Figure 29). 


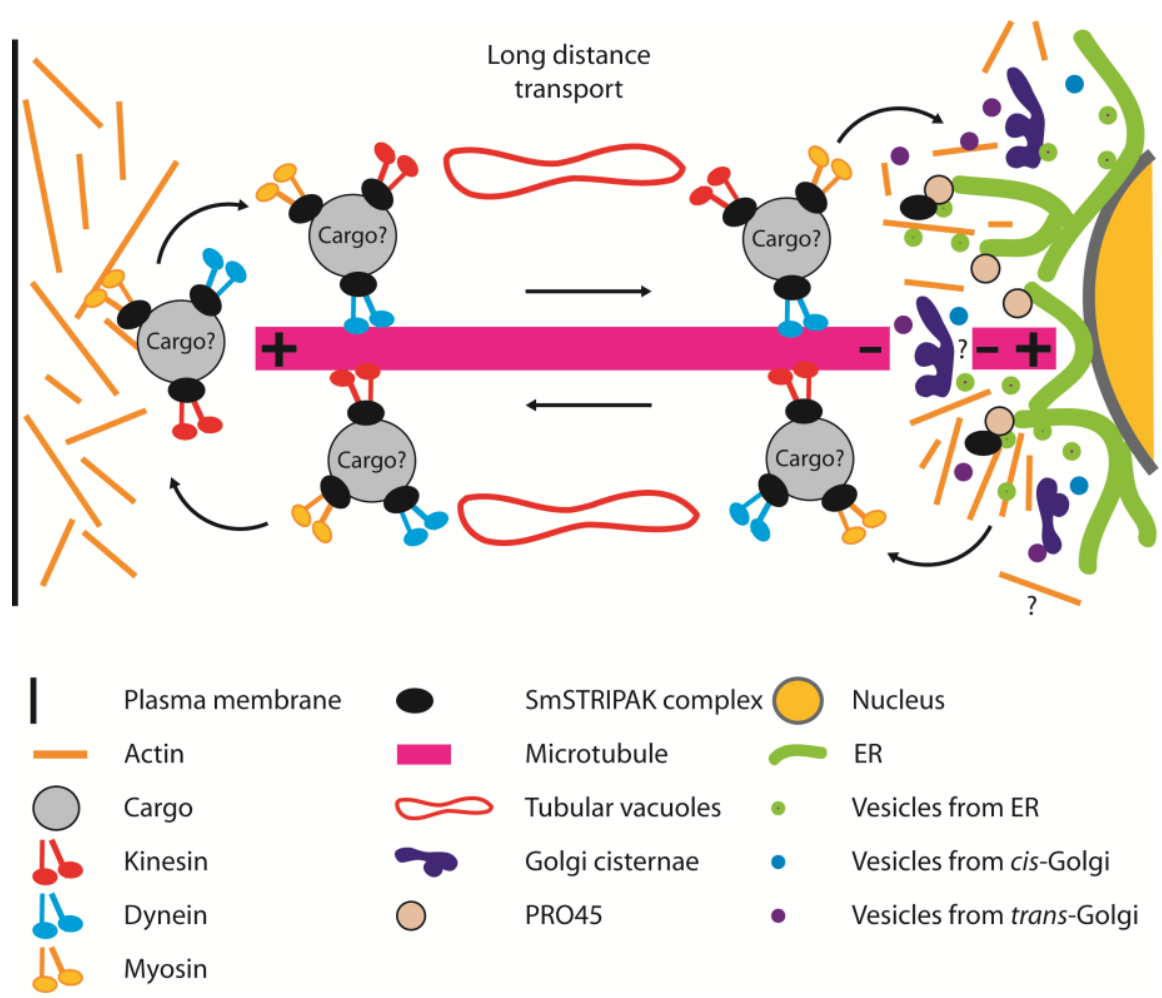

Figure 29: The STRIPAK complex in S. macrospora might be involved in long and short distance transport of vesicles

Speculative representation of the STRIPAK-complex function in S. macrospora. In PRO11, SmMOB3 and SCI1 pull-downs coupled to LC/MS putative orthologs of a kinesin, dynein and a myosin protein were identified. The SmSTRIPAK complex component PRO22 localizes to tubular vacuoles implicated in long distance transport but the SmSTRIPAK can also be located at the nuclear envelope (PRO11, SCI1, SmMOB3 and PRO45). Transport along microtubules between the Golgi and ER as well as possible involvement of actin in short distance transport is suggested but not experimentally proven in S. macrospora.

\subsection{The STRIPAK complex in S. macrospora is dynamic}

Deletion of STRIPAK complex genes in S. macrospora results in strains with similar phenotypes suggesting that the SmSTRIPAK complex signaling requires all known SmSTRIPAK complex components for proper function (Beier et al., 2016, Bernhards \& Pöggeler, 2011, Bloemendal et al., 2012, Bloemendal et al., 2010, Nordzieke et al., 2015). In N. crassa ham-2 (STRIP1/2), ham-3 (striatin) and ppg-1 (PP2Ac) deletion but not $\Delta$ ham-4 (SLMAP) or $\Delta$ mob-3 (MOB3) strains were highly reduced in protoperithecia formation and in hyphal growth (Dettmann et al., 2013, Simonin et al., 2010). It was suggested that HAM-2, HAM-3 and PPG-1 are most important for fruiting-body development and HAM-4 and MOB-3 fulfill accessory tasks (Dettmann et al., 2013). 
In S. macrospora, SCI1, PRO11, PRO22, SmPP2Ac1 and PRO45 seem to function in earlier developmental stages than SmMOB3 (Beier et al., 2016, Bernhards \& Pöggeler, 2011, Bloemendal et al., 2012, Bloemendal et al., 2010, Nordzieke et al., 2015, Pöggeler \& Kück, 2004). Analysis of the scil deletion mutant showed that the phenotype of $\Delta$ sci1 is more similar to the $\Delta$ pro 11 deletion strain phenotype compared to the other SmSTRIPAK complex deletion mutants (Figure 14).

The $\Delta$ Smmob3 strain but not the $\Delta$ pro45 still forms pigmented protoperithecia suggests a slightly different function or more important function in later developmental processes as described in N. crassa (Bernhards \& Pöggeler, 2011, Nordzieke et al., 2015).

STRIPAK complex components were located to many cell sites. In mammals, SLMAP localizes to the NE and centrosomes (Frost et al., 2012), in S. pombe SIP components localize to spindle-pole bodies and Csc2 and Csc3 localize to the NE (Singh et al., 2011). In S. cerevisiae, the Far complex proteins associate with the ER and the Golgi (Lisa-Santamaría et al., 2012, Pracheil \& Liu, 2013) and in D. melanogaster Cka translocates to the nucleus and transports autophagosomes (Chen et al., 2002, Neisch et al., 2017). STRIPAK complex orthologs HAM-2, HAM-3, HAM-4, MOB-3 but not PPG-1 in N. crassa were localized to the NE and punctate endomembrane system (Dettmann et al., 2013). In A. nidulans and F. verticillioides the striatin orthologs StrA and Fsr1 respectively, localize to the NE and the ER (Wang et al., 2010, Zhang et al., 2017).

In S. macrospora, STRIPAK complex components SmMOB3, PRO45, SCI1 and PRO11 localize to the NE whereas PRO22 was localized to tubular vacuoles (Bloemendal et al., 2012, Bloemendal et al., 2010, Nordzieke et al., 2015) (Figure 20, Figure S 12, Figure S 17 B and C). However, tubular structures were also identified with HA and eGFP tagged PRO11, DsRED tagged SCI1 and DsRED tagged SmMOB3 (Figure S 17 B and C). Additional, vacuolar transport in the sci1 deletion background seems to be disturbed but not completely abolished (Figure 22, Video 9).

Loss of pro22 causes accumulation of loosely aggregated hyphae (Bloemendal et al., 2010) instead of a densely packed peridium, which is a thick layer that encloses the developing asci (Lord \& Read, 2011). Hyphal fusion occurs in the hyphae and also in the developing fruiting bodies. Cells in protoperithecia fuse and incorporate melanin when the peridium is formed (Lord \& Read, 2011). In PRO11, SmMOB3 and SCI1 pull-downs coupled to LC/MS identified proteins of the vesicular transport machinery (Figure 8, Figure 18, Table S 1, Table S 2, Table S 3, Table S 4).

In N. crassa, hyphal fusion requires endo- and exocytosis as well as short distance transport of vesicles along actin (Berepiki et al., 2010). Therefore, SCI1 might connect the SmSTRIPAK complex 
to long and short distance transport required for hyphal fusion or stabilizes protein interactions in the SmSTRIPAK complex to ensure transport of yet unidentified cargoes.

The STRIPAK complex is a phosphatase and kinase complex, which suggests that the STRIPAK complex is regulated by phosphorylation events or regulates signaling pathways via phosphorylation or dephosphorylation of specific targets (Goudreault et al., 2009). Deletion of the FHA domain of PRO45 renders S. macrospora sterile and hyphal-fusion defective whereas deletion of the TM domain, which is required for NE localization does not (Nordzieke et al., 2015). In general, the FHA domain recognizes phosphothreonine epitopes on proteins and regulates protein interactions in a phosphorylation-dependent manner (Durocher \& Jackson, 2002). Thus, the SLMAP ortholog PRO45 might be connected to phosphorylation (Nordzieke et al., 2015). PRO45 interacts with PRO11, SmMOB3 and with SCI1 but not with PRO22 and SmPP2Ac1 (Nordzieke et al., 2015). Up to now, PRO45 is the only SmSTRIPAK complex component in S. macrospora, which localizes to SPBs in the $\triangle$ pro11 background indicating a unique function in targeting of SmSTRIPAK complex components to the SPBs, maybe for inheritance of the Golgi. In mammals, SG2NA, STRIP1 and MOB3 localize to the Golgi and depletion of MOB3 results in spindle failure (Baillat et al., 2001, Frost et al., 2012, Moreno et al., 2001). Therefore, in S. macrospora Golgi derived vesicles, decorated with SmSTRIPAK complex components might be targeted by PRO45 to SPBs or to the ER (Figure 30).

Phosphatase activity of SmPP2Ac1 was shown to be required for fruiting-body formation (Beier et al., 2016). The wild type Smpp2ac1 under its endogenous promoter complemented the sterile $\Delta$ Smpp2ac1 phenotype, hyphal fusion and fruiting bodies were detected again. Astonishingly, overexpression of a tap-Smpp2ac1 construct partially complemented the sterile phenotype of Smpp2ac1 but no hyphal fusion was detected. It was concluded that hyphal fusion and fruitingbody development are not necessarily linked (Beier et al., 2016). However, the phosphorylation status of distinct STRIPAK complex components might regulate the activity or localization of the whole complex.

Transition from protoperithecia to perithecia requires intensive cell wall remodeling, cell differentiation and growth (Lord \& Read, 2011, Teichert et al., 2014). The STRIP1/2 ortholog PRO22 in S. macrospora was located to vacuolar tubules and to ascogonia and might be required for long distance transport between protoperithecia and hyphae (Bloemendal et al., 2010). PRO22, PP2AA 
and SmPP2Ac1 interact and might target the SmSTRIPAK complex by dephosphorylation to developing protoperithecia (Figure 30).

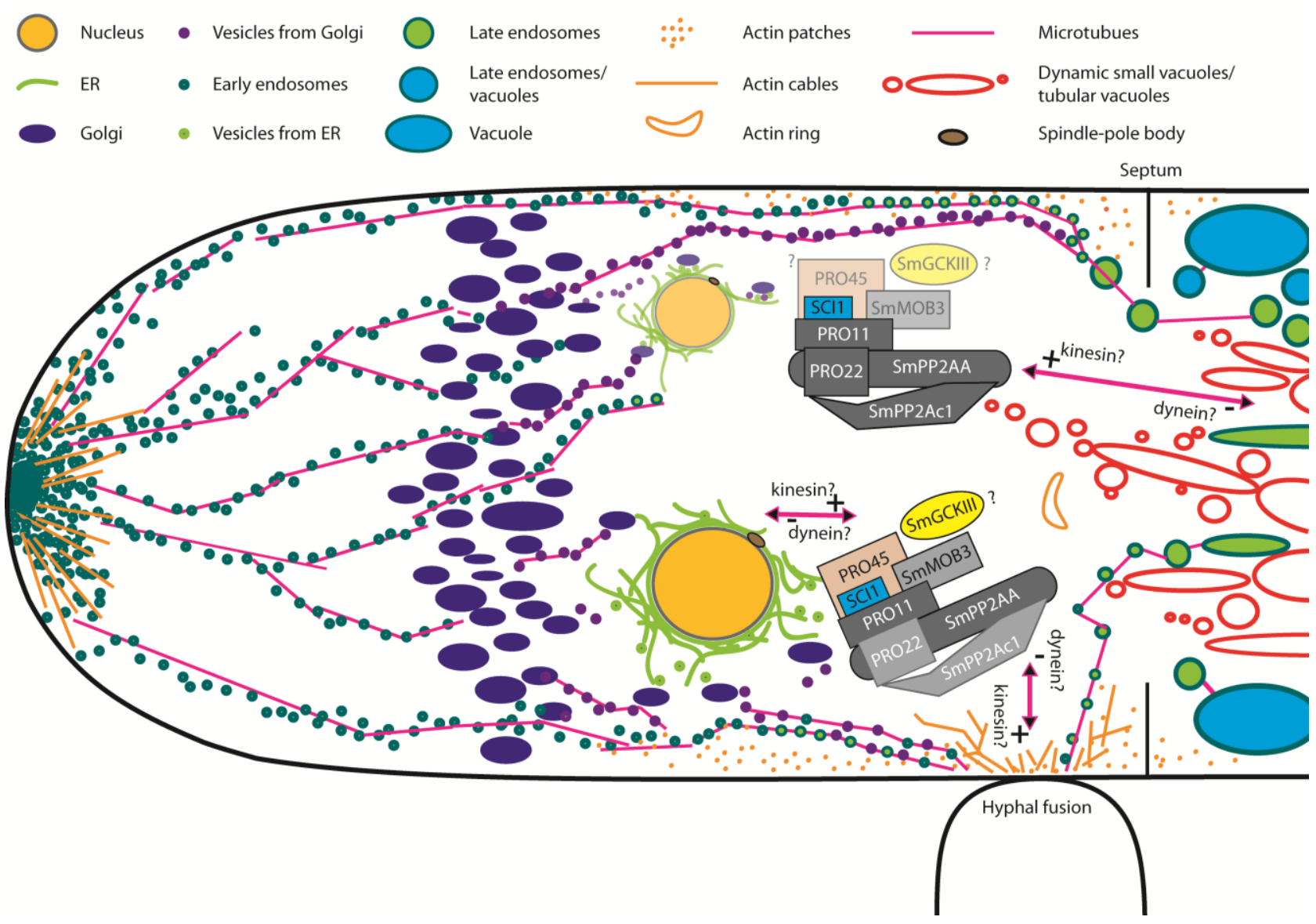

Figure 30: Hypothetical model of a dynamic STRIPAK complex in S. macrospora

The STRIPAK complex might be involved in transport processes in S. macrospora. PRO11, SmMOB3 and SCI1 pull-downs coupled to LC/MS and analysis of endosomal transport with fluorescence microscopy in S. macrospora suggest a direct interaction of the SMSTRIPAK with the transport machinery (short and long distance transport along actin or microtubule, respectively). Phosphatase and kinase activity might regulate SmSTRIPAK complex localization or transport of vesicles targeted by the SmSTRIPAK complex.

Kinases might counteract this mechanism and favor forward transport to vegetative hyphal compartments (Figure 30). Indeed, the STRIPAK complex has a role in vesicular transport in animals. Mammalian MOB3 (phocein) was shown to function in vesicular trafficking and membrane budding (Baillat et al., 2002, Baillat et al., 2001, Haeberlé et al., 2006) and mammalian striatin was connected to dynein (Goudreault et al., 2009). The kinase activator in D. melanogaster Mob4 


\section{Discussion}

regulates synapse formation, axonal transport and microtubule organization (Schulte et al., 2010). Also, in S. macrospora in silico analysis of the kinase activator SmMOB3 suggests a role in vesicular transport (Bernhards \& Pöggeler, 2011).

This thesis and previous studies show that the SmSTRIPAK complex of $S$. macrospora might be directly involved in short and long distance transport of vesicles/vacuoles required for hyphal fusion and fruiting-body development maybe including autophagy processes. Although, mutually exclusively interacting STRIPAK complexes were described in mammals (Goudreault et al., 2009) protein interaction studies, localization of STRIPAK components in S. macrospora, phenotypes of SmSTRIPAK complex deletion mutants and protein pull-down experiments coupled to LC/MS argue for a dynamic SmSTRIPAK complex shuttling through the hyphae to mediate hyphal fusion and sexual development in filamentous fungi. 


\section{References}

Abenza, J.F., Galindo, A., Pinar, M., Pantazopoulou, A., de los Ríos, V. \& Peñalva, M.A., (2012) Endosomal maturation by Rab conversion in Aspergillus nidulans is coupled to dynein-mediated basipetal movement. Molecular Biology of the Cell 23: 1889-1901.

Acharya, U., Mallabiabarrena, A., Acharya, J.K. \& Malhotra, V., (1998) Signaling via mitogen-activated protein kinase kinase (MEK1) is required for Golgi fragmentation during mitosis. Cell 92: 183-192.

Altan-Bonnet, N., Sougrat, R. \& Lippincott-Schwartz, J., (2004) Molecular basis for Golgi maintenance and biogenesis. Current Opinion in Cell Biology 16: 364-372.

Altschul, S.F., Gish, W., Miller, W., Myers, E.W. \& Lipman, D.J., (1990) Basic local alignment search tool. Journal of Molecular Biology 215: 403-410.

Alva, V., Nam, S.Z., Soding, J. \& Lupas, A.N., (2016) The MPI bioinformatics Toolkit as an integrative platform for advanced protein sequence and structure analysis. Nucleic Acids Res 44: W410-415.

Antonny, B., Burd, C., De Camilli, P., Chen, E., Daumke, O., Faelber, K., Ford, M., Frolov, V.A., Frost, A., Hinshaw, J.E., Kirchhausen, T., Kozlov, M.M., Lenz, M., Low, H.H., McMahon, H., Merrifield, C., Pollard, T.D., Robinson, P.J., Roux, A. \& Schmid, S., (2016) Membrane fission by dynamin: what we know and what we need to know. The EMBO Journal 35: 2270-2284.

Ao, X., Zou, L. \& Wu, Y., (2014) Regulation of autophagy by the Rab GTPase network. Cell Death and Differentiation 21: 348-358.

Ashton-Beaucage, D., Udell, C.M., Gendron, P., Sahmi, M., Lefrançois, M., Baril, C., Guenier, A.-S., Duchaine, J., Lamarre, D., Lemieux, S. \& Therrien, M., (2014) A functional screen reveals an extensive layer of transcriptional and splicing control underlying RAS/MAPK signaling in Drosophila. PLOS Biol 12: e1001809.

Baeuerle P. A. \& Henkel, T., (1994) Function and Activation of NF-kappaB in the Immune System. Annual Review of Immunology 12: 141-179.

Bai, S.W., Herrera-Abreu, M.T., Rohn, J.L., Racine, V., Tajadura, V., Suryavanshi, N., Bechtel, S., Wiemann, S., Baum, B. \& Ridley, A.J., (2011) Identification and characterization of a set of conserved and new regulators of cytoskeletal organization, cell morphology and migration. BMC Biology 9: 54.

Baillat, G., Gaillard, S., Castets, F. \& Monneron, A., (2002) Interactions of phocein with nucleosidediphosphate kinase, Eps15, and dynamin I. Journal of Biological Chemistry 277: 18961-18966.

Baillat, G., Moqrich, A., Castets, F., Baude, A., Bailly, Y., Benmerah, A. \& Monneron, A., (2001) Molecular cloning and characterization of phocein, a protein found from the Golgi complex to dendritic spines. Molecular Biology of the Cell 12: 663-673.

Bardin, A.J. \& Amon, A., (2001) MEN and SIN: what's the difference? Nat Rev Mol Cell Biol 2: 815-826.

Bartnicki-Garcia, S., (2002) Molecular biology of fungal development, p. 29-58. Marcel Dekker, New York, NY.

Bartoli, M., Monneron, A. \& Ladant, D., (1998) Interaction of calmodulin with striatin, a WD-repeat protein present in neuronal dendritic spines. Journal of Biological Chemistry 273: 22248-22253.

Bartoli, M., Ternaux, J.P., Forni, C., Portalier, P., Salin, P., Amalric, M. \& Monneron, A., (1999) Downregulation of striatin, a neuronal calmodulin-binding protein, impairs rat locomotor activity. Journal of neurobiology 40: 234-243.

Baryshnikova, A., Costanzo, M., Kim, Y., Ding, H., Koh, J., Toufighi, K., Youn, J.-Y., Ou, J., San Luis, B.-J., Bandyopadhyay, S., Hibbs, M., Hess, D., Gingras, A.-C., Bader, G.D., Troyanskaya, O.G., Brown, G.W., Andrews, B., Boone, C. \& Myers, C.L., (2010) Quantitative analysis of fitness and genetic interactions in yeast on a genome scale. Nat Meth 7: 1017-1024.

Baysal, B.E., Farr, J.E., Goss, J.R., Devlin, B. \& Richard, C.W., 3rd, (1998) Genomic organization and precise physical location of protein phosphatase $2 A$ regulatory subunit $A$ beta isoform gene on chromosome band 11q23. Gene 217: 107-116. 


\section{References}

Becker, D.M. \& Lundblad, V., (2001) Introduction of DNA into yeast cells. Current Protocols in Molecular Biology 13: 13-17.

Beckett, A., (1981) The ultrastructure of septal pores and associated structures in the ascogenous hyphae and asci of Sordaria humana. Protoplasma 107: 127-147.

Beckett Alan, B.H.I., Jordan McLaughlin David (1974) An atlas of fungal ultrastructure. Longman, London.

Beier, A., Teichert, I., Krisp, C., Wolters, D.A. \& Kück, U., (2016) Catalytic subunit 1 of protein phosphatase $2 \mathrm{~A}$ is a subunit of the STRIPAK complex and governs fungal sexual development. mBio 7: e0087000816.

Benmerah, A., Bègue, B., Dautry-Varsat, A. \& Cerf-Bensussan, N., (1996) The ear of adaptin Interacts with the $\mathrm{COOH}$-terminal domain of the Eps15 protein. Journal of Biological Chemistry 271: 12111-12116.

Benoist, M., Baude, A., Tasmadjian, A., Dargent, B., Kessler, J.-P. \& Castets, F., (2008) Distribution of zinedin in the rat brain. Journal of Neurochemistry 106: 969-977.

Benoist, M., Gaillard, S. \& Castets, F., (2006) The striatin family: A new signaling platform in dendritic spines. Journal of Physiology-Paris 99: 146-153.

Berepiki, A., Lichius, A., Shoji, J.-Y., Tilsner, J. \& Read, N.D., (2010) F-actin dynamics in Neurospora crassa. Eukaryotic Cell 9: 547-557.

Bernhards, Y., (2010) Analysis of fruiting-body development of the filamentous fungus Sordaria macrospora. Genetics of Eukaryotic Microorganisms: 1-125.

Bernhards, Y. \& Pöggeler, S., (2011) The phocein homologue SmMOB3 is essential for vegetative cell fusion and sexual development in the filamentous ascomycete Sordaria macrospora. Current Genetics 57: 133-149.

Betz, W., Mao, F. \& Bewick, G., (1992) Activity-dependent fluorescent staining and destaining of living vertebrate motor nerve terminals. The Journal of Neuroscience 12: 363-375.

Betz, W.J., Mao, F. \& Smith, C.B., (1996) Imaging exocytosis and endocytosis. Current Opinion in Neurobiology 6: 365-371.

Birnboim, H.C. \& Doly, J., (1979) A rapid alkaline extraction procedure for screening recombinant plasmid DNA. Nucleic Acids Research 7: 1513-1523.

Bista, P., Zeng, W., Ryan, S., Bailly, V., Browning, J.L. \& Lukashev, M.E., (2010) TRAF3 controls activation of the canonical and alternative NFKB by the lymphotoxin beta receptor. The Journal of Biological Chemistry 285: 12971-12978.

Bloemendal, S., Bernhards, Y., Bartho, K., Dettmann, A., Voigt, O., Teichert, I., Seiler, S., Wolters, D.A., Pöggeler, S. \& Kück, U., (2012) A homologue of the human STRIPAK complex controls sexual development in fungi. Molecular Microbiology 84: 310-323.

Bloemendal, S., Lord, K.M., Rech, C., Hoff, B., Engh, I., Read, N.D. \& Kück, U., (2010) A mutant defective in sexual development produces aseptate ascogonia. Eukaryotic Cell 9: 1856-1866.

Bonangelino, C.J., Chavez, E.M. \& Bonifacino, J.S., (2002) Genomic screen for vacuolar protein sorting genes in Saccharomyces cerevisiae. Molecular Biology of the Cell 13: 2486-2501.

Boscher, C. \& Nabi, I.R., (2012) Caveolin-1: role in cell signaling. Adv Exp Med Biol 729: 29-50.

Bosl, W.J. \& Li, R., Mitotic-exit control as an evolved complex system. Cell 121: 325-333.

Bowman, B.J., Draskovic, M., Freitag, M. \& Bowman, E.J., (2009) Structure and distribution of organelles and cellular location of calcium transporters in Neurospora crassa. Eukaryotic Cell 8: 1845-1855.

Bowman, B.J., Draskovic, M., Schnittker, R.R., El-Mellouki, T., Plamann, M.D., Sánchez-León, E., Riquelme, M. \& Bowman, E.J., (2015) Characterization of a novel prevacuolar compartment in Neurospora crassa. Eukaryotic Cell 14: 1253-1263.

Bowman, E.J. \& Bowman, B.J., (2010) Cellular and molecular biology of filamentous fungi. In: Vacuoles in Filamentous Fungi. J.E. K.A Borkovich (ed). Washington: American Society of Microbiology, 179-190.

Bradford, M.M., (1976) A rapid and sensitive method for the quantitation of microgram quantities of protein utilizing the principle of protein-dye binding. Anal Biochem 72: 248-254.

Brawerman, G., Mendecki, J. \& Lee, S.Y., (1972) A procedure for the isolation of mammalian messenger ribonucleic acid. Biochemistry 11: 637-641. 


\section{References}

Breakspear, A., Langford, K.J., Momany, M. \& Assinder, S.J., (2007) CopA:GFP localizes to putative Golgi equivalents in Aspergillus nidulans. FEMS Microbiology Letters 277: 90-97.

Breitman, M., Zilberberg, A., Caspi, M. \& Rosin-Arbesfeld, R., (2008) The armadillo repeat domain of the APC tumor suppressor protein interacts with striatin family members. Biochimica et Biophysica Acta (BBA) - Molecular Cell Research 1783: 1792-1802.

Burkhardt, J.K., Echeverri, C.J., Nilsson, T. \& Vallee, R.B., (1997) Overexpression of the Dynamitin (p50) subunit of the dynactin complex disrupts dynein-dependent maintenance of membrane organelle distribution. The Journal of Cell Biology 139: 469-484.

Byers, J.T., Guzzo, R.M., Salih, M. \& Tuana, B.S., (2009) Hydrophobic profiles of the tail anchors in SLMAP dictate subcellular targeting. BMC Cell Biology 10: 48.

Campbell, D.H., Sutherland, R.L. \& Daly, R.J., (1999) Signaling pathways and structural domains required for phosphorylation of EMS1/cortactin. Cancer Research 59: 5376-5385.

Carroll, A.M., Sweigard, J.A. \& Valent, B., (1994) Fungal improved vectors for selecting resistance to hygromycin. Genet Newslett 41: 22.

Castets, F., Bartoli, M., Barnier, J.V., Baillat, G., Salin, P., Moqrich, A., Bourgeois, J.P., Denizot, F., Rougon, G., Calothy, G. \& Monneron, A., (1996) A novel calmodulin-binding protein, belonging to the WD-repeat family, is localized in dendrites of a subset of CNS neurons. The Journal of Cell Biology 134: 10511062.

Castets, F., Rakitina, T., Gaillard, S., Moqrich, A., Mattei, M.-G. \& Monneron, A., (2000) Zinedin, SG2NA, and striatin are calmodulin-binding, WD repeat proteins principally expressed in the brain. Journal of Biological Chemistry 275: 19970-19977.

Cenis, J.L., (1992) Rapid extraction of fungal DNA for PCR amplification. Nucleic Acids Research 20: 2380.

Chang, L. \& Gould, K.L., (2000) Sid4p is required to localize components of the septation initiation pathway to the spindle pole body in fission yeast. Proceedings of the National Academy of Sciences of the United States of America 97: 5249-5254.

Chen, C., Shi, Z., Zhang, W., Chen, M., He, F., Zhang, Z., Wang, Y., Miao, H., Feng, M., Wang, W., Zhao, Y., Brown, J.H., Jiao, S. \& Zhou, Z., (2014) Striatins contain a noncanonical coiled coil that binds PP2A A to form a 2:2 heterotetrameric core of striatin-interacting phosphatase and kinase (STRIPAK) complex. Journal of Biological Chemistry 14: 9651-9661.

Chen, H.-W., Marinissen, M.J., Oh, S.-W., Chen, X., Melnick, M., Perrimon, N., Gutkind, J.S. \& Hou, S.X., (2002) CKA, a novel multidomain protein, regulates the JUN N-terminal kinase signal transduction pathway in Drosophila. Molecular and Cellular Biology 22: 1792-1803.

Chen, Y.-K. \& Hsueh, Y.-P., (2012) Cortactin-binding protein 2 modulates the mobility of cortactin and regulates dendritic spine formation and maintenance. The Journal of Neuroscience 32: 1043-1055.

Cheung, J., Petek, E., Nakabayashi, K., Tsui, L.-C., Vincent, J.B. \& Scherer, S.W., (2001) Identification of the human cortactin-binding protein-2 gene from the autism candidate region at 7q31. Genomics 78: 711.

Cho, U.S. \& Xu, W., (2007) Crystal structure of a protein phosphatase 2A heterotrimeric holoenzyme. Nature 445: 53-57.

Chow, A., Hao, Y. \& Yang, X., (2010) Molecular characterization of human homologs of yeast MOB1. International Journal of Cancer 126: 2079-2089.

Christianson, D.W. \& Fierke, C.A., (1996) Carbonic anhydrase: Evolution of the zinc binding site by nature and by design. Accounts of Chemical Research 29: 331-339.

Christianson, T.W., Sikorski, R.S., Dante, M., Shero, J.H. \& Hieter, P., (1992) Multifunctional yeast high-copynumber shuttle vectors. Gene 110: 119-122.

Cohen, C. \& Parry, D.A.D., (1990) $\alpha$-Helical coiled coils and bundles: How to design an $\alpha$-helical protein. Proteins: Structure, Function, and Bioinformatics 7: 1-15.

Colanzi, A. \& Corda, D., (2007) Mitosis controls the Golgi and the Golgi controls mitosis. Current Opinion in Cell Biology 19: 386-393. 


\section{References}

Colanzi, A., Deerinck, T.J., Ellisman, M.H. \& Malhotra, V., (2000) A specific activation of the mitogenactivated protein kinase kinase 1 (MEK1) is required for Golgi fragmentation during Mitosis. The Journal of Cell Biology 149: 331-340.

Colanzi, A., Sutterlin, C. \& Malhotra, V., (2003) RAF1-activated MEK1 is found on the Golgi apparatus in late prophase and is required for Golgi complex fragmentation in mitosis. The Journal of Cell Biology 161: 27-32.

Cole, L., Hyde, G.J. \& Ashford, A.E., (1997) Uptake and compartmentalisation of fluorescent probes by Pisolithus tinctorius hyphae: evidence for an anion transport mechanism at the tonoplast but not for fluid-phase endocytosis. Protoplasma 199: 18-29.

Cole, L., Orlovich, D.A. \& Ashford, A.E., (1998) Structure, function, and motility of vacuoles in filamentous fungi. Fungal Genetics and Biology 24: 86-100.

Collins, B.M., McCoy, A.J., Kent, H.M., Evans, P.R. \& Owen, D.J., (2002) Molecular architecture and functional model of the endocytic AP2 complex. Cell 109: 523-535.

Colot, H.V., Park, G., Turner, G.E., Ringelberg, C., Crew, C.M., Litvinkova, L., Weiss, R.L., Borkovich, K.A. \& Dunlap, J.C., (2006) A high-throughput gene knockout procedure for Neurospora reveals functions for multiple transcription factors. Proc Nat Acad Sci USA 103: 10352-10357.

Corda, D., Barretta, M.L., Cervigni, R.I. \& Colanzi, A., (2012) Golgi complex fragmentation in G2/M transition: An organelle-based cell-cycle checkpoint. IUBMB Life 64: 661-670.

Corthésy-Theulaz, I., Pauloin, A. \& Pfeffer, S.R., (1992) Cytoplasmic dynein participates in the centrosomal localization of the Golgi complex. The Journal of Cell Biology 118: 1333-1345.

Coudreuse, D. \& Korswagen, H.C., (2007) The making of Wnt: new insights into Wnt maturation, sorting and secretion. Development 134: 3-12.

Couet, J., Li, S., Okamoto, T., Ikezu, T. \& Lisanti, M.P., (1997) Identification of peptide and protein ligands for the caveolin-scaffolding domain: Implications for the interaction of caveolin with caveolaeassociated proteins Journal of Biological Chemistry 272: 6525-6533.

Couzens, A.L., Knight, J.D.R., Kean, M.J., Teo, G., Weiss, A., Dunham, W.H., Lin, Z.-Y., Bagshaw, R.D., Sicheri, F., Pawson, T., Wrana, J.L., Choi, H. \& Gingras, A.-C., (2013) Protein interaction network of the mammalian hippo pathway reveals mechanisms of kinase-phosphatase interactions. Science Signaling 6: rs15.

Cox, J. \& Mann, M., (2008) MaxQuant enables high peptide identification rates, individualized p.p.b.-range mass accuracies and proteome-wide protein quantification. Nat Biotech 26: 1367-1372.

Cox, J. \& Mann, M., (2012) 1D and 2D annotation enrichment: a statistical method integrating quantitative proteomics with complementary high-throughput data. BMC Bioinformatics 13: S12.

Crick, F.H., (1952) Is alpha-keratin a coiled coil? Nature 170: 882-883.

Cybulski, N. \& Hall, M.N., (2009) TOR complex 2: a signaling pathway of its own. Trends Biochem Sci 34: 620627.

D'Angelo, G., Uemura, T., Chuang, C.-C., Polishchuk, E., Santoro, M., Ohvo-Rekila, H., Sato, T., Di Tullio, G., Varriale, A., D/'Auria, S., Daniele, T., Capuani, F., Johannes, L., Mattjus, P., Monti, M., Pucci, P., Williams, R.L., Burke, J.E., Platt, F.M., Harada, A. \& De Matteis, M.A., (2013) Vesicular and nonvesicular transport feed distinct glycosylation pathways in the Golgi. Nature 501: 116-120.

da Cruz e Silva, O.B. \& Cohen, P.T.W., (1987) A second catalytic subunit of type-2A protein phosphatase from rabbit skeletal muscle. FEBS Letters 226: 176-178.

Dadgostar, H., Doyle, S.E., Shahangian, A., Garcia, D.E. \& Cheng, G., (2003) T3JAM, a novel protein that specifically interacts with TRAF3 and promotes the activation of JNK 1. FEBS Letters 553: 403-407.

Dan, I., Watanabe, N.M., Kobayashi, T., Yamashita-Suzuki, K., Fukagaya, Y., Kajikawa, E., Kimura, W.K., Nakashima, T.M., Matsumoto, K., Ninomiya-Tsuji, J. \& Kusumi, A., (2000) Molecular cloning of MINK, a novel member of mammalian GCK family kinases, which is up-regulated during postnatal mouse cerebral development. FEBS Letters 469: 19-23. 


\section{References}

Darrah, P.R., Tlalka, M., Ashford, A., Watkinson, S.C. \& Fricker, M.D., (2006) The vacuole system is a significant intracellular pathway for longitudinal solute transport in basidiomycete fungi. Eukaryotic Cell 5: 1111-1125.

De Matteis, M.A. \& Luini, A., (2008) Exiting the Golgi complex. Nat Rev Mol Cell Biol 9: 273-284.

Delpire, E., (2009) The mammalian family of sterile 20p-like protein kinases. Pflügers Archiv - European Journal of Physiology 458: 953-967.

Deng, K.-Q., Wang, A., Ji, Y.-X., Zhang, X.-J., Fang, J., Zhang, Y., Zhang, P., Jiang, X., Gao, L., Zhu, X.-Y., Zhao, Y., Gao, L., Yang, Q., Zhu, X.-H., Wei, X., Pu, J. \& Li, H., (2016) Suppressor of IKKE is an essential negative regulator of pathological cardiac hypertrophy. Nature Communications 7: 11432.

Dettmann, A., Heilig, Y., Ludwig, S., Schmitt, K., Illgen, J., Fleißner, A., Valerius, O. \& Seiler, S., (2013) HAM-2 and HAM-3 are central for the assembly of the Neurospora STRIPAK complex at the nuclear envelope and regulate nuclear accumulation of the MAP kinase MAK-1 in a MAK-2-dependent manner. Molecular Microbiology 90: 796-812.

Donaldson, J.G., Finazzi, D. \& Klausner, R.D., (1992) Brefeldin A inhibits Golgi membrane-catalysed exchange of guanine nucleotide onto ARF protein. Nature 360: 350-352.

Durocher, D. \& Jackson, S.P., (2002) The FHA domain. FEBS Letters 513: 58-66.

Elion, E.A., (2001) The Ste5p scaffold. Journal of Cell Science 114: 3967-3978.

Elleuche, S. \& Pöggeler, S., (2008) Visualization of peroxisomes via SKL-tagged DsRed protein in Sordaria macrospora. Fungal Genet Rep 55: 9-12.

Elleuche, S. \& Pöggeler, S., (2009) $\beta$-Carbonic anhydrases play a role in fruiting body development and ascospore germination in the filamentous fungus Sordaria macrospora. PLoS One 4: e5177.

Engh, I., Nowrousian, M. \& Kück, U., (2007) Regulation of melain biosynthesis via the dihydroxynaphtalene pathway is dependent on sexual development in the ascomycete Sordaria macrospora. FEMS Microbiol Lett 275: 62-70.

Engh, I., Nowrousian, M. \& Kück, U., (2010) Sordaria macrospora, a model organism to study fungal cellular development. European Journal of Cell Biology 89: 864-872.

Enoki, S., Saeki, K., Maki, K. \& Kuwajima, K., (2004) Acid denaturation and refolding of Green Fluorescent Protein. Biochemistry 43: 14238-14248.

Esser, K., (1982) Cryptogams - Cyanobacteria, Algae, Fungi, Lichens. Cambridge University Press, London.

Fajardo-Somera, R.A., Bowman, B. \& Riquelme, M., (2013) The plasma membrane proton pump PMA-1 is incorporated into distal parts of the hyphae independently of the Spitzenkörper in Neurospora crassa. Eukaryotic Cell 12: 1097-1105.

Fankhauser, C., Marks, J., Reymond, A. \& Simanis, V., (1993) The S. pombe Cdc16 gene is required both for maintenance of $\mathrm{p} 34 \mathrm{Cdc} 2$ kinase activity and regulation of septum formation: a link between mitosis and cytokinesis? The EMBO Journal 12: 2697-2704.

Fankhauser, C. \& Simanis, V., (1993) The Schizosaccharomyces pombe Cdc14 gene is required for septum formation and can also inhibit nuclear division. Molecular Biology of the Cell 4: 531-539.

Fankhauser, C. \& Simanis, V., (1994) The Cdc7 protein kinase is a dosage dependent regulator of septum formation in fission yeast. The EMBO Journal 13: 3011-3019.

Farrell, J.A.a.O.F., Patrick H. , (2014) From egg to gastrula: How the cell cycle is remodeled during the Drosophila mid-blastula transition. Annual Review of Genetics 48: 269-294.

Felsenstein, J., (2005) PHYLIP (Phylogeny Inference Package) version 3.6. Department of Genome Sciences, University of Washington, Seattle.

Feng, Y., He, D., Yao, Z. \& Klionsky, D.J., (2014) The machinery of macroautophagy. Cell Research 24: 24-41.

Ferguson, S.M. \& De Camilli, P., (2012) Dynamin, a membrane-remodelling GTPase. Nat Rev Mol Cell Biol 13: 75-88.

Fidalgo, M., Fraile, M., Pires, A., Force, T., Pombo, C. \& Zalvide, J., (2010) CCM3/PDCD10 stabilizes GCKIII proteins to promote Golgi assembly and cell orientation. Journal of Cell Science 123: 1274-1284.

Fields, S. \& Song, O., (1989) A novel genetic system to detect protein-protein interactions. Nature 340: 245246. 


\section{References}

Fischer-Parton, S., Parton, R.M., Hickey, P.C., Dijksterhuis, J., Atkinson, H.A. \& Read, N.D., (2000) Confocal microscopy of FM4-64 as a tool for analysing endocytosis and vesicle trafficking in living fungal hyphae. Journal of Microscopy 198: 246-259.

Fong, H.K., Hurley, J.B., Hopkins, R.S., Miake-Lye, R., Johnson, M.S., Doolittle, R.F. \& Simon, M.I., (1986) Repetitive segmental structure of the transducin beta subunit: homology with the CDC4 gene and identification of related mRNAs. Proceedings of the National Academy of Sciences of the United States of America 83: 2162-2166.

Frey, S., Lahmann, Y., Hartmann, T., Seiler, S. \& Pöggeler, S., (2015a) Deletion of Smgpi1 encoding a GPIanchored protein suppresses sterility of the STRIPAK mutant $\Delta$ Smmob3 in the filamentous ascomycete Sordaria macrospora. Molecular Microbiology 97: 676-697.

Frey, S., Reschka, E.J. \& Pöggeler, S., (2015b) Germinal center kinases SmKIN3 and SmKIN24 are associated with the Sordaria macrospora striatin-interacting phosphatase and kinase (STRIPAK) complex. PLoS One 10: e0139163.

Friedman, A. \& Perrimon, N., (2006) A functional RNAi screen for regulators of receptor tyrosine kinase and ERK signalling. Nature 444: 230-234.

Frost, A., Elgort, Marc G., Brandman, O., Ives, C., Collins, Sean R., Miller-Vedam, L., Weibezahn, J., Hein, Marco Y., Poser, I., Mann, M., Hyman, Anthony A. \& Weissman, Jonathan S., (2012) Functional repurposing revealed by comparing $S$. pombe and S. cerevisiae genetic interactions. Cell 149: 13391352.

Fu, C., Iyer, P., Herkal, A., Abdullah, J., Stout, A. \& Free, S.J., (2011) Identification and characterization of genes required for cell-to-cell fusion in Neurospora crassa. Eukaryotic Cell 10: 1100-1109.

Gaillard, S., Bailly, Y., Benoist, M., Rakitina, T., Kessler, J.-P., Fronzaroli-Molinières, L., Dargent, B. \& Castets, F., (2006) Targeting of proteins of the striatin family to dendritic spines: role of the coiled-coil domain. Traffic 7: 74-84.

Gaillard, S., Bartoli, M., Castets, F. \& Monneron, A., (2001) Striatin, a calmodulin-dependent scaffolding protein, directly binds caveolin-1. FEBS Letters 508: 49-52.

García-Cardeña, G., Martasek, P., Masters, B.S.S., Skidd, P.M., Couet, J., Li, S., Lisanti, M.P. \& Sessa, W.C., (1997) Dissecting the Interaction between nitric oxide synthase (NOS) and caveolin: Functional significance of the NOS caveolin binding domain in vivo Journal of Biological Chemistry 272: 2543725440.

Gerasimenko, O. \& Tepikin, A., (2005) How to measure $\mathrm{Ca}^{2+}$ in cellular organelles? Cell Calcium 38: 201-211.

Gerrard, S.R., Bryant, N.J. \& Stevens, T.H., (2000) VPS21 controls entry of endocytosed and biosynthetic proteins into the yeast prevacuolar compartment. Molecular Biology of the Cell 11: 613-626.

Gietz, R.D. \& Schiestl, R.H., (2007) High-efficiency yeast transformation using the LiAc/SS carrier DNA/PEG method. Nat. Protocols 2: 31-34.

Gonzalez, L., Woolfson, D.N. \& Alber, T., (1996) Buried polar residues and structural specificity in the GCN4 leucine zipper. Nat Struct Mol Biol 3: 1011-1018.

Gordon, J., Hwang, J., Carrier, K.J., Jones, C.A., Kern, Q.L., Moreno, C.S., Karas, R.H. \& Pallas, D.C., (2011) Protein phosphatase 2a (PP2A) binds within the oligomerization domain of striatin and regulates the phosphorylation and activation of the mammalian Ste20-Like kinase Mst3. BMC Biochemistry 12: 54-54.

Goudreault, M., D’Ambrosio, L.M., Kean, M.J., Mullin, M.J., Larsen, B.G., Sanchez, A., Chaudhry, S., Chen, G.I., Sicheri, F., Nesvizhskii, A.I., Aebersold, R., Raught, B. \& Gingras, A.-C., (2009) A PP2A Phosphatase high density interaction network identifies a novel striatin-interacting phosphatase and kinase complex linked to the cerebral cavernous malformation 3 (CCM3) protein. Molecular \& Cellular Proteomics 8: 157-171.

Grallert, A., Boke, E., Hagting, A., Hodgson, B., Connolly, Y., Griffiths, J.R., Smith, D.L., Pines, J. \& Hagan, I.M., (2015) A PP1-PP2A phosphatase relay controls mitotic progression. Nature 517: 94-98. 


\section{References}

Grand, E.K., Grand, F.H., Chase, A.J., Ross, F.M., Corcoran, M.M., Oscier, D.G. \& Cross, N.C.P., (2004) Identification of a novel gene, FGFR1OP2, fused to FGFR1 in 8p11 myeloproliferative syndrome. Genes, Chromosomes and Cancer 40: 78-83.

Green, K.A., Becker, Y., Fitzsimons, H.L. \& Scott, B., (2016) An Epichloë festucae homologue of MOB3, a component of the STRIPAK complex, is required for the establishment of a mutualistic symbiotic interaction with Lolium perenne. Molecular Plant Pathology 17: 1480-1492.

Gruneberg, U., Campbell, K., Simpson, C., Grindlay, J. \& Schiebel, E., (2000) Nud1p links astral microtubule organization and the control of exit from mitosis. The EMBO Journal 19: 6475-6488.

Gu, F., Crump, C.M. \& Thomas, G., (2001) Trans-Golgi network sorting. Cellular and molecular life sciences : CMLS 58: 1067-1084.

Guertin, D.A., Chang, L., Irshad, F., Gould, K.L. \& McCollum, D., (2000) The role of the Sid1p kinase and Cdc14p in regulating the onset of cytokinesis in fission yeast. The EMBO Journal 19: 1803-1815.

Guertin, D.A., Trautmann, S. \& McCollum, D., (2002) Cytokinesis in eukaryotes. Microbiology and Molecular Biology Reviews 66: 155-178.

Gupta, S., Mana-Capelli, S., McLean, J.R., Chen, C.-T., Ray, S., Gould, K.L. \& McCollum, D., (2013) Identification of SIN pathway targets reveals mechanisms of crosstalk between NDR kinase pathways. Current biology 23: 333-338.

Gupta, S. \& McCollum, D., (2011) Crosstalk between NDR kinase pathways coordinates cell cycle dependent actin rearrangements. Cell Division 6: 19.

Gutierrez, M.G., Munafó, D.B., Berón, W. \& Colombo, M.I., (2004) Rab7 is required for the normal progression of the autophagic pathway in mammalian cells. Journal of Cell Science 117: 2687-2697.

Guzzo, R.M., Salih, M., Moore, E.D. \& Tuana, B.S., (2005) Molecular properties of cardiac tail-anchored membrane protein SLMAP are consistent with structural role in arrangement of excitationcontraction coupling apparatus. American Journal of Physiology - Heart and Circulatory Physiology 288: H1810-H1819.

Guzzo, R.M., Sevinc, S., Salih, M. \& Tuana, B.S., (2004a) A novel isoform of sarcolemmal membraneassociated protein (SLMAP) is a component of the microtubule organizing centre. Journal of Cell Science 117: 2271-2281.

Guzzo, Rosa M., Wigle, J., Salih, M., Moore, Edwin D. \& Tuana, Balwant S., (2004b) Regulated expression and temporal induction of the tail-anchored sarcolemmal-membrane-associated protein is critical for myoblast fusion. Biochemical Journal 381: 599-608.

Haan, C. \& Behrmann, I., (2007) A cost effective non-commercial ECL-solution for Western blot detections yielding strong signals and low background. Journal of Immunological Methods 318: 11-19.

Haeberlé, A.-M., Castets, F., Bombarde, G., Baillat, G. \& Bailly, Y., (2006) Immunogold localization of phocein in dendritic spines. The Journal of Comparative Neurology 495: 336-350.

Hanahan, D., Jessee, J. \& Bloom, F.R., (1991) Plasmid transformation of Escherichia coli and other bacteria. Methods Enzymol 204: 63-113.

Hayden, M.S. \& Ghosh, S., (2012) NF-kB, the first quarter-century: remarkable progress and outstanding questions. Genes \& Development 26: 203-234.

Hayden, M.S., West, A.P. \& Ghosh, S., (2006) NF-kB and the immune response. Oncogene 25: 6758-6780.

Hayer, A., Stoeber, M., Bissig, C. \& Helenius, A., (2010) Biogenesis of caveolae: Stepwise assembly of large caveolin and cavin complexes. Traffic 11: 361-382.

Heilig, Y., Dettmann, A., Mouriño-Pérez, R.R., Schmitt, K., Valerius, O. \& Seiler, S., (2014) Proper actin ring formation and septum constriction requires coordinated regulation of SIN and MOR pathways through the germinal centre kinase MST-1. PLOS Genet 10: e1004306.

Heilig, Y., Schmitt, K. \& Seiler, S., (2013) Phospho-regulation of the Neurospora crassa septation initiation network. PLoS One 8: e79464.

Hellman, U., Wernstedt, C., Gonez, J. \& Heldin, C.H., (1995) Improvement of an "in-gel" digestion procedure for the micropreparation of internal protein fragments for amino acid sequencing. Analytical Biochemistry 224: 451-455. 


\section{References}

Hemmings, B.A., Adams-Pearson, C., Maurer, F., Muller, P., Goris, J., Merlevede, W., Hofsteenge, J. \& Stone, S.R., (1990) alpha- and beta-forms of the 65-kDa subunit of protein phosphatase $2 A$ have a similar 39 amino acid repeating structure. Biochemistry 29: 3166-3173.

Hergovich, A., Cornils, H. \& Hemmings, B.A., (2008) Mammalian NDR protein kinases: From regulation to a role in centrosome duplication. Biochimica et Biophysica Acta (BBA) - Proteins and Proteomics 1784: 3-15.

Hergovich, A. \& Hemmings, B.A., (2012) Hippo signalling in the G2/M cell cycle phase: lessons learned from the yeast MEN and SIN pathways. Seminars in cell \& developmental biology 23: 794-802.

Hickey, P.C., Jacobson, D.J., Read, N.D. \& Louise Glass, N., (2002) Live-cell imaging of vegetative hyphal fusion in Neurospora crassa. Fungal Genetics and Biology 37: 109-119.

Hickey, P.C. \& Read, N.D., (2009) Imaging living cells of Aspergillus in vitro. Medical Mycology 47: S110-S119. Hicks, S.W. \& Machamer, C.E., (2005) Golgi structure in stress sensing and apoptosis. Biochimica et Biophysica Acta (BBA) - Molecular Cell Research 1744: 406-414.

Hidalgo Carcedo, C., Bonazzi, M., Spanò, S., Turacchio, G., Colanzi, A., Luini, A. \& Corda, D., (2004) Mitotic Golgi partitioning is driven by the membrane-fissioning protein CtBP3/BARS. Science 305: 93-96.

Hodges, R.S., Sodek, J., Smillie, L.B. \& Jurasek, L., (1973) Tropomyosin: Amino acid sequence and coiled-coil structure. Cold Spring Harbor Symposia on Quantitative Biology 37: 299-310.

Hoeflich, K.P. \& Ikura, M., (2002) Calmodulin in action: Diversity in target recognition and activation mechanisms. Cell 108: 739-742.

Hoffmann, J. \& Mendgen, K., (1998) Endocytosis and membrane turnover in the germ tube of Uromyces fabae. Fungal Genetics and Biology 24: 77-85.

Horio, T. \& Oakley, B.R., (2005) The role of microtubules in rapid hyphal tip growth of Aspergillus nidulans. Molecular Biology of the Cell 16: 918-926.

Horn, T., Sandmann, T., Fischer, B., Axelsson, E., Huber, W. \& Boutros, M., (2011) Mapping of signaling networks through synthetic genetic interaction analysis by RNAi. Nat Meth 8: 341-346.

Hou, M.-C., Salek, J. \& McCollum, D., (2000) Mob1p interacts with the Sid2p kinase and is required for cytokinesis in fission yeast. Current Biology 10: 619-622.

Huang, C., Liu, J., Haudenschild, C.C. \& Zhan, X., (1998) The role of tyrosine phosphorylation of cortactin in the locomotion of endothelial cells. Journal of Biological Chemistry 273: 25770-25776.

Huang, C., Ni, Y., Wang, T., Gao, Y., Haudenschild, C.C. \& Zhan, X., (1997) Down-regulation of the filamentous actin cross-linking activity of cortactin by Src-mediated tyrosine phosphorylation. Journal of Biological Chemistry 272: 13911-13915.

Huang, J., Liu, T., Xu, L.-G., Chen, D., Zhai, Z. \& Shu, H.-B., (2005a) SIKE is an IKKE/TBK1-associated suppressor of TLR3- and virus-triggered IRF-3 activation pathways. 24: 4018-4028.

Huang, J., Wu, S., Barrera, J., Matthews, K. \& Pan, D., (2005b) The Hippo signaling pathway coordinately regulates cell proliferation and apoptosis by inactivating Yorkie, the Drosophila homolog of YAP. Cell 122: 421-434.

Huang, T.Y., Markley, N.A. \& Young, D., (2003) Nak1, an essential germinal center (GC) kinase regulates cell morphology and growth in Schizosaccharomyces pombe. Journal of Biological Chemistry 278: 991997.

Hubbard, M.A. \& Kaminskyj, S.G.W., (2008) Rapid tip-directed movement of Golgi equivalents in growing Aspergillus nidulans hyphae suggests a mechanism for delivery of growth-related materials. Microbiology 154: 1544-1553.

Hwang, J. \& Pallas, D.C., (2014) STRIPAK complexes: Structure, biological function, and involvement in human diseases. The International Journal of Biochemistry \& Cell Biology 47: 118-148.

Hyde, G.J., Davies, D., Perasso, L., Cole, L. \& Ashford, A.E., (1999) Microtubules, but not actin microfilaments, regulate vacuole motility and morphology in hyphae of Pisolithus tinctorius. Cell motility and the cytoskeleton 42: 114-124.

Hyodo, T., Ito, S., Hasegawa, H., Asano, E., Maeda, M., Urano, T., Takahashi, M., Hamaguchi, M. \& Senga, T., (2012) Misshapen-like kinase 1 (MINK1) is a novel component of striatin-interacting phosphatase 


\section{References}

and kinase (STRIPAK) and is required for the completion of cytokinesis. Journal of Biological Chemistry 287: 25019-25029.

Hyttinen, J.M.T., Niittykoski, M., Salminen, A. \& Kaarniranta, K., (2013) Maturation of autophagosomes and endosomes: A key role for Rab7. Biochimica et Biophysica Acta (BBA) - Molecular Cell Research 1833: 503-510.

Iossifov, I., Ronemus, M., Levy, D., Wang, Z., Hakker, I., Rosenbaum, J., Yamrom, B., Lee, Y.-h., Narzisi, G., Leotta, A., Kendall, J., Grabowska, E., Ma, B., Marks, S., Rodgers, L., Stepansky, A., Troge, J., Andrews, P., Bekritsky, M., Pradhan, K., Ghiban, E., Kramer, M., Parla, J., Demeter, R., Fulton, L.L., Fulton, R.S., Magrini, V.J., Ye, K., Darnell, J.C., Darnell, R.B., Mardis, E.R., Wilson, R.K., Schatz, M.C., McCombie, W.R. \& Wigler, M., (2012) De novo gene disruptions in children on the autistic spectrum. Neuron 74: 285-299.

Ishikawa, T., Sato, A., Marcou, C.A., Tester, D.J., Ackerman, M.J., Crotti, L., Schwartz, P.J., On, Y.K., Park, J.E., Nakamura, K., Hiraoka, M., Nakazawa, K., Sakurada, H., Arimura, T., Makita, N. \& Kimura, A., (2012) A novel disease gene for Brugada Syndrome: Sarcolemmal membrane-associated protein gene mutations impair intracellular trafficking of hNav1.5. Circ Arrhythm Electrophysiol. 5: 10981107.

Islam, K.T., Bond, J.P. \& Fakhoury, A.M., (2017) FvSTR1, a striatin orthologue in Fusarium virguliforme, is required for asexual development and virulence. Appl Microbiol Biotechnol 101: 6431-6445.

Jackson, S.L. \& Heath, I.B., (1993) Roles of calcium ions in hyphal tip growth. Microbiological Reviews 57: 367-382.

Jäger, S., Bucci, C., Tanida, I., Ueno, T., Kominami, E., Saftig, P. \& Eskelinen, E.-L., (2004) Role for Rab7 in maturation of late autophagic vacuoles. Journal of Cell Science 117: 4837-4848.

Jain, B.P., Chauhan, P., Tanti, G.K., Singarapu, N., Ghaskadbi, S. \& Goswami, S.K., (2015) Tissue specific expression of SG2NA is regulated by differential splicing, RNA editing and differential polyadenylation. Gene 556: 119-126.

Jain, B.P., Pandey, S., Saleem, N., Tanti, G.K., Mishra, S. \& Goswami, S.K., (2017) SG2NA is a regulator of endoplasmic reticulum (ER) homeostasis as its depletion leads to ER stress. Cell Stress Chaperones: 853-866.

James, P., Halladay, J. \& Craig, E.A., (1996) Genomic libraries and a host strain designed for highly efficient two-hybrid selection in yeast. Genetics 144: 1425-1436.

Janssens, V. \& Goris, J., (2001) Protein phosphatase 2A: a highly regulated family of serine/threonine phosphatases implicated in cell growth and signalling. Biochemical Journal 353: 417-439.

Janssens, V., Longin, S. \& Goris, J., (2008) PP2A holoenzyme assembly: in cauda venenum (the sting is in the tail). Trends in Biochemical Sciences 33: 113-121.

Janus, D., Hoff, B., Hofmann, E. \& Kück, U., (2007) An efficient fungal RNA-silencing system using the Dsred reporter gene. Applied and Environmental Microbiology 73: 962-970.

Jones, T.A., Barker, H.M., da Cruz e Silva, E.F., Mayer-Jaekel, R.E., Hemmings, B.A., Spurr, N.K., Sheer, D. \& Cohen, P.T., (1993) Localization of the genes encoding the catalytic subunits of protein phosphatase 2A to human chromosome bands 5q23-q31 and 8p12-p11.2, respectively. Cytogenet Cell Genet 63: $35-41$.

Jung, C.H., Ro, S.-H., Cao, J., Otto, N.M. \& Kim, D.-H., (2010) mTOR regulation of autophagy. FEBS letters 584: 1287-1295.

Kammerer, R.A., (1997) $\alpha$-Helical coiled-coil oligomerization domains in extracellular proteins. Matrix Biology 15: 555-565.

Kanai, M., Kume, K., Miyahara, K., Sakai, K., Nakamura, K., Leonhard, K., Wiley, D.J., Verde, F., Toda, T. \& Hirata, D., (2005) Fission yeast MO25 protein is localized at SPB and septum and is essential for cell morphogenesis. The EMBO Journal 24: 3012-3025.

Kango-Singh, M. \& Singh, A., (2009) Regulation of organ size: Insights from the Drosophila Hippo signaling pathway. Developmental Dynamics 238: 1627-1637. 


\section{References}

Katoh, K. \& Standley, D.M., (2013) MAFFT multiple sequence alignment software version 7: Improvements in performance and usability. Molecular Biology and Evolution 30: 772-780.

Kawabata, T. \& Inoue, H., (2007) Detection of physical interactions by immunoprecipitation of FLAG - and HA-tagged proteins expressed at the his-3 locus in Neurospora crassa. Fungal Genetics Newsletter 54: 5-8.

Kean, M.J., Ceccarelli, D.F., Goudreault, M., Sanches, M., Tate, S., Larsen, B., Gibson, L.C.D., Derry, W.B., Scott, I.C., Pelletier, L., Baillie, G.S., Sicheri, F. \& Gingras, A.-C., (2011) Structure-function analysis of core STRIPAK proteins: A signaling complex implicated in Golgi polarization The Journal of biological chemistry 286: 25065-25075.

Keen, J.H., (1990) Clathrin and associated assembly and disassembly proteins. Annual Review of Biochemistry 59: 415-438.

Kemp, H.A. \& Sprague, J., George F., (2003) Far3 and five interacting proteins prevent premature recovery from pheromone arrest in the budding yeast Saccharomyces cerevisiae. Molecular and Cellular Biology 23: 1750-1763.

Khew-Goodall, Y. \& Hemmings, B.A., (1988) Tissue-specific expression of mRNAs encoding $\alpha$ - and $\beta$-catalytic subunits of protein phosphatase 2A. FEBS Letters 238: 265-268.

Khew-Goodall, Y., Mayer, R.E., Maurer, F., Stone, S.R. \& Hemmings, B.A., (1991) Structure and transcriptional regulation of protein phosphatase 2A catalytic subunit genes. Biochemistry 30: 8997.

Kilaru, S., Schuster, M., Latz, M., Guo, M. \& Steinberg, G., (2015) Fluorescent markers of the endocytic pathway in Zymoseptoria tritici. Fungal Genetics and Biology 79: 150-157.

Kim, H.-S., Czymmek, K.J., Patel, A., Modla, S., Nohe, A., Duncan, R., Gilroy, S. \& Kang, S., (2012) Expression of the Cameleon calcium biosensor in fungi reveals distinct $\mathrm{Ca}^{2+}$ signatures associated with polarized growth, development, and pathogenesis. Fungal Genetics and Biology 49: 589-601.

Kirby, K.S., (1956) A new method for the isolation of ribonucleic acids from mammalian tissues. Biochemical Journal 64: 405-408.

Kirchhausen, T., (1999) Adaptors for clathrin-mediated traffic. Annual Review of Cell and Developmental Biology 15: 705-732.

Klix, V., Nowrousian, M., Ringelberg, C., Loros, J.J., Dunlap, J.C. \& Pöggeler, S., (2010) Functional characterization of MAT1-1-specific mating-type genes in the homothallic ascomycete Sordaria macrospora provides new insights into essential and non-essential sexual regulators. Eukaryot Cell 9: 894-905.

Krapp, A., Schmidt, S., Cano, E. \& Simanis, V., (2001) S. pombe Cdc11p, together with Sid4p, provides an anchor for septation initiation network proteins on the spindle pole body. Current Biology 11: 15591568.

Krapp, A. \& Simanis, V., (2008) An overview of the fission yeast septation initiation network (SIN). Biochemical Society Transactions 36: 411-415.

Kück, U., Beier, A.M. \& Teichert, I., (2016) The composition and function of the striatin-interacting phosphatases and kinases (STRIPAK) complex in fungi. Fungal Genetics and Biology 90: 31-38.

Kück, U., Pöggeler, S., Nowrousian, M., Nolting, N. \& Engh, I., (2009) Sordaria macrospora, a model system for fungal development. In: The Mycota XV. T. Anke \& D. Weber (eds). Heidelberg: Springer Verlag, pp. 17-39.

Ladinsky, M.S., Mastronarde, D.N., McIntosh, J.R., Howell, K.E. \& Staehelin, L.A., (1999) Golgi structure in three dimensions: Functional insights from the normal rat kidney cell. The Journal of Cell Biology 144: 1135-1149.

Laemmli, U.K., (1970) Cleavage of structural proteins during the assembly of the head of bacteriophage T4. Nature 227: 680-685.

Lai, F., Wu, R., Wang, J., Li, C., Zou, L., Lu, Y. \& Liang, C., (2011) Far3p domains involved in the interactions of far proteins and pheromone-induced cell cycle arrest in budding yeast. 11: 72-79. 


\section{References}

Lai, Z.-C., Wei, X., Shimizu, T., Ramos, E., Rohrbaugh, M., Nikolaidis, N., Ho, L.-L. \& Li, Y., (2005) Control of cell proliferation and apoptosis by mob as tumor suppressor, Mats. Cell 120: 675-685.

Lambright, D.G., Sondek, J., Bohm, A., Skiba, N.P., Hamm, H.E. \& Sigler, P.B., (1996) The $2.0 \AA$ crystal structure of a heterotrimeric $G$ protein. Nature 379: 311-319.

Lant, B., Yu, B., Goudreault, M., Holmyard, D., Knight, J.D.R., Xu, P., Zhao, L., Chin, K., Wallace, E., Zhen, M., Gingras, A.-C. \& Derry, W.B., (2015) CCM-3/STRIPAK promotes seamless tube extension through endocytic recycling. 6: 6449.

Le Borgne, R., Alconada, A., Bauer, U. \& Hoflack, B., (1998) The mammalian AP-3 adaptor-like complex mediates the intracellular transport of lysosomal membrane glycoproteins. Journal of Biological Chemistry 273: 29451-29461.

Leberer, E., Dignard, D., Harcus, D., Thomas, D.Y. \& Whiteway, M., (1992) The protein kinase homologue Ste20p is required to link the yeast pheromone response G-protein beta gamma subunits to downstream signalling components. The EMBO Journal 11: 4815-4824.

Lee, S.C. \& Shaw, B.D., (2008) Localization and function of ADP ribosylation factor A in Aspergillus nidulans. FEMS Microbiology Letters 283: 216-222.

Letunic, I., Doerks, T. \& Bork, P., (2015) SMART: recent updates, new developments and status in 2015. Nucleic Acids Research 43: D257-D260.

Levin, D.E., (2005) Cell wall integrity signaling in Saccharomyces cerevisiae. Microbiology and Molecular Biology Reviews 69: 262-291.

Levin, D.E., (2011) Regulation of cell wall biogenesis in Saccharomyces cerevisiae: The cell wall integrity signaling pathway. Genetics 189: 1145-1175.

Li, C., Furge, K.A., Cheng, Q.-c. \& Albright, C.F., (2000) Byr4 localizes to spindle-pole bodies in a cell cycleregulated manner to control Cdc7 localization and septation in fission yeast. Journal of Biological Chemistry 275: 14381-14387.

Li, J., Mahajan, A. \& Tsai, M.-D., (2006) Ankyrin repeat: A unique motif mediating protein-protein interactions. Biochemistry 45: 15168-15178.

Li, S., Couet, J. \& Lisanti, M.P., (1996) Src tyrosine kinases, G $\alpha$ subunits, and H-Ras share a common membrane-anchored scaffolding protein, caveolin: caveolin binding negatively regulates the autoactivation of Src tyrosine kinases Journal of Biological Chemistry 271: 29182-29190.

Li, S., Okamoto, T., Chun, M., Sargiacomo, M., Casanova, J.E., Hansen, S.H., Nishimoto, I. \& Lisanti, M.P., (1995) Evidence for a regulated interaction between heterotrimeric $G$ proteins and caveolin. Journal of Biological Chemistry 270: 15693-15701.

Lichius, A. \& Read, N.D., (2009) A versatile set of Lifeact-RFP expression plasmids for live-cell imaging of Factin in filamentous fungi. Fungal Genetics Reports 57: 8-14.

Lin, A., Hokugo, A., Choi, J. \& Nishimura, I., (2010) Small cytoskeleton-associated molecule, fibroblast growth factor receptor 1 oncogene partner 2/wound inducible transcript-3.0 (FGFR1OP2/wit3.0), facilitates fibroblast-driven wound closure. The American Journal of Pathology 176: 108-121.

Lisa-Santamaría, P., Jiménez, A. \& Revuelta, J.L., (2012) The protein factor-arrest 11 (Far11) Is essential for the toxicity of human caspase-10 in yeast and participates in the regulation of autophagy and the DNA damage signaling. The Journal of Biological Chemistry 287: 29636-29647.

Logan, C.Y.a.N., Roel (2004) The Wnt signaling pathway in development and disease Annual Review of Cell and Developmental Biology 20: 781-810.

López-Sánchez, I., Sanz-García, M. \& Lazo, P.A., (2009) Plk3 Interacts with and specifically phosphorylates VRK1 in Ser ${ }^{342}$, a downstream target in a pathway that induces Golgi fragmentation. Molecular and Cellular Biology 29: 1189-1201.

Lord, K.M. \& Read, N.D., (2011) Perithecium morphogenesis in Sordaria macrospora. Fungal Genetics and Biology 48: 388-399.

Lu, Q., Pallas, D.C., Surks, H.K., Baur, W.E., Mendelsohn, M.E. \& Karas, R.H., (2004) Striatin assembles a membrane signaling complex necessary for rapid, nongenomic activation of endothelial NO 


\section{References}

synthase by estrogen receptor $\alpha$. Proceedings of the National Academy of Sciences of the United States of America 101: 17126-17131.

Lu, W. \& Gelfand, V.I., (2017) Moonlighting motors: Kinesin, dynein, and cell polarity. Trends in Cell Biology 27: 505-514.

Luca, F.C. \& Winey, M., (1998) MOB1, an essential yeast gene required for completion of mitosis and maintenance of ploidy. Molecular Biology of the Cell 9: 29-46.

Lucas, E.P., Khanal, I., Gaspar, P., Fletcher, G.C., Polesello, C., Tapon, N. \& Thompson, B.J., (2013) The Hippo pathway polarizes the actin cytoskeleton during collective migration of Drosophila border cells. The Journal of Cell Biology 201: 875-885.

Lupas, A., (1996) Methods in Enzymology. In: Prediction and analysis of coiled-coil structures. F.D. Russell (ed). Academic Press, pp. 513-525.

Lupas, A., Van Dyke, M. \& Stock, J., (1991) Predicting coiled coils from protein sequences. Science 252: 11621164.

Ma, X., Chen, Y., Xu, W., Wu, N., Li, M., Cao, Y., Wu, S., Li, Q. \& Xue, L., (2015) Impaired Hippo signaling promotes Rho1-JNK-dependent growth. Proceedings of the National Academy of Sciences of the United States of America 112: 1065-1070.

Ma, X., Zhao, H., Shan, J., Long, F., Chen, Y., Chen, Y., Zhang, Y., Han, X. \& Ma, D., (2007) PDCD10 interacts with Ste20-related kinase MST4 to promote cell growth and transformation via modulation of the ERK pathway. Molecular Biology of the Cell 18: 1965-1978.

Maerz, S., Dettmann, A., Ziv, C., Liu, Y., Valerius, O., Yarden, O. \& Seiler, S., (2009) Two NDR kinase-MOB complexes function as distinct modules during septum formation and tip extension in Neurospora crassa. Molecular Microbiology 74: 707-723.

Marion, J.D., Roberts, C.F., Call, R.J., Forbes, J.L., Nelson, K.T., Bell, J.E. \& Bell, J.K., (2013) Mechanism of endogenous regulation of the type I interferon response by suppressor of IKB kinase $\epsilon$ (SIKE), a novel substrate of TANK-binding kinase 1 (TBK1). The Journal of Biological Chemistry 288: 18612-18623.

Masloff, S., Pöggeler, S. \& Kück, U., (1999) The pro1 gene from Sordaria macrospora encodes a C6 zinc finger transcription factor required for fruiting-body development. Genetics 152: 191-199.

Matsuki, T., Chen, J. \& Howell, B.W., (2013) Acute inactivation of the serine-threonine kinase Stk25 disrupts neuronal migration. Neural Development 8: 21-21.

Mayrhofer, S., Weber, J.M. \& Pöggeler, S., (2006) Pheromones and pheromone receptors are required for proper sexual development in the homothallic ascomycete Sordaria macrospora. Genetics 172: 1521-1533.

McCollum, D. \& Gould, K.L., (2001) Timing is everything: regulation of mitotic exit and cytokinesis by the MEN and SIN. Trends in Cell Biology 11: 89-95.

McCubrey, J.A., Steelman, L.S., Chappell, W.H., Abrams, S.L., Wong, E.W.T., Chang, F., Lehmann, B., Terrian, D.M., Milella, M., Tafuri, A., Stivala, F., Libra, M., Basecke, J., Evangelisti, C., Martelli, A.M. \& Franklin, R.A., (2007) Roles of the Raf/MEK/ERK pathway in cell growth, malignant transformation and drug resistance Biochimica et biophysica acta 1773: 1263-1284.

McLachlan, A.D. \& Stewart, M., (1975) Tropomyosin coiled-coil interactions: Evidence for an unstaggered structure. Journal of Molecular Biology 98: 293-304.

Meitinger, F., Palani, S. \& Pereira, G., (2012) The power of MEN in cytokinesis. Cell Cycle 11: 219-228.

Meitinger, F., Petrova, B., Lombardi, I.M., Bertazzi, D.T., Hub, B., Zentgraf, H. \& Pereira, G., (2010) Targeted localization of Inn1, Cyk3 and Chs2 by the mitotic-exit network regulates cytokinesis in budding yeast. Journal of Cell Science 123: 1851-1861.

Menssen, R., Neutzner, A. \& Seufert, W., (2001) Asymmetric spindle pole localization of yeast Cdc15 kinase links mitotic exit and cytokinesis. Current Biology 11: 345-350.

Meurs, K.M., Mauceli, E., Lahmers, S., Acland, G.M., White, S.N. \& Lindblad-Toh, K., (2010) Genome-wide association identifies a deletion in the $3^{\prime}$ untranslated region of striatin in a canine model of arrhythmogenic right ventricular cardiomyopathy: Identification of striatin deletion in canine ARVC. Human Genetics 128: 315-324. 


\section{References}

Midgley, C.A., White, S., Howitt, R., Save, V., Dunlop, M.G., Hall, P.A., Lane, D.P., Wyllie, A.H. \& Bubb, V.J., (1997) APC expression in normal human tissues. The Journal of Pathology 181: 426-433.

Minet, M., Nurse, P., Thuriaux, P. \& Mitchison, J.M., (1979) Uncontrolled septation in a cell division cycle mutant of the fission yeast Schizosaccharomyces pombe. Journal of Bacteriology 137: 440-446.

Mogelsvang, S., Marsh, B.J., Ladinsky, M.S. \& Howell, K.E., (2004) Predicting function from structure: 3D structure studies of the mammalian Golgi complex. Traffic 5: 338-345.

Mohl, D.A., Huddleston, M.J., Collingwood, T.S., Annan, R.S. \& Deshaies, R.J., (2009) Dbf2-Mob1 drives relocalization of protein phosphatase $\mathrm{Cdc} 14$ to the cytoplasm during exit from mitosis. The Journal of Cell Biology 184: 527-539.

Moon, R.T., Kohn, A.D., Ferrari, G.V.D. \& Kaykas, A., (2004) Wnt and beta-catenin signalling: diseases and therapies. Nat Rev Genet 5: 691-701.

Moreno, C.S., Lane, W.S. \& Pallas, D.C., (2001) A mammalian homolog of yeast MOB1 is both a member and a putative substrate of striatin family-protein phosphatase $2 \mathrm{~A}$ complexes. The Journal of biological chemistry 276: 24253-24260.

Moreno, C.S., Park, S., Nelson, K., Ashby, D., Hubalek, F., Lane, W.S. \& Pallas, D.C., (2000) WD40 repeat proteins striatin and $S / G(2)$ nuclear autoantigen are members of a novel family of calmodulinbinding proteins that associate with protein phosphatase 2A. The Journal of biological chemistry 275: 5257-5263.

Mullis, K.B. \& Fallona, F.A., (1987) Specific synthesis of DNA in vitro via polymerase catalysed chain reaction. Meth Enzym 155: 335-350.

Munro, S., (2011) The Golgin coiled-coil proteins of the Golgi apparatus. Cold Spring Harbor Perspectives in Biology 3: a005256.

Muro, Y., Chan, E.K.L., Landberg, G. \& Tan, E.M., (1995) A cell-cycle nuclear autoantigen containing WD-40 motifs expressed mainly in $\mathrm{S}$ and $\mathrm{G}_{2}$-phase cells. Biochemical and Biophysical Research Communications 207: 1029-1037.

Musacchio, A., Gibson, T., Lehto, V.-P. \& Saraste, M., (1992) SH3 - an abundant protein domain in search of a function. FEBS Letters 307: 55-61.

Nader, M., Westendorp, B., Hawari, O., Salih, M., Stewart, A.F.R., Leenen, F.H.H. \& Tuana, B.S., (2012) Tailanchored membrane protein SLMAP is a novel regulator of cardiac function at the sarcoplasmic reticulum. American Journal of Physiology - Heart and Circulatory Physiology 302: H1138-H1145.

Neer, E.J., Schmidt, C.J., Nambudripad, R. \& Smith, T.F., (1994) The ancient regulatory-protein family of WDrepeat proteins. Nature 371: 297-300.

Neisch, A.L., Neufeld, T.P. \& Hays, T.S., (2017) A STRIPAK complex mediates axonal transport of autophagosomes and dense core vesicles through PP2A regulation. The Journal of Cell Biology: $10.1083 /$ jcb.201606082.

Nelson, W.J. \& Nusse, R., (2004) Convergence of Wnt, $\beta$-catenin, and cadherin pathways. Science (New York, N.y.) 303: 1483-1487.

Newport, J.W. \& Forbes, D.J., (1987) The Nucleus: Structure, function, and dynamics. Annual Review of Biochemistry 56: 535-565.

Nicholas, K.B. \& Nicholas, H.B.J., (1997) GeneDoc: a tool for editing and annotating multiple sequence alignments.

Nie, Z., Hirsch, D.S. \& Randazzo, P.A., (2003) Arf and its many interactors. Current Opinion in Cell Biology 15: 396-404.

Nogueira, E., Fidalgo, M., Molnar, A., Kyriakis, J., Force, T., Zalvide, J. \& Pombo, C.M., (2008) SOK1 translocates from the Golgi to the nucleus upon chemical anoxia and induces apoptotic cell death. The Journal of biological chemistry 283: 16248-16258.

Nolting, N. \& Pöggeler, S., (2006) A MADS box protein interacts with a mating-type protein and is required for fruiting-body development in the homothallic ascomycete Sordaria macrospora. Eukaryot Cell 5: 1043-1056. 


\section{References}

Nomoto, S., Nakayama, N., Arai, K. \& Matsumoto, K., (1990) Regulation of the yeast pheromone response pathway by $\mathrm{G}$ protein subunits. The EMBO Journal 9: 691-696.

Nordzieke, S., (2014) Molecular genetic studies of PRO45 in Sordaria macrospora: A homolog of the sarcolemmal membrane-associated protein (SLMAP) localizes to the nuclear envelope and plays an essential role in fungal development. General and Molecular Botany: 1-100.

Nordzieke, S., Zobel, T., Fränzel, B., Wolters, D.A., Kück, U. \& Teichert, I., (2015) A fungal sarcolemmal membrane-associated protein (SLMAP) homolog plays a fundamental role in development and localizes to the nuclear envelope, endoplasmic reticulum, and mitochondria. Eukaryotic Cell 14: 345358.

Nowrousian, M., (2010) Next-generation sequencing techniques for eukaryotic microorganisms: sequencing-based solutions to biological problems. Eukaryot Cell 9: 1300-1310.

Nowrousian, M. \& Cebula, P., (2005) The gene for a lectin-like protein is transcriptionally activated during sexual development, but is not essential for fruiting-body formation in the filamentous fungus Sordaria macrospora. BMC Microbiology 5: 1-10.

Nowrousian, M., Frank, S., Koers, S., Strauch, P., Weitner, T., Ringelberg, C., Dunlap, J.C., Loros, J.J. \& Kück, U., (2007) The novel ER membrane protein PRO41 is essential for sexual development in the filamentous fungus Sordaria macrospora. Mol Microbiol 64: 923-937.

Nowrousian, M., Masloff, S., Pöggeler, S. \& Kück, U., (1999) Cell differentiation during sexual development of the fungus Sordaria macrospora requires ATP citrate lyase activity. Mol Cell Biol 19.

Nowrousian, M., Stajich, J.E., Chu, M., Engh, I., Espagne, E., Halliday, K., Kamerewerd, J., Kempken, F., Knab, B., Kuo, H.C., Osiewacz, H.D., Pöggeler, S., Read, N.D., Seiler, S., Smith, K.M., Zickler, D., Kück, U. \& Freitag, M., (2010) De novo assembly of a $40 \mathrm{Mb}$ eukaryotic genome from short sequence reads: Sordaria macrospora, a model organism for fungal morphogenesis. PLOS Genet 6: e1000891.

Nowrousian, M., Teichert, I., Masloff, S. \& Kück, U., (2012) Whole-genome sequencing of Sordaria macrospora mutants identifies developmental genes. G3: Genes/Genomes/Genetics 2: 261-270.

Nunbhakdi-Craig, V., Schuechner, S., Sontag, J.-M., Montgomery, L., Pallas, D.C., Juno, C., Mudrak, I., Ogris, E. \& Sontag, E., (2007) Expression of protein phosphatase 2A mutants and silencing of the regulatory $\mathrm{B} \alpha$ subunit induce a selective loss of acetylated and detyrosinated microtubules. Journal of Neurochemistry 101: 959-971.

Ohkura, H., Hagan, I.M. \& Glover, D.M., (1995) The conserved Schizosaccharomyces pombe kinase Plo1, required to form a bipolar spindle, the actin ring, and septum, can drive septum formation in $\mathrm{G} 1$ and G2 cells. Genes \& Development 9: 1059-1073.

Ohoka, Y. \& Takai, Y., (1998) Isolation and characterization of cortactin isoforms and a novel cortactinbinding protein, CBP90. Genes to Cells 3: 603-612.

Orlean, P. \& Menon, A.K., (2007) Thematic review series: Lipid posttranslational modifications. GPI anchoring of protein in yeast and mammalian cells, or: how we learned to stop worrying and love glycophospholipids. Journal of Lipid Research 48: 993-1011.

Pace, N.R., (2009) Mapping the tree of life: Progress and prospects. Microbiology and Molecular Biology Reviews : MMBR 73: 565-576.

Page, R.D.M., (1996) Tree View: An application to display phylogenetic trees on personal computers. Computer applications in the Biosciences: CABIOS 12: 357-358.

Pal, S., Lant, B., Yu, B., Tian, R., Tong, J., Krieger, J.R., Moran, M.F., Gingras, A.-C. \& Derry, W.B., (2017) CCM3 promotes $C$. elegans germline development by regulating vesicle trafficking cytokinesis and polarity. Current Biology 27: 868-876.

Pantazopoulou, A., Pinar, M., Xiang, X. \& Peñalva, M.A., (2014) Maturation of late Golgi cisternae into RabE RAB11 exocytic post-Golgi carriers visualized in vivo. Molecular Biology of the Cell 25: 2428-2443.

Park, Sang Y. \& Guo, X., (2014) Adaptor protein complexes and intracellular transport. Bioscience Reports 34: e00123.

Parton, R.G. \& Simons, K., (2007) The multiple faces of caveolae. Nat Rev Mol Cell Biol 8: 185-194. 


\section{References}

Peñalva, M.A., (2005) Tracing the endocytic pathway of Aspergillus nidulans with FM4-64. Fungal Genetics and Biology 42: 963-975.

Peñalva, M.Á., (2010) Endocytosis in filamentous fungi: Cinderella gets her reward. Current Opinion in Microbiology 13: 684-692.

Peñalva, M.A., Zhang, J., Xiang, X. \& Pantazopoulou, A., (2017) Transport of fungal RAB11 secretory vesicles involves myosin-5, dynein/dynactin/p25, and kinesin-1 and is independent of kinesin-3. Molecular Biology of the Cell 28: 947-961.

Penzes, P. \& Cahill, M.E., (2012) Deconstructing signal transduction pathways that regulate the actin cytoskeleton in dendritic spines. Cytoskeleton (Hoboken, N.J.) 69: 426-441.

Petralia, R.S., Wang, Y.-X. \& Wenthold, R.J., (2003) Internalization at glutamatergic synapses during development. European Journal of Neuroscience 18: 3207-3217.

Pfaffl, M.W., Horgan, G.W. \& Dempfle, L., (2002) Relative expression software tool (RESTC) for group-wise comparison and statistical analysis of relative expression results in real-time PCR. Nucleic Acids Research 30: e36.

Pillet, V., Zehnder, M., Seewald, A.K., Veuthey, A.-L. \& Petrak, J., (2005) GPSDB: A new database for synonyms expansion of gene and protein names. Bioinformatics 21: 1743-1744.

Piper, R.C., Cooper, A.A., Yang, H. \& Stevens, T.H., (1995) VPS27 controls vacuolar and endocytic traffic through a prevacuolar compartment in Saccharomyces cerevisiae. The Journal of Cell Biology 131: 603-617.

Pöggeler, S., (2000) Sexualdifferenzierung bei Pilzen. Habilitationsschrift. Ruhr-Universität Bochum

Pöggeler, S. \& Kück, U., (2004) A WD40 repeat protein regulates fungal cell differentiation and can be replaced functionally by the mammalian homologue striatin. Eukaryotic Cell 3: 232-240.

Pöggeler, S. \& Kück, U., (2006) Highly efficient generation of signal transduction knockout mutants using a fungal strain deficient in the mammalian ku70 ortholog. Gene 378: 1-10.

Pöggeler, S., Masloff, S., Hoff, B., Mayrhofer, S. \& Kück, U., (2003) Versatile EGFP reporter plasmids for cellular localization of recombinant gene products in filamentous fungi. Current Genetics 43: 54-61.

Pöggeler, S., Nowrousian, M., Jacobsen, S. \& Kück, U., (1997) An efficient procedure to isolate fungal genes from an indexed cosmid library. Journal of Microbiological Methods 29: 49-61.

Pöggeler, S., Nowrousian, M. \& Kück, U., (2006) The Mycota I. In: Fruiting-body development in ascomycetes. U. Kües \& R. Fischer (eds). Berlin, Heidelberg: Springer.

Pombo, C.M., Bonventre, J.V., Molnar, A., Kyriakis, J. \& Force, T., (1996) Activation of a human Ste20-like kinase by oxidant stress defines a novel stress response pathway. The EMBO Journal 15: 4537-4546.

Pombo, C.M., Tsujita, T., Kyriakis, J.M., Bonventre, J.V. \& Force, T., (1997) Activation of the Ste20-like oxidant stress response kinase-1 during the initial stages of chemical anoxia-induced necrotic cell death: Requirement for dual inputs of oxidant stress and increased cytosolic $\mathrm{Ca}^{2+}$. Journal of Biological Chemistry 272: 29372-29379.

Ponchon, L., Dumas, C., Fesquet, D. \& Padilla, A., (2004) Resonance assignments and topology of the 15N, 13C labelled 23 kDa core domain of Xenopus Mob1. 28: 299-300.

Popova, N.V., Deyev, I.E. \& Petrenko, A.G., (2013) Clathrin-mediated endocytosis and adaptor proteins. Acta Naturae 5: 62-73.

Pracheil, T. \& Liu, Z., (2013) Tiered assembly of the yeast far3-7-8-9-10-11 complex at the endoplasmic reticulum. Journal of Biological Chemistry 288: 16986-16997.

Pracheil, T., Thornton, J. \& Liu, Z., (2012) TORC2 signaling is antagonized by protein phosphatase $2 \mathrm{~A}$ and the Far complex in Saccharomyces cerevisiae. Genetics 190: 1325-1339.

Preisinger, C., Short, B., De Corte, V., Bruyneel, E., Haas, A., Kopajtich, R., Gettemans, J. \& Barr, F.A., (2004) YSK1 is activated by the Golgi matrix protein GM130 and plays a role in cell migration through its substrate 14-3-33. The Journal of Cell Biology 164: 1009-1020.

Prinz, W.A., Grzyb, L., Veenhuis, M., Kahana, J.A., Silver, P.A. \& Rapoport, T.A., (2000) Mutants affecting the structure of the cortical endoplasmic reticulum in Saccharomyces cerevisiae. The Journal of Cell Biology 150: 461-474. 


\section{References}

Rappsilber, J., Ishihama, Y. \& Mann, M., (2003) Stop and go extraction tips for matrix-assisted laser desorption/ionization, nanoelectrospray, and LC/MS sample pretreatment in proteomics. Anal Chem 75: 663-670.

Rappsilber, J., Mann, M. \& Ishihama, Y., (2007) Protocol for micro-purification, enrichment, prefractionation and storage of peptides for proteomics using StageTips. Nature protocols 2: 18961906.

Ravikumar, B., Imarisio, S., Sarkar, S., O'Kane, C.J. \& Rubinsztein, D.C., (2008) Rab5 modulates mutant huntingtin aggregation/toxicity in cell and fly models via macroautophagy. Journal of cell science 121: 1649-1660.

Razi, M., Chan, E.Y.W. \& Tooze, S.A., (2009) Early endosomes and endosomal coatomer are required for autophagy. The Journal of Cell Biology 185: 305-321.

Read, N.D., Fischer, S. \& Parton, R.M., (1998) Imaging Spitzenkörper, pH and calcium dynamics in growing fungal hyphae. Pesticide Science 54: 179-181.

Rech, C., Engh, I. \& Kück, U., (2007) Detection of hyphal fusion in filamentous fungi using differently fluorescence-labeled histones. Current Genetics 52: 259-266.

Ribeiro, P.S., Josué, F., Wepf, A., Wehr, M.C., Rinner, O., Kelly, G., Tapon, N. \& Gstaiger, M., (2010) Combined functional genomic and proteomic approaches identify a PP2A complex as a negative regulator of Hippo signaling. Molecular Cell 39: 521-534.

Riedl, J., Crevenna, A.H., Kessenbrock, K., Yu, J.H., Neukirchen, D., Bista, M., Bradke, F., Jenne, D., Holak, T.A., Werb, Z., Sixt, M. \& Wedlich-Soldner, R., (2008) Lifeact: a versatile marker to visualize F-actin. Nature Methods 5: 605.

Ríos-Barrera, L.D. \& Riesgo-Escovar, J.R., (2013) Regulating cell morphogenesis: The Drosophila jun Nterminal kinase pathway. genesis 51: 147-162.

Robinson, M.S., (1992) Adaptins. Trends in Cell Biology 2: 293-297.

Rosenberg, J.A., Tomlin, G.C., McDonald, W.H., Snydsman, B.E., Muller, E.G., Yates, J.R. \& Gould, K.L., (2006) Ppc89 links multiple proteins, including the septation initiation network, to the core of the fission yeast spindle-pole body. Molecular Biology of the Cell 17: 3793-3805.

Ruan, Q., Wang, Q., Xie, S., Fang, Y., Darzynkiewicz, Z., Guan, K., Jhanwar-Uniyal, M. \& Dai, W., (2004) Pololike kinase 3 is Golgi localized and involved in regulating Golgi fragmentation during the cell cycle. Experimental Cell Research 294: 51-59.

Ruediger, R., Van Wart Hood, J.E., Mumby, M. \& Walter, G., (1991) Constant expression and activity of protein phosphatase 2A in synchronized cells. Molecular and Cellular Biology 11: 4282-4285.

Ruvolo, P.P., Deng, X., Ito, T., Carr, B.K. \& May, W.S., (1999) Ceramide induces Bcl2 dephosphorylation via a mechanism involving mitochondrial PP2A. Journal of Biological Chemistry 274: 20296-20300.

Sakuma, C., Kawauchi, T., Haraguchi, S., Shikanai, M., Yamaguchi, Y., Gelfand, V.I., Luo, L., Miura, M. \& Chihara, T., (2014) Drosophila Strip serves as a platform for early endosome organization during axon elongation. Nature communications 5: 5180-5180.

Salimova, E., Sohrmann, M., Fournier, N. \& Simanis, V., (2000) The S. pombe orthologue of the S. cerevisiae MOB1 gene is essential and functions in signalling the onset of septum formation. Journal of Cell Science 113: 1695-1704.

Sambrook, J., Fritsch, E.F. \& Maniatis, T., ( 2001) Moleculare Cloning: A Laboratory Manual. Ed. 2. Cold Spring Habor Press, Cold Spring Habor, NY.

Sambrook, J. \& Russell, D., (2001) Molecular Cloning: A Laboratory Manual. Cold Spring Harbor Laboratory Press.

Sanghamitra, M., Talukder, I., Singarapu, N., Sindhu, K.V., Kateriya, S. \& Goswami, S.K., (2008) WD-40 repeat protein SG2NA has multiple splice variants with tissue restricted and growth responsive properties. Gene 420: 48-56.

Santarpia, L., Lippman, S.L. \& El-Naggar, A.K., (2012) Targeting the mitogen-activated protein kinase RASRAF signaling pathway in cancer therapy. Expert opinion on therapeutic targets 16: 103-119. 


\section{References}

Santoro, M.F., Annand, R.R., Robertson, M.M., Peng, Y.-W., Brady, M.J., Mankovich, J.A., Hackett, M.C., Ghayur, T., Walter, G., Wong, W.W. \& Giegel, D.A., (1998) Regulation of protein phosphatase 2A activity by caspase-3 during apoptosis. Journal of Biological Chemistry 273: 13119-13128.

Schmelzle, T. \& Hall, M.N., (2000) TOR, a central controller of cell growth. Cell 103: 253-262.

Schmid, S.L., (1997) Clathrin-coated vesicle formation and protein sorting:An Integrated Process. Annual Review of Biochemistry 66: 511-548.

Schmidt, S., Sohrmann, M., Hofmann, K., Woollard, A. \& Simanis, V., (1997) The Spg1p GTPase is an essential, dosage-dependent inducer of septum formation in Schizosaccharomyces pombe. Genes \& Development 11: 1519-1534.

Schulte, J., Sepp, K.J., Jorquera, R.A., Wu, C., Song, Y., Hong, P. \& Littleton, J.T., (2010) DMob4/Phocein regulates synapse formation, axonal transport, and microtubule organization. The Journal of neuroscience : the official journal of the Society for Neuroscience 30: 5189-5203.

Schultz, J., Milpetz, F., Bork, P. \& Ponting, C.P., (1998) SMART, a simple modular architecture research tool: Identification of signaling domains. Proceedings of the National Academy of Sciences 95: 5857-5864.

Shaw, B.D., Chung, D.-W., Wang, C.-L., Quintanilla, L.A. \& Upadhyay, S., (2011) A role for endocytic recycling in hyphal growth. Fungal Biology 115: 541-546.

Shevchenko, A., Wilm, M., Vorm, O. \& Mann, M., (1996) Mass spectrometric sequencing of proteins silverstained polyacrylamide gels. Anal Chem 68: 850-858.

Shi, Z., Jiao, S. \& Zhou, Z., (2016) STRIPAK complexes in cell signaling and cancer. Oncogene 35: 4549-4557.

Shim, W.-B., Sagaram, U.S., Choi, Y.-E., So, J., Wilkinson, H.H. \& Lee, Y.-W., (2006) FSR1 is essential for virulence and female fertility in Fusarium verticillioides and F. graminearum. Molecular PlantMicrobe Interactions 19: 725-733.

Shorter, J. \& Warren, G., (2002) Golgi Architecture and inheritance. Annual Review of Cell and Developmental Biology 18: 379-420.

Silverman-Gavrila, L.B. \& Lew, R.R., (2001) Regulation of the tip-high $\left[\mathrm{Ca}^{2+}\right.$ ] gradient in growing hyphae of the fungus Neurospora crassa. European Journal of Cell Biology 80: 379-390.

Simonin, A.R., Rasmussen, C.G., Yang, M. \& Glass, N.L., (2010) Genes encoding a striatin-like protein (HAM3 ) and a forkhead associated protein (HAM-4) are required for hyphal fusion in Neurospora crassa. Fungal Genetics and Biology 47: 855-868.

Simons, K. \& Toomre, D., (2000) Lipid rafts and signal transduction. Nat Rev Mol Cell Biol 1: 31-39.

Simonsen, A. \& Tooze, S.A., (2009) Coordination of membrane events during autophagy by multiple class III PI3-kinase complexes. The Journal of Cell Biology 186: 773-782.

Singh, N.S., Shao, N., McLean, J.R., Sevugan, M., Ren, L., Chew, T.G., Bimbo, A., Sharma, R., Tang, X., Gould, K.L. \& Balasubramanian, M.K., (2011) SIN-inhibitory phosphatase complex (SIP) promotes Cdc11p dephosphorylation and propagates SIN asymmetry in fission yeast. Current biology 21: 1968-1978.

Son, S. \& Osmani, S.A., (2009) Analysis of all protein phosphatase genes in Aspergillus nidulans identifies a new mitotic regulator, Fcp1. Eukaryotic Cell 8: 573-585.

Sondek, J., Bohm, A., Lambright, D.G., Hamm, H.E. \& Sigler, P.B., (1996) Crystal structure of a $G_{A}$ protein Bydimer at $2.1 \AA ̊$ resolution. Nature 379: 369-374.

Song, K.S., Li, S., Okamoto, T., Quilliam, L.A., Sargiacomo, M. \& Lisanti, M.P., (1996) Co-purification and direct Interaction of Ras with caveolin, an integral membrane protein of caveolae microdomains: Detergent-free purification of caveolae membranes Journal of Biological Chemistry 271: 9690-9697.

Southern, E.M., (1975) Detection of specific sequences among DNA fragments separated by gel electrophoresis. J Mol Biol 98: 503-517.

Sparks, C.A., Morphew, M. \& McCollum, D., (1999) Sid2p, a spindle pole body kinase that regulates the onset of cytokinesis. The Journal of Cell Biology 146: 777-790.

Speicher, K., Kolbas, O., Harper, S. \& Speicher, D., (2000) Systematic analysis of peptide recoveries from ingel digestions for protein identifications in proteome studies. Journal of Biomolecular Techniques : JBT 11: 74-86. 


\section{References}

Stavridi, E.S., Harris, K.G., Huyen, Y., Bothos, J., Verwoerd, P.-M., Stayrook, S.E., Pavletich, N.P., Jeffrey, P.D. \& Luca, F.C., (2003) Crystal structure of a human Mob1 protein: Toward understanding Mobregulated cell cycle pathways. Structure 11: 1163-1170.

Steinberg, G., (2007a) Hyphal Growth: a Tale of Motors, Lipids, and the Spitzenkörper. Eukaryotic Cell 6: 351-360.

Steinberg, G., (2007b) On the move: endosomes in fungal growth and pathogenicity. Nat Rev Micro 5: 309316.

Steinberg, G., Schliwa, M., Lehmler, C., Bolker, M., Kahmann, R. \& Mclntosh, J.R., (1998) Kinesin from the plant pathogenic fungus Ustilago maydis is involved in vacuole formation and cytoplasmic migration. Journal of Cell Science 111: 2235-2246.

Su, Y.-C., Treisman, J.E. \& Skolnik, E.Y., (1998) The Drosophila Ste20-related kinase misshapen is required for embryonic dorsal closure and acts through a JNK MAPK module on an evolutionarily conserved signaling pathway. Genes \& Development 12: 2371-2380.

Suda, Y. \& Nakano, A., (2012) The yeast Golgi apparatus. Traffic 13: 505-510.

Sugden, Peter H., McGuffin, Liam J. \& Clerk, A., (2013) SOcK, MiSTs, MASK and STicKs: the GCKIII (germinal centre kinase III) kinases and their heterologous protein-protein interactions. Biochemical Journal 454: 13-30.

Surkont, J. \& Pereira-Leal, J.B., (2015) Evolutionary patterns in coiled-coils. Genome Biology and Evolution 7: 545-556.

Sütterlin, C., Hsu, P., Mallabiabarrena, A. \& Malhotra, V., (2002) Fragmentation and dispersal of the pericentriolar Golgi complex is required for entry into Mitosis in mammalian cells. Cell 109: 359-369.

Sütterlin, C., Lin, C.-Y., Feng, Y., Ferris, D.K., Erikson, R.L. \& Malhotra, V., (2001) Polo-like kinase is required for the fragmentation of pericentriolar Golgi stacks during mitosis. Proceedings of the National Academy of Sciences of the United States of America 98: 9128-9132.

Swanson, S.J., Bethke, P.C. \& Jones, R.L., (1998) Barley aleurone cells contain two types of vacuoles: Characterization of lytic organelles by use of fluorescent probes. The Plant Cell 10: 685-698.

Takeshige, K., Baba, M., Tsuboi, S., Noda, T. \& Ohsumi, Y., (1992) Autophagy in yeast demonstrated with proteinase-deficient mutants and conditions for its induction. The Journal of Cell Biology 119: 301311.

Takeshita, N., Evangelinos, M., Zhou, L., Serizawa, T., Somera-Fajardo, R.A., Lu, L., Takaya, N., Nienhaus, G.U. \& Fischer, R., (2017) Pulses of $\mathrm{Ca}^{2+}$ coordinate actin assembly and exocytosis for stepwise cell extension. Proceedings of the National Academy of Sciences 114: 5701-5706.

Tanaka, K., Petersen, J., Maclver, F., Mulvihill, D.P., Glover, D.M. \& Hagan, I.M., (2001) The role of Plo1 kinase in mitotic commitment and septation in Schizosaccharomyces pombe. The EMBO Journal 20: 12591270.

Tang, D., Mar, K., Warren, G. \& Wang, Y., (2008) Molecular mechanism of mitotic Golgi disassembly and reassembly revealed by a defined reconstitution assay. Journal of Biological Chemistry 283: 60856094.

Tapon, N., Harvey, K.F., Bell, D.W., Wahrer, D.C.R., Schiripo, T.A., Haber, D.A. \& Hariharan, I.K., (2002) Salvador promotes both cell cycle exit and apoptosis in Drosophila and is mutated in human cancer cell lines. Cell 110: 467-478.

Teichert, I., Nowrousian, M., Pöggeler, S. \& Kück, U., (2014) Chapter Four - The filamentous fungus Sordaria macrospora as a genetic model to study fruiting-body development. In: Advances in Genetics. J.C.D. Theodore Friedmann \& F.G. Stephen (eds). Academic Press, pp. 199-244.

Teichert, I., Wolff, G., Kück, U. \& Nowrousian, M., (2012) Combining laser microdissection and RNA-seq to chart the transcriptional landscape of fungal development. BMC Genomics 13: 511.

Theisen, U., Straube, A. \& Steinberg, G., (2008) Dynamic rearrangement of nucleoporins during fungal "open" mitosis. Molecular Biology of the Cell 19: 1230-1240. 


\section{References}

Towbin, H., Staehelin, T. \& Gordon, J., (1979) Electrophoretic transfer of proteins from polyacrylamide gels to nitrocellulose sheets: procedure and some applications. Proceedings of the National Academy of Sciences of the United States of America 76: 4350-4354.

Tucker, C.L., Peteya, L.A., Pittman, A.M.C. \& Zhong, J., (2009) A genetic test for yeast two-hybrid bait competency using RanBPM. Genetics 182: 1377-1379.

Tyanova, S., Temu, T., Sinitcyn, P., Carlson, A., Hein, M.Y., Geiger, T., Mann, M. \& Cox, J., (2016) The Perseus computational platform for comprehensive analysis of (prote)omics data. Nat Meth 13: 731-740.

Van der Klei, I.J. \& Veenhuis, M., (2006) Yeast and filamentous fungi as model organisms in microbody research. Biochimica et Biophysica Acta (BBA) - Molecular Cell Research 1763: 1364-1373.

van der Vaart, A., Griffith, J. \& Reggiori, F., (2010) Exit from the Golgi is required for the expansion of the autophagosomal phagophore in yeast Saccharomyces cerevisiae. Molecular Biology of the Cell 21: 2270-2284.

Vanlandingham, P.A. \& Ceresa, B.P., (2009) Rab7 regulates late endocytic trafficking downstream of multivesicular body biogenesis and cargo sequestration. The Journal of Biological Chemistry 284: 12110-12124.

Verschueren, E., Spiess, M., Gkourtsa, A., Avula, T., Landgraf, C., Mancilla, V.T., Huber, A., Volkmer, R., Winsor, B., Serrano, L., Hochstenbach, F. \& Distel, B., (2015) Evolution of the SH3 domain specificity landscape in yeasts. PLOS ONE 10: e0129229.

Vida, T.A. \& Emr, S.D., (1995) A new vital stain for visualizing vacuolar membrane dynamics and endocytosis in yeast. The Journal of Cell Biology 128: 779-792.

Vitulo, N., Vezzi, A., Galla, G., Citterio, S., Marino, G., Ruperti, B., Zermiani, M., Albertini, E., Valle, G. \& Barcaccia, G., (2007) Characterization and evolution of the cell cycle-associated Mob domaincontaining proteins in eukaryotes. Evolutionary Bioinformatics Online 3: 121-158.

Voigt, O. \& Pöggeler, S., (2013) Autophagy genes Smatg8 and Smatg4 are required for fruiting-body development, vegetative growth and ascospore germination in the filamentous ascomycete Sordaria macrospora. Autophagy 9: 33-49.

Walz, M. \& Kück, U., (1995) Transformation of Sordaria macrospora to hygromycin B resistance: characterization of transformants by electrophoretic karyotyping and tetrad analysis. Current Genetics 29: 88-95.

Wang, C.-L., Shim, W.-B. \& Shaw, B.D., (2010) Aspergillus nidulans striatin (StrA) mediates sexual development and localizes to the endoplasmic reticulum. Fungal Genetics and Biology 47: 789-799.

Wang, C.-L., Shim, W.-B. \& Shaw, B.D., (2016) The Colletotrichum graminicola striatin orthologue Str1 is necessary for anastomosis and is a virulence factor. Molecular Plant Pathology 17: 931-942.

Wang, S.S., Esplin, E.D., Li, J.L., Huang, L., Gazdar, A., Minna, J. \& Evans, G.A., (1998) Alterations of the PPP2R1B gene in human lung and colon cancer. Science 282: 284-287.

Watson, M.L., (1955) The nuclear envelope: Its structure and relation to cytoplasmic membranes. The Journal of Biophysical and Biochemical Cytology 1: 257-270.

Wedlich-Söldner, R., Schulz, I., Straube, A. \& Steinberg, G., (2002) Dynein supports motility of endoplasmic reticulum in the fungus Ustilago maydis. Molecular Biology of the Cell 13: 965-977.

Wera, S., Fernandez, A., Lamb, N.J.C., Turowski, P., Hemmings-Mieszczak, M., Mayer-Jaekel, R.E. \& Hemmings, B.A., (1995) Deregulation of translational control of the $65-\mathrm{kDa}$ regulatory subunit (PR65 $\alpha$ ) of protein phosphatase 2A leads to multinucleated cells. Journal of Biological Chemistry 270: 21374-21381.

Werner, A., (2017) The interplay of SmNBR1 and SmATG8 in selective autophagy of the filamentous fungus Sordaria macrospora. Genetics of Eukaryotic Microorganisms: 1-171.

Werner, A., Herzog, B., Frey, S. \& Pöggeler, S., (2016) Autophagy-associated protein SmATG12 is required for fruiting-body formation in the filamentous ascomycete Sordaria macrospora. PLOS ONE 11: e0157960. 


\section{References}

Whiteway, M., Hougan, L., Dignard, D., Thomas, D.Y., Bell, L., Saari, G.C., Grant, F.J., O'Hara, P. \& MacKay, V.L., (1989) The STE4 and STE18 genes of yeast encode potential $\beta$ and $\gamma$ subunits of the mating factor receptor-coupled G protein. Cell 56: 467-477.

Wielowieyski, P.A., Sevinc, S., Guzzo, R., Salih, M., Wigle, J.T. \& Tuana, B.S., (2000) Alternative splicing, expression, and genomic structure of the $3^{\prime}$ region of the gene encoding the sarcolemmalassociated proteins (SLAPS) defines a novel class of coiled-coil tail-anchored membrane proteins. Journal of Biological Chemistry 275: 38474-38481.

Wigle, J.T., Demchyshyn, L., Pratt, M.A.C., Staines, W.A., Salih, M. \& Tuana, B.S., (1997) Molecular cloning, expression, and chromosomal assignment of sarcolemmal-associated proteins: A family of acidic amphipathic $\alpha$-helical proteins associated with the membrane Journal of Biological Chemistry 272: 32384-32394.

Wilson, C.M., (1979) Studies and critique of Amido Black 10B, Coomassie Blue R, and Fast Green FCF as stains for proteins after polyacrylamide gel electrophoresis. Anal Biochem 96: 263-278.

Witkos, T.M. \& Lowe, M., (2015) The Golgin family of coiled-coil tethering proteins. Frontiers in Cell and Developmental Biology 3: 86.

Wu, H. \& Parsons, J.T., (1993) Cortactin, an 80/85-kilodalton pp60src substrate, is a filamentous actinbinding protein enriched in the cell cortex. The Journal of Cell Biology 120: 1417-1426.

Wullschleger, S., Loewith, R., Oppliger, W. \& Hall, M.N., (2005) Molecular organization of target of rapamycin complex 2. Journal of Biological Chemistry 280: 30697-30704.

Wurzenberger, C. \& Gerlich, D.W., (2011) Phosphatases: providing safe passage through mitotic exit. Nat Rev Mol Cell Biol 12: 469-482.

Xiang, Q., Rasmussen, C. \& Glass, N.L., (2002) The ham-2 locus, encoding a putative transmembrane protein, is required for hyphal fusion in Neurospora crassa. Genetics 160: 169-180.

Yu, Y.B., (2002) Coiled-coils: stability, specificity, and drug delivery potential. Advanced Drug Delivery Reviews 54: 1113-1129.

Zhang, H., Chen, J., Wang, Y., Peng, L., Dong, X., Lu, Y., Keating, A.E. \& Jiang, T., (2009) A computationally guided protein-interaction screen uncovers coiled-coil interactions involved in vesicular trafficking. Journal of Molecular Biology 392: 228-241.

Zhang, H., Ma, X., Deng, X., Chen, Y., Mo, X., Zhang, Y., Zhao, H. \& Ma, D., (2012) PDCD10 interacts with STK25 to accelerate cell apoptosis under oxidative stress. Frontiers in Bioscience 17: 2295-2305.

Zhang, H., Ma, X., Peng, S., Nan, X. \& Zhao, H., (2014) Differential expression of MST4, STK25 and PDCD10 between benign prostatic hyperplasia and prostate cancer. International Journal of Clinical and Experimental Pathology 7: 8105-8111.

Zhang, H., Mukherjee, M., Kim, J.-E., Yu, W. \& Shim, W.-B., (2017) Fsr1, a striatin homolog, forms an endomembrane-associated complex that regulates virulence in the maize pathogen Fusarium verticillioides. Molecular Plant Pathology 3: 1-15.

Zhao, B., Tumaneng, K. \& Guan, K.-L., (2011) The Hippo pathway in organ size control, tissue regeneration and stem cell self-renewal. Nat Cell Biol 13: 877-883.

Zhou, Z., Munteanu, E.L., He, J., Ursell, T., Bathe, M., Huang, K.C. \& Chang, F., (2015) The contractile ring coordinates curvature-dependent septum assembly during fission yeast cytokinesis. Molecular Biology of the Cell 26: 78-90. 


\section{Supplement}

A

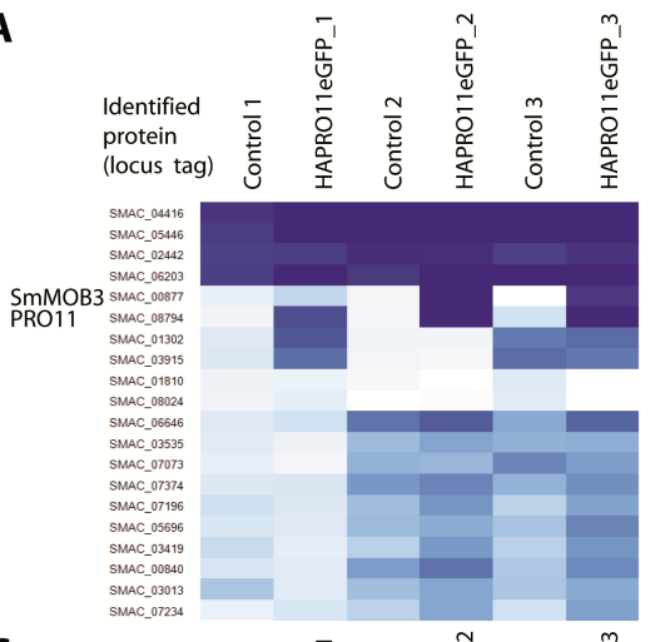

C

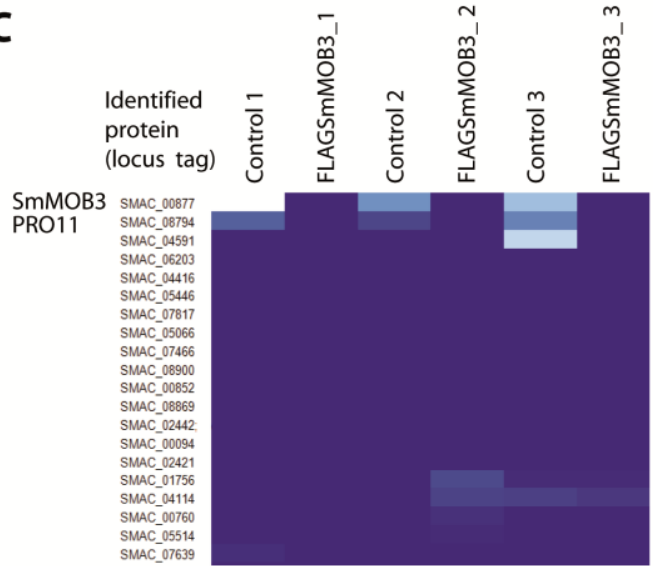

$\mathbf{E}$

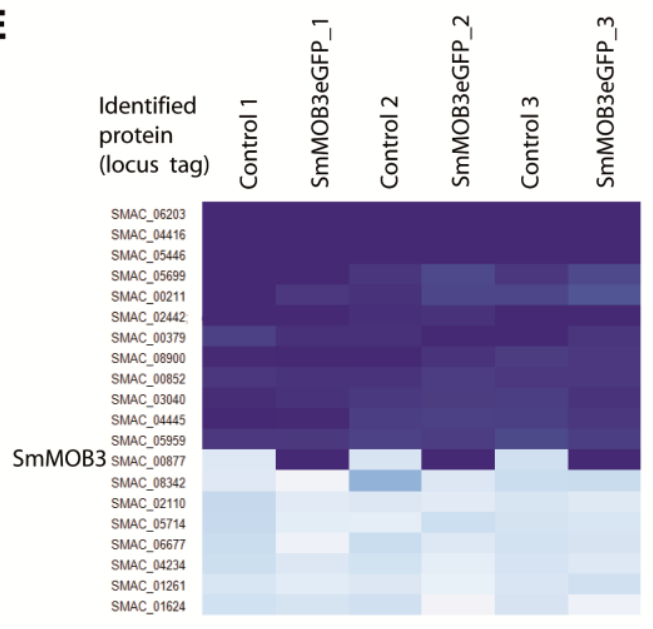

B
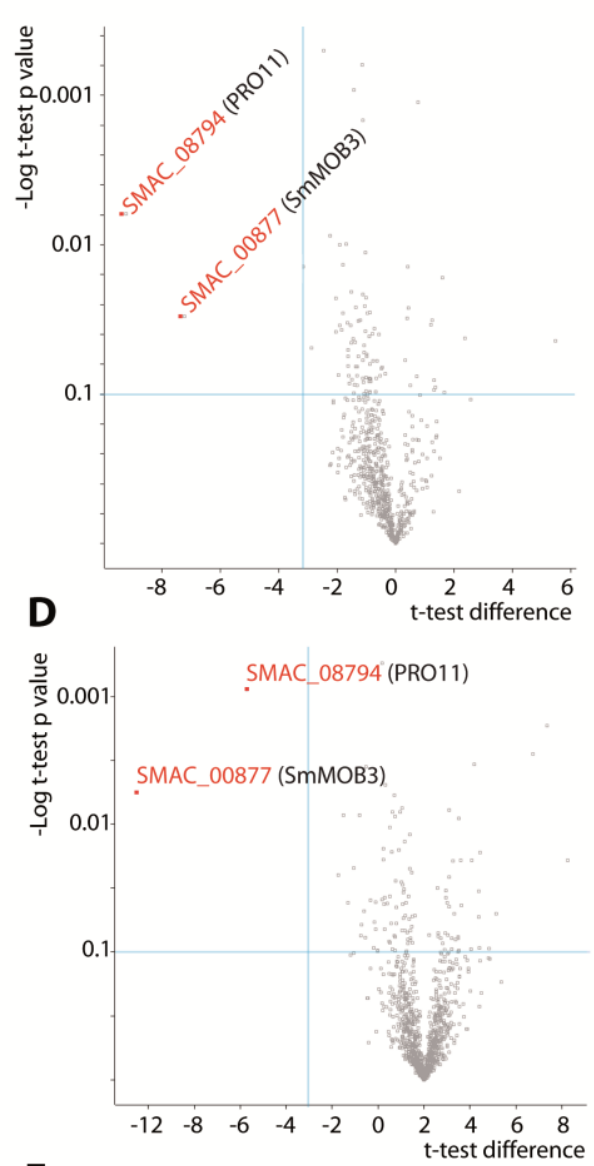

$\mathbf{F}$

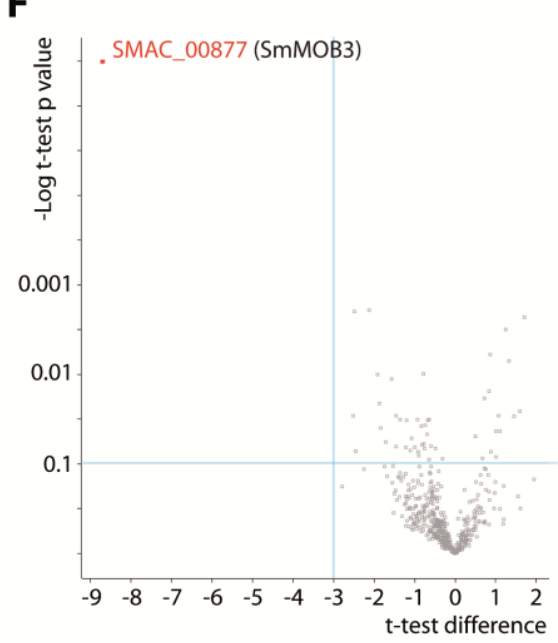

Figure S 1: LC/MS results of HAPRO11eGFP, FLAGSmMOB3 and SmMOB3eGFP pull-downs represented as heat maps and scatter plots

Each pull-down and LC/MS A/B: HAPRO11eGFP, C/D: FLAGSmMOB3 and E/F: SmMOB3eGFP was performed in three biological replicates. For simplicity, the bait proteins and following 20 proteins identified are presented. The intensity scale between 16 and 28 is presented. The scatter plots show proteins calculated to be significant using the t-test (t-test difference $<-3$ and $p$-value $<0.1$ ). Significant proteins are labeled in red and the locus tag is given. 
Supplement

A

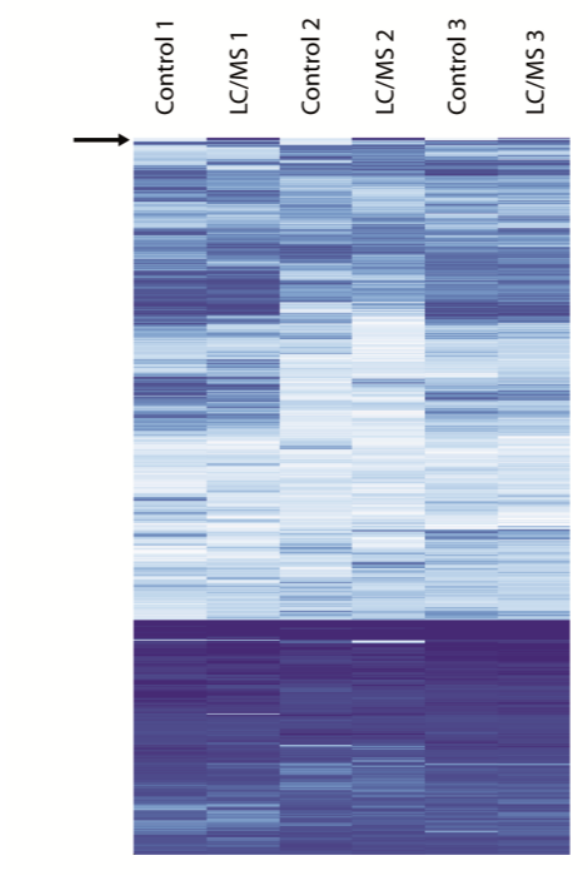

C

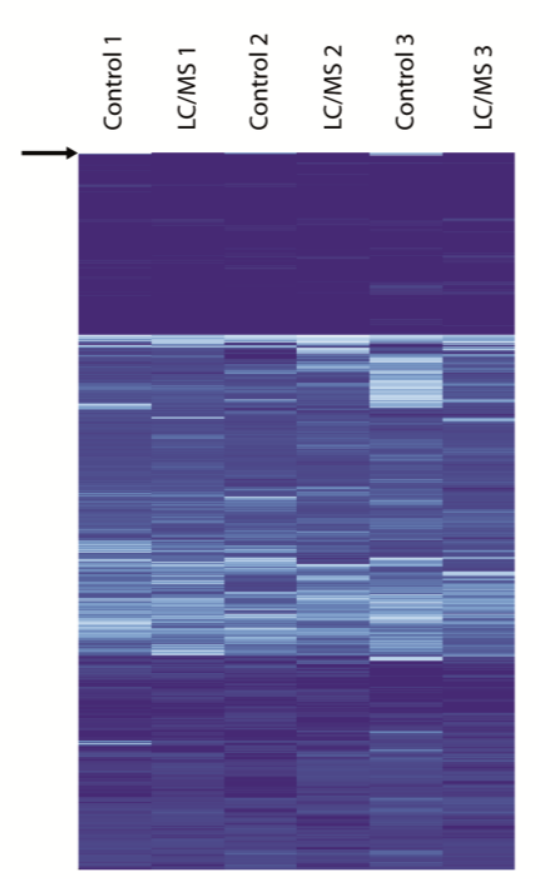

B

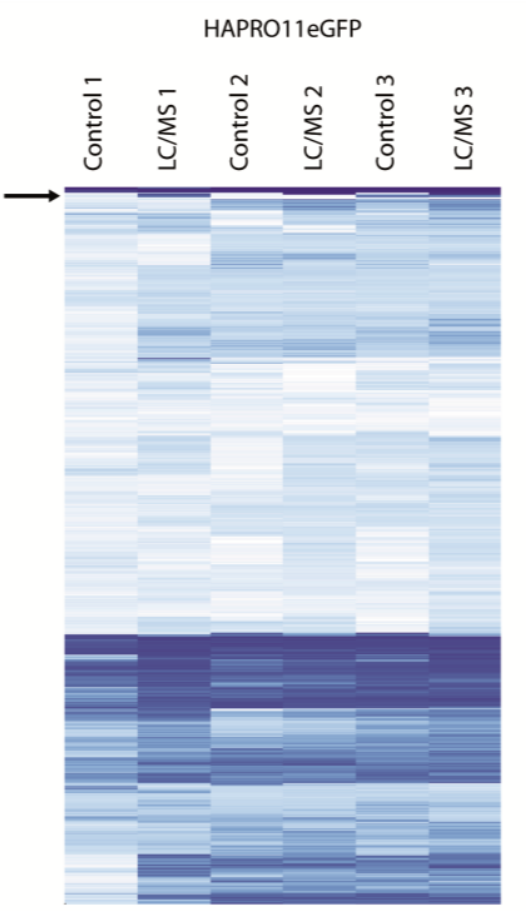

D

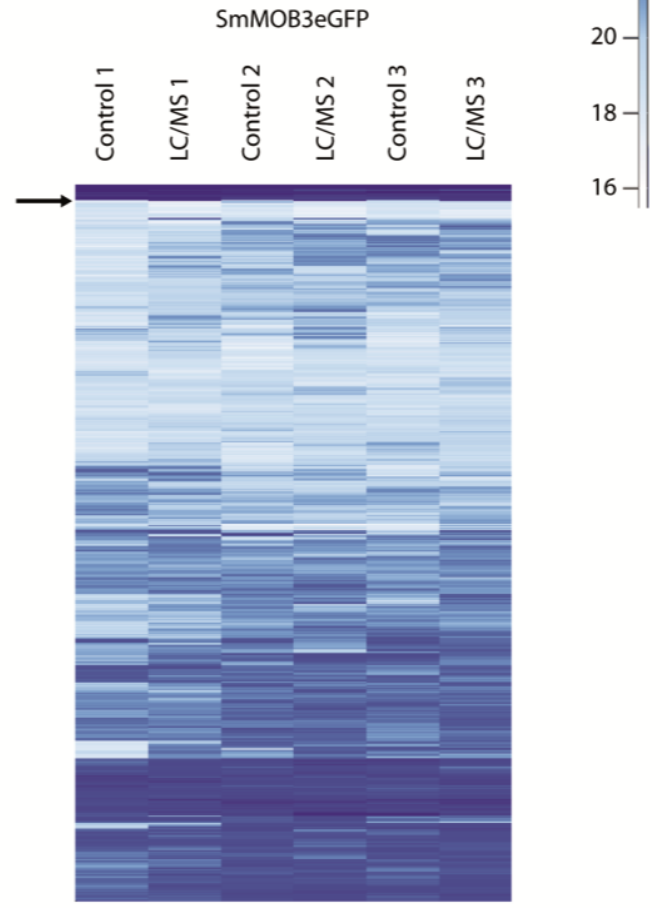

Figure S 2: Heat maps of all proteins identified in LC/MS in 4 different pull-downs

Each pull-down and LC/MS A: HAPRO11, B: HAPRO11eGFP, C: FLAGSmMOB3 and D: SmMOB3eGFP was performed in three biological replicates and the complete heat maps are given. Bait proteins are indicated with an arrow and the intensity scale between 16 and 28 is presented. 


\section{Supplement}

Sm_SCI1 (298 AA) Nc SCI1 (292 AA)

Pa_SCI1 (400 AA)

Ct SCl1 (385 AA)

Vd SCI1 (407 AA)

Mo SCI1 (308 AA)

Af_SCI1 (390 AA)

An_SCI1 (444 AA)

Hs SIKE (207 AA)

Mm_SIKE (207 AA)

Gg_SIKE (216 AA)

XI_SIKE (205 AA)

Dr-SIKE (211 AA)

Hs_1FRFR1OP2 (253 AA)

Mm_1FGFR1OP2 (253 AA)

Hs 2FRFR1OP2 (215 AA)

Mm 2FRFR1OP2 (215 AA)

Gg_FRFR1OP2 (217 AA)

XI_FRFR1OP2 (215 AA)

Dr_FRFR1OP2 (215 AA)

Dm_FGOP2 (315 AA)

Sc_Far3 (204 AA)

Sp Csc4 (166 AA)

Sc_Far7 (221 AA)

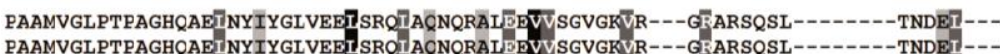
PAAMVGLPTPAGHQAEINYIYGLVEEISRQIACNQRALEBVVSGVGKVR---GFARSQSL--------TNDEI-QGAMVGVPIPAGQA SEI TFIYNMVDTI SGQI ACNQRTL GGGMA--PIPAGHQAEINYIYGIVEEISRQIAENQRAM DMVEIVGKVR---NKALEKEL-----

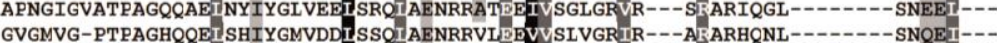

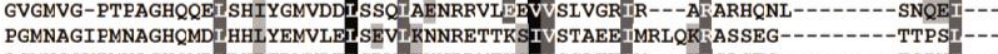
AGMNGGVPMNAGHQMDIRHLFDMVLEISDVLKNNRDMTKS IVSSAEEIM---KRSASEG---------TSPNI------------MSCTIEKILTDAKTLLERIREHDAAAE SLVDQSAALH---RRVAAMREAGTALPDOYOEDA---

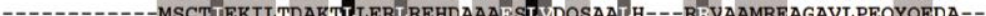
------MSCSIEKVL GDARTLLERLKEHDTAAESLIEQSSVIG---QKIHSMKEVGNTLPDKYMEEN--------MSCTIERAIADARAIVERLRDHDDAAESLIEQTTAIS---KRVEAMKQ-------YQEEI---

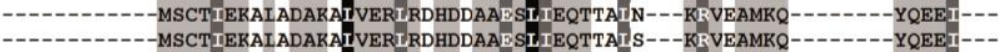
------MSCT IERAL ADARAIVERLREHDNAAD ALIEQTTAIN---KRVEAMKQ------YQEEI---------MSCTIEKVI ADAKEIVERLREHD GAA SLIEQTTTIN---KRVEAMKQ------ YQEEV-----------SNLSVGQIIMDAQR ASRVKDLDALGTALLEEAENNN---RLVESLRQ-------YQDDI--------MNSGGTDSFDYLLLTTKAISAECRANRQETDR IELLLKRIAK-QSGISYDNL-------SKNIIPDS

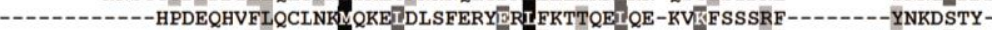

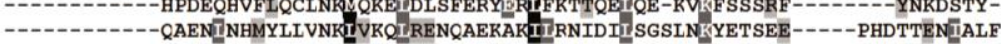

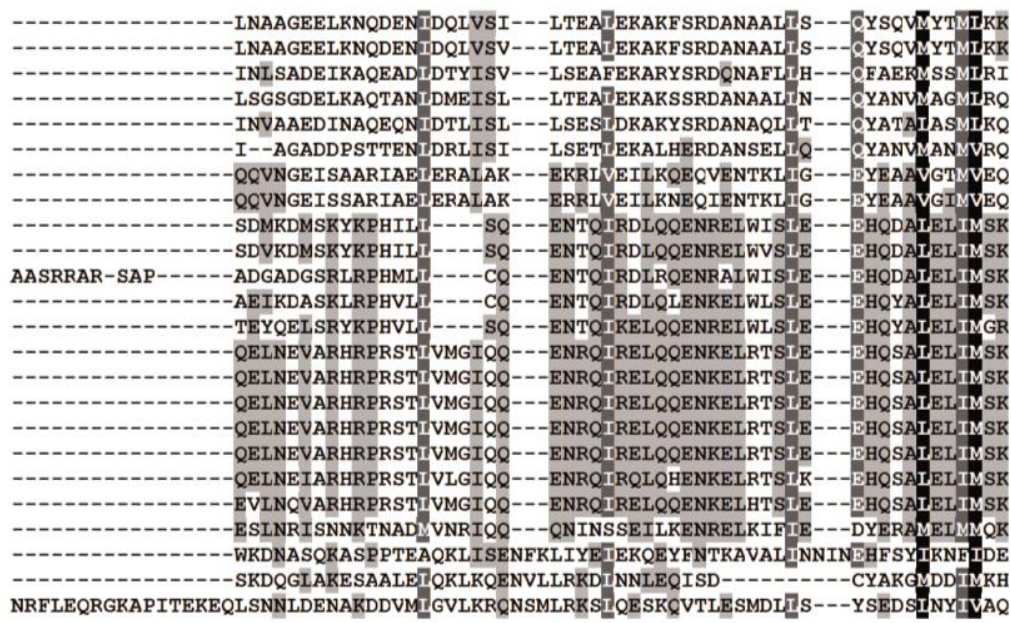

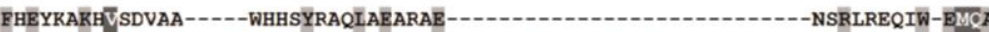

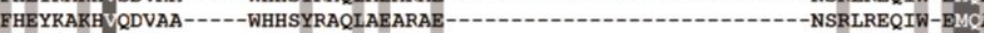
FHEYKAKHVSDVST-----WHKSYRKQL ADARAE-FHEYKARHVADVAA-----WHRSYRAQLAEARAE---FHEYKAKHVADVSS-----WHRSYRSQIAEARAE---1-10

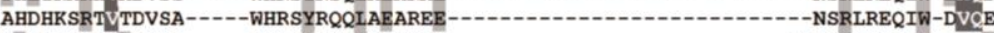

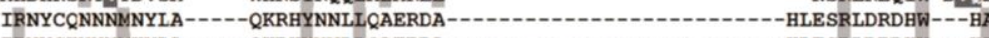
IRNYCHNNNMHYLA-----QRRHYNNLLQAERDA--------------------HLESRLDRDHW---HA YRKCMLQLZVAKRA -----VDAEPVLRAHQSHSA---

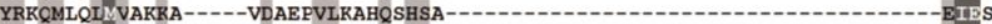

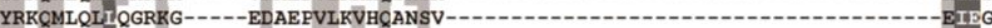
YRKCQMLQLIANKIP-----APTEPVLEAHKTFSS-----1

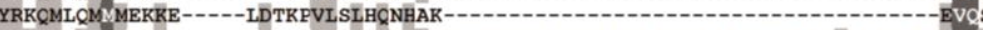
YRECMFRLIMASKK-----DD PGI IMKLKEQHSKIDMVHRNKSEGFFLDASRHILEAPQHGLERRHLEANQNELC YRECMFRLIMASKK------DDPGI IMKLKEQHSKIDMVHRN SCEGFFLDASRHILEAPQHGLERRHLEANQNDLC YRECMFRLIMASKK-----DDPGI IMKLKEQHSK----

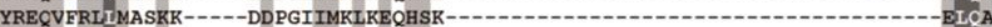

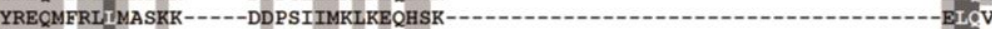

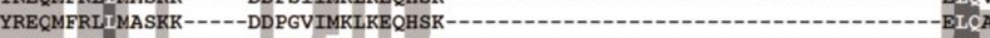
YREQVFRLIMASKK-----ED PTIVTQLREQHTN--1-1-10

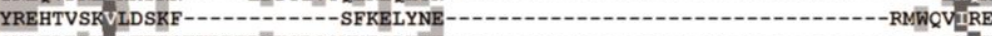

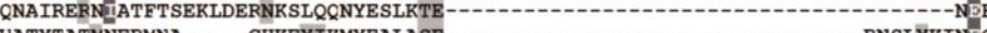
VATYTATMNERMNA-----CHKEYIKMYEALASE-LRGNILMHHKETIK--L--IRQKFQTETIPLEDE-- 


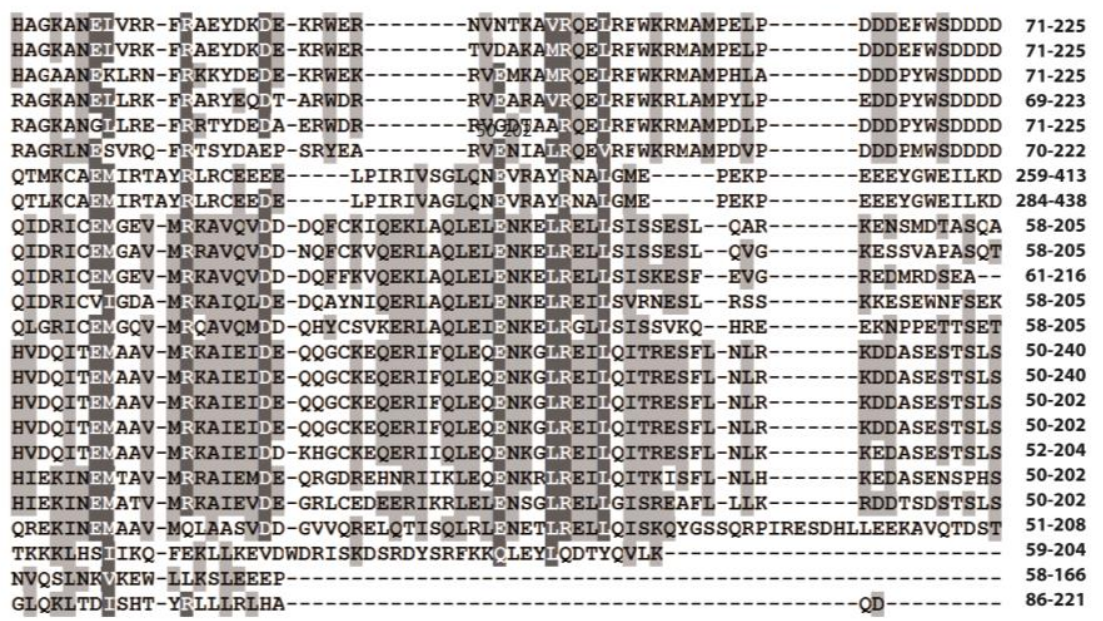

Figure S 3: Alignment of small coiled-coil orthologs associated with the STRIPAK found in animals, fungi and yeasts

Orthologs were identified with BLASTP search using amino acid sequences of human SIKE and FGFR1OP2 and S. macrospora SCl1. The multiple sequence alignment was performed with MAFFT version 7.182 (Katoh \& Standley, 2013) using the amino-acid sequences: Sm SCI1, S. macrospora (XP_003349276.1); Nc SCI, $N$. crassa (XP_960970.1); Ct SCl, Chaetomium thermophilum (XP_006696634.1); Vd SCI, Verticillium dahliae (EGY17808.1); $\mathrm{Pa} \mathrm{SCl}$, Podospora anserina (XP_001912083.1); $\mathrm{Mo} \mathrm{SCl}$, Magnaporthe oryzae (XP_003715088.1); Af SCl, Aspergillus fumigatus (XP_752657.2); An SCl, A. nidulans (XP_658614.1); Sc, S. cerevisiae Far3 (AAB39035) and Far7 (P43592); Sc Csc4, S. pombe Csc4 (O14043); Dm FGOP2, D. melanogaster FGOP2 (NP_609084); Hs SIKE, H. sapiens SIKE (NP_079349.2); Mm SIKE, M. musculus SIKE (NP_079955.1); Gg SIKE, Gallus gallus domesticus SIKE (NP_001006324.2); XI SIKE, Xenopus laevis SIKE (NP_001085744.1); Dr SIKE, Danio rerio SIKE (XP_696578.2); Hs 1FGFR1OP2, H. sapiens isoform 1 FGFR1OP2 (NP_056448.1); Hs 2FGFR1OP2, H. sapiens isoform 2 FGFR1OP2 (NP_001165358.1); Mm 1FGFR1OP2, $M$. musculus isoform 1 FGFR1OP2 (NP_080494.1); Mm_2FGFR1OP2, M. musculus isoform 2 FGFR1OP2 (XP_006536173.1); Gg FGFR1OP2, G. domesticus FGFR1OP2 (NP_001007856.1); XI FGFR1OP2, X. laevis FGFR1OP2 (NP_001079401.1); Dr FGFR1OP2, D. rerio FGFR1OP2 (NP_956249.1).

The alignment is represented with GeneDoc (Nicholas \& Nicholas, 1997). The overall protein lengths in amino acids are presented in brackets. The amino-acid regions used for the alignment are indicated at the right hand side at the end of the alignment. Amino acids conserved in all sequences are shaded in black, amino acids conserved in 19-23 of 24 sequences a shaded in dark gray and amino acids conserved in 10-18 of 24 sequences are shaded in light gray. 
A

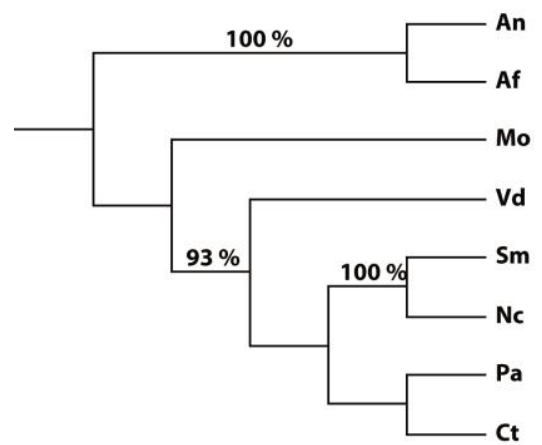

B

Sm Nc Ct Vd Pa Mo Af An

Sm $100 \%$

Nc $93 \% \quad 100 \%$

Ct $\quad 67 \% \quad 70 \% \quad 100 \%$

Vd $64 \% \quad 66 \% \quad 64 \% \quad 100 \%$

$\begin{array}{llllll}\text { Pa } & 63 \% & 65 \% & 62 \% & 63 \% & 100 \%\end{array}$

Mo $\quad 56 \% \quad 58 \% \quad 59 \% \quad 57 \% \quad 59 \% \quad 100 \%$

Af $\quad 20 \% \quad 20 \% \quad 22 \% \quad 21 \% \quad 18 \% \quad 20 \% \quad 100 \%$

An $\quad 18 \% \quad 19 \% \quad 21 \% \quad 22 \% \quad 17 \% \quad 20 \% \quad 85 \% \quad 100 \%$

Figure S 4: Neighbor-joining tree and pair-wise comparison of SCl1 amino-acid sequences in filamentous ascomycetes

Neighbor-joining tree $\mathbf{A}$ and pair wise comparison $\mathbf{B}$ of amino-acid sequences of $\mathrm{Sm}, \mathrm{S}$. macrospora (XP_003349276.1); Nc, N. crassa (XP_960970.1); Ct, C. thermophilum (XP_006696634.1); Vd, V. dahliae (EGY17808.1); Pa, P. anserina (XP_001912083.1); Mo, M. oryzae (XP_003715088.1); Af, A. fumigatus (XP_752657.2); An, A. nidulans (XP_658614.1) aligned with MAFFT (Katoh \& Standley, 2013). SCl1 orthologs of Sordariomycetes or Eurotiomycetes cluster together and the sequence identity is highest within a class.

ATG AAC GGC ATG CAT GCT GGA AAT ATG CCC GCG GCC ATG GTT GGC CTC CCT ACG CCG GCA $\begin{array}{llllllllllllllllllll}M & N & G & M & H & A & G & N & M & P & A & A & M & V & G & L & P & T & P & A\end{array}$

GGG CAC CAA GCC GAG CTA AAT TAC ATC TAC GGC TTG GTT GAG GAG TTG AGC CGA CAG CTT $\begin{array}{llllllllllllllllllll}G & H & Q & A & E & L & N & Y & I & Y & G & L & V & E & E & L & S & R & Q & L\end{array}$

GCG CAG AAT CAG CGC GCC CTG GAG GAG GTA GTC TCG GGC GTT GGC AAA GTG CGC GGG CGA $\begin{array}{llllllllllllllllllll}A & Q & N & Q & R & A & L & E & E & V & V & S & G & V & G & K & V & R & G & R\end{array}$

GCA AGG TCA CAG TCT CTG ACA AAT GAC GAA CTA CTC AAT GCC GCC GGG GAA GAA CTC AAG $\begin{array}{llllllllllllllllllll}A & R & S & Q & S & L & T & N & D & E & L & L & N & A & A & G & E & E & L & K\end{array}$

\section{A gt atg tet ctc ccg ccc tet cat cet ctc act tac acg ccc ata acc tta cac tta cta}

act cat ctg tat ctg cat ag AC CAA GAC GAA AAC ATA GAC CAA CTA GTC TCC ATC CTC ACG $\begin{array}{llllllllllllll}N & Q & D & E & N & I & D & Q & L & V & S & I & L & T\end{array}$

GAA GCT CTC GAA AAA GCC AAA TTC TCT CGC GAC GCC AAC GCC GCC CTT TTG TCT CAA TAC $\begin{array}{llllllllllllllllllll}E & A & L & E & K & A & K & F & S & R & D & A & N & A & A & L & L & S & Q & Y\end{array}$

TCG CAA GTC ATG TAC ACC ATG CTC AAG AAA TTC CAC GAG TAC AAA GCC AAG CAC GTG TCC $\begin{array}{llllllllllllllllllll}S & Q & V & M & Y & T & M & L & K & K & F & H & E & Y & K & A & K & H & V & S\end{array}$

GAC GTG GCC GCC TGG CAC CAC TCG TAC CGG GCC CAG CTC GCC GAG GCG CGC GCG GAG AAT

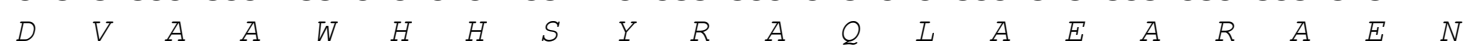

AGT CGG CTG CGC GAG CAG ATC TGG GAG ATG CAG GCG CAC GCG GGC AAA GCC AAC GAG CTG 
GTG CGG CGG TTC CGG GCC GAG TAC GAC AAG GAC GAA AAG CGC TGG GAG CGC AAT GTC AAT $\begin{array}{llllllllllllllllllll}V & R & R & F & R & A & E & Y & D & K & D & E & K & R & W & E & R & N & V & N\end{array}$

ACC AAG GCC GTG AGG CAG GAG CTG AGG TTC TGG AAA CGC ATG GCG ATG CCG GAA CTG CCA $\begin{array}{llllllllllllllllllll}T & K & A & V & R & Q & E & L & R & F & W & K & R & M & A & M & P & E & L & P\end{array}$ GAT GAT GAC GAG TTT TGG AGC GAT GAT GAT GAT GTT ATT GAT GGG GCG GAG AAG GAG AGG

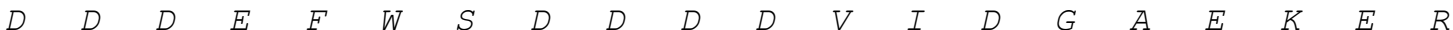

CAG AAG GAG ATA CAA AAG TTG GAT GCG GAG CAG CAG GCG CAG GCT GCG CAG GCT GTG GCG

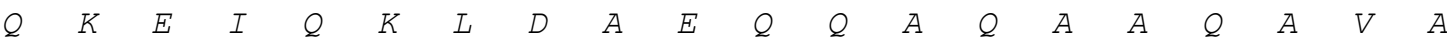

GAG GAG GCG GCT GCT AAT GAG GCT GCT GCT GAA AGT AGT GGT GGG TTG GGA GAG ATG AGT

$\begin{array}{llllllllllllllllllll}E & E & A & A & A & N & E & A & A & A & E & S & S & G & G & L & G & E & M & S\end{array}$

GGG AGT GGA AGT GGG AGT AAT GGT TCT GGC CTG ACG GGG GTA CCG CCG GAG GTA CCA CAG

CCG CCG GTT TCC

P P V S

Figure S 5: Genome sequence of sci1 and translated protein sequence

The genome sequence of sci1 with the corresponding translated protein sequence is presented. The intron at position $242-320$ is shown in gray.

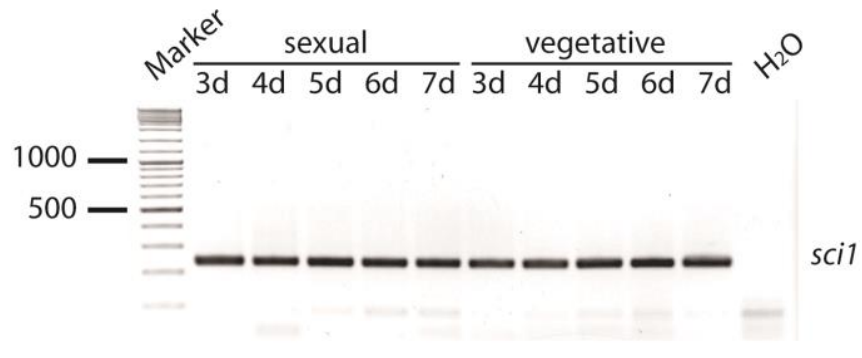

Figure S 6: sci1 fragment after quantitative reverse-transcription PCR

The products of the quantitative reverse-transcription PCR using the primer pair RTsci1f/RTsci1r were resolved on an $2 \%$ agarose gel. The sci1 fragment amplified from sexually and vegetatively grown S. macrospora wt had a size of $236 \mathrm{bp}$. The negative control supplemented with water is also presented. Marker: GeneRuler ${ }^{\mathrm{TM}}$ DNA Ladder Mix (Thermo Scientific). 


\section{Supplement}

A gDNA
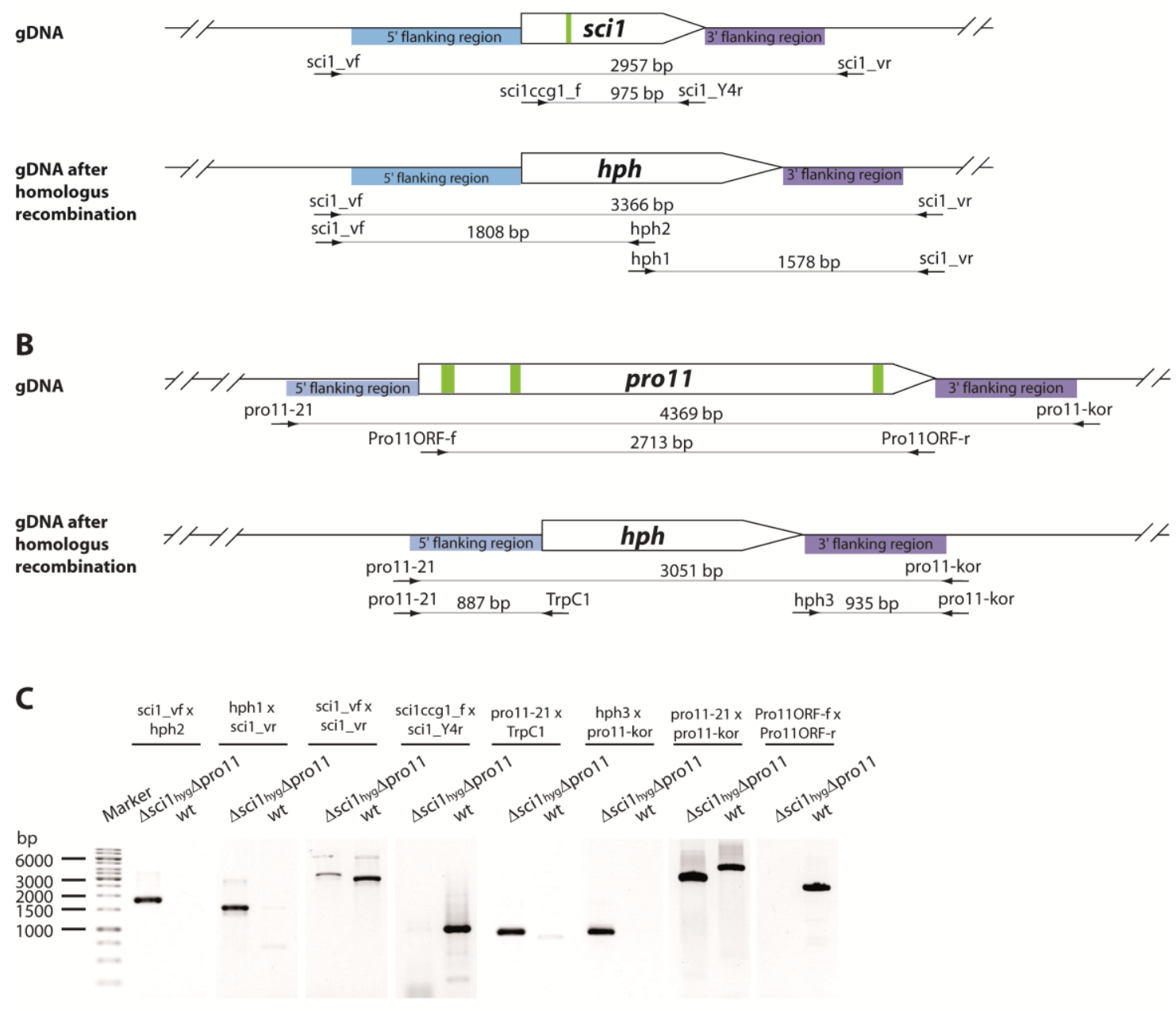

Figure S 7: Verification of the $\Delta \mathrm{sci}_{\mathrm{hyg}} \Delta$ pro11 strain by PCR

A: Schematic representation of the sci1 ORF and B: the pro11 ORF. Introns (green box), 5' flanking regions (blue) and 3' flanking regions (purple) are indicated. After homologous recombination the hph cassette replaced the whole sci1 or the whole pro11 ORF. Primer combinations and the corresponding fragment sizes are indicated. C: Verification of the $h p h$ cassette in the sci1 and the pro11 locus using PCR. Genomic DNA was isolated from wt and $\Delta$ sci $1_{\text {hyg }} \Delta$ pro11 and tested with indicated primer (black arrows) combinations. The marker GeneRuler ${ }^{\mathrm{TM}} 1$ kb DNA Ladder (Fisher Scientific) was used for gel-electrophoresis. 


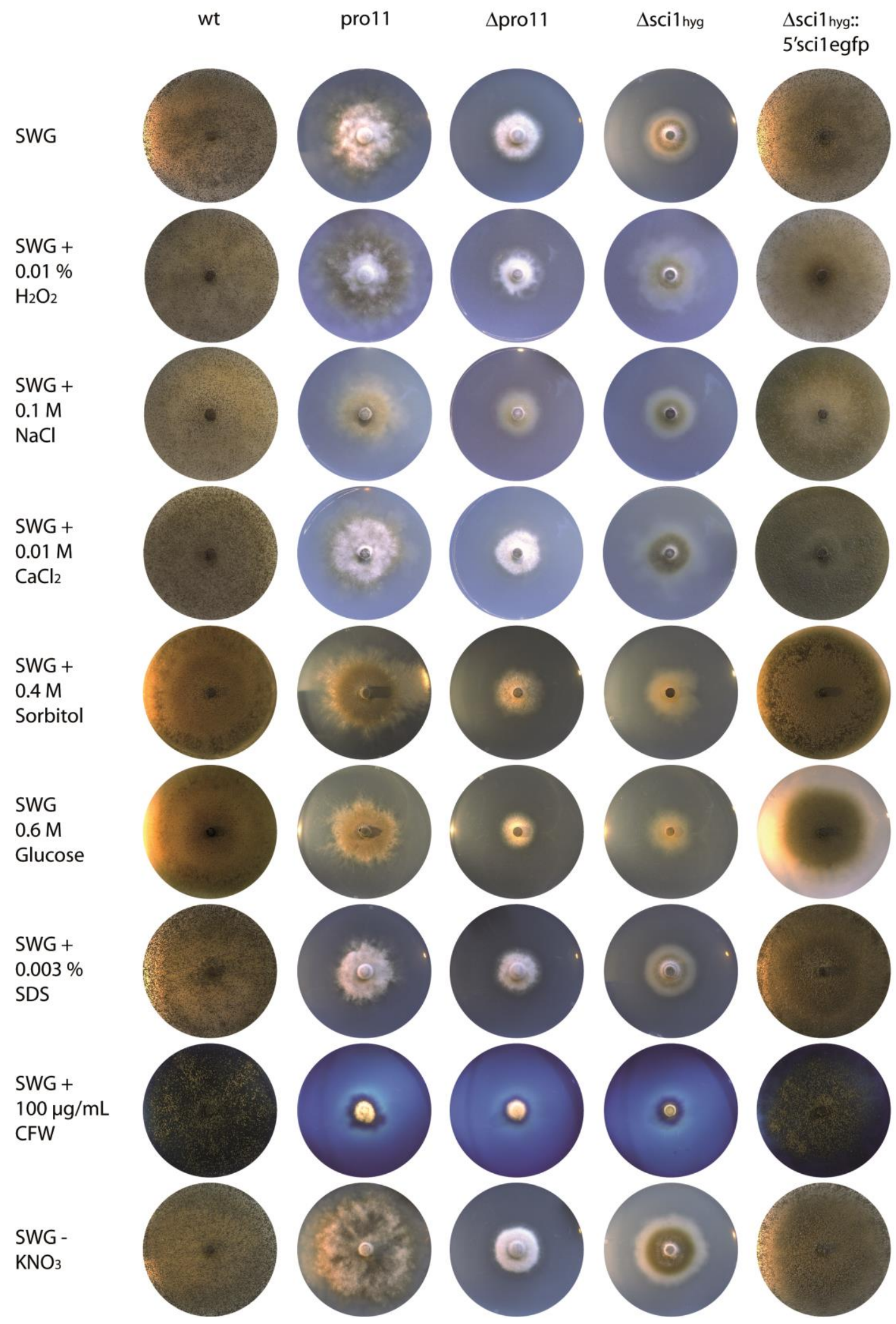

Figure S 8: Growth of wt, pro11, $\Delta$ pro11, $\Delta$ sci $1_{\text {hyg }}$ and $\Delta$ sci $1_{\text {hyg }}:: 5$ 'scilegfp under stress conditions Growth of strains wt, pro11, $\Delta$ pro11, $\Delta$ sci $1_{\text {hyg, }}$ and $\Delta$ sci $1_{\text {hyg }}: 5$ 'scilegfp on the indicated stress media for 7 days on petri dishes is presented. SWG was supplemented with $\mathrm{H}_{2} \mathrm{O}_{2}$ (oxidative stress), $\mathrm{NaCl}, \mathrm{CaCl}_{2}$ sorbitol and glucose (osmotic stress), SDS (cell wall stress), calcofluor white (CFW) (cell wall stress/cell wall integrity pathway) or prepared without $\mathrm{KNO}_{3}$ (nitrogen starvation) as indicated. 


\section{Supplement}
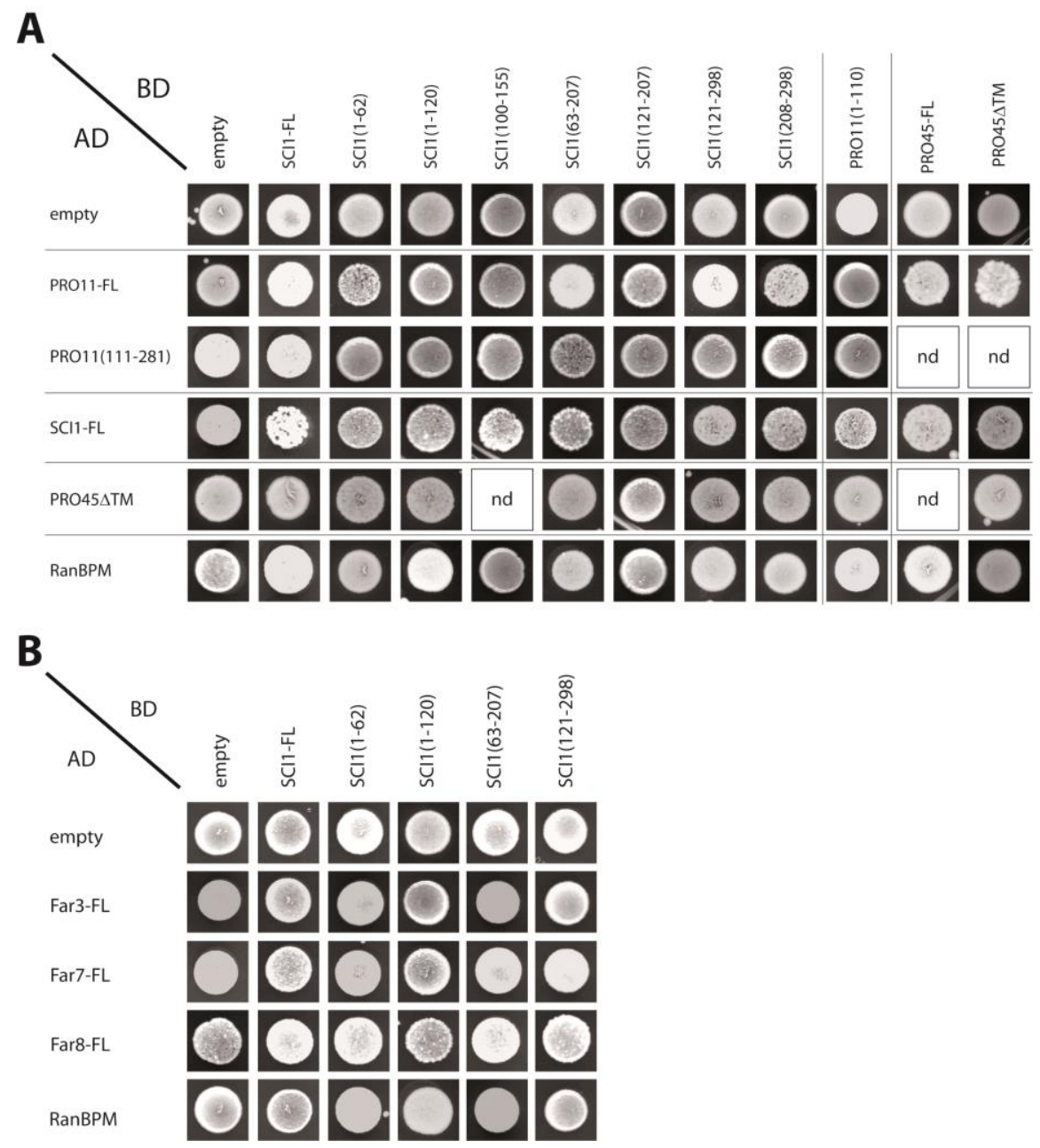

Figure S 9: $\mathrm{Y2H}$ growth control of full length and fragments of PRO11, SCI1 and full length Far3, Far7 and Far8

Indicated constructs fused to the Gal4-activating domain or binding-domain sequence were cotransformed into PJ69-4A and $20 \mu \mathrm{L}$ of each strain with an $\mathrm{OD}_{600} 0.1$ was dropped onto selective medium. The growth control show the respective combinations on synthetic defined medium without leucine and tryptophan (SD-LT). A: growth controls for interaction studies of SCI1, PRO11 and PRO45 including fragments of SCI1, PRO11 and PRO45. B: growth control for interaction studies of Far3, Far7 and Far8 with SCI1 and SCI1 fragments. 
Supplement

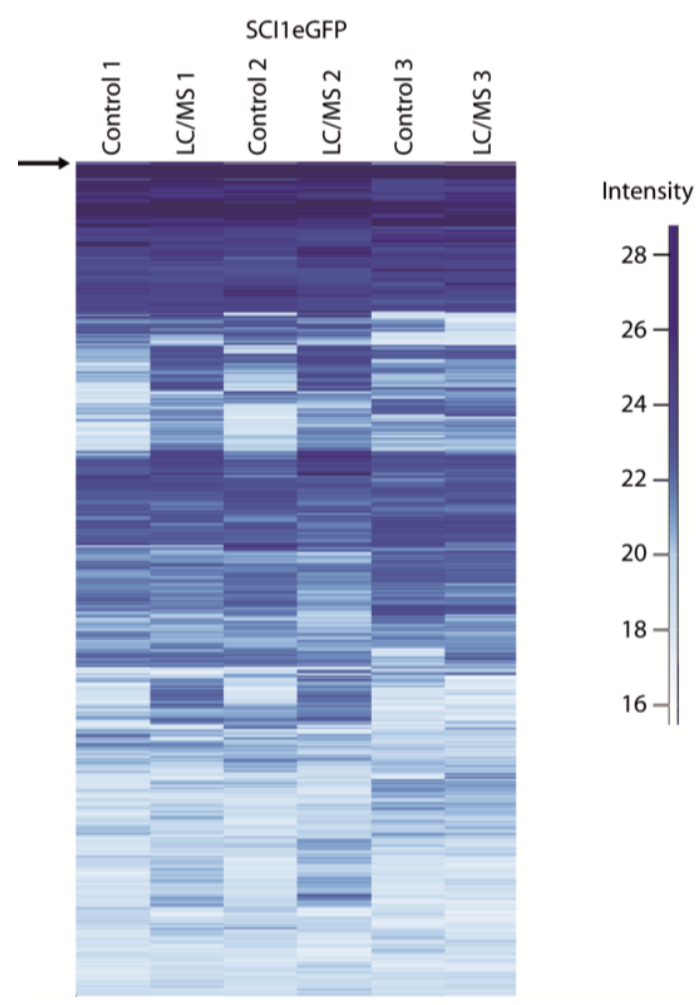

Figure S 10: Heat maps of all proteins identified in LC/MS in three SCI1eGFP pull-downs SCl1eGFP pull-downs were performed in three biological replicates and the complete heat map is given. The bait protein SCI1eGFP is indicated with an arrow and the intensity scale between 16 and 28 is presented. 


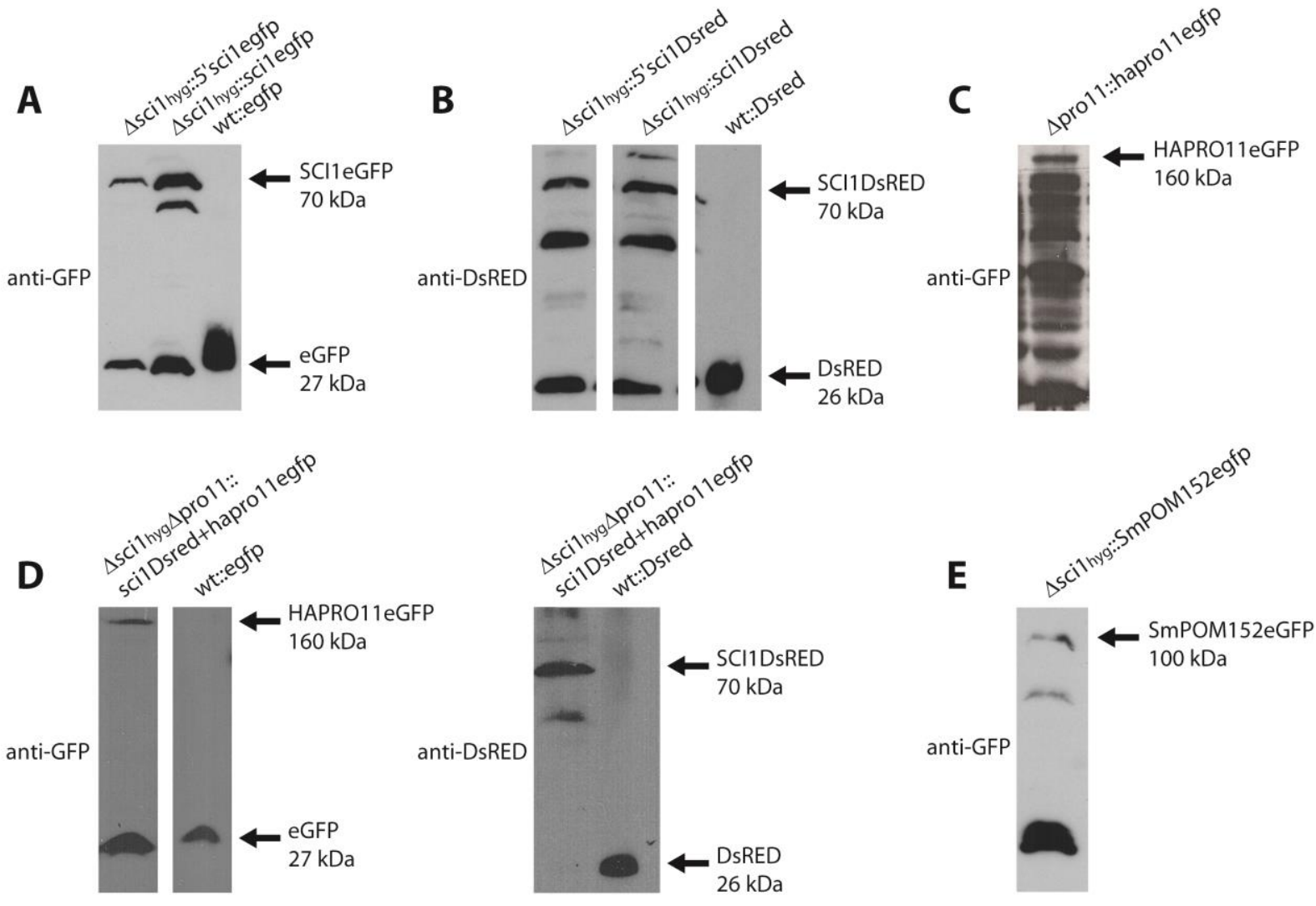

Figure S 11: Western blot using anti-GFP and anti-DsRED for detection of fusion proteins expressed in S. macrospora

Synthesis of A: SCl1eGFP, B: SCl1DsRED, C: HAPRO11eGFP, D: SCI1DsRED and HAPRO11eGFP and E: SmPOM152eGFP fusion proteins (black arrow) in the respective strains was successfully shown by Western blot experiments. The sci1 gene fused to a egfp or Dsred was either under the control of the endogenous sci1 promoter (5'scilegfp) or under the control of the promoter of the clock controlled gene 1 of $N$. crassa (scilegfp). Also the hapro11egfp fusion gene was under the control of the promoter of the clock controlled gene 1 of $N$. crassa. Proteins isolated from indicated strains were detected with anti-GFP or anti-DsRED. The wt::egfp and wt::Dsred strains served as controls. The apparent molecular weight of the fusion proteins, eGFP and DsRED is indicated in $\mathrm{kDa}$. 
$\Delta$ sci1hyg $\Delta$ pro11::sci1Dsred+hapro11egfp
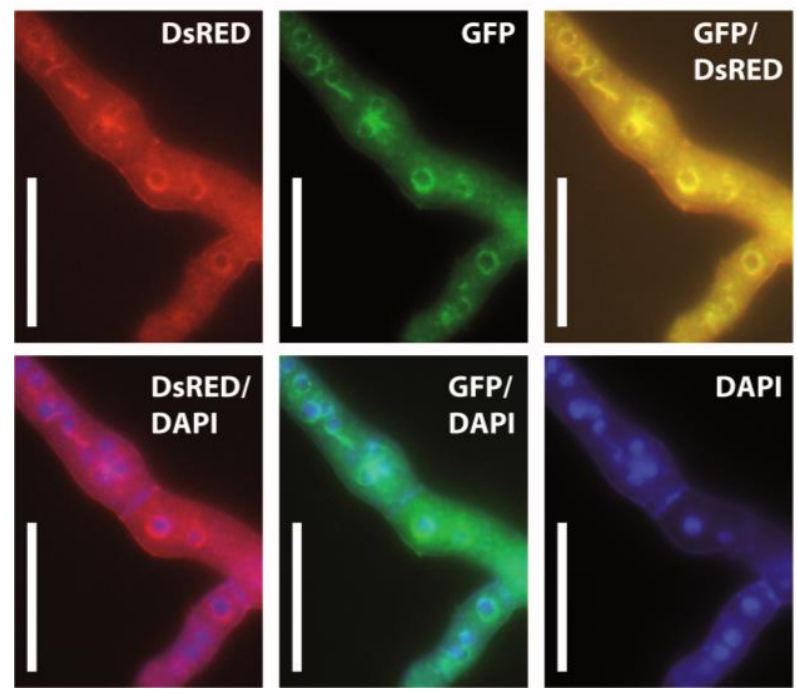

Figure S 12: Colocalization of SCI1DsRED and HAPRO11eGFP and visualization of nuclei

SCI1DsRED and HAPRO11eGFP colocalized in roundish structures around nuclei stained with DAPI. Both fusion proteins also localized to thread like structures and punctiform structures in the cytoplasm. Scale bar: $20 \mu \mathrm{m}$.

A

wt::

h2btdTomato

$\Delta$ sci1hyg::

h2btdTomato

B

wt::

mitoDsred

$\Delta$ sci1hyg:: mitoDsred

C

wt::

DsredKDEL

$\Delta$ sci1hyg:: DsredKDEL
DIC
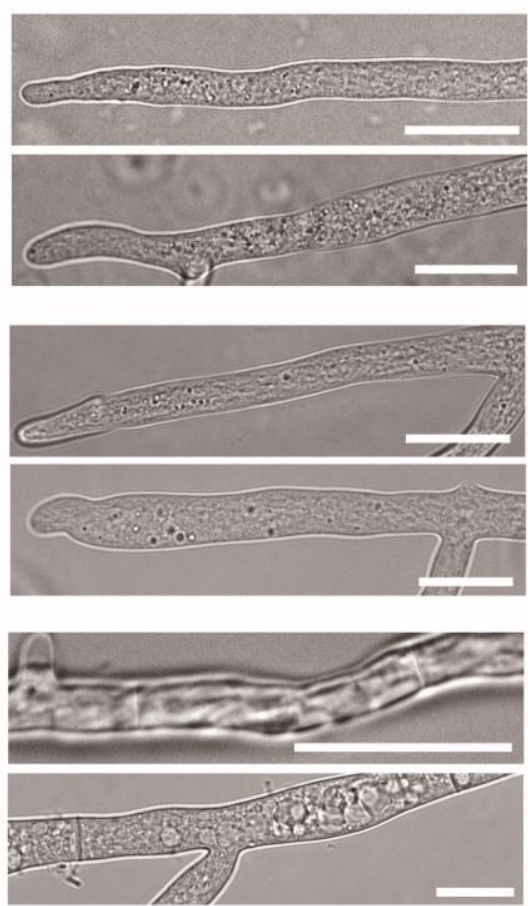

TdTomato/ DsRED
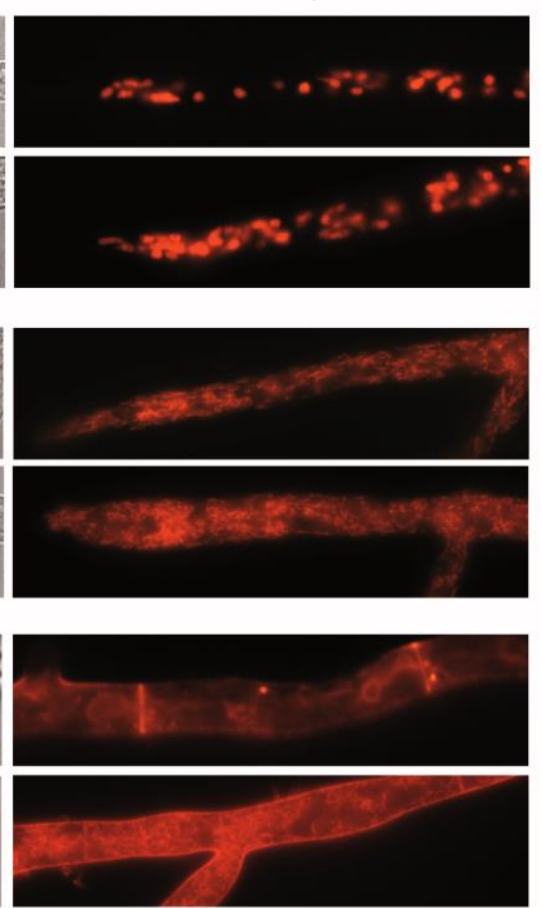

Figure S 13: Nuclei, mitochondria and the ER in wt and $\Delta$ sci1

A: Nuclei, B: Mitochondria, and C: Endoplasmic reticulum (ER) were visualized in vegetative hyphae of S. macrospora using indicated marker proteins tagged with red fluorescent protein DsRED or TdTomato. Scale bar size: $20 \mu \mathrm{m}$ 


\section{Supplement}

A
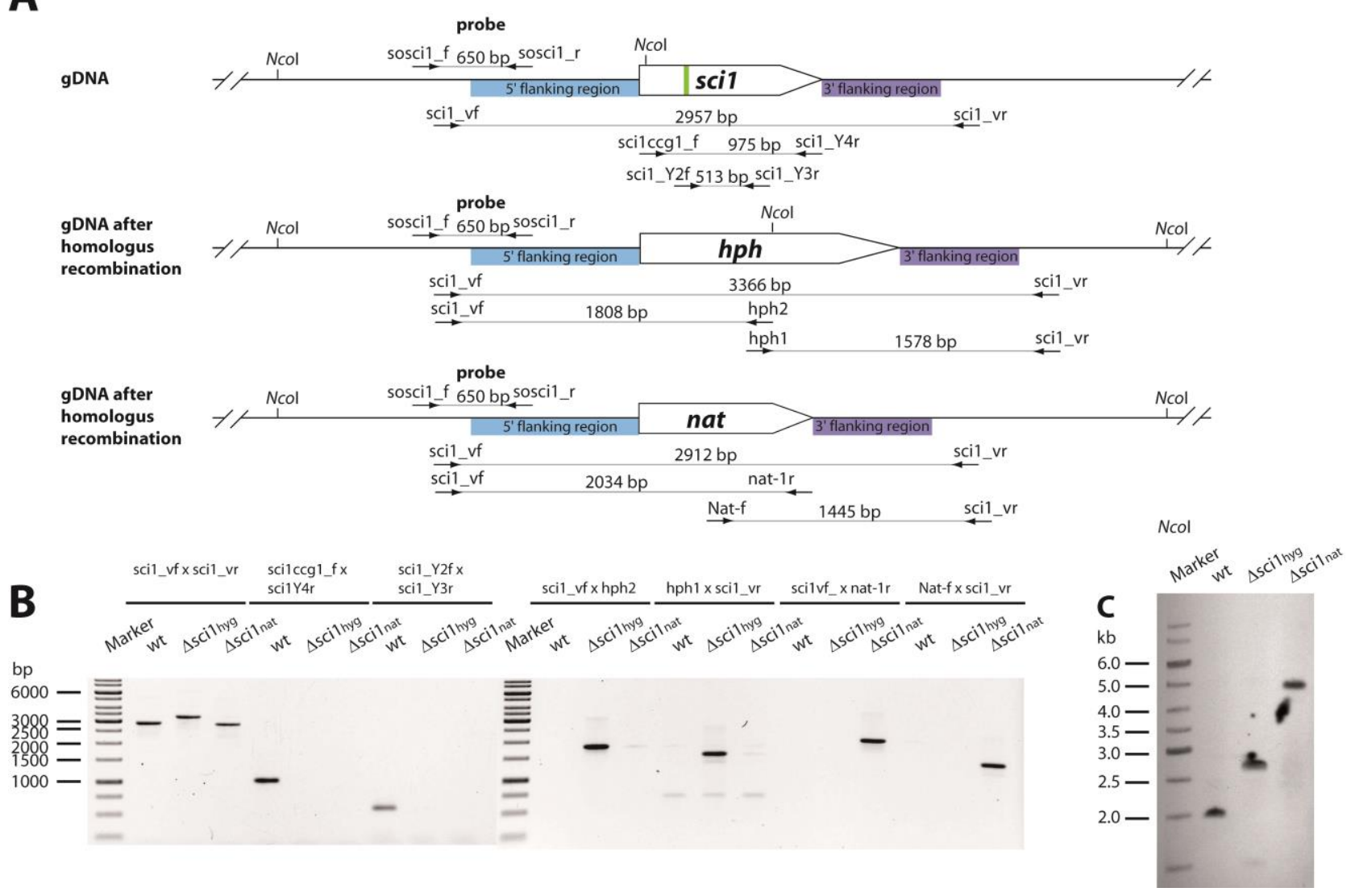

Figure S 14: PCR and Southern blot analysis of wt, $\Delta$ sci $1_{\text {hyg }}$ and, $\Delta s c i 1_{\text {nat }}$

A: Schematic representation of the scil ORF with one intron (green box), 5' flanking region (blue) and 3' flanking region (purple). After homologous recombination the resistance cassettes (either nat or hph) replaced the whole sci1 ORF. Primer combinations and the corresponding fragment sizes are indicated. The probe size and hybridization region is also indicated. B: Verification of homologous integration of the $h p h$ or the nat cassette into the sci1 locus using PCR. Genomic DNA was isolated from wt, $\Delta s c i 1_{\text {hyg }}$ and $\Delta s c i 1_{\text {nat }}$ strains and tested with indicated primer combinations. The marker, GeneRuler ${ }^{\mathrm{TM}} 1 \mathrm{~kb}$ DNA Ladder (Fisher Scientific) was used for both gel-electrophoresis and Southern blot. The fragments are given in bp for identification of the amplicons or in $\mathrm{kb}$ for the Southern blot. C: Confirmation of the $\Delta s c i 1_{\text {hyg }}$ and $\Delta s c i 1_{\text {nat }}$ strain was done by Soutern blot. The isolated gDNA was hydrolyzed with Ncol. The probe and the marker were labeled with alkaline phosphatase and signals were detected on X-ray films. Signals detected correspond to a fragment size of $2.0 \mathrm{~kb}$ for wt, $2.6 \mathrm{~kb}$ for $\Delta s c i 1_{\text {hyg }}$ and $4.8 \mathrm{~kb}$ for $\Delta \mathrm{sci} 1_{\text {nat }}$. 


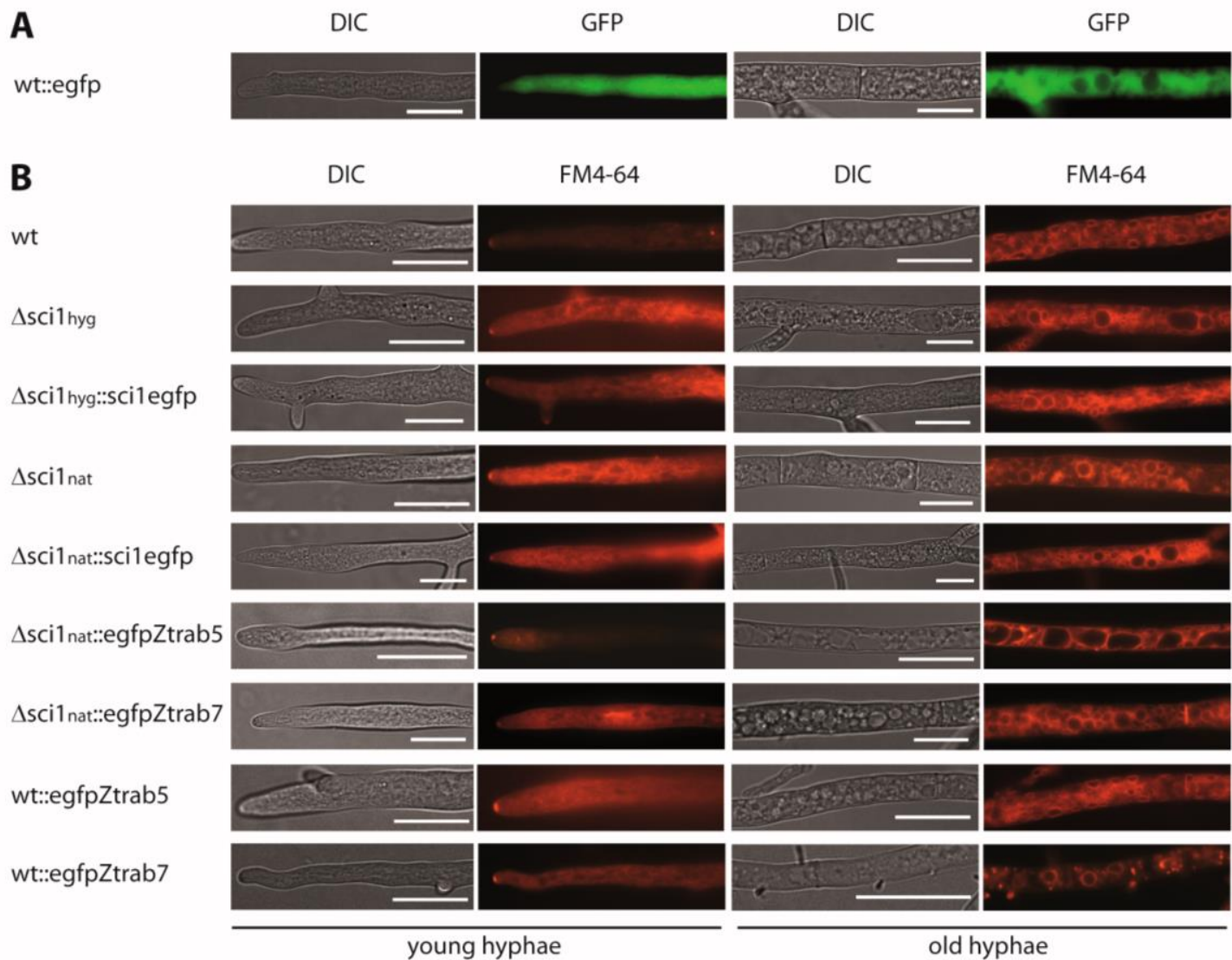

Figure S 15: Fluorescence microscopy of the wt::egfp control strain and strains stained with FM4-64 A: Signal of free eGFP in hyphae of the control strain wt::egfp. B: The indicated strains were stained with the red fluorescence dye FM4-64. FM4-64 entered through the hyphal tip and was localized to membranecontaining structures in old hyphae. Scale bar: $20 \mu \mathrm{m}$

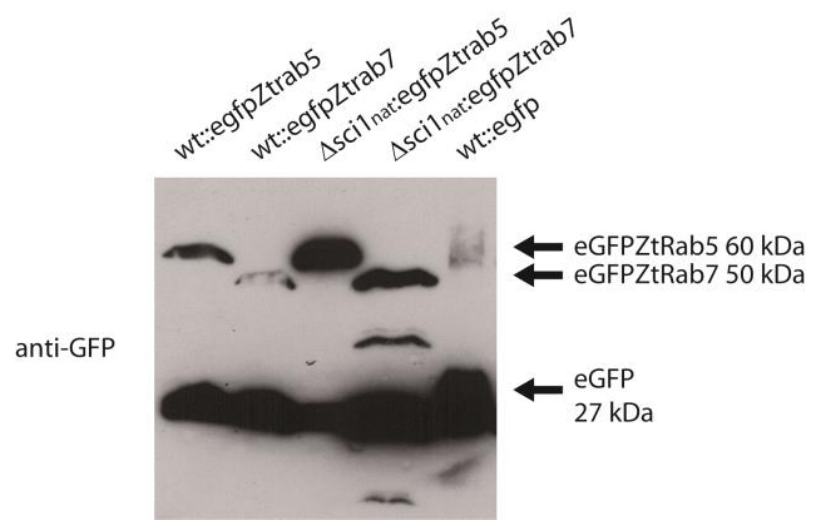

Figure S 16: Detection of eGFPZtRab5 and eGFPZtRab7 using anti-GFP in Western blot analysis

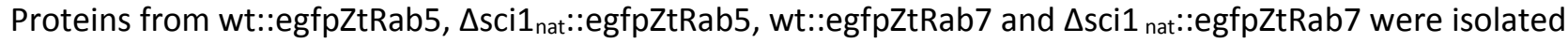
and Western blot was performed. Fusion proteins eGFPZtRab5 and eGFPZtRab7 were detected with antiGFP. The wt::egfp strain served as control. The apparent molecular weight of the presented fusion proteins is indicated in $\mathrm{kDa}$. 
A

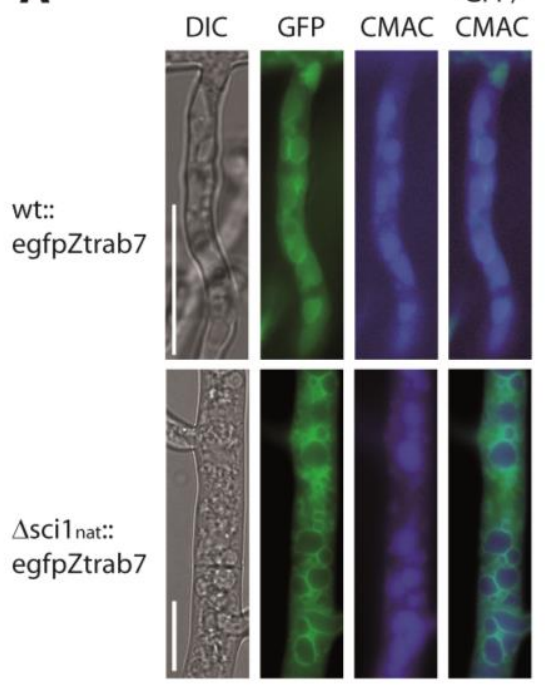

B

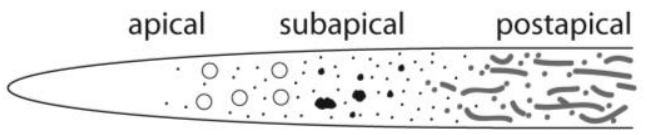

C

$\triangle$ Smmob3_gpdMOB3:Dsred

$\Delta$ sci1hyg:: sci1Dsred

$\Delta$ pro11:: hapro11egfp

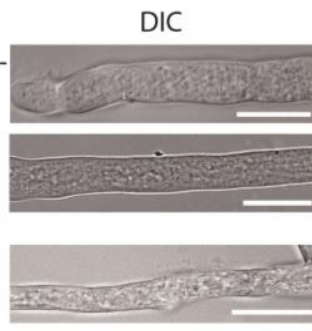

Figure S 17: Localization of the endosomal reporter eGFPZtRab7 and of SmMOB3DsRED, SCI1DsRED and HAPRO11eGFP fusion proteins in vegetative hyphae of $S$. macrospora

A: eGFPZtRab7 was observed in old hyphae of the $\Delta s c i 1_{\text {nat }}$ and wt background. Hyphae of wt::egfpZtRab7 and $\triangle$ sci1::egfpZtRab7 were stained with CMAC to visualize vacuoles in middle old to old hyphae. B: Representation of structures visualized by SmMOB3DsRED, SCI1DsRED and HAPRO11eGFP fusion proteins in the indicated strains. C: In each picture, from left to right, the apex, the subapical region and the postapical region are shown. Fluorescent signals in $\Delta$ Smmob3_gpd-MOB3:Dsred, $\Delta$ sci $1_{\text {hyg }}:$ :sci1Dsred and $\Delta$ pro11::hapro11egfp hyphae stained the nuclear envelope (white arrow), small or big punctiform structures and tubular structures. Scale bars: $20 \mu \mathrm{m}$.

Table S 1: Proteins enriched with HAPRO11 and with HAPRO11eGFP in pull-down experiments coupled to LC/MS

\begin{tabular}{|c|c|c|c|c|}
\hline Locus tag & Description & LC/MS1 & LC/MS2 & LC/MS3 \\
\hline SMAC_00646 & hypothetical protein & 10 & 4 & 39 \\
\hline SMAC_00877 & SmMOB3 & 3 & 45 & 30 \\
\hline SMAC_01171 & hypothetical protein & 7 & 2 & 10 \\
\hline SMAC_01542 & $\mathrm{N}$-acyltransferase & 12 & 10 & 20 \\
\hline SMAC_01602 & peptidase_C1B & 2 & 7 & 24 \\
\hline SMAC_01688 & RsmB, AdoMet-MTases & 11 & 2 & 27 \\
\hline SMAC_01724 & K homology RNA-binding domain, type I & 9 & 7 & 6 \\
\hline SMAC_02190 & pseudouridine synthase & 9 & 4 & 32 \\
\hline SMAC_02362 & thioredoxin_like & 12 & 4 & 25 \\
\hline
\end{tabular}


Supplement

\begin{tabular}{|c|c|c|c|c|}
\hline SMAC_02409 & putative nucleoporin protein/NIC 96 & 8 & 5 & 12 \\
\hline SMAC_02554 & rossmann-fold $N A D(P)(+)$-binding protein & 10 & 4 & 24 \\
\hline SMAC_02580 & PRO22 & 20 & 7 & 0 \\
\hline SMAC_02700 & tyrosine phosphatase family & 9 & 11 & 30 \\
\hline SMAC_02977 & oxysterol-binding protein & 2 & 7 & 4 \\
\hline SMAC_04212 & $\begin{array}{l}\text { putative kinesin group protein/ KIF4-like } \\
\text { subfamily }\end{array}$ & 8 & 7 & 3 \\
\hline SMAC_04377 & TreA, Neutral trehalase & 10 & 10 & 5 \\
\hline SMAC_04470 & VHS, ENTH and ANTH domain superfamily & 11 & 2 & 10 \\
\hline SMAC_04710 & serine/threonine kinase & 12 & 14 & 25 \\
\hline SMAC_04918 & Arf-like protein & 9 & 4 & 26 \\
\hline SMAC_05353 & serine/threonine protein kinase & 7 & 8 & 14 \\
\hline SMAC_05559 & $\mathrm{SCl} 1$ & 23 & 47 & 23 \\
\hline SMAC_06440 & ribosome-interacting GTPase 1 & 8 & 2 & 15 \\
\hline SMAC_07014 & $\begin{array}{l}\text { putative RheB-like protein/ Ras subfamily of } \\
\text { RAS small GTPases }\end{array}$ & 10 & 3 & 8 \\
\hline SMAC_07811 & Mo25-like & 16 & 4 & 26 \\
\hline SMAC_07827 & putative actin-2 protein/ centractin & 8 & 4 & 32 \\
\hline SMAC_08522 & heterokaryon incompatibility protein & 3 & 10 & 27 \\
\hline SMAC_08717 & $\mathrm{K}$ homology RNA-binding domain & 8 & 8 & 15 \\
\hline SMAC_08794 & PRO11 & 90 & 165 & 232 \\
\hline SMAC_08842 & glycyl-tRNA synthetase & 10 & 9 & 31 \\
\hline \multicolumn{5}{|c|}{$\begin{array}{l}\text { Pull-downs and LC/MS were preformed in three biological replicates (MS1-3). LC/MS data from HAPRO11 } \\
\text { and HAPRO11eGFP pull-downs were combined for analysis with the Proteome Discoverer }{ }^{\text {TM }} \text {. Criteria: at } \\
\text { least two peptides per protein, peptide mass confidence high, not enriched in the negative controls and sum } \\
\text { of spectral counts at least } 7 \text { in two of three LC/MS experiments. PRO11 indicated in bold was highly enriched } \\
\text { in all replicates. The sum of spectral counts in LC/MS1-3 are given in numbers. The locus tag and } \\
\text { corresponding description of protein (determined by the best BLASTP value) are given. }\end{array}$} \\
\hline
\end{tabular}


Table S 2: Proteins enriched with FLAGSmMOB3 and with SmMOB3eGFP in pull-down experiments coupled to LC/MS

\begin{tabular}{lllll} 
Locus tag & Description & \multicolumn{2}{l}{ LC/MS1 } & \multicolumn{2}{l}{ LC/MS2 } & LC/MS3 \\
\hline SMAC_00877 & SmMOB3 & 80 & 66 & 122 \\
\hline SMAC_02409 & putative nucleoporin protein/NIC 96 & 12 & 6 & 8 \\
\hline SMAC_04517 & hypothetical protein & 9 & 6 & 11 \\
\hline SMAC_05008 & putative myosin-5 protein & 11 & 6 & 9 \\
\hline SMAC_08794 & PRO11 & 80 & 88 & 124 \\
\hline SMAC_09495 & AP-2 complex subunit alpha & 9 & 6 & 11
\end{tabular}

Pull-downs and LC/MS were preformed in three biological replicates (MS1-3). LC/MS data from FLAGSmMOB3 and SmMOB3eGFP pull-downs were combined for analysis with the Proteome Discoverer ${ }^{\mathrm{TM}}$. Criteria: at least two peptides per protein, peptide mass confidence high, not enriched in the negative controls and sum of spectral counts at least 7 in two of three LC/MS experiments. SmMOB3 indicated in bold was highly enriched in all replicates. The sum of spectral counts in LC/MS1-3 are given in numbers. The locus tag and corresponding description of protein (determined by the best BLASTP value) are given.

Table S 3: Proteins enriched with HAPRO11/HAPRO11eGFP and FLAGSmMOB3/SmMOB3eGFP in pulldown experiments coupled to LC/MS

\begin{tabular}{|c|c|c|c|c|c|c|c|}
\hline Locus tag & Description & $\begin{array}{c}\text { LC/MS } \\
\quad 1\end{array}$ & $\begin{array}{c}\mathrm{LC} / \mathrm{MS} \\
2\end{array}$ & $\begin{array}{l}\text { LC/MS } \\
\quad 3\end{array}$ & $\begin{array}{c}\text { LC/MS } \\
1\end{array}$ & $\begin{array}{l}\mathrm{LC} / \mathrm{MS} \\
2\end{array}$ & $\begin{array}{c}\text { LC/MS } \\
3\end{array}$ \\
\hline & & \multicolumn{3}{|c|}{$\begin{array}{l}\text { HAPRO11/ } \\
\text { HAPRO11GFP }\end{array}$} & \multicolumn{3}{|c|}{$\begin{array}{l}\text { FLAGSmMOB3/ } \\
\text { SmMOB3eGFP }\end{array}$} \\
\hline SMAC_00134 & $\begin{array}{l}\text { serine/threonine-protein } \\
\text { phosphatase } \\
\text { PP1/Calcineurin like } \\
\text { phosphoesterase }\end{array}$ & 8 & & 30 & 7 & & 18 \\
\hline SMAC_00877 & SmMOB3 & 3 & 45 & 30 & 80 & 66 & 122 \\
\hline SMAC_01282 & $\begin{array}{l}\text { carbamoyl-phosphate } \\
\text { synthase subunit L }\end{array}$ & & 14 & 40 & & 5 & 15 \\
\hline SMAC_02409 & $\begin{array}{l}\text { putative nucleoporin } \\
\text { protein/NIC } 96\end{array}$ & 8 & 5 & 12 & 12 & 6 & 8 \\
\hline SMAC_02580 & PRO22 & 20 & 7 & & & 7 & 7 \\
\hline SMAC_05008 & putative myosin-5 protein & 11 & & 16 & 11 & 6 & 9 \\
\hline SMAC_05559 & SCl1 & 23 & 47 & 23 & 6 & & 13 \\
\hline
\end{tabular}


\begin{tabular}{|l|l|l|l|l|l|l|l|}
\hline SMAC_08794 & PRO11 & 90 & 165 & 232 & 80 & 88 & 124
\end{tabular}

Pull-downs and LC/MS were preformed in three biological replicates (MS1-3). LC/MS data from HAPRO11/HAPRO11eGFP and FLAGSmMOB3/SmMOB3eGFP pull-downs were combined for analysis with the Proteome Discoverer ${ }^{\mathrm{TM}}$. Criteria: at least two peptides per protein, peptide mass confidence high, not enriched in the negative controls, sum of spectral counts at least 5 in two of three MS experiments of PRO11 and SmMOB3 pull-downs, respectively. The sum of spectral counts in LC/MS1-3 are given in numbers. PRO11 or SmMOB3 indicated in bold were highly enriched in the corresponding replicates. The locus tag and corresponding description of protein (determined by the best BLASTP value) are given.

Table S 4: Proteins enriched with SCl1eGFP in pull-down experiments coupled to LC/MS

\begin{tabular}{|c|c|c|c|c|}
\hline Locus tag & Description & LC/MS1 & LC/MS2 & LC/MS3 \\
\hline SMAC_00550 & putative transcription factor & 7 & 10 & 2 \\
\hline SMAC_00761 & dynein heavy chain & 15 & 14 & 25 \\
\hline SMAC_00774 & nucleoporin POM33 & 15 & 20 & 3 \\
\hline SMAC_01320 & $\begin{array}{l}\text { nascent polypeptide-associated complex } \\
\text { (NACA) subunit alpha }\end{array}$ & 8 & 13 & 12 \\
\hline SMAC_01363 & $\begin{array}{l}\text { casein kinase, serine/threonine protein kinase } \\
\text { HRR25 involved in vesicular traffic }\end{array}$ & 13 & 15 & 9 \\
\hline SMAC_01428 & glycogen debranching enzyme & 5 & 7 & 8 \\
\hline SMAC_02146 & $\begin{array}{l}\text { protein kinase YAK-1, Serine-threonine protein } \\
\text { kinase }\end{array}$ & 9 & 11 & 3 \\
\hline SMAC_03109 & chitin synthase & 11 & 26 & 12 \\
\hline SMAC_03125 & $\begin{array}{l}\text { Sec14 family involved in transport into and } \\
\text { out Golgi, secretion from the trans-Golgi }\end{array}$ & 9 & 6 & 4 \\
\hline SMAC_03130 & polyketide synthase & 6 & 10 & 7 \\
\hline SMAC_03452 & phosphoglycerate dehydrogenase & 12 & 15 & 2 \\
\hline SMAC_03809 & cytohesin-2/Sec7 & 8 & 15 & 4 \\
\hline SMAC_04234 & $\begin{array}{l}\text { putative STT4 protein, normal vacuole } \\
\text { morphology, cell wall integrity, and actin } \\
\text { cytoskeleton organization }\end{array}$ & 9 & 10 & 12 \\
\hline SMAC_05559 & SCl1 & 371 & 481 & 641 \\
\hline SMAC_05628 & $\begin{array}{l}\text { Alcohol dehydrogenase zinc-binding domain } \\
\text { protein }\end{array}$ & 9 & 25 & 6 \\
\hline SMAC_05656 & $\begin{array}{l}\text { NmrA family protein, controlling nitrogen } \\
\text { metabolite repression }\end{array}$ & 13 & 28 & 6 \\
\hline SMAC_06043 & glycosyltransferase family 20 protein & 8 & 5 & 10 \\
\hline SMAC_06237 & Iki3 family protein & 8 & 13 & 9 \\
\hline
\end{tabular}


Supplement

\begin{tabular}{|l|l|l|l|l}
\hline SMAC_06397 & putative RasGAP & 14 & 8 & 2 \\
\hline SMAC_07234 & aminotransferase & 15 & 16 & 4 \\
\hline SMAC_08299 & ARM repeat-containing protein, Vac14, & 16 & 31 & 2 \\
\hline SMAC_08770 & non-anchored cell wall protein 1 & 15 & 16 & 6 \\
\hline SMAC_08794 & PRO11 & 73 & 81 & 110
\end{tabular}

Pull-downs and LC/MSs were preformed in three biological replicates (MS1-3). LC/MS data from SCI1eGFP pull-downs were combined for analysis with the Proteome Discoverer ${ }^{\mathrm{TM}}$. Criteria: at least two peptides per protein, peptide mass confidence high, not enriched in the negative controls and sum of spectral counts at least 7 in two of three LC/MS experiments. SCl1eGFP indicated in bold was highly enriched in all replicates. The sum of spectral counts in LC/MS1-3 are given in numbers. The locus tag and corresponding description of protein (determined by the best BLASTP value) are given. Proteins identified and defined as significant with MaxQuant/Perseus are marked in orange.

\section{Video 1: Nuclei in young hyphae of wt::h2btdTomato}

Nuclei were visualized by histone $2 \mathrm{~B}$ fused to TdTomato and move freely within the hyphae of wt::h2btdTomato. Recording: 46 stacks, exposure time: $100 \mathrm{~ms} / 2 \mathrm{sec}$ for $1.5 \mathrm{~min}$, video length: $1.077 \mathrm{~min}$, DICV1: Hyphae in visualized by differential interference contrast (DIC). Scale bar: $20 \mu \mathrm{m}$

\section{Video 2: Nuclei in young hyphae of $\Delta s c i 1_{\text {hyg }}:$ h2 2 btdTomato}

Nuclei were visualized by histone $2 \mathrm{~B}$ fused to TdTomato and move freely within the hyphae of $\Delta$ sci $1_{\text {hyg }}:$ h2btdTomato. Recording: 61 stacks, exposure time: $500 \mathrm{~ms} / 1 \mathrm{sec}$ for $1 \mathrm{~min}$, video length: 1.508 min, DICV2: Hyphae in visualized by differential interference contrast (DIC). Scale bar: $20 \mu \mathrm{m}$

\section{Video 3: Mitochondria in young hyphae of wt::mitoDsred}

The reporter protein CAS2(AA1-60) fused to DsRED visualized mitochondria in hyphae of wt::mitoDsred strain. DICV3: Hyphae in visualized by differential interference contrast (DIC). Recording: 31 stacks, exposure time: $500 \mathrm{~ms} / 2 \mathrm{sec}$ for $1 \mathrm{~min}$, video length: $1.258 \mathrm{~min}$, Scale bar: $20 \mu \mathrm{m}$

Video 4: Mitochondria in hyphae of $\Delta$ sci1 $1_{\text {hyg: }}$ mitoDsred

The reporter protein CAS2(AA1-60) fused to DsRED visualized mitochondria in hyphae of $\Delta$ sci1::mitoDsred. Recording: 91 stacks, exposure time: $100 \mathrm{~ms} / 2$ sec for $3 \mathrm{~min}$, video length: $3.152 \mathrm{~min}$, DICV4: Hyphae in visualized by differential interference contrast (DIC). Scale bar: $20 \mu \mathrm{m}$

\section{Video 5: Actin polymerization in young hyphae of wt::lifeact-tagrfp}

The wt strain was transformed with the plasmid pAL-4Lifeact and actin polymerization in young hyphae was visualized. Lifeact-TagRFP visualized actin, distributed in a defined region along the cell wall. Than, actin polymerizes in a hill like structure, which most likely represents the contraction of the actin ring preceding septum formation. Recording: 25 stacks, exposure time: $500 \mathrm{~ms} / 1 \mathrm{sec}$ for $0.8 \mathrm{~min}$, video length: $1.008 \mathrm{~min}$, DICV5: Hyphae in visualized by differential interference contrast (DIC). Scale bar: $20 \mu \mathrm{m}$ 
Video 6: Actin polymerization in hyphae of $\Delta s c i 1_{\text {hyg }}:$ lifeact-tagrfp

The $\Delta$ sci1 strain was transformed with the plasmid pAL-4Lifeact to visualize actin polymerization in young hyphae. Although, the Lifeact-TagRFP signal was very weak in $\Delta$ sci1::lifeact-tagrfp compared to the wt::lifeact-tagrfp actin polymerization was visualized. Actin was distributed in a defined region along the cell wall, than, formed a hill like structure, which most likely represents the contraction of the actin ring preceding septum formation. DICV6: Hyphae in visualized by differential interference contrast (DIC). Recording: 31 stacks, exposure time: $500 \mathrm{~ms} / 1 \mathrm{sec}$ for $1 \mathrm{~min}$, video length: $1.258 \mathrm{~min}$, Scale bar: $20 \mu \mathrm{m}$

Video 7: Structures visualized by early endosome marker eGFPZtRab5 in young hyphae of wt::egfpZtrab5 The plasmid pHEGFPRAB5 was transformed into the wt strain. The EE marker eGFPZtRab5 visualized EE, continuously changing punctiform structures and tubular/vacuolar structures in young hyphae. Recording: 121 stacks, exposure time: 100 ms/1 sec for 2 min, video length: 2.202 min, DICV7: Hyphae in visualized by differential interference contrast (DIC). Scale bar: $20 \mu \mathrm{m}$

Video 8: Structures visualized by EE marker eGFPZtRab5 in young hyphae of $\Delta$ sci1 nat::egfpZtrab5 The plasmid pHEGFPRAB5 was transformed into the $\triangle$ sci1 strain. The EE marker eGFPZtRab5 visualized EE, big punctiform structures incorporating vesicles. Recording: 121 stacks, exposure time: $200 \mathrm{~ms} / 1 \mathrm{sec}$ for 2 min, video length: 2.403 min, DICV8: Hyphae in visualized by differential interference contrast (DIC). Scale bar: $20 \mu \mathrm{m}$

Video 9: The EE marker eGFPZtRab5 in young hyphae of $\Delta$ sci1 $1_{\text {nat }}:$ :egfpZtrab5 visualized "cloudy" structures In the post-apical region of young hyphae of $\Delta$ sci1::egfpZtrab5 sometimes, big punctiform structures dissolved into "clouds" around dark regions most likely representing nuclei and reformed again. Recording: 121 stacks, exposure time: $100 \mathrm{~ms} / 1 \mathrm{sec}$ for $2 \mathrm{~min}$, video length: $2.202 \mathrm{~min}$, DICV9: Hyphae in visualized by differential interference contrast (DIC). Scale bar: $20 \mu \mathrm{m}$

Video 10: Structures visualized by late endosome marker eGFPZtRab7 in young hyphae of wt::egfpZtrab7 The plasmid pHEGFPRAB7 was transformed into the wt strain. The LE marker eGFPZtRab7 visualized LEs fusing and splitting again to and from tubular structures in young hyphae. DICV10: Hyphae in visualized by differential interference contrast (DIC). Recording: 121 stacks, exposure time: $300 \mathrm{~ms} / 1 \mathrm{sec}$ for $2 \mathrm{~min}$, video length: $2.605 \mathrm{~min}$, Scale bar: $20 \mu \mathrm{m}$

Video 11: Structures visualized by the LE marker eGFPZtRab7 in young hyphae of $\Delta$ sci1 $1_{\text {nat }}:$ :egfpZtrab7 The plasmid pHEGFPRAB7 was transformed into the $\Delta$ sci1 strain. The LE marker eGFPZtRab7 visualized vesicles and vacuolar structures in young hyphae of $\Delta$ sci1::egfpZtrab7, which were scattered compared to the wt and did not form a long tubular network. Recording: 121 stacks, exposure time: $300 \mathrm{~ms} / 1 \mathrm{sec}$ for 2 min, video length: 2.605 min, DICV11: Hyphae in visualized by differential interference contrast (DIC). Scale bar: $20 \mu \mathrm{m}$ 


\section{Danksagung}

\section{Danksagung}

An erster Stelle möchte ich mich herzlich bei meiner Doktormutter, Prof. Dr. Stefanie Pöggeler, für die Möglichkeit in ihrer Arbeitsgruppe zu promovieren, bedanken. Ich danke ihr insbesondere für ihr fortwährendes Interesse an dieser Forschungsarbeit, die hilfreichen Anregungen, die wissenschaftlichen und persönlichen Diskussionen, sowie das mir entgegengebrachte Vertrauen.

Außerdem bedanke ich mich bei den Mitgliedern meines „Thesis Committees“, Prof. Dr. Gerhard Braus und Prof. Dr. Henning Urlaub für die wertvollen Hinweise und Anmerkungen, die maßgeblich in diese Arbeit miteingeflossen sind.

Im Zuge dessen möchte ich mich auch bei Dr. Oliver Valerius für die Zusammenarbeit bedanken, die dazu beigetragen hat, den Fortgang dieses Projekt zu ermöglichen.

Ich möchte mich auch bei Dr. Britta Herzog und Dr. Steffen Nordzieke für die Durchsicht dieser Dissertationsarbeit, sowie Dr. Steffen Nordzieke für die ein oder anderen humorvollen Bemerkungen und für die „Last-Minute-Bilder“ bedanken. Des Weiteren gilt mein Dank auch Dr. Antonia Werner und Dr. Daniela Nordzieke für die kollegiale Zusammenarbeit, Unterstützung und den Austausch über wissenschaftliche sowie private Themen.

Gertrud Stahlhut danke ich für ihre Mithilfe an den Experimenten die sich in dieser Arbeit wiederfinden und vor allem für ihre immer ehrliche, offene und hilfsbereite Art. Mein Dank gilt ebenso Silvia Carrillo-Castellón, die sich um die Versorgung mit Arbeitsmaterialien kümmert. Auch danke ich Fereshteh Sadeqi, die während ihrer Bachelor Arbeit an diesem Projekt mitgearbeitet hat und Dr. Blaga Popova für die Hilfe in „Hefe-Fragen“.

Meinen Eltern und meiner Schwester danke ich besonders für die Motivation und die Unterstützung in allen Lebenslagen. Daneben gilt mein Dank meinen Schwiegereltern und meinem Schwager für den Rückhalt. Zwei besonderen Menschen möchte ich noch danken, meinem Ehemann für seine grenzenlose Geduld und Unterstützung sowie meinem Sohn. Ihr erfüllt mein Leben jeden Tag mit Sinn und Freude. 


\section{Curriculum vitae}

Eva Johanna Reschka

Georg-August University Göttingen

Institute of Microbiology and Genetics

Department of Genetics of Eukaryotic Microorganisms

Grisebachstraße 8

Göttingen, Germany

E-mail: ereschk@gwdg.de

Date of birth: July $17^{\text {th }} 1984$

Place of birth: Tarnowitz (Poland)

Nationality: German

\section{Academic Education:}

Since 03/2013 PhD student in the GGNB program "Microbiology and Biochemistry", Department of Genetics of Eukaryotic Microorganisms, Georg-August University Göttingen, Germany

Title of the Thesis: Functional analysis of STRIPAK complex components in the filamentous ascomycete Sordaria macrospora

Thesis Committee: Prof. Dr. Stefanie Pöggeler, Prof. Dr. Gerhard Braus, Prof. Dr. Henning Urlaub

12/ 2012- 02/2013 Research assistant, Department of Genetics of Eukaryotic Microorganisms, Georg-August University Göttingen, Germany

10/2010-09/ 2012 Master of Science, M.Sc Microbiology and Biochemistry

Department of Genetics of Eukaryotic Microorganisms, Georg-August University Göttingen, Germany

Title of the Master thesis: Co-IP of STRIPAK complex proteins in Sordaria macrospora and identification of new SmPP2AA and PRO11 interaction partners

09/2007-07/2010 Bachelor of Science, B.Sc Applied Biology, Hochschule Bonn-Rhein-Sieg University of Applied Sciences, Germany

„Double Degree” Bioscience with Biomedical Science, Robert Gordon University Aberdeen, UK

Title of the Bachelor thesis: Sex identification of mammals by PCR amplification of $\mathrm{Zfx} / \mathrm{Zfy}$ intronic regions

\section{School Education:}

09/2001 - 06/ 2004 Konrad-Adenauer-Gymnasium, Bad Godesberg (Abitur) 
Talks:

$10 / 2015$

Minisymposium, Department for General and Molecular Botany,

Prof. Dr. Ulrich Kück, Ruhr-University Bochum, Germany

"SCI1, a new STRIPAK interaction partner in Sordaria macrospora"

$10 / 2015$

Summerschool 2015, Proteomics and Mass Spectrometry, Department of Genetics of Eukaryotic Microorganisms, Georg-August University Göttingen, Germany

"Analysis of LC/MS data identified several STRIPAK complex components in Sordaria macrospora"

$10 / 2014$

Retreat of the GGNB program "Microbiology and Biochemistry"

"Identification and characterization of new STRIPAK complex components in Sordaria macrospora"

Posters:

$12 / 2016$

$10 / 2015$

4th GGNB Science Day at the Georg-August University Göttingen, Germany "A new STRIPAK interaction partner in Sordaria macrospora"

VAAM 2014 Berlin, Germany "A new STRIPAK interaction partner in Sordaria macrospora"

$03 / 2015$

28th Fungal Genetics Conference in Asilomar, California, USA

"Analysis of SCI1, a potential new interaction partner of the STRIPAK complex"

$10 / 2014$

VAAM 2014 Dresden, Germany "Analysis of SCI1, a potential interaction partner of the STRIPAK complex"

\section{Publications:}

Reschka, E.J., Nordzieke, S., Valerius, O., Braus, G., Pöggeler, S.

A novel STRIPAK complex component mediates hyphal fusion and fruiting-body development in filamentous fungi (in prep.)

Frey, S., Reschka, E.J. \& Pöggeler, S., (2015) Germinal center kinases SmKIN3 and SmKIN24 are associated with the Sordaria macrospora striatin-interacting phosphatase and kinase (STRIPAK) complex. PLOS One 10: e0139163. 